\title{
Expanding Ligand Space: Preparation, Characterization and Synthetic Applications of Air-Stable, Odorless Di-tert-alkylphosphine Surrogates
}

\author{
Thomas Barber, ${ }^{\dagger, \ddagger}$ Stephen P. Argent, ${ }^{\dagger}$ and Liam T. Ball ${ }^{\dagger, \ddagger}, *$ \\ ${ }^{+}$School of Chemistry, University of Nottingham, University Park, Nottingham, NG7 2RD, U.K. \\ ‡ GSK Carbon Neutral Laboratories for Sustainable Chemistry, University of Nottingham, \\ Jubilee Campus, Triumph Road, Nottingham, NG7 2TU, U.K. \\ *Correspondence to: liam.ball@nottingham.ac.uk
}




\section{Contents}

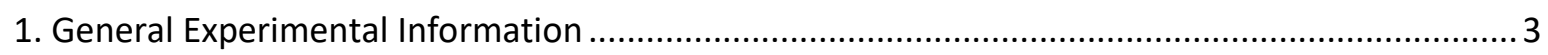

2. Synthesis and Characterisation of tert-Alkyl Esters ............................................................. 5

3. Determination of $\mathrm{Zn}_{3} \mathrm{P}_{2}$ Purity and Protonolysis Rate (Manuscript Scheme 2B) ..........................26

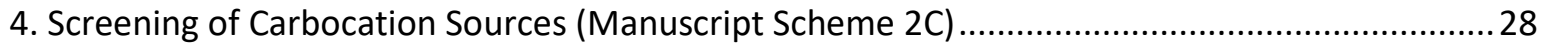

5. Synthesis of Di-tert-alkylphosphonium Salts 1 (Manuscript Scheme 3) ...................................... 29

6. Alkylation of $\mathrm{PH}_{3}$ with tert-Butyl Acetate (Manuscript Scheme 4A) .......................................... 45

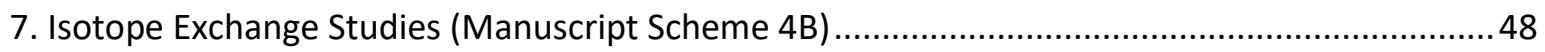

8. Derivatisation of Di-(3-ethyl-3-pentyl)phosphonium Triflate 1c (Manuscript Scheme 5) .............55

9. Synthesis and Characterisation of Di-tert-alkylbiarylphosphines 3 (Manuscript Scheme 6) ........59

10. Synthesis of Di-tert-alkylbiarylphosphine Selenides 4 (Manuscript Scheme 6) .........................69

11. Synthesis and Characterisation of Di-tert-alkylbiarylphosphine Gold(I) Chloride Complexes 5

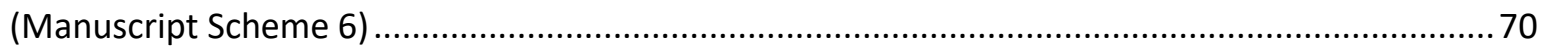

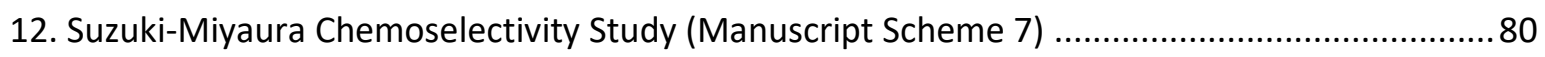

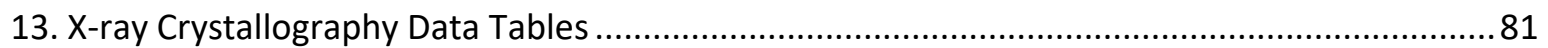

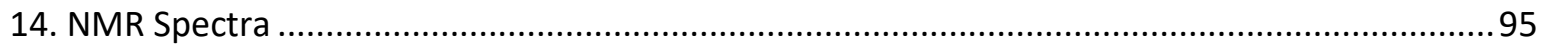

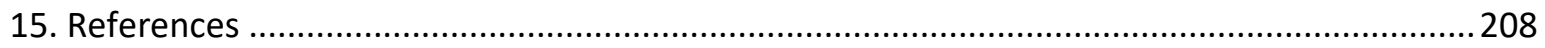




\section{General Experimental Information}

CAUTION: phosphine gas is highly toxic and is spontaneously flammable in air. Procedures that use phosphine gas should only be performed following appropriate training after consultation with local safety advisors, and after completing a thorough risk assessment. All CO-ware apparatus should be inspected for scratches or imperfections prior to use; new CO-ware septa should be used for each reaction, and the manufacturer's guidelines should be followed (no more than 2 injections per septum, using a needle with a diameter of $21 \mathrm{~g}(0.08 \mathrm{~mm})$ or less). CO-ware is recommended for routine use at pressures up to $46 \mathrm{psi}$, and not above $60 \mathrm{psi}$; this should be considered when changing reaction scales from those presented in this Supporting Information.

Reagents were purchased from commercial suppliers and used as provided. Me $\mathrm{MSiOTf}_{3}$ and methylmagnesium bromide (3.0 $\mathrm{M}$ in $\mathrm{Et}_{2} \mathrm{O}$ ) were stored under anhydrous dinitrogen in sample flasks with J. Young valves. Anhydrous tetrahydrofuran, toluene, dichloromethane and diethyl ether were obtained from in-house solvent purification systems (Inert ${ }^{\circ}$ ProSolv; dried by passage through activated alumina columns under pressure of $\mathrm{Ar}$ ). Anhydrous $\mathrm{CCl}_{4}$ and $\mathrm{DBU}$ were obtained by distillation from $\mathrm{CaH}_{2}$ under an atmosphere of anhydrous dinitrogen. Deuterated solvents were used as supplied. Reactions requiring inert conditions were conducted in flame-dried glassware (apart from NMR tubes and CO-ware two-chamber reactor) under an atmosphere of anhydrous dinitrogen using standard Schlenk techniques.

NMR spectra were recorded at $298 \mathrm{~K}$ on Bruker Avance-III spectrometers operating at nominal ${ }^{1} \mathrm{H}$ frequencies of 400 and $500 \mathrm{MHz}$. Chemical shifts ( $\delta$ ) are given in ppm, and are referenced to residual solvent peaks for ${ }^{1} \mathrm{H}$ and ${ }^{13} \mathrm{C}\left\{{ }^{1} \mathrm{H}\right\} . \mathrm{CDCl}_{3}$ (7.26 ppm; $77.16 \mathrm{ppm}$ ), $\mathrm{C}_{6} \mathrm{D}_{6}$ (7.16 ppm; $128.06 \mathrm{ppm}$ ), $\mathrm{CD}_{3} \mathrm{CN}$ (1.94 ppm; 1.32 and 118.26 ppm). External frequency standards are used for ${ }^{11} \mathrm{~B}\left(\mathrm{BF}_{3} . \mathrm{OEt}_{2} ; 0.00 \mathrm{ppm}\right)$, ${ }^{19} \mathrm{~F}\left(\mathrm{CCl}_{3} \mathrm{~F} ; 0.00 \mathrm{ppm}\right)$ and ${ }^{31} \mathrm{P}\left(85 \% \mathrm{H}_{3} \mathrm{PO}_{4(\mathrm{aq})} ; 0.00 \mathrm{ppm}\right)$. Signals are reported as singlet (s), doublet (d), triplet $(t)$, quartet $(q)$, pentet $(p)$, heptet $(h)$, multiplet $(m)$, broad (br.), apparent (app.) or combinations thereof. Higher multiplicities are expected in several compounds (vide infra), but are reported as multiplets since it was not possible to observe every peak. Due to broadness of peaks, one-bond phosphorus-proton coupling constants measured from the ${ }^{31} \mathrm{P}$ NMR spectra are in many cases not matching the same constant when measured from the ${ }^{1} \mathrm{H}$ spectrum, though the differences are never $>0.5 \%)$. A 30 second relaxation delay time $\left(D_{1}\right)$ was used for quantitative ${ }^{19} \mathrm{~F}$ and ${ }^{31} \mathrm{P}$ NMR spectroscopy.

High-resolution mass spectrometry (HRMS) was performed using a Bruker MicroTOF spectrometer, with an electrospray ionisation (ESI) ion source. Infrared spectra (IR) were recorded on a Bruker Alpha 
platinum-ATR with diamond window. Melting points were measured on a Gallenkamp melting point apparatus and are uncorrected. 


\section{Synthesis and Characterisation of tert-Alkyl Esters}

General procedure for the synthesis of tert-alkyl esters (GP1)

$\mathrm{R}^{-\mathrm{OH}} \underset{\text { pyridine, } \Delta}{\stackrel{\mathrm{Ac}_{2} \mathrm{O}, \mathrm{DMAP}}{\longrightarrow}} \mathrm{R}^{-} \prod_{\mathrm{O}}^{\mathrm{O}}$

A mixture of alcohol (1.0 equiv.), acetic anhydride (1.1-1.5 equiv.) and 4-dimethylaminopyridine (DMAP, 0-0.1 equiv.) in pyridine was heated to $100{ }^{\circ} \mathrm{C}$ in a sealed microwave tube overnight. The resulting mixture was diluted with saturated aqueous $\mathrm{NaHCO}_{3}$ solution and extracted with $\mathrm{Et}_{2} \mathrm{O}(3 \times)$. The combined organic portions were washed with saturated aqueous $\mathrm{CuSO}_{4}$ solution $(3 \times)$, dried $\left(\mathrm{MgSO}_{4}\right)$, filtered and concentrated in vacuo. Note: care should be exercised to minimise the loss of the more volatile, low molecular weight esters. Crude products were purified by kugelrohr distillation at the temperatures and pressures stated for individual compounds. 


\section{3-Methyl-3-pentyl acetate}

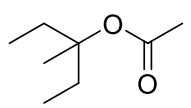

GP1 was performed using 3-methyl-3-pentanol $(6.20 \mathrm{~mL}, 50.0 \mathrm{mmol})$, acetic anhydride $(7.10 \mathrm{~mL}, 75.0$ mmol), pyridine $(6.0 \mathrm{~mL})$ and DMAP $(0.611 \mathrm{~g}, 5.00 \mathrm{mmol})$. After kugelrohr distillation $\left(40-45{ }^{\circ} \mathrm{C}, 10\right.$ mbar), 3-methy-3-pentyl acetate $(5.27 \mathrm{~g}, 36.5 \mathrm{mmol}, 73 \%)$ was obtained as a colourless liquid.

${ }^{1} \mathrm{H}$ NMR (500 MHz, CDCl $): \delta 1.98(\mathrm{~s}, 3 \mathrm{H}), 1.90-1.82(\mathrm{~m}, 2 \mathrm{H}), 1.77-1.69(\mathrm{~m}, 2 \mathrm{H}), 1.36(\mathrm{~s}, 3 \mathrm{H}), 0.85$ (app. $\mathrm{t}, J=7.5 \mathrm{~Hz}, 6 \mathrm{H})$.

${ }^{13} \mathrm{C}\left\{{ }^{1} \mathrm{H}\right\}$ NMR (126 MHz, $\left.\mathrm{CDCl}_{3}\right): \delta$ 170.4, 85.4, 30.4, 22.8, 22.3, 8.0.

$v_{\max }($ neat $) / \mathrm{cm}^{-1}: 2974,2942,2884,1730,1461,1367,1246,1157,1136,1017,940,848,610$.

HRMS: calcd. for $\mathrm{C}_{8} \mathrm{H}_{16} \mathrm{O}_{2}+\mathrm{Na}^{+}$: $167.1043[\mathrm{M}+\mathrm{Na}]^{+}$; found $\left.(\mathrm{ESI})^{+}\right): 167.1034$. 


\section{3-Ethyl-3-pentyl acetate}

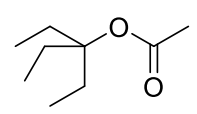

GP1 was performed using 3-ethyl-3-pentanol $(6.87 \mathrm{~mL}, 50.0 \mathrm{mmol})$, acetic anhydride $(7.10 \mathrm{ml}, 75.0$ $\mathrm{mmol})$, pyridine $(6.0 \mathrm{~mL})$, and DMAP $(0.611 \mathrm{~g}, 5.00 \mathrm{mmol})$. After kugelrohr distillation $\left(45^{\circ} \mathrm{C}, 1.0 \mathrm{mbar}\right)$ 3-ethyl-3-pentyl acetate $(1.36 \mathrm{~g}, 8.59 \mathrm{mmol}, 43 \%)$ was obtained as a colourless liquid.

${ }^{1} \mathrm{H}$ NMR (500 MHz, CDCl $): \delta 1.99(\mathrm{~s}, 3 \mathrm{H}), 1.81(\mathrm{q}, J=7.5 \mathrm{~Hz}, 6 \mathrm{H}), 0.81(\mathrm{t}, J=7.5 \mathrm{~Hz}, 9 \mathrm{H})$.

${ }^{13} \mathrm{C}\left\{{ }^{1} \mathrm{H}\right\}$ NMR (126 MHz, $\left.\mathrm{CDCl}_{3}\right): \delta 170.4,88.3,26.9,27.4,7.8$.

$V_{\max }($ neat $) / \mathrm{cm}^{-1}: 2971,2944,2884,1727,1458,1366,1244,1205,1138,1040,1014,952,924,864$, 610.

HRMS: Neither the parent ion nor any obvious fragments could be observed by ESI-MS. 


\section{2,3-Dimethyl-2-butyl acetate}<smiles>CC(=O)OC(C)(C)C(C)C</smiles>

GP1 was performed using 2,3-dimethyl-2-butanol $(3.72 \mathrm{~mL}, 30.0 \mathrm{mmol})$, acetic anhydride $(4.25 \mathrm{~mL}$, $45.0 \mathrm{mmol})$, pyridine $(5.0 \mathrm{~mL})$ and DMAP $(0.366 \mathrm{~g}, 3.00 \mathrm{mmol})$. After kugelrohr distillation (r.t., 1.0 mbar), 2,3-dimethyl-2-butyl acetate $(2.44 \mathrm{~g}, 16.9 \mathrm{mmol}, 56 \%)$ was obtained as a colourless liquid.

${ }^{1} \mathrm{H}$ NMR $\left(500 \mathrm{MHz}, \mathrm{CDCl}_{3}\right): \delta 2.20(\mathrm{~h}, J=6.9 \mathrm{~Hz}, 1 \mathrm{H}), 1.96(\mathrm{~s}, 3 \mathrm{H}), 1.38(\mathrm{~s}, 6 \mathrm{H}), 0.88(\mathrm{~d}, J=6.9 \mathrm{~Hz}, 6 \mathrm{H})$. ${ }^{13} \mathrm{C}\left\{{ }^{1} \mathrm{H}\right\}$ NMR (126 MHz, $\left.\mathrm{CDCl}_{3}\right): \delta 170.7,85.5,36.2,22.9,22.6,17.4$.

$v_{\max }($ neat $) / \mathrm{cm}^{-1}: 2972,1728,1464,1368,1250,1230,1140,1097,1070,1017,939,897,820,609$. HRMS: Neither the parent ion nor any obvious fragments could be observed by ESI-MS. 


\section{2,3,3-Trimethyl-2-butanol ${ }^{1}$}<smiles>CCOC(=O)C(C)(C)C(C)(C)O</smiles>

To a solution of pinacolone $(6.25 \mathrm{~mL}, 50.0 \mathrm{mmol})$ in anhydrous $\mathrm{Et}_{2} \mathrm{O}(20 \mathrm{~mL})$ under nitrogen was added methylmagnesium bromide ( $3.0 \mathrm{M}$ in $\mathrm{Et}_{2} \mathrm{O} ; 20.0 \mathrm{~mL}, 60.0 \mathrm{mmol}$ ). The mixture was stirred at r.t. for 16 $h$ before being quenched with a saturated aqueous solution of $\mathrm{NH}_{4} \mathrm{Cl}$. The resulting biphasic mixture was separated, and the aqueous portion was extracted with $\mathrm{Et}_{2} \mathrm{O}(3 \times)$. The organic layers were combined, dried $\left(\mathrm{MgSO}_{4}\right)$, filtered and the solvent was removed in vacuo to give 2,3,3-trimethyl-2butanol (5.76 g, $49.6 \mathrm{mmol}, 99 \%)$ as a colourless liquid. This material was used without further purification.

Characterisation data were consistent with literature values: ${ }^{1} \mathrm{H}^{1}$ and ${ }^{13} \mathrm{C}\left\{{ }^{1} \mathrm{H}\right\}^{1} \mathrm{NMR}$.

${ }^{1} \mathrm{H}$ NMR (500 MHz, $\left.\mathrm{CDCl}_{3}\right): \delta 1.28(\mathrm{~s}, 1 \mathrm{H}), 1.18(\mathrm{~s}, 6 \mathrm{H}), 0.94(\mathrm{~s}, 9 \mathrm{H})$.

${ }^{13} \mathrm{C}\left\{{ }^{1} \mathrm{H}\right\}$ NMR (126 MHz, $\left.\mathrm{CDCl}_{3}\right): \delta$ 75.1, 37.5, 25.5, 25.4.

$V_{\max }($ neat $) / \mathrm{cm}^{-1}:$ 3466, 2972, 2914, 2876, 1467, 1368, 1152, 1117, 944, 909, 879, 811, 734, 514.

HRMS: Neither the parent ion nor any obvious fragments could be observed by ESI-MS. 


\section{2,3,3-Trimethyl-2-butyl acetate}<smiles>CC(=O)OC(C)(C)C(C)(C)C</smiles>

GP1 was performed using 2,3,3-trimethyl-2-butanol (2.32 g, $20.0 \mathrm{mmol})$, acetic anhydride $(2.84 \mathrm{~mL}$, $30.0 \mathrm{mmol})$, pyridine $(4.0 \mathrm{~mL})$ and $\operatorname{DMAP}(0.244 \mathrm{~g}, 2.00 \mathrm{mmol})$. After $72 \mathrm{~h}$, a further portion of acetic anhydride $\left(5.68 \mathrm{~mL}, 60 \mathrm{mmol}\right.$ ) was added, and the mixture was stirred for a further $72 \mathrm{~h}$ at $100{ }^{\circ} \mathrm{C}$ until complete conversion was observed by ${ }^{1} \mathrm{H}$ NMR spectroscopy. After kugelrohr distillation $\left(40-45^{\circ} \mathrm{C}\right.$, $210 \mathrm{mbar}$ ), 2,3,3-trimethyl-2-butyl acetate (2.82 $\mathrm{g}, 17.8 \mathrm{mmol}, 89 \%)$ was obtained as a colourless liquid.

${ }^{1} \mathrm{H}$ NMR (500 MHz, CDCl $)$ : $\delta 1.97(\mathrm{~s}, 3 \mathrm{H}), 1.48(\mathrm{~s}, 6 \mathrm{H}), 0.95(\mathrm{~s}, 9 \mathrm{H})$.

${ }^{13} \mathrm{C}\left\{{ }^{1} \mathrm{H}\right\}$ NMR (126 MHz, $\left.\mathrm{CDCl}_{3}\right): \delta$ 170.7, 86.8, 38.2, 25.1, 22.6, 20.4.

$v_{\max }($ neat $) / \mathrm{cm}^{-1}: 2973,2917,2879,1731,1467,1367,1256,1218,1171,1134,1017,939,847,782$, $610,506,491$.

HRMS: calcd. for $\mathrm{C}_{9} \mathrm{H}_{18} \mathrm{O}_{2}+\mathrm{Na}^{+}$: $181.1199[\mathrm{M}+\mathrm{Na}]^{+}$; found $\left.(\mathrm{ESI})^{+}\right)$: 181.1182 . 


\section{2-Methyl-4-(4-bromophenyl)-2-butanol}<smiles>CCOC(=O)[C@H](OCC)OC(=O)CCc1ccc(Br)cc1</smiles>

Dimethylformamide (3 drops) was added to a solution of 3-(4-bromophenyl)propanoic acid (4.12 g, $18.0 \mathrm{mmol})$ and oxalyl chloride $(1.72 \mathrm{~mL}, 20.0 \mathrm{mmol})$ in anhydrous dichloromethane $(20 \mathrm{~mL})$ under dinitrogen. Vigorous effervescence was observed. The mixture was stirred at r.t. for $1.5 \mathrm{~h}$, then the solvent was removed in vacuo and anhydrous $\mathrm{Et}_{2} \mathrm{O}(20 \mathrm{~mL})$ was added to give a pale yellow solution. Methylmagnesium bromide ( $3.0 \mathrm{M}$ in $\mathrm{Et}_{2} \mathrm{O} ; 20.0 \mathrm{~mL}, 60.0 \mathrm{mmol}$ ) was added slowly and the resulting mixture was stirred at r.t. for $1 \mathrm{~h}$. A saturated aqueous solution of $\mathrm{NH}_{4} \mathrm{Cl}$ was added and the phases were separated. The aqueous phase was extracted with $\mathrm{Et}_{2} \mathrm{O}(3 \times)$ and the combined organic portions were dried $\left(\mathrm{MgSO}_{4}\right)$, filtered and the solvent was removed in vacuo to give 2-methyl-4-(4bromophenyl)-2-butanol (4.01 g, $16.5 \mathrm{mmol}, 92 \%)$ as a brown oil which solidified on standing. This material was used without further purification.

Characterisation data were consistent with literature values: ${ }^{1} \mathrm{H}^{2}$ and ${ }^{13} \mathrm{C}\left\{{ }^{1} \mathrm{H}\right\}^{2} \mathrm{NMR}$.

${ }^{1} \mathrm{H}$ NMR $\left(500 \mathrm{MHz}, \mathrm{CDCl}_{3}\right): \delta$ 7.41-7.38 $(\mathrm{m}, 2 \mathrm{H})$, 7.09-7.06 (m, 2H), 2.68-2.64 (m, 2H), 1.78-1.73 (m, $2 \mathrm{H}), 1.29(\mathrm{~s}, 6 \mathrm{H}), 1.21(\mathrm{~s}, 1 \mathrm{H})$.

${ }^{13} \mathrm{C}\left\{{ }^{1} \mathrm{H}\right\}$ NMR (126 MHz, $\left.\mathrm{CDCl}_{3}\right): \delta 141.7,131.6,130.2,119.6,70.9,45.7,30.3,29.5$.

$V_{\max }($ neat $) / \mathrm{cm}^{-1}: 3288,2969,2929,2863,1486,1402,1379,1362,1262,1206,1154,1124,1091,1070$, $1010,908,834,801,761,631,521,484,452$.

HRMS: calcd. for $\mathrm{C}_{11} \mathrm{H}_{15}{ }^{79} \mathrm{BrO}+\mathrm{Na}^{+}: 265.0198[\mathrm{M}+\mathrm{Na}]^{+}$; found (ESI $\left.{ }^{+}\right): 265.0193$.

m.p./ ${ }^{\circ} \mathrm{C}: 35-38$, lit. $^{2} 34-37$. 


\section{2-Methyl-4-(4-bromophenyl)-2-butyl acetate}<smiles>CC(=O)OC(C)(C)CCc1ccc(Br)cc1</smiles>

GP1 was performed using 2-methyl-4-(4-bromophenyl)-2-butanol (3.65 g, $15.0 \mathrm{mmol}$ ), acetic anhydride $(2.13 \mathrm{~mL}, 22.5 \mathrm{mmol})$, pyridine $(3.0 \mathrm{~mL})$ and DMAP $(0.183 \mathrm{~g}, 1.50 \mathrm{mmol})$. After kugelrohr distillation (200-220 ${ }^{\circ} \mathrm{C}, 0.3 \mathrm{mbar}$ ), 2-methyl-4-(4-bromophenyl)-2-butyl acetate (3.84 g, $13.5 \mathrm{mmol}$, $90 \%)$ was obtained as a yellow liquid.

${ }^{1} \mathrm{H}$ NMR (500 MHz, $\left.\mathrm{CDCl}_{3}\right): \delta$ 7.40-7.37 (m, 2H), 7.08-7.04 (m, 2H), 2.61-2.56 (m, 2H), 2.05-2.00 (m, 2H), $1.97(\mathrm{~s}, 3 \mathrm{H}), 1.49(\mathrm{~s}, 6 \mathrm{H})$.

${ }^{13} \mathrm{C}\left\{{ }^{1} \mathrm{H}\right\}$ NMR $\left(126 \mathrm{MHz}, \mathrm{CDCl}_{3}\right): \delta 170.6,141.2,135.6,130.3,119.7,81.9,42.6,30.0,26.3,22.5$.

$v_{\max }($ neat $) / \mathrm{cm}^{-1}: 2975,2931,2870,1729,1488,1455,1366,1246,1201,1168,1125,1094,1071,1011$, $945,828,803,761,609,519,466$.

HRMS: calcd. for $\mathrm{C}_{13} \mathrm{H}_{17}{ }^{79} \mathrm{BrO}_{2}+\mathrm{Na}^{+}$: $307.0304[\mathrm{M}+\mathrm{Na}]^{+}$; found $(\mathrm{ESI})^{+}$): 307.0303 . 


\section{1-Methylcyclopentyl acetate}

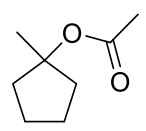

GP1 was performed using 1-methylcyclopentanol $(5.01 \mathrm{~g}, 50.0 \mathrm{mmol})$, acetic anhydride $(7.10 \mathrm{~mL}, 75.0$ $\mathrm{mmol})$, pyridine $(6.0 \mathrm{~mL}$ ) and DMAP $(0.611 \mathrm{~g}, 5.00 \mathrm{mmol})$. After kugelrohr distillation (r.t., $0.3 \mathrm{mbar})$, 1-methylcyclopentyl acetate $(6.23 \mathrm{~g}, 46.8 \mathrm{mmol}, 88 \%)$ was obtained as a colourless liquid.

Characterisation data were consistent with literature values: ${ }^{1} \mathrm{H}^{3}$ and ${ }^{13} \mathrm{C}\left\{{ }^{1} \mathrm{H}\right\}^{3} \mathrm{NMR}$.

${ }^{1} \mathrm{H}$ NMR $\left(500 \mathrm{MHz}, \mathrm{CDCl}_{3}\right): \delta$ 2.10-2.02 (m, 2H), $1.95(\mathrm{~s}, 3 \mathrm{H}), 1.73-1.62(\mathrm{~m}, 4 \mathrm{H}), 1.62-1.56(\mathrm{~m}, 2 \mathrm{H}), 1.52$ $(\mathrm{s}, 3 \mathrm{H})$.

${ }^{13} \mathrm{C}\left\{{ }^{1} \mathrm{H}\right\}$ NMR (126 MHz, $\left.\mathrm{CDCl}_{3}\right): \delta 170.8,89.8,39.2,24.4,23.9,22.5$.

$\mathbf{V}_{\max }\left(\right.$ neat)$/ \mathrm{cm}^{-1}:$ 2966, 2874, 1731, 1443, 1367, 1337, 1249, 1188, 1122, 1018, 939, 855, 613, 461.

HRMS: Neither the parent ion nor any obvious fragments could be observed by ESI-MS. 


\section{1-Methylcyclohexyl acetate}<smiles>CC(=O)OC1(C)CCCCC1</smiles>

GP1 was performed using 1-methylcyclohexanol $(6.21 \mathrm{~mL}, 50.0 \mathrm{mmol})$, acetic anhydride $(5.20 \mathrm{~mL}, 55.0$ $\mathrm{mmol}$ ) and pyridine $(6.0 \mathrm{~mL}$ ). After kugelrohr distillation (r.t., $0.2 \mathrm{mbar}$ ) 1-methylcyclohexyl acetate $(6.87 \mathrm{~g}, 44.0 \mathrm{mmol}, 88 \%)$ was obtained as a colourless liquid.

Characterisation data were consistent with literature values: ${ }^{1} \mathrm{H}^{4}$ and ${ }^{13} \mathrm{C}\left\{{ }^{1} \mathrm{H}\right\}^{5} \mathrm{NMR}, \mathrm{IR} .{ }^{5}$

${ }^{1} \mathrm{H}$ NMR (400 MHz, $\left.\mathrm{CDCl}_{3}\right): \delta$ 2.15-2.08 (m, 2H), $2.00(\mathrm{~s}, 3 \mathrm{H}), 1.58-1.22(\mathrm{~m}, 11 \mathrm{H})$.

${ }^{13} \mathrm{C}\left\{{ }^{1} \mathrm{H}\right\}$ NMR (101 MHz, $\left.\mathrm{CDCl}_{3}\right): \delta$ 170.6, 81.9, 36.8, 25.6, 25.5, 22.6, 22.3.

$v_{\max }($ neat $) / \mathrm{cm}^{-1}:$ 2932, 2861, 1730, 1448, 1367, 1285, 1266, 1232, 1155, 1107, 10431, 1017, 964, 945 , $918,870,809,611,467$.

HRMS: Neither the parent ion nor any obvious fragments could be observed by ESI-MS. 


\section{1-Phenylcyclohexyl acetate}

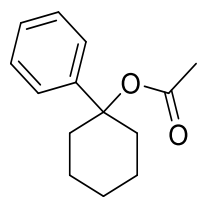

GP1 was performed using 1-phenylcyclohexanol (3.52 g, $20.0 \mathrm{mmol})$, acetic anhydride (2.84 mL, 30.0 $\mathrm{mmol})$, pyridine $(4.0 \mathrm{~mL})$ and DMAP $(0.244 \mathrm{~g}, 2.00 \mathrm{mmol})$. After kugelrohr distillation $\left(120^{\circ} \mathrm{C}, 0.3-0.4\right.$ mbar), 1-phenylcyclohexyl acetate (3.64 g, $16.7 \mathrm{mmol}, 83 \%$ ) was obtained as a pale yellow liquid which solidified on standing.

${ }^{1} \mathrm{H}$ NMR $\left(500 \mathrm{MHz}, \mathrm{CDCl}_{3}\right): \delta$ 7.37-7.30 (m, 4H), 7.25-7.21 (m, 1H), 2.52-2.46 (m, 2H), $2.05(\mathrm{~s}, 3 \mathrm{H}), 1.78-$ $1.70(\mathrm{~m}, 3 \mathrm{H}), 1.69-1.63(\mathrm{~m}, 4 \mathrm{H}), 1.35-1.25(\mathrm{~m}, 1 \mathrm{H})$.

${ }^{13} \mathrm{C}\left\{{ }^{1} \mathrm{H}\right\}$ NMR $\left(126 \mathrm{MHz}, \mathrm{CDCl}_{3}\right): \delta$ 169.7, 145.8, 128.4, 127.1, 124.7, 82.6, 36.4, 25.5, 22.3, 22.2.

$\mathbf{V}_{\max }($ neat $) / \mathrm{cm}^{-1}:$ 3087, 3059, 3028, 2933, 2860, 1737, 1494, 1448, 1366, 1263, 1227, 1132, 1013, 960 , $910,843,817,757,696,646,630,600,537$.

HRMS: calcd. for $\mathrm{C}_{14} \mathrm{H}_{18} \mathrm{O}_{2}+\mathrm{Na}^{+}: 241.1199[\mathrm{M}+\mathrm{Na}]^{+}$; found $\left(\mathrm{ESI}^{+}\right)$: 241.1196.

m.p./ ${ }^{\circ} \mathrm{C}:$ 57-59, lit. ${ }^{6}$ 58-59. 


\section{1-Adamantyl acetate}

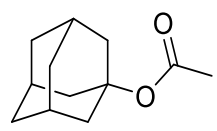

GP1 was performed using 1-adamantanol $(7.61 \mathrm{~g}, 50.0 \mathrm{mmol})$, acetic anhydride $(7.10 \mathrm{~mL}, 75.0 \mathrm{mmol})$, pyridine $(8.0 \mathrm{~mL})$ and DMAP $(0.612 \mathrm{~g}, 5.00 \mathrm{mmol})$. After kugelrohr distillation (135-140 $\left.{ }^{\circ} \mathrm{C}, 6-8 \mathrm{mbar}\right)$ 1-adamantyl acetate $(9.71 \mathrm{~g}, 50.0 \mathrm{mmol},>99 \%)$ was obtained as a colourless liquid.

Characterisation data were consistent with literature values: ${ }^{1} \mathrm{H}^{5}$ and ${ }^{13} \mathrm{C}\left\{{ }^{1} \mathrm{H}\right\}^{5} \mathrm{NMR}, \mathrm{IR} .{ }^{5}$

${ }^{1} \mathrm{H}$ NMR $\left(400 \mathrm{MHz}, \mathrm{CDCl}_{3}\right): \delta$ 2.18-2.12 (m, 3H), 2.12-2.09 (m, 6H), $1.98(\mathrm{~s}, 3 \mathrm{H}), 1.72-1.64(\mathrm{~m}, 6 \mathrm{H})$.

${ }^{13} \mathrm{C}\left\{{ }^{1} \mathrm{H}\right\}$ NMR (101 MHz, $\left.\mathrm{CDCl}_{3}\right): \delta 170.4,80.4,41.4,36.4,30.9,22.9$.

$v_{\max }($ neat $) / \mathrm{cm}^{-1}:$ 2910, 2853, 1731, 1456, 1367, 1354, 1241, 1059, 1016, 864.

HRMS: calcd. For $\mathrm{C}_{12} \mathrm{H}_{18} \mathrm{O}_{2}+\mathrm{Na}^{+}: 217.1199[\mathrm{M}+\mathrm{Na}]^{+}$; found $\left.(\mathrm{ESI})^{+}\right): 217.1192$.

m.p./ ${ }^{\circ} \mathrm{C}: 31-32$, lit. $^{5} 31$. 


\section{1-Adamantyl pivalate}

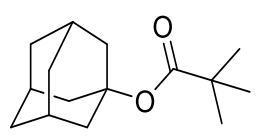

GP1 was performed using 1-adamantanol (3.04 g, $20.0 \mathrm{mmol})$, pivalic anhydride (5.59 mL, $30.0 \mathrm{mmol})$, pyridine $(4.0 \mathrm{~mL})$, and DMAP $(0.244 \mathrm{~g}, 2.00 \mathrm{mmol})$. After stirring for 1 week, the crude product was obtained as a mixture with $\mathrm{Piv}_{2} \mathrm{O}$ (1.0:0.5). Kugelrohr distillation was used to separate the two species (Piv $2 \mathrm{O}: 50-60{ }^{\circ} \mathrm{C}, 0.5 \mathrm{mbar}$; 1-adamantyl pivalate: $120-126{ }^{\circ} \mathrm{C}, 0.2 \mathrm{mbar}$ ). 1-Adamantyl pivalate (4.01 g, $17.0 \mathrm{mmol}, 85 \%)$ containing $<5 \% \mathrm{Piv}_{2} \mathrm{O}$ was obtained in this manner as a colourless liquid.

Characterisation data were consistent with literature values: ${ }^{1} \mathrm{H}^{7}$ and ${ }^{13} \mathrm{C}\left\{{ }^{1} \mathrm{H}\right\}{ }^{7} \mathrm{NMR}, \mathrm{IR} .{ }^{8}$

${ }^{1} \mathrm{H}$ NMR $\left(500 \mathrm{MHz}, \mathrm{CDCl}_{3}\right): \delta$ 2.18-2.13 (m, 3H), 2.11-2.07 (m, 6H), 1.69-1.63 (m, 6H), $1.14(\mathrm{~s}, 9 \mathrm{H})$.

${ }^{13} \mathrm{C}\left\{{ }^{1} \mathrm{H}\right\}$ NMR (126 MHz, $\left.\mathrm{CDCl}_{3}\right): \delta$ 178.0, 79.7, 41.4, 39.4, 36.4, 30.9, 27.4.

$v_{\max }($ neat $) / \mathrm{cm}^{-1}:$ 2909, 2853, 1722, 1479, 1457, 1394, 1355, 1318, 1281, 1161, 1103, 1056, 1004, 969, $940,917,872,814,770,573,427,407$.

HRMS: calcd. For $\mathrm{C}_{15} \mathrm{H}_{24} \mathrm{O}_{2}+\mathrm{Na}^{+}: 259.1669[\mathrm{M}+\mathrm{Na}]^{+}$; found (ESI $\left.{ }^{+}\right): 259.1673$. 


\section{1-Adamantyl benzoate}

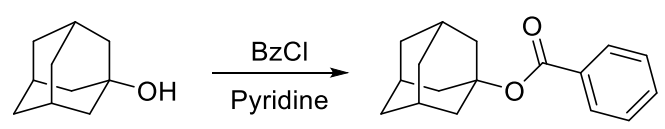

Benzoyl chloride (1.27 mL, $11.0 \mathrm{mmol})$ was added to a solution of 1-adamantanol (1.52 g, $10.0 \mathrm{mmol})$ in pyridine $(5.0 \mathrm{~mL})$ at r.t. The mixture was stirred at r.t. for $16 \mathrm{~h}$, then an aqueous solution of $\mathrm{NaHCO}_{3}$ $(5 \% \mathrm{w} / \mathrm{v}, 25 \mathrm{~mL})$ was added. The aqueous phase was separated and extracted with dichloromethane $(3 \times 20 \mathrm{~mL})$, the organic portions were combined and washed with brine $(3 \times 20 \mathrm{~mL})$, dried $\left(\mathrm{MgSO}_{4}\right)$, filtered and concentrated in vacuo to give the crude product as a colourless solid. Recrystallisation from hot methanol gave 1-adamantyl benzoate $(2.08 \mathrm{~g}, 8.12 \mathrm{mmol}, 81 \%)$ as a colourless crystalline solid.

Characterisation data were consistent with literature values: ${ }^{1} \mathrm{H}^{9}$ and ${ }^{13} \mathrm{C}\left\{{ }^{1} \mathrm{H}\right\}^{9} \mathrm{NMR}, \mathrm{IR} .{ }^{10}$

${ }^{1} \mathrm{H}$ NMR $\left(400 \mathrm{MHz}, \mathrm{CDCl}_{3}\right): \delta 8.01-7.96(\mathrm{~m}, 2 \mathrm{H}), 7.52(\mathrm{tt}, J=7.3,1.4 \mathrm{~Hz}, 1 \mathrm{H}), 7.44-7.38(\mathrm{~m}, 2 \mathrm{H}), 2.28-$ $2.25(\mathrm{~m}, 6 \mathrm{H}), 2.25-2.19(\mathrm{~m}, 3 \mathrm{H}), 1.76-1.67(\mathrm{~m}, 6 \mathrm{H})$.

${ }^{13} \mathrm{C}\left\{{ }^{1} \mathrm{H}\right\}$ NMR $\left(101 \mathrm{MHz}, \mathrm{CDCl}_{3}\right): \delta 165.6,132.5,132.3,129.6,128.3,81.2,41.6,36.4,31.1$.

$V_{\max }($ neat $) / \mathrm{cm}^{-1}: 2907,2847,1704,1453,1321,1271,1178,1117,1103,1070,1048,1027,711,685$.

HRMS: calcd. for $\mathrm{C}_{17} \mathrm{H}_{20} \mathrm{O}_{2}+\mathrm{Na}^{+}: 279.1356[\mathrm{M}+\mathrm{Na}]^{+}$; found $\left.(\mathrm{ESI})^{+}\right): 279.1357$.

m.p./ ${ }^{\circ} \mathrm{C}:$ 64-65, lit. ${ }^{11} 66.5-67$. 


\section{2-Methyl-2-adamantanol ${ }^{12}$}

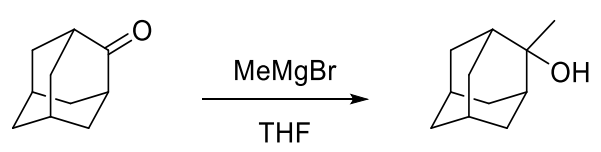

Methylmagnesium bromide $\left(3.0 \mathrm{M}\right.$ in $\mathrm{Et}_{2} \mathrm{O} ; 10.0 \mathrm{~mL}, 30.0 \mathrm{mmol}$ ) was added to a solution of 2-adamantanone $(2.25 \mathrm{~g}, 15.0 \mathrm{mmol})$ in anhydrous THF $(20 \mathrm{~mL})$ at $0{ }^{\circ} \mathrm{C}$. The mixture was stirred at 0 ${ }^{\circ} \mathrm{C}$ for $1 \mathrm{~h}$ before a saturated aqueous solution of $\mathrm{NH}_{4} \mathrm{Cl}$ was added. The phases were separated and the aqueous phase was extracted with $\mathrm{Et}_{2} \mathrm{O}(3 \times)$. The organic layers were combined, dried $\left(\mathrm{MgSO}_{4}\right)$, filtered and the solvent was removed in vacuo to give 2-methyl-2-adamantanol (2.41 g, $14.5 \mathrm{mmol}$, $97 \%)$ as a colourless solid.

Characterisation data were consistent with literature values: ${ }^{1} \mathrm{H}^{13}$ and ${ }^{13} \mathrm{C}\left\{{ }^{1} \mathrm{H}\right\}^{13} \mathrm{NMR}$.

${ }^{1} \mathrm{H}$ NMR $\left(500 \mathrm{MHz}, \mathrm{CDCl}_{3}\right): \delta$ 2.21-2.15 $(\mathrm{m}, 2 \mathrm{H}), 1.89-1.84(\mathrm{~m}, 2 \mathrm{H}), 1.82-1.77(\mathrm{~m}, 2 \mathrm{H}), 1.76-1.71(\mathrm{~m}$, $2 \mathrm{H}), 1.69-1.65(\mathrm{~m}, 4 \mathrm{H}), 1.58-1.53(\mathrm{~m}, 2 \mathrm{H}), 1.42(\mathrm{~s}, 1 \mathrm{H}), 1.34(\mathrm{~s}, 3 \mathrm{H})$.

${ }^{13} \mathrm{C}\left\{{ }^{1} \mathrm{H}\right\}$ NMR (126 MHz, $\left.\mathrm{CDCl}_{3}\right): \delta 74.0,39.3,38.4,35.3,33.1,27.7,27.5,27.2$.

$v_{\max }($ neat $) / \mathrm{cm}^{-1}: 3355,2989,2888,2852,1442,1374,1353,1334,1207,1121,1079,1045,1029,953$, $916,870,825,802,607,492,468,446$.

HRMS: calcd. for $\mathrm{C}_{11} \mathrm{H}_{18} \mathrm{O}+\mathrm{Na}^{+}$: $189.1250[\mathrm{M}+\mathrm{Na}]^{+}$; found $\left.(\mathrm{ESI})^{+}\right)$: 189.1236 .

m.p./ ${ }^{\circ}$ C: 203-206, lit. ${ }^{13} 207$. 


\section{2-Methyl-2-adamantyl acetate}

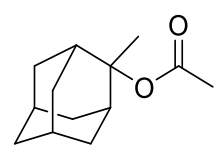

GP1 was performed using 2-methyl-2-adamantanol $(2.00 \mathrm{~g}, 12.0 \mathrm{mmol})$, acetic anhydride $(1.70 \mathrm{~mL}$, $18.0 \mathrm{mmol})$, pyridine $(3.0 \mathrm{~mL})$ and DMAP $(0.147 \mathrm{~g}, 1.20 \mathrm{mmol})$. After kugelrohr distillation $\left(100^{\circ} \mathrm{C}, 0.2-\right.$ $0.3 \mathrm{mbar}$ ), 2-methyl-2-adamantyl acetate (2.27 g, $10.9 \mathrm{mmol}, 91 \%)$ was obtained as a colourless liquid.

Characterisation data were consistent with literature values: ${ }^{1} \mathrm{H}^{14}$ and ${ }^{13} \mathrm{C}\left\{{ }^{1} \mathrm{H}\right\}^{14} \mathrm{NMR}$.

${ }^{1} \mathrm{H}$ NMR $\left(500 \mathrm{MHz}, \mathrm{CDCl}_{3}\right): \delta 2.28-2.25(\mathrm{~m}, 2 \mathrm{H}), 2.03-1.98(\mathrm{~m}, 2 \mathrm{H}$ and s, $3 \mathrm{H}), 1.89-1.84(\mathrm{~m}, 2 \mathrm{H}), 1.81-$ $1.77(\mathrm{~m}, 2 \mathrm{H}), 1.74-1.72(\mathrm{~m}, 1 \mathrm{H}), 1.72-1.68(\mathrm{~m}, 3 \mathrm{H}), 1.61(\mathrm{~s}, 3 \mathrm{H}), 1.58-1.56(\mathrm{~m}, 1 \mathrm{H}), 1.56-1.53(\mathrm{~m}, 1 \mathrm{H})$.

${ }^{13} \mathrm{C}\left\{{ }^{1} \mathrm{H}\right\}$ NMR $\left(126 \mathrm{MHz}, \mathrm{CDCl}_{3}\right): \delta 170.4,86.9,38.3,36.3,34.6,33.2,27.4,26.7,22.4,22.2$.

$v_{\max }($ neat $) / \mathrm{cm}^{-1}: 2902,2860,1726,1446,1363,1244,1218,1104,1025,956,935,887,841,609,504$, 456.

HRMS: calcd. for $\mathrm{C}_{13} \mathrm{H}_{20} \mathrm{O}_{2}+\mathrm{Na}^{+}: 231.1356\left[\mathrm{M}+\mathrm{Na}^{+}\right.$; found $\left(\mathrm{ESI}^{+}\right): 231.1347$. 


\section{(+)-Cedryl acetate}

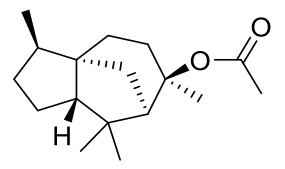

GP1 was performed using (+)-cedrol $(4.45 \mathrm{~g}, 20.0 \mathrm{mmol})$, acetic anhydride $(2.84 \mathrm{~mL}, 30.0 \mathrm{mmol})$, pyridine $(4.0 \mathrm{~mL})$ and DMAP $(0.244 \mathrm{~g}, 2.00 \mathrm{mmol})$. After kugelrohr distillation (130-140 $\left.{ }^{\circ} \mathrm{C}, 0.1 \mathrm{mbar}\right)$, (+)-cedryl acetate $(5.20 \mathrm{~g}, 19.7 \mathrm{mmol}, 98 \%)$ was obtained as a pale yellow liquid, which solidified on standing.

Characterisation data were consistent with literature values: ${ }^{1} \mathrm{H}^{15}$ and ${ }^{13} \mathrm{C}\left\{{ }^{1} \mathrm{H}\right\}^{15}$ NMR. Note: the literature ${ }^{1} \mathrm{H}$ NMR spectroscopic data only accounts for 25 of the 28 expected protons.

${ }^{1} \mathrm{H}$ NMR $\left(500 \mathrm{MHz}, \mathrm{CDCl}_{3}\right): \delta 2.39(\mathrm{dd}, J=5.2,1.5 \mathrm{~Hz}, 1 \mathrm{H}), 2.03(\mathrm{ddt}, J=13.7,5.7,1.7 \mathrm{~Hz}, 1 \mathrm{H}), 1.96-$ $1.83(\mathrm{~m}, 5 \mathrm{H}$ including a singlet at $1.95 \mathrm{ppm}), 1.82-1.78(\mathrm{~m}, 1 \mathrm{H}), 1.68-1.60(\mathrm{~m}, 2 \mathrm{H}), 1.55-1.49(\mathrm{~m}, 4 \mathrm{H}$ including a singlet at $1.54 \mathrm{ppm}), 1.46-1.23(\mathrm{~m}, 5 \mathrm{H}), 1.17(\mathrm{~s}, 3 \mathrm{H}), 0.97(\mathrm{~s}, 3 \mathrm{H}), 0.83(\mathrm{~d}, J=7.1 \mathrm{~Hz}, 3 \mathrm{H})$.

${ }^{13} \mathrm{C}\left\{{ }^{1} \mathrm{H}\right\}$ NMR (126 MHz, CDCl 3$): \delta 170.5,86.4,57.0,56.8,54.1,43.5,41.5,41.1,37.1,33.3,31.4,28.6$, $27.1,26.0,25.4,22.9,15.7$.

$v_{\max }($ neat $) / \mathrm{cm}^{-1}: 2963,2952,2934,2901,2872,1722,1471,1457,1364,1262,1242,1117,1086,1021$, $949,927,840,757,696,612,461$.

HRMS: calcd. for $\mathrm{C}_{17} \mathrm{H}_{28} \mathrm{O}_{2}+\mathrm{Na}^{+}: 287.1982[\mathrm{M}+\mathrm{Na}]^{+}$; found $\left.(\mathrm{ESI})^{+}\right): 287.1981$.

m.p./ ${ }^{\circ} \mathrm{C}: 40-42$, lit. ${ }^{15} 44-46$. 


\section{2-Methyl-4-phenyl-2-butanol}<smiles>CCOC(C)(O)CCc1ccccc1</smiles>

Methylmagnesium bromide (3.0 M in Et $\mathrm{E}_{2} \mathrm{O} ; 10.0 \mathrm{~mL}, 30.0 \mathrm{mmol}$ ) was added dropwise to a solution of 3-phenylpropionyl chloride $(1.49 \mathrm{~mL}, 10.0 \mathrm{mmol})$ in anhydrous $\mathrm{Et}_{2} \mathrm{O}(20 \mathrm{~mL})$ at r.t. The mixture was stirred at r.t. for $3 \mathrm{~h}$, then a saturated aqueous solution of $\mathrm{NH}_{4} \mathrm{Cl}$ was added. The phases were separated and the aqueous phase was extracted with $\mathrm{Et}_{2} \mathrm{O}(3 \times)$. The combined organic layers were dried $\left(\mathrm{MgSO}_{4}\right)$, filtered and the solvent was removed in vacuo to give 2-methyl-4-phenyl-2-butanol (1.62 g, $9.86 \mathrm{mmol}, 99 \%)$ as a colourless liquid.

Characterisation data were consistent with literature values: ${ }^{1} \mathrm{H}^{16}$ and ${ }^{13} \mathrm{C}\left\{{ }^{1} \mathrm{H}\right\}{ }^{16} \mathrm{NMR}$.

${ }^{1} \mathrm{H}$ NMR $\left(500 \mathrm{MHz}, \mathrm{CDCl}_{3}\right): \delta$ 7.31-7.27 $(\mathrm{m}, 2 \mathrm{H}), 7.23-7.17(\mathrm{~m}, 3 \mathrm{H}), 2.74-2.69(\mathrm{~m}, 2 \mathrm{H}), 1.83-1.78(\mathrm{~m}$, 2H), 1.30 (app. s, 7H).

${ }^{13} \mathrm{C}\left\{{ }^{1} \mathrm{H}\right\}$ NMR (126 MHz, $\left.\mathrm{CDCl}_{3}\right): \delta 142.7,128.6,128.5,125.9,71.1,45.9,30.9,29.5$.

$v_{\max }($ neat $) / \mathrm{cm}^{-1}: 3383,3026,2968,2932,2864,1604,1494,1454,1376,1210,1149,1071,1031,913$, $829,767,738,697,519$.

HRMS: calcd. for $\mathrm{C}_{11} \mathrm{H}_{16} \mathrm{O}+\mathrm{NH}_{4}{ }^{+}: 182.1539\left[\mathrm{M}+\mathrm{NH}_{4}\right]^{+}$; found $\left(\mathrm{ESI}^{+}\right): 182.1534$. 


\section{2-Methyl-4-phenyl-2-butyl acetate $\left(d_{0}-2\right)$}

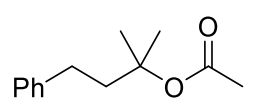

GP1 was performed using 2-methyl-4-phenyl-2-butanol (1.64 g, $10.0 \mathrm{mmol})$, acetic anhydride (1.42 $\mathrm{mL}, 15.0 \mathrm{mmol})$, pyridine $(2.0 \mathrm{~mL})$ and DMAP $(0.122 \mathrm{~g}, 1.00 \mathrm{mmol})$. After kugelrohr distillation (90-100 $\left.{ }^{\circ} \mathrm{C}, 0.05-0.06 \mathrm{mbar}\right), 2$-methyl-4-phenyl-2-butyl acetate $\left(\boldsymbol{d}_{0}-2\right)(1.86 \mathrm{~g}, 9.02 \mathrm{mmol}, 90 \%)$ was obtained as a colourless liquid.

Characterisation data were consistent with literature values: ${ }^{1} \mathrm{H}^{17}$ and ${ }^{13} \mathrm{C}\left\{{ }^{1} \mathrm{H}\right\}{ }^{17} \mathrm{NMR}, \mathrm{IR} \cdot{ }^{17}$

${ }^{1} \mathrm{H}$ NMR $\left(500 \mathrm{MHz}, \mathrm{CDCl}_{3}\right): \delta$ 7.30-7.26 (m, 2H), 7.21-7.17 (m, 3H), 2.67-2.62 (m, 2H), 2.10-2.05 (m, $2 \mathrm{H}), 1.98(\mathrm{~s}, 3 \mathrm{H}), 1.51(\mathrm{~s}, 6 \mathrm{H})$.

${ }^{13} \mathrm{C}\left\{{ }^{1} \mathrm{H}\right\}$ NMR (126 MHz, $\left.\mathrm{CDCl}_{3}\right): \delta$ 170.6, 142.2, 128.51, 128.50, 125.9, 82.1, 42.8, 30.5, 26.2, 22.5.

$v_{\max }($ neat $) / \mathrm{cm}^{-1}:$ 3027, 2977, 2936, 2867, 1730, 1604, 1495, 1454, 1366, 1246, 1202, 1169, 1123, 1073, $1017,945,822,765,742,698,611,517,464$.

HRMS: calcd. for $\mathrm{C}_{13} \mathrm{H}_{18} \mathrm{O}_{2}+\mathrm{Na}^{+}: 229.1199[\mathrm{M}+\mathrm{Na}]^{+}$; found $\left(\mathrm{ESI}^{+}\right)$: 229.1200. 


\section{2-Methyl-4-phenyl-2-butanol- $d_{6}$}

$\mathrm{Ph} \sim \mathrm{Br} \underset{\mathrm{Et}_{2} \mathrm{O}}{\stackrel{\mathrm{Mg} \mathrm{I}_{2}}{\longrightarrow}}[\mathrm{Ph} \sim \mathrm{MgBr}] \underset{\mathrm{Et}_{2} \mathrm{O}}{\stackrel{\mathrm{Acetone}^{-\mathrm{d}_{6}}}{\longrightarrow}} \mathrm{Ph}^{\stackrel{\mathrm{D}_{3} \mathrm{C}}{\mathrm{CD}_{3}}} \overbrace{\mathrm{OH}}^{\mathrm{CD}_{3}}$

A crystal of iodine was sublimed onto magnesium turnings ( $1.09 \mathrm{~g}, 45.0 \mathrm{mmol})$ under nitrogen, then anhydrous $\mathrm{Et}_{2} \mathrm{O}(30 \mathrm{~mL}$ ) was added. (2-Bromoethyl)benzene $(5.55 \mathrm{~g}, 30.0 \mathrm{mmol})$ was added dropwise with stirring to generate 2-phenylethylmagnesium bromide. This mixture was stirred for $0.5 \mathrm{~h}$ at r.t. before it was added slowly by cannula transfer to a solution of acetone- $d_{6}(1.84 \mathrm{~mL}, 25.0 \mathrm{mmol})$ in anhydrous $\mathrm{Et}_{2} \mathrm{O}(30 \mathrm{~mL})$. The mixture was stirred at r.t. for $1 \mathrm{~h}$, then a saturated aqueous solution of $\mathrm{NH}_{4} \mathrm{Cl}$ was added. The phases were separated and the aqueous phase was extracted with $\mathrm{Et}_{2} \mathrm{O}(3 \times)$. The organic layers were combined, dried $\left(\mathrm{MgSO}_{4}\right)$, filtered and the solvent was removed in vacuo. The crude material was layered onto a pad of silica gel which was eluted sequentially with pentane (to remove the 1,4-diphenylbutane side-product) and $\mathrm{Et}_{2} \mathrm{O}$ to give 2-methyl-4-phenyl-2-butanol- $d_{6}$ (3.50 g, $20.5 \mathrm{mmol}, 82 \%$, >99\% deuterium incorporation) as a colourless liquid.

${ }^{1} \mathrm{H}$ NMR $\left(500 \mathrm{MHz}, \mathrm{CDCl}_{3}\right): \delta$ 7.31-7.27 (m, 2H), 7.22-7.16 (m, 3H), 2.73-2.68 (m, 2H), 1.82-1.77 (m, $2 \mathrm{H}), 1.25(\mathrm{~s}, 1 \mathrm{H})$.

${ }^{13} \mathrm{C}\left\{{ }^{1} \mathrm{H}\right\}$ NMR (126 MHz, $\left.\mathrm{CDCl}_{3}\right): \delta$ 142.7, 128.6, 128.5, 125.9, 70.8, 45.8, 30.9, 28.5 (1:3:6:7:6:3:1 h, J = $19.2 \mathrm{~Hz})$.

$v_{\max }($ neat $) / \mathrm{cm}^{-1}: 3376,3027,2935,2863,2224,1603,1497,1454,1263,1175,1114,1050,956,812$, $743,696,577,509,465,430$.

HRMS: calcd. for $\mathrm{C}_{11} \mathrm{H}_{10} \mathrm{D}_{6} \mathrm{O}+\mathrm{Na}^{+}$: $193.1470[\mathrm{M}+\mathrm{Na}]^{+}$; found $\left(\mathrm{ESI}^{+}\right): 193.1477$. 


\section{2-Methyl-4-phenyl-2-butyl acetate- $d_{6}\left(d_{6}-2\right)$}<smiles>[2H]C([2H])([2H])C(CCc1ccccc1)(OC(C)=O)OC(C)=O</smiles>

GP1 was performed using 2-methyl-4-phenyl-2-butanol- $d_{6}(2.55 \mathrm{~g}, 15.0 \mathrm{mmol})$, acetic anhydride $(2.13 \mathrm{~mL}, 22.5 \mathrm{mmol})$, pyridine $(3.0 \mathrm{~mL})$ and DMAP $(0.183 \mathrm{~g}, 1.50 \mathrm{mmol})$. After kugelrohr distillation (90-100 $\left.{ }^{\circ} \mathrm{C}, 0.05-0.06 \mathrm{mbar}\right), 2$-methyl-4-phenyl-2-butyl acetate- $\boldsymbol{d}_{6}\left(\boldsymbol{d}_{6}-2\right)(3.06 \mathrm{~g}, 14.4 \mathrm{mmol}, 96 \%$, $>99 \%$ deuterium incorporation) was obtained as a colourless liquid.

${ }^{1} \mathrm{H}$ NMR $\left(500 \mathrm{MHz}, \mathrm{CDCl}_{3}\right): \delta$ 7.30-7.26 (m, 2H), 7.21-7.17 (m, 3H), 2.67-2.62 (m, 2H), 2.09-2.04 (m, 2H), $1.98(\mathrm{~s}, 3 \mathrm{H})$.

${ }^{13} \mathrm{C}\left\{{ }^{1} \mathrm{H}\right\}$ NMR (126 MHz, $\left.\mathrm{CDCl}_{3}\right): \delta$ 170.6, 142.3, 128.5, 128.5, 125.9, 81.8, 42.7, 30.4, 25.3 (1:3:6:7:6:3:1 $\mathrm{h}, J=19.2 \mathrm{~Hz}), 22.5$.

$v_{\max }($ neat $) / \mathrm{cm}^{-1}: 3027,2935,2866,2232,1728,1498,1454,1366,1252,1168,1104,1041,1018,963$, $923,809,745,697,607,509,462,436$.

HRMS: calcd. for $\mathrm{C}_{13} \mathrm{H}_{12} \mathrm{D}_{6} \mathrm{O}_{2}+\mathrm{Na}^{+}: 235.1576[\mathrm{M}+\mathrm{Na}]^{+}$; found $\left(\mathrm{ESI}{ }^{+}\right)$: 235.1576. 


\section{Determination of $\mathrm{Zn}_{3} \mathrm{P}_{2}$ Purity and Protonolysis Rate (Manuscript Scheme 2B)}

CAUTION: phosphine gas is highly toxic and is spontaneously flammable in air. Procedures that use phosphine gas should only be performed following appropriate training after consultation with local safety advisors, and after completing a thorough risk assessment. A syringe-stop should be employed to ensure that the barrel of the gas-syringe cannot be ejected during the titration.

Solid $\mathrm{Zn}_{3} \mathrm{P}_{2}$ (for mass see Figure S1) was added to a two-necked flask fitted with septa. The flask was evacuated and backfilled with anhydrous dinitrogen $(3 \times)$ before a gas syringe $(50 \mathrm{~mL})$ with a luer lock needle was inserted through one of the septa and purged with $N_{2}(3 \times)$. The flask was then disconnected from the Schlenk line and degassed aqueous $\mathrm{HCl}(5.0 \mathrm{M} ; 2.0 \mathrm{~mL}, 10 \mathrm{mmol})$ was added quickly in one portion using a Luer lock syringe. CAUTION: gas pressure within the apparatus increases immediately; the plunger of the syringe used to add hydrochloric acid should be held down after the acid has been added and while the syringe is removed carefully from the apparatus. The time taken for generation of gas was measured in $2.0 \mathrm{~mL}$ increments (taking into account the volume of hydrochloric acid $(2.0 \mathrm{~mL})$ that was added), and the mixture was stirred until no further gas was released. A solution of $\mathrm{KMnO}_{4}$ in aqueous sulfuric acid ( $0.50 \mathrm{M}$ in $0.50 \mathrm{M} \mathrm{H}_{2} \mathrm{SO}_{4}, 10 \mathrm{~mL}, 5.0 \mathrm{mmol}$ ) was added slowly, and the plunger of the gas syringe was driven back in to allow oxidation of the $\mathrm{PH}_{3}$ gas. CARE! exothermic reaction. After 10 minutes the vessel was opened to air.

Volumetric gas evolution data for two independent experiments are displayed in Figure S1. 


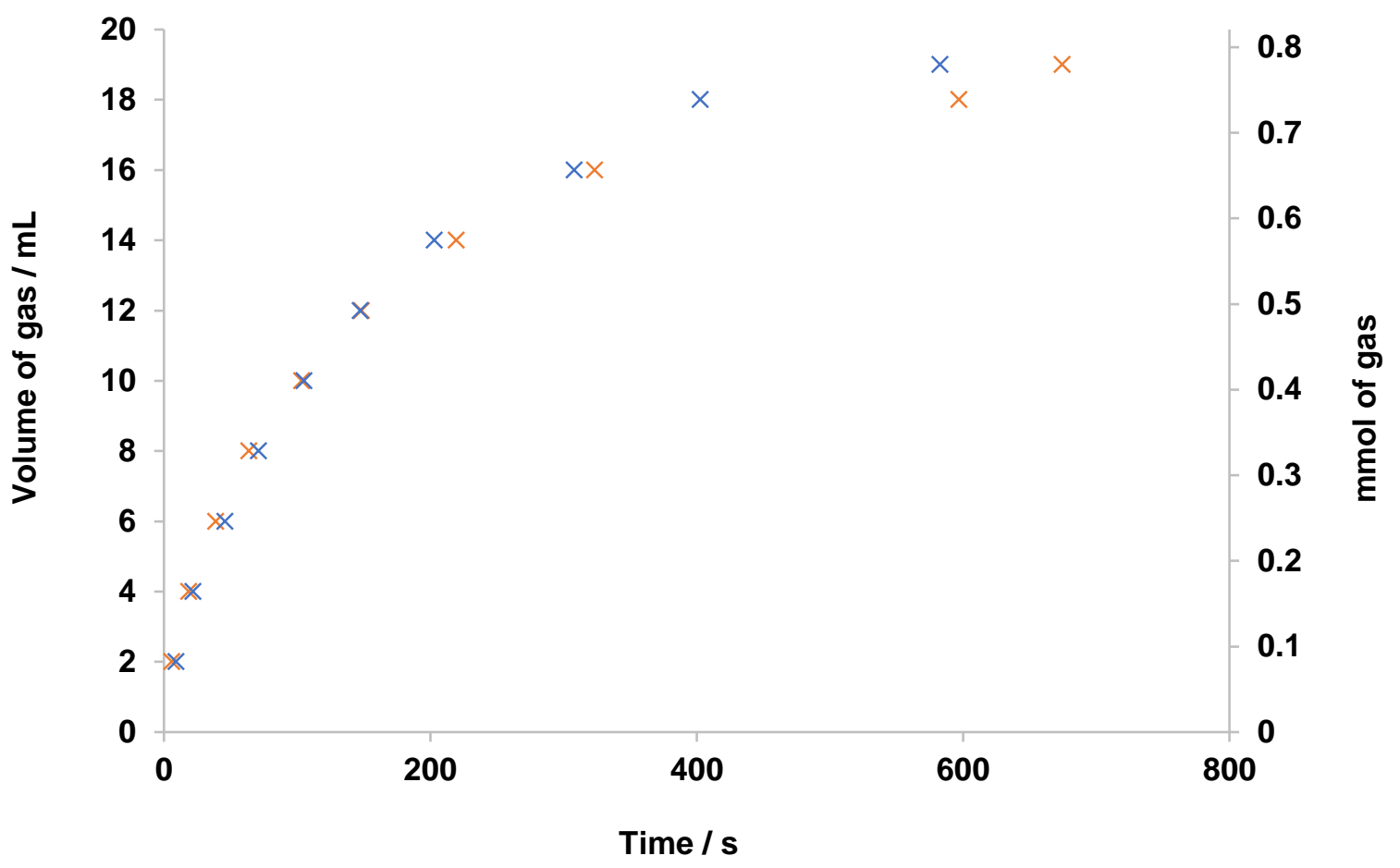

Figure S1. Volumetric gas titration of $\mathrm{Zn}_{3} \mathrm{P}_{2}$.

Blue - Run 1 ( $0.128 \mathrm{~g} \mathrm{Zn}_{3} \mathrm{P}_{2}, 0.496 \mathrm{mmol}$ at $100 \%$ purity): $19.0 \pm 0.5 \mathrm{~mL}$ gas evolved in $583 \mathrm{~s}$. Using $P V=n R T(P=1.00$ bar, $T=297 K) . n=0.780$ mmol gas. Purity $=78.6 \%$.

Orange - Run 2 (0.129 g Zn $\mathrm{P}_{2}, 0.500 \mathrm{mmol}$ at 100\% purity): $19.0 \pm 0.5 \mathrm{~mL}$ gas evolved in $674 \mathrm{~s}$. Using $P V=n R T(P=1.00$ bar, $T=297 K) . n=0.780$ mmol gas. Purity $=78.0 \%$. 


\section{Screening of Carbocation Sources (Manuscript Scheme 2C)}

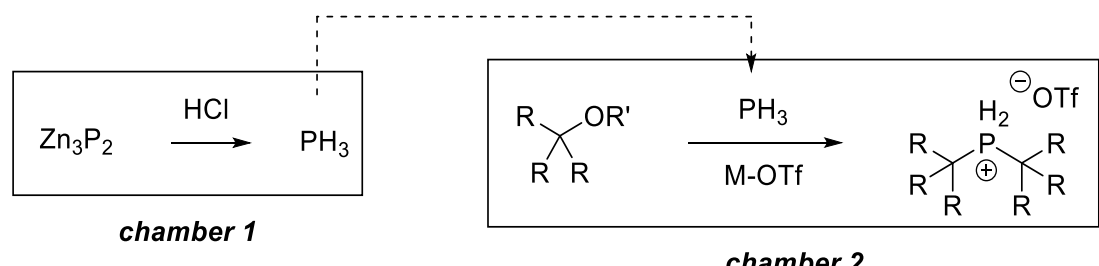

CAUTION: phosphine gas is highly toxic and is spontaneously flammable in air. Procedures that use phosphine gas should only be performed following appropriate training after consultation with local safety advisors, and after completing a thorough risk assessment. All CO-ware apparatus should be inspected for scratches or imperfections prior to use; new CO-ware septa should be used for each reaction, and the manufacturer's guidelines should be followed (no more than 2 injections per septum, using a needle with a diameter of $21 \mathrm{~g}(0.08 \mathrm{~mm})$ or less). CO-ware is recommended for routine use at pressures up to $46 \mathrm{psi}$, and not above $60 \mathrm{psi}$; this should be considered when changing reaction scales from those presented in this Supporting Information.

Zinc phosphide ( $78.0 \%$ purity, $0.165 \mathrm{~g}, 0.50 \mathrm{mmol}$ (to give $\left.1.00 \mathrm{mmol} \mathrm{PH}_{3}\right)$ ) was added to chamber one of a two-chamber reactor (CO-ware, $20 \mathrm{~mL}$ total volume, fitted with compatible septa). The reactor was evacuated and backfilled with anhydrous dinitrogen $(3 \times)$. Tert-amyl carbocation source $(6.00$ mmol, 6.00 equiv.) and trimethylsilyl trifluoromethanesulfonate $(0.181 \mathrm{~mL}, 1.00 \mathrm{mmol})$ or trifluoromethanesulfonic acid $(0.088 \mathrm{~mL}, 1.00 \mathrm{mmol})$ were added to chamber two. Degassed aqueous hydrochloric acid ( $5.0 \mathrm{M} ; 2.0 \mathrm{~mL}, 10 \mathrm{mmol})$ was added to chamber one quickly in a single portion using a Luer lock syringe. CAUTION: gas pressure within the apparatus increases immediately; the plunger of the syringe used to add hydrochloric acid should be held down after the acid has been added and while the syringe is removed carefully from the apparatus. The reaction was stirred at r.t. for $4 \mathrm{~h}$. The reactor was then opened to air and an aliquot was removed from chamber two for ${ }^{31} \mathrm{P}$ NMR spectroscopic analysis. 


\section{Synthesis of Di-tert-alkylphosphonium Salts $\underline{1}$ (Manuscript Scheme 3)}

General procedure - Synthesis of di-tert-alkylphosphonium salts (GP2)

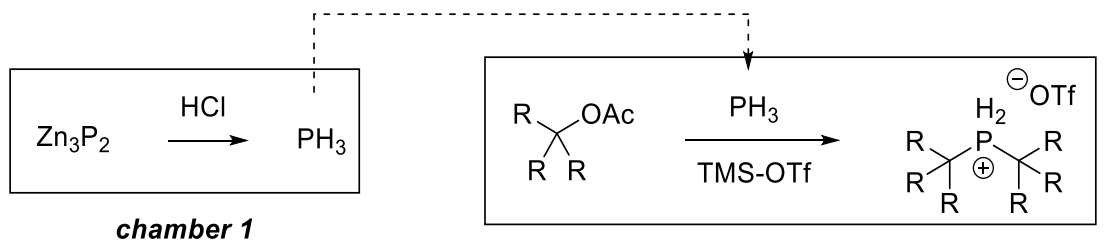

chamber 2

CAUTION: phosphine gas is highly toxic and is spontaneously flammable in air. Procedures that use phosphine gas should only be performed following appropriate training and after consultation with local safety advisors.

Zinc phosphide (78.0\% purity, $0.165 \mathrm{~g}, 0.50 \mathrm{mmol}$ (to give $\left.1.00 \mathrm{mmol} \mathrm{PH}_{3}\right)$ ) was added to chamber one of a two-chamber reactor (CO-ware, $20 \mathrm{~mL}$ total volume, fitted with compatible septa). The reactor was evacuated and backfilled with anhydrous dinitrogen $(3 \times)$. Tert-alkyl ester ( $3.00 \mathrm{mmol}, 3.00$ equiv.) and trimethylsilyl trifluoromethanesulfonate $(0.181 \mathrm{~mL}, 1.00 \mathrm{mmol})$ were added to chamber two. Degassed hydrochloric acid $(5.0 \mathrm{M} ; 2.0 \mathrm{~mL}, 10 \mathrm{mmol})$ was added to chamber one quickly in a single portion using a Luer lock syringe. CAUTION: gas pressure within the apparatus increases immediately; the plunger of the syringe used to add hydrochloric acid should be held down after the acid has been added and while the syringe is removed carefully from the apparatus. The reaction was stirred at r.t. for $4 \mathrm{~h}$. The reactor was then opened to air and washed into a flask with $\mathrm{Et}_{2} \mathrm{O}$. Products that spontaneously precipitated/crystallised from solution were isolated by filtration and washed with $\mathrm{Et}_{2} \mathrm{O}$. If the product did not spontaneously precipitate/crystallise, the reaction was concentrated in vacuo before $\mathrm{Et}_{2} \mathrm{O}$ was added; if no precipitation/crystallisation occurred at this point, mixtures were placed in a freezer at $-25^{\circ} \mathrm{C}$ overnight. The isolated phosphonium salts are stable under ambient laboratory conditions for at least a year. 


\section{Di-tert-amylphosphonium trifluoromethanesulfonate (1a)}

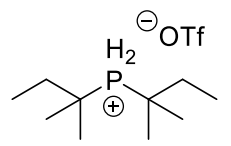

Conditions 1: GP2 was performed using tert-amyl acetate $(0.460 \mathrm{~mL}, 3.00 \mathrm{mmol})$. Di-tertamylphosphonium trifluoromethanesulfonate 1a $(0.151 \mathrm{~g}, 0.466 \mathrm{mmol}, 47 \%)$ was obtained as a colourless crystalline solid.

Conditions 2: GP2 was performed using tert-amyl acetate $(0.920 \mathrm{~mL}, 6.00 \mathrm{mmol})$. Di-tertamylphosphonium trifluoromethanesulfonate 1a $(0.249 \mathrm{~g}, 0.768 \mathrm{mmol}, 77 \%)$ was obtained as a colourless crystalline solid.

${ }^{1} \mathrm{H}$ NMR (500 MHz, CDCl $): \delta 6.41(\mathrm{~d}, J=472.5 \mathrm{~Hz}, 2 \mathrm{H}), 1.89(\mathrm{dq}, J=14.8,7.4 \mathrm{~Hz}, 4 \mathrm{H}), 1.54(\mathrm{~d}, J=18.5$ $\mathrm{Hz}, 12 \mathrm{H}), 1.08(\mathrm{t}, J=7.5 \mathrm{~Hz}, 6 \mathrm{H})$.

${ }^{13} \mathrm{C}\left\{{ }^{1} \mathrm{H}\right\}$ NMR (126 MHz, CDCl 3$): \delta 120.7(q, J=319.8 \mathrm{~Hz}), 35.5(\mathrm{~d}, J=33.6 \mathrm{~Hz}), 32.9,24.6,8.0(\mathrm{~d}, J=8.7$ $\mathrm{Hz})$.

${ }^{19} \mathrm{~F}$ NMR (470 MHz, $\left.\mathrm{CDCl}_{3}\right): \delta-78.47$.

31P NMR (202 MHz, $\left.\mathrm{CDCl}_{3}\right): \delta 15.25(\mathrm{tm}, J=472.6 \mathrm{~Hz})$.

$v_{\max }($ neat $) / \mathrm{cm}^{-1}: 2975,2424,1509,1468,1418,1250,1222,1153,1065,1026,939,867,828,812,772$, $755,702,675,635,572,516,494,418$.

HRMS: calcd. for $\mathrm{C}_{10} \mathrm{H}_{24} \mathrm{P}^{+}$: $175.1610[\mathrm{M}-\mathrm{OTf}]^{+}$; found $\left(\mathrm{ESI}^{+}\right)$: 175.1613 .

m.p./ ${ }^{\circ} \mathrm{C}: 113-115$. 
Di-(3-methyl-3-pentyl)phosphonium trifluoromethanesulfonate (1b)<smiles>CCC(CC)(CC)PC(CC)(CC)CC</smiles>

Conditions 1: GP2 was performed using 3-methyl-3-pentyl acetate (0.433 g, $3.00 \mathrm{mmol})$. Di-(3-methyl3-pentyl)phosphonium trifluoromethanesulfonate $1 \mathrm{~b}(0.159 \mathrm{~g}, 0.451 \mathrm{mmol}, 45 \%)$ was obtained as a colourless crystalline solid.

Conditions 2: GP2 was performed on a $5.0 \mathrm{mmol}$ scale in a $100 \mathrm{~mL}$ CO-ware reactor using $\mathrm{Zn}_{3} \mathrm{P}_{2}(78.0 \%$ purity, $0.827 \mathrm{~g}, 2.50 \mathrm{mmol}), 3$-methyl-3-pentyl acetate $(2.16 \mathrm{~g}, 15.0 \mathrm{mmol})$, trimethylsilyl trifluoromethanesulfonate $(0.905 \mathrm{~mL}, 5.00 \mathrm{mmol})$ and degassed hydrochloric acid $(5.0 \mathrm{M} ; 10 \mathrm{~mL}, 50$ mmol). Di-(3-methyl-3-pentyl)phosphonium trifluoromethanesulfonate $\mathbf{1 b}$ (1.14 g, 3.24 mmol, 65\%) was obtained as a colourless crystalline solid.

${ }^{1} \mathrm{H}$ NMR (500 MHz, CDCl 3 ): $\delta 6.36(\mathrm{~d}, J=470.1 \mathrm{~Hz}, 2 \mathrm{H}), 2.00-1.83(\mathrm{~m}, 8 \mathrm{H}), 1.54(\mathrm{~d}, J=18.7 \mathrm{~Hz}, 6 \mathrm{H}), 1.08$ $(\mathrm{t}, J=7.5 \mathrm{~Hz}, 12 \mathrm{H})$.

${ }^{13} \mathrm{C}\left\{{ }^{1} \mathrm{H}\right\}$ NMR $(126 \mathrm{MHz}, \mathrm{CDCl}$ ) $): \delta 120.7(\mathrm{q}, J=319.7 \mathrm{~Hz}), 39.8(\mathrm{~d}, J=32.0 \mathrm{~Hz}), 29.6,22.2,7.9(\mathrm{~d}, J=7.9$ $\mathrm{Hz})$.

${ }^{19}$ F NMR (470 MHz, $\left.\mathrm{CDCl}_{3}\right): \delta-78.42$.

${ }^{31}$ P NMR (202 MHz, $\left.\mathrm{CDCl}_{3}\right): \delta 3.45(\mathrm{tm}, J=470.3 \mathrm{~Hz})$.

$V_{\max }($ neat $) / \mathrm{cm}^{-1}: 2978,2949,2887,2410,1460,1394,1248,1222,1148,1027,939,754,635,571,516$.

HRMS: calcd. for $\mathrm{C}_{12} \mathrm{H}_{28} \mathrm{P}^{+}$: 203.1923 [M-OTf] ${ }^{+}$; found (ESI ${ }^{+}$): 203.1920.

m.p. $/{ }^{\circ} \mathrm{C}: 105-109$. 


\section{Di-(3-ethyl-3-pentyl)phosphonium trifluoromethanesulfonate (1c)}<smiles>CCC(CC)PC(CC)CC</smiles>

Conditions 1: GP2 was performed using 3-ethyl-3-pentyl acetate (0.475 g, $3.00 \mathrm{mmol})$. Di-(3-ethyl-3pentyl)phosphonium trifluoromethanesulfonate $1 \mathrm{c}(0.336 \mathrm{~g}, 0.883 \mathrm{mmol}, 88 \%)$ was obtained as a colourless crystalline solid.

Conditions 2: GP2 was performed on a $5.0 \mathrm{mmol}$ scale in a $100 \mathrm{~mL}$ CO-ware reactor using $\mathrm{Zn}_{3} \mathrm{P}_{2}(78.0 \%$ purity, $0.827 \mathrm{~g}, 2.50 \mathrm{mmol})$, 3-ethyl-3-pentyl acetate $(2.37 \mathrm{~g}, 15.0 \mathrm{mmol})$, trimethylsilyl trifluoromethanesulfonate $(0.91 \mathrm{~mL}, 5.0 \mathrm{mmol})$ and degassed hydrochloric acid $(5.0 \mathrm{M} ; 10 \mathrm{~mL}, 50$ mmol). Di-(3-ethyl-3-pentyl)phosphonium trifluoromethanesulfonate 1c (1.84 g, $4.84 \mathrm{mmol}, 97 \%$ ) was obtained as a colourless crystalline solid.

A triplet of 13 -ets is expected in the ${ }^{31} \mathrm{P}$ NMR spectrum, from ${ }^{1} J_{\mathrm{P}-\mathrm{H}}$ and ${ }^{3} \int_{\mathrm{P}-\mathrm{H}}$ couplings respectively. However, the lower intensity peaks of the multiplet are not observed due to poor signal:noise, and as such is reported below as a triplet of multiplets with both coupling constants given.

${ }^{1} \mathrm{H}$ NMR (500 MHz, CDCl 3 ): $\delta 6.21(\mathrm{~d}, J=468.9 \mathrm{~Hz}, 2 \mathrm{H}), 1.89$ (dq, $\left.J=17.4,7.4 \mathrm{~Hz}, 12 \mathrm{H}\right), 1.05(\mathrm{t}, J=7.4$ $\mathrm{Hz}, 18 \mathrm{H})$.

${ }^{13} \mathrm{C}\left\{{ }^{1} \mathrm{H}\right\}$ NMR $(126 \mathrm{MHz}, \mathrm{CDCl} 3): \delta 120.8(\mathrm{q}, J=320.1 \mathrm{~Hz}), 44.9(\mathrm{~d}, J=29.1 \mathrm{~Hz}), 27.2,8.0(\mathrm{~d}, J=7.1 \mathrm{~Hz})$. ${ }^{19}$ F NMR (470 MHz, $\left.\mathrm{CDCl}_{3}\right): \delta-78.38$.

31P NMR (202 MHz, $\left.\mathrm{CDCl}_{3}\right): \delta-13.43(\mathrm{tm}, J=468.9,17.3 \mathrm{~Hz})$.

$v_{\max }\left(\right.$ neat) $/ \mathrm{cm}^{-1}:$ 2976, 2938, 2885, 2424, 2400, 1456, 1393, $1272,1243,1222,1153,1025,957,896$, $862,755,635,600,572,515,450,410$.

HRMS: calcd. for $\mathrm{C}_{14} \mathrm{H}_{28} \mathrm{P}^{+}$: 231.2236 [M-OTf] ${ }^{+}$; found $\left(\mathrm{ESI}^{+}\right)$: 231.2239.

m.p./ ${ }^{\circ} \mathrm{C}: 115-117$. 
Bis-(2,3-dimethyl-2-butyl)phosphonium trifluoromethanesulfonate (1d)

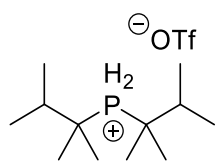

GP2 was performed using 2,3-dimethyl-2-butyl acetate $(0.433 \mathrm{~g}, 3.00 \mathrm{mmol})$. Bis-(2,3-dimethyl-2butyl)phosphonium trifluoromethanesulfonate $1 \mathrm{~d}(0.281 \mathrm{~g}, 0.797 \mathrm{mmol}, 80 \%)$ was obtained as a colourless crystalline solid.

${ }^{1} \mathrm{H}$ NMR $\left(500 \mathrm{MHz}, \mathrm{CDCl}_{3}\right): \delta 6.46(\mathrm{~d}, J=472.2 \mathrm{~Hz}, 2 \mathrm{H}), 2.15(\mathrm{dh}, J=10.8,6.8 \mathrm{~Hz}, 2 \mathrm{H}), 1.52(\mathrm{~d}, J=18.8$, $12 \mathrm{H}), 1.09(\mathrm{~d}, J=6.8,12 \mathrm{H})$.

${ }^{13} \mathrm{C}\left\{{ }^{1} \mathrm{H}\right\}$ NMR $(126 \mathrm{MHz}, \mathrm{CDCl})$ ): $\delta 120.8(\mathrm{q}, J=320.1 \mathrm{~Hz}), 39.8(\mathrm{~d}, J=32.1 \mathrm{~Hz}), 35.4,22.7,17.5(\mathrm{~d}, J=$ $6.8 \mathrm{~Hz})$.

${ }^{19} \mathrm{~F}$ NMR (470 MHz, $\left.\mathrm{CDCl}_{3}\right): \delta-78.37$.

${ }^{31} \mathrm{P}$ NMR (202 MHz, $\left.\mathrm{CDCl}_{3}\right): \delta 8.02(\mathrm{tm}, J=472.3 \mathrm{~Hz})$.

$V_{\max }($ neat $) / \mathrm{cm}^{-1}: 2973,2415,1470,1392,1377,1254,1222,1157,1083,1025,934,757,635,572,516$, 423.

HRMS: calcd. for $\mathrm{C}_{12} \mathrm{H}_{28} \mathrm{P}^{+}: 203.1923$ [M-OTf] ${ }^{+}$; found (ESI $\left.{ }^{+}\right)$: 203.1922.

m.p. $/{ }^{\circ} \mathrm{C}: 151-154$. 
Bis-(2,3,3-trimethyl-2-butyl)phosphonium trifluoromethanesulfonate (1e)

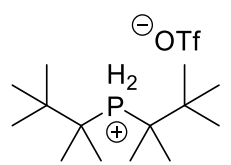

GP2 was performed using 2,3,3-trimethyl-2-butyl acetate $(0.475 \mathrm{~g}, 3.00 \mathrm{mmol})$. Bis-(2,3,3-trimethyl2-butyl)phosphonium trifluoromethanesulfonate 1e $(0.294 \mathrm{~g}, 0.773 \mathrm{mmol}, 77 \%)$ was obtained as a colourless crystalline solid.

A triplet of $13-e t s$ is expected in the ${ }^{31} \mathrm{P}$ NMR spectrum, from ${ }^{1} J_{\mathrm{P}-\mathrm{H}}$ and ${ }^{3} \int_{\mathrm{P}-\mathrm{H}}$ couplings respectively. However, the lower intensity peaks of the multiplet are not observed due to poor signal:noise, and as such is reported below as a triplet of multiplets with both coupling constants given.

${ }^{1} \mathrm{H}$ NMR $\left(500 \mathrm{MHz}, \mathrm{CDCl}_{3}\right): \delta 6.50(\mathrm{~d}, J=468.7 \mathrm{~Hz}, 2 \mathrm{H}), 1.57(\mathrm{~d}, J=18.6 \mathrm{~Hz}, 12 \mathrm{H}), 1.18(\mathrm{~s}, 18 \mathrm{H})$.

${ }^{13} \mathrm{C}\left\{{ }^{1} \mathrm{H}\right\}$ NMR $\left(126 \mathrm{MHz}, \mathrm{CDCl}_{3}\right): \delta 120.8(\mathrm{q}, J=320.1 \mathrm{~Hz}), 43.7(\mathrm{~d}, J=29.5 \mathrm{~Hz}), 38.7,25.8(\mathrm{~d}, J=5.7 \mathrm{~Hz})$, 21.9.

${ }^{19} \mathrm{~F} \mathrm{NMR}\left(470 \mathrm{MHz}, \mathrm{CDCl}_{3}\right): \delta-78.22$.

31P NMR (202 MHz, $\left.\mathrm{CDCl}_{3}\right): \delta-7.25(\mathrm{tm}, J=469.1,18.6 \mathrm{~Hz})$.

$V_{\max }($ neat $) / \mathrm{cm}^{-1}:$ 2981, 2881, 2430, 1476, 1388, 1250, 1224, 1148, 1029, 964, 755, 636, 572, 516.

HRMS: calcd. for $\mathrm{C}_{14} \mathrm{H}_{32} \mathrm{P}^{+}: 231.2236$ [M-OTf] $^{+}$; found $\left.(\mathrm{ESI})^{+}\right): 231.2235$.

m.p. $/{ }^{\circ} \mathrm{C}: 134-137$. 
Di-(2-methyl-4-phenyl-2-butyl)phosphonium trifluoromethanesulfonate (1f)<smiles>CC(C)(CCc1ccccc1)PC(C)(C)CCc1ccccc1</smiles>

Conditions 1: GP2 was performed using 2-methyl-4-phenyl-2-butyl isobutyrate $(0.619 \mathrm{~g}, 3.00 \mathrm{mmol})$. Di-(2-methy-4-phenyl-2-butyl)phosphonium trifluoromethanesulfonate $1 \mathrm{f}(0.398 \mathrm{~g}, 0.835 \mathrm{mmol}, 83 \%)$ was obtained as a colourless crystalline solid.

Conditions 2: GP2 was performed on a $5.0 \mathrm{mmol}$ scale in a $100 \mathrm{~mL}$ CO-ware reactor using $\mathrm{Zn}_{3} \mathrm{P}_{2}(78.0 \%$ purity, $0.827 \mathrm{~g}, 2.50 \mathrm{mmol})$, 2-methyl-4-phenyl-2-butyl isobutyrate (3.51 g, $15.0 \mathrm{mmol})$, trimethylsilyl trifluoromethanesulfonate $(0.905 \mathrm{~mL}, 5.00 \mathrm{mmol})$ and degassed hydrochloric acid $(5.0 \mathrm{M} ; 10 \mathrm{~mL}, 50$ mmol). Di-(2-methyl-4-phenyl-2-butyl)phosphonium trifluoromethanesulfonate 1 f $(2.02 \mathrm{~g}, 4.24$ $\mathrm{mmol}, 85 \%$ ) was obtained as a colourless crystalline solid.

${ }^{1} \mathrm{H}$ NMR $\left(500 \mathrm{MHz}, \mathrm{CDCl}_{3}\right): \delta$ 7.30-7.26 (m, 4H), 7.24-7.19 (m, 2H), 7.18-7.14 (m, 4H), $6.57(\mathrm{~d}, J=476.5$ $\mathrm{Hz}, 2 \mathrm{H}), 2.76-2.69(\mathrm{~m}, 4 \mathrm{H}), 2.14-2.05(\mathrm{~m}, 4 \mathrm{H}), 1.60(\mathrm{~d}, \mathrm{~J}=18.4 \mathrm{~Hz}, 12 \mathrm{H})$.

${ }^{13} \mathrm{C}\left\{{ }^{1} \mathrm{H}\right\}$ NMR (126 MHz, CDCl 3$): \delta 139.7,129.0,128.5,126.8,120.7(\mathrm{q}, J=319.9 \mathrm{~Hz}), 41.8,35.4(\mathrm{~d}, J=$ $33.1 \mathrm{~Hz}), 29.8(\mathrm{~d}, J=8.1 \mathrm{~Hz}), 25.2$.

${ }^{19}$ F NMR (470 MHz, $\left.\mathrm{CDCl}_{3}\right): \delta-78.34$.

${ }^{31} \mathrm{P}$ NMR (202 MHz, $\left.\mathrm{CDCl}_{3}\right): \delta 14.52(\mathrm{tm}, J=476.6 \mathrm{~Hz})$.

$v_{\text {max }}($ neat $) / \mathrm{cm}^{-1}: 3065,3029,2965,2936,2863,2427,2397,1603,1498,1456,1379,1273,1248,1220$, 1152, 1025, 986, 935, 745, 701, 636, 572, 516, 466, 418.

HRMS: calcd. for $\mathrm{C}_{22} \mathrm{H}_{32} \mathrm{P}^{+}$: 327.2236 [M-OTf] ${ }^{+}$; found (ESI ${ }^{+}$): 327.2239 . 
Di-(2-Methyl-4-(4-bromophenyl)-2-butyl)phosphonium trifluoromethanesulfonate (1g)<smiles>CC(C)(CCc1ccc(Br)cc1)PC(C)(C)CCc1ccc(Br)cc1</smiles>

GP2 was performed using 2-methyl-4-(4-bromophenyl)-2-butyl acetate (0.856 g, $3.00 \mathrm{mmol})$. Di-(2methyl-4-(4-bromophenyl)-2-butyl)phosphonium trifluoromethanesulfonate $1 \mathrm{~g}(0.401 \mathrm{~g}, 0.632 \mathrm{mmol}$, $63 \%)$ was obtained as a colourless crystalline solid.

${ }^{1} \mathrm{H}$ NMR (500 MHz, CDCl $)$ ): $\delta 7.39(\mathrm{~d}, J=7.9 \mathrm{~Hz}, 4 \mathrm{H}), 7.05(\mathrm{~d}, J=7.9 \mathrm{~Hz}, 4 \mathrm{H}), 6.64(\mathrm{~d}, J=478.1 \mathrm{~Hz}, 2 \mathrm{H})$, 2.71-2.64 (m, 4H), 2.10-2.00 (m, 4H), 1.62-1.52 (d, J = 18.2 Hz, $12 \mathrm{H})$.

${ }^{13} \mathrm{C}\left\{{ }^{1} \mathrm{H}\right\}$ NMR (126 MHz, CDCl 3$): \delta 138.8,132.0,130.3,120.7(\mathrm{q}, J=320.1 \mathrm{~Hz}), 120.6,41.9,35.2(\mathrm{~d}, J=$ $31.9 \mathrm{~Hz}), 29.2(\mathrm{~d}, J=8.0 \mathrm{~Hz}), 25.2$.

${ }^{19} \mathrm{~F} \mathrm{NMR}\left(470 \mathrm{MHz}, \mathrm{CDCl}_{3}\right): \delta-78.36$.

${ }^{31}$ P NMR (202 MHz, $\left.\mathrm{CDCl}_{3}\right): \delta 14.43(\mathrm{tm}, J=479.6 \mathrm{~Hz})$.

$v_{\max }\left(\right.$ neat) $/ \mathrm{cm}^{-1}: 2965,2943,2873,2416,2391,1488,1471,1402,1377,1252,1222,1154,1071,1027$, 1009, 922, 798, 756, 636, 608, 572, 515, 486, 456.

HRMS: calcd. for $\mathrm{C}_{22} \mathrm{H}_{30} \mathrm{Br}_{2} \mathrm{P}^{+}$: 483.0446 [M-OTf] ${ }^{+}$; found $\left.(\mathrm{ESI})^{+}\right)$: 483.0448.

m.p./ ${ }^{\circ} \mathrm{C}: 93-95$. 


\section{Di-(1-methylcyclopentyl)phosphonium trifluoromethanesulfonate (1h)}

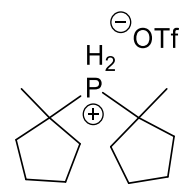

Conditions 1: GP2 was performed using 1-methylcyclopentyl acetate $(0.427 \mathrm{~g}, 3.00 \mathrm{mmol})$. Di-(1methylcyclopentyl)phosphonium trifluoromethanesulfonate $1 \mathrm{~h}(0.118 \mathrm{~g}, 0.339 \mathrm{mmol}, 34 \%)$ was obtained as a colourless crystalline solid.

Conditions 2: GP2 was performed using 1-methylcyclopentyl acetate $(0.853 \mathrm{~g}, 6.00 \mathrm{mmol})$. Di-(1methylcyclopentyl)phosphonium trifluoromethanesulfonate $\mathbf{1 h}(0.193 \mathrm{~g}, 0.554 \mathrm{mmol}, 55 \%)$ was obtained as a colourless crystalline solid.

${ }^{1} \mathrm{H}$ NMR (500 MHz, CDCl $): \delta 6.53(\mathrm{~d}, J=478.1 \mathrm{~Hz}, 2 \mathrm{H}), 2.21-2.08(\mathrm{~m}, 4 \mathrm{H}), 1.95-1.82(\mathrm{~m}, 12 \mathrm{H}), 1.50(\mathrm{~d}$, $J=18.0 \mathrm{~Hz}, 6 \mathrm{H})$.

${ }^{13} \mathrm{C}\left\{{ }^{1} \mathrm{H}\right\}$ NMR (126 MHz, CDCl 3$): \delta 120.7(\mathrm{q}, J=320.0 \mathrm{~Hz}), 38.6(\mathrm{~d}, J=2.7 \mathrm{~Hz}), 37.3(\mathrm{~d}, J=38.7 \mathrm{~Hz}), 24.4$ (d, $J=9.1 \mathrm{~Hz}), 23.7(\mathrm{~d}, J=3.4 \mathrm{~Hz})$.

${ }^{19} \mathrm{~F} \mathrm{NMR}\left(470 \mathrm{MHz}, \mathrm{CDCl}_{3}\right): \delta-78.50$.

${ }^{31} \mathrm{P}$ NMR (202 MHz, $\left.\mathrm{CDCl}_{3}\right): \delta 18.22(\mathrm{tm}, J=478.2 \mathrm{~Hz})$.

$v_{\max }($ neat $) / \mathrm{cm}^{-1}: 2969$ 2878, 2442, 2415, 1453, 1390, 1248, 1222, 1147, 1025, 943, 915, 755, 635, 572, $515,475.40$.

HRMS: calcd. for $\mathrm{C}_{12} \mathrm{H}_{24} \mathrm{P}^{+}$: 199.1610 [M-OTf] $^{+}$; found $\left(\mathrm{ESI}^{+}\right)$: 199.1619.

m.p. $/{ }^{\circ} \mathrm{C}: 58-61$. 
Di-(1-methylcyclohexyl)phosphonium trifluoromethanesulfonate (1i)

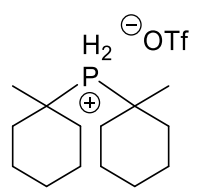

GP2 was performed using 1-methylcyclohexyl acetate $(0.469 \mathrm{~g}, 3.00 \mathrm{mmol})$. Di-(1methylcyclohexyl)phosphonium trifluoromethanesulfonate 1 i $(0.278 \mathrm{~g}, 0.739 \mathrm{mmol}, 74 \%)$ was obtained as a colourless crystalline solid.

${ }^{1} \mathrm{H}$ NMR $\left(500 \mathrm{MHz}, \mathrm{CDCl}_{3}\right): \delta 6.33(\mathrm{~d}, J=469.5 \mathrm{~Hz}, 2 \mathrm{H}), 2.05-1.96(\mathrm{~m}, 4 \mathrm{H}), 1.89-1.82(\mathrm{~m}, 4 \mathrm{H}), 1.69-1.60$ (m, 16H), 1.45-1.41 (m, 2H).

${ }^{13} \mathrm{C}\left\{{ }^{1} \mathrm{H}\right\}$ NMR (126 MHz, CDCl 3$): \delta 120.7(\mathrm{q}, J=320.1 \mathrm{~Hz}), 36.2(\mathrm{~d}, J=33.8 \mathrm{~Hz}), 35.1,24.8,21.6 .20 .7(\mathrm{~d}$, $J=9.6 \mathrm{~Hz})$.

${ }^{19} \mathrm{~F} \mathrm{NMR}\left(470 \mathrm{MHz}, \mathrm{CDCl}_{3}\right): \delta-78.41$.

${ }^{31}$ P NMR (202 MHz, $\left.\mathrm{CDCl}_{3}\right): \delta 17.44(\mathrm{tm}, J=470.1 \mathrm{~Hz})$.

$v_{\max }($ neat $) / \mathrm{cm}^{-1}: 2932,2862,2426,2403,1460,1388,1350,1250,1222,1151,1025,988,968,941$, $920,847,756,635,613,571,515,466,440$.

HRMS: calcd. for $\mathrm{C}_{14} \mathrm{H}_{28} \mathrm{P}^{+}$: 227.1923 [M-OTf] ${ }^{+}$; found $\left(\mathrm{ESI}^{+}\right)$: 227.1928.

m.p./ ${ }^{\circ} \mathrm{C}: 138-142$. 


\section{Di-(1-phenylcyclohexyl)phosphonium trifluoromethanesulfonate (1j)}

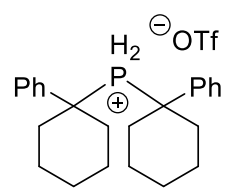

Conditions 1: GP2 was performed using a solution of 1-phenylcyclohexyl acetate $(0.655 \mathrm{~g}, 3.00 \mathrm{mmol})$ dissolved in degassed anhydrous dichloromethane $(2.0 \mathrm{~mL})$, with chamber two heated to $40{ }^{\circ} \mathrm{C}$. Di-(1phenylcyclohexyl)phosphonium trifluoromethanesulfonate $\mathbf{1 j}(0.203 \mathrm{~g}, 0.369 \mathrm{mmol}, 37 \%)$ was obtained as a colourless crystalline solid.

Conditions 2: GP2 was performed using 1-phenylcyclohexyl acetate $(0.655 \mathrm{~g}, 3.00 \mathrm{mmol})$, with chamber two heated to $40{ }^{\circ} \mathrm{C}$. Di-(1-phenylcyclohexyl)phosphonium trifluoromethanesulfonate $\mathbf{1 j}$ (0.291 g, $0.581 \mathrm{mmol}, 58 \%)$ was obtained as a colourless crystalline solid.

${ }^{1} \mathrm{H}$ NMR $\left(500 \mathrm{MHz}, \mathrm{CDCl}_{3}\right): \delta$ 7.50-7.44 (m, 4H), 7.43-7.32 (m, 7H, Ar-H + one peak of $\mathrm{PH}_{2}$ doublet), 6.18 (app. $\mathrm{s}, 1 \mathrm{H}$, one peak of $\mathrm{PH}_{2}$ doublet), 2.10-2.00 (m, 4H), 1.89-1.78 (m, $\left.4 \mathrm{H}\right), 1.53-1.41(\mathrm{~m}, 6 \mathrm{H})$, $1.38-1.23(\mathrm{~m}, 6 \mathrm{H})$.

${ }^{13} \mathrm{C}\left\{{ }^{1} \mathrm{H}\right\}$ NMR (126 MHz, CDCl 3$): \delta 133.6,130.0$ (d, J = 2.8 Hz), $129.2(\mathrm{~d}, J=3.5 \mathrm{~Hz}), 128.0(\mathrm{~d}, J=5.4 \mathrm{~Hz})$, $120.9(q, J=320.2 \mathrm{~Hz}), 42.3(\mathrm{~d}, J=31.9 \mathrm{~Hz}), 33.1,25.0,21.0$ (d, $J=9.7 \mathrm{~Hz})$.

${ }^{19} \mathrm{~F} \mathrm{NMR}\left(470 \mathrm{MHz}, \mathrm{CDCl}_{3}\right): \delta-78.26$.

${ }^{31} \mathrm{P}$ NMR (202 MHz, $\left.\mathrm{CDCl}_{3}\right): \delta 18.28(\mathrm{tm}, J=478.6 \mathrm{~Hz})$.

$v_{\text {max }}($ neat $) / \mathrm{cm}^{-1}: 2940,2858,2398,2366,1448,1256,1222,1142,1051,1026,948,750,699,634,571$, 514,415 .

HRMS: calcd. for $\mathrm{C}_{24} \mathrm{H}_{32} \mathrm{P}^{+}$: 351.2236 [M-OTf] $^{+}$; found $\left(\mathrm{ESI}^{+}\right)$: 351.2232.

m.p./ ${ }^{\circ} \mathrm{C}: 167-170$. 


\section{Di-(1-Adamantyl)phosphonium trifluoromethanesulfonate (1k)}

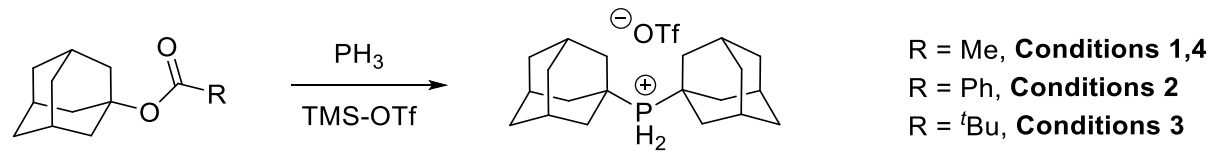

Conditions 1: GP2 was performed using a solution of 1-adamantyl acetate $(0.583 \mathrm{~g}, 3.00 \mathrm{mmol})$ dissolved in degassed anhydrous dichloromethane $(2.0 \mathrm{~mL})$, with chamber two heated to $40^{\circ} \mathrm{C}$. Di- $(1-$ adamantyl)phosphonium trifluoromethanesulfonate $1 \mathbf{k}(0.378 \mathrm{~g}, 0.835 \mathrm{mmol}, 84 \%)$ was obtained as a colourless crystalline solid.

Conditions 2: GP2 was performed using a solution of 1-adamantyl benzoate $(0.769 \mathrm{~g}, 3.00 \mathrm{mmol})$ dissolved in degassed anhydrous dichloromethane $(2.0 \mathrm{~mL})$, with chamber two heated to $40^{\circ} \mathrm{C}$. Di-(1adamantyl)phosphonium trifluoromethanesulfonate $1 \mathbf{k}(0.373 \mathrm{~g}, 0.824 \mathrm{mmol}, 82 \%)$ was obtained as a colourless crystalline solid.

Conditions 3: GP2 was performed using a solution of 1-adamantyl pivalate $(0.709 \mathrm{~g}, 3.00 \mathrm{mmol})$ dissolved in degassed anhydrous dichloromethane $(2.0 \mathrm{~mL})$, with chamber two heated to $40^{\circ} \mathrm{C}$. Di-(1adamantyl)phosphonium trifluoromethanesulfonate $1 \mathbf{k}(0.380 \mathrm{~g}, 0.840 \mathrm{mmol}, 84 \%)$ was obtained as a colourless crystalline solid.

Conditions 4: GP2 was performed on a $5.0 \mathrm{mmol}$ scale in a $100 \mathrm{~mL}$ CO-ware reactor using $\mathrm{Zn}_{3} \mathrm{P}_{2}$ (78.0\% purity, $0.827 \mathrm{~g}, 2.50 \mathrm{mmol})$, 1-adamantyl acetate $(2.91 \mathrm{~g}, 15.0 \mathrm{mmol})$, trimethylsilyl trifluoromethanesulfonate $(0.905 \mathrm{~mL}, 5.00 \mathrm{mmol})$ and degassed hydrochloric acid $(5.0 \mathrm{M} ; 10 \mathrm{~mL}, 50$ mmol). Di-(1-adamantyl)phosphonium trifluoromethanesulfonate $1 \mathrm{k}$ (2.06 g, $4.55 \mathrm{mmol}, 91 \%)$ was obtained as a colourless crystalline solid.

Note: one of the peaks of the doublet at $35.4 \mathrm{ppm}$ in the ${ }^{13} \mathrm{C}\left\{{ }^{1} \mathrm{H}\right\}$ NMR spectrum overlaps with the doublet at $35.5 \mathrm{ppm}$, but can be observed with additional processing.

${ }^{1} \mathrm{H}$ NMR (500 MHz, $\mathrm{CDCl}_{3}$ ): $\delta 5.99$ (d, $J=469.4 \mathrm{~Hz}, 2 \mathrm{H}$ ), 2.21-2.16 (br. m, 12H), 2.14-2.09 (br. m, 6H), 1.84-1.78 (br. m, 12H).

${ }^{13} \mathrm{C}\left\{{ }^{1} \mathrm{H}\right\}$ NMR (126 MHz, CDCl 3$): \delta 120.8(q, J=320.4 \mathrm{~Hz}), 38.9(\mathrm{~d}, J=2.0 \mathrm{~Hz}), 35.5(\mathrm{~d}, J=2.0 \mathrm{~Hz}), 35.4$ $(\mathrm{d}, J=34.8 \mathrm{~Hz}), 27.5(\mathrm{~d}, J=10.4 \mathrm{~Hz})$.

${ }^{19} \mathrm{~F}$ NMR (470 MHz, $\left.\mathrm{CDCl}_{3}\right): \delta-78.31$.

${ }^{31}$ P NMR (202 MHz, CDCl $): \delta 13.04(t, J=469.6 \mathrm{~Hz})$. 
$V_{\max }($ neat $) / \mathrm{cm}^{-1}:$ 2906, 2854, 2424, 2400, 1450, 1345, 1305, 1276, 1245, 1221, 1154, 1022, 973, 944 , $898,755,634,571,515,489,447.40,417$.

HRMS: calcd. for $\mathrm{C}_{20} \mathrm{H}_{32} \mathrm{P}^{+}: 303.2236$ [M-OTf] $]^{+}$; found $\left(\mathrm{ESI}^{+}\right)$: 303.2258.

m.p. $/{ }^{\circ} \mathrm{C}:>280$, decomposition. 
Di-(2-methyl-2-adamantyl)phosphonium trifluoromethanesulfonate (1I)

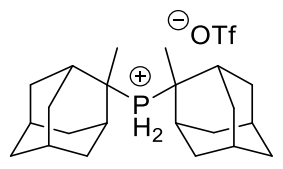

GP2 was performed using 2-methyl-2-adamantyl acetate $(0.625 \mathrm{~g}, 3.00 \mathrm{mmol})$. Di-(2-methyl-2adamantyl)phosphonium trifluoromethanesulfonate $\mathbf{1 l}(0.145 \mathrm{~g}, 0.302 \mathrm{mmol}, 30 \%)$ was obtained as a colourless crystalline solid.

${ }^{1} \mathrm{H}$ NMR $\left(500 \mathrm{MHz}, \mathrm{CDCl}_{3}\right): \delta 6.82(\mathrm{~d}, J=466.1 \mathrm{~Hz}, 2 \mathrm{H}), 2.29-2.19(\mathrm{~m}, 12 \mathrm{H}), 2.16-2.11(\mathrm{~m}, 2 \mathrm{H}), 1.99-$ $1.90(\mathrm{~m}, 6 \mathrm{H}), 1.80-1.68(\mathrm{~m}, 14 \mathrm{H})$.

${ }^{13} \mathrm{C}\left\{{ }^{1} \mathrm{H}\right\}$ NMR (126 MHz, CDCl 3 ): $\delta 121.9(\mathrm{q}, J=320.2 \mathrm{~Hz}), 46.0(\mathrm{~d}, J=30.1 \mathrm{~Hz}), 38.6,35.7(\mathrm{~d}, J=1.7 \mathrm{~Hz})$, $33.8(\mathrm{~d}, J=3.8 \mathrm{~Hz}), 31.5(\mathrm{~d}, J=8.7 \mathrm{~Hz}), 26.9,26.8,22.2(\mathrm{~d}, J=2.5 \mathrm{~Hz})$.

${ }^{19}$ F NMR (470 MHz, $\left.\mathrm{CDCl}_{3}\right): \delta-78.36$.

${ }^{31}$ P NMR (202 MHz, $\left.\mathrm{CDCl}_{3}\right): \delta-4.30(\mathrm{tm}, J=466.2 \mathrm{~Hz})$.

$v_{\max }($ neat $) / \mathrm{cm}^{-1}: 2912,2869,2449,2408,1462,1282,1245,1223,1150,1026,955,934,755,635,572$, 516.

HRMS: calcd. for $\mathrm{C}_{22} \mathrm{H}_{36} \mathrm{P}^{+}: 331.2549$ [M-OTf] $]^{+}$; found $\left(\mathrm{ESI}^{+}\right)$: 351.2553 .

m.p./ ${ }^{\circ} \mathrm{C}: 202-206$. 


\section{Dicedrylphosphonium trifluoromethanesulfonate $(1 \mathrm{~m})$}

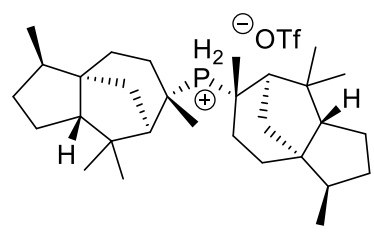

GP2 was performed using (+)-cedryl acetate $(0.793 \mathrm{~g}, 3.00 \mathrm{mmol})$ with chamber two heated to $40{ }^{\circ} \mathrm{C}$. Dicedrylphosphonium trifluoromethanesulfonate $1 \mathrm{~m}(0.419 \mathrm{~g}, 0.252 \mathrm{mmol}, 25 \%)$ was obtained as a colourless crystalline solid.

${ }^{1} \mathrm{H}$ NMR $\left(500 \mathrm{MHz}, \mathrm{CDCl}_{3}\right): \delta 6.52(\mathrm{~d}, J=465.1 \mathrm{~Hz}, 2 \mathrm{H}), 2.42-2.32(\mathrm{~m}, 2 \mathrm{H}), 2.14-1.86(\mathrm{~m}, 8 \mathrm{H}), 1.85-1.73$ $(\mathrm{m}, 12 \mathrm{H}), 1.63-1.54(\mathrm{~m}, 4 \mathrm{H}), 1.48-1.39(\mathrm{~m}, 4 \mathrm{H}), 1.38-1.25(\mathrm{~m}, 8 \mathrm{H}), 1.10(\mathrm{~s}, 6 \mathrm{H}), 0.89(\mathrm{~d}, J=7.1 \mathrm{~Hz}, 6 \mathrm{H})$. ${ }^{13} \mathrm{C}\left\{{ }^{1} \mathrm{H}\right\}$ NMR (126 MHz, CDCl $): \delta 120.7$ (q, $\left.J=320.5 \mathrm{~Hz}\right), 57.8,55.2,54.7,47.4(\mathrm{~d}, J=13.0 \mathrm{~Hz}), 46.2$ (d, $J=25.4 \mathrm{~Hz}$ ), $43.2(\mathrm{~d}, J=6.3 \mathrm{~Hz}), 41.5,36.4,34.0,30.5,29.7(\mathrm{~d}, J=2.1 \mathrm{~Hz}), 29.5,27.0,25.3,15.4$.

${ }^{19}$ F NMR (470 MHz, $\left.\mathrm{CDCl}_{3}\right): \delta-78.26$.

${ }^{31}$ P NMR (202 MHz, $\left.\mathrm{CDCl}_{3}\right): \delta 11.18(\mathrm{tm}, J=466.5 \mathrm{~Hz})$.

$V_{\max }($ neat $) / \mathrm{cm}^{-1}: 2941,2871,2418,1464,1392,1281,1249,1225,1149,1029,972,942,755,636,571$, 516.

HRMS: calcd. for $\mathrm{C}_{30} \mathrm{H}_{52} \mathrm{P}^{+}: 443.3801$ [M-OTf] $^{+}$; found $\left(\mathrm{ESI}^{+}\right)$: 443.3803.

m.p./ ${ }^{\circ} \mathrm{C}: 142-144$. 


\section{Tribenzhydrylphosphonium trifluoromethanesulfonate (1n)}

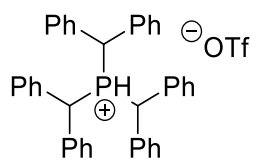

GP2 was performed using a solution of benzhydryl acetate ${ }^{18}(0.679 \mathrm{~g}, 3.00 \mathrm{mmol})$ dissolved in degassed anhydrous dichloromethane $(2.0 \mathrm{~mL})$, with chamber 2 heated to $40{ }^{\circ} \mathrm{C}$. Tribenzhydrylphosphonium trifluoromethanesulfonate $1 \mathrm{n}(0.516 \mathrm{~g}, 0.756 \mathrm{mmol}, 76 \%)$ was obtained as a colourless crystalline solid.

The PH signal in the ${ }^{1} \mathrm{H}$ NMR spectrum (8.82 ppm) appears as a broad doublet of quartets (from coupling to the ${ }^{31} \mathrm{P}$ and benzhydrylic methine protons, respectively), but the resolution is not sufficient to allow accurate measurement of the second coupling constant.

${ }^{1}{ }^{H}$ NMR $\left(500 \mathrm{MHz}, \mathrm{CDCl}_{3}\right): \delta 8.82(\mathrm{dm}, J=486.8 \mathrm{~Hz}, 1 \mathrm{H}) 7.09-7.22(\mathrm{~m}, 30 \mathrm{H}), 5.13(\mathrm{dd}, J=17.4,7.4 \mathrm{~Hz}$, $3 \mathrm{H})$.

${ }^{13} \mathrm{C}\left\{{ }^{1} \mathrm{H}\right\}$ NMR (126 MHz, CDCl 3$): \delta 132.8(\mathrm{~d}, J=4.0 \mathrm{~Hz}), 129.6,129.1(\mathrm{~d}, J=6.9 \mathrm{~Hz}), 128.5,121.3(\mathrm{q}, J=$ $320.6 \mathrm{~Hz}), 46.1(\mathrm{~d}, J=34.2 \mathrm{~Hz})$.

${ }^{19}$ F NMR (376 MHz, $\left.\mathrm{CDCl}_{3}\right): \delta-77.89$.

${ }^{31}$ P NMR (162 MHz, $\left.\mathrm{CDCl}_{3}\right): \delta 20.05$ (dq $\left.J=488.9,17.3 \mathrm{~Hz}\right)$.

$v_{\max }($ neat $) / \mathrm{cm}^{-1}:$ 3064, 3033, 2910, 2420, 1600, 1586, 1494, 1453, 1255, 1224, 1153, 1029, 929, 744, $693,637,572,511,475$.

HRMS: calcd. for $\mathrm{C}_{12} \mathrm{H}_{28} \mathrm{P}^{+}$: 203.1923 [M-OTf] ${ }^{+}$; found (ESI ${ }^{+}$): 203.1920.

m.p./ ${ }^{\circ} \mathrm{C}: 215-218$. 


\section{Alkylation of $\mathrm{PH}_{3}$ with tert-Butyl Acetate (Manuscript Scheme 4A)}

Di-tert-butylphosphonium trifluoromethanesulfonate (10),

tert-butyl(2,4,4-trimethyl-2-pentyl)phosphonium trifluoromethanesulfonate (1p) and bis-(2,4,4-trimethyl-2-pentyl)phosphonium trifluoromethanesulfonate (1q)

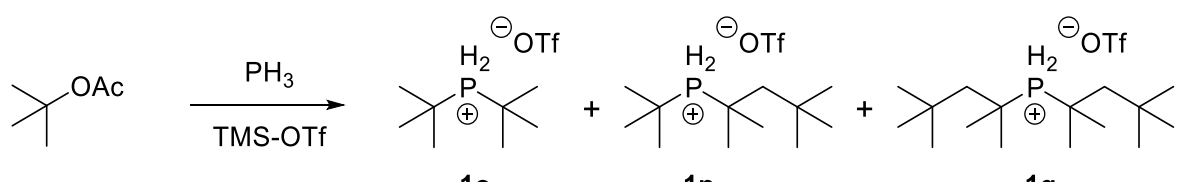

10

$1 p$

$1 \mathrm{q}$

GP2 was performed using tert-butyl acetate $(0.80 \mathrm{~mL}, 6.00 \mathrm{mmol})$. The product distribution was determined by ${ }^{31}$ P NMR spectroscopic analysis of the reaction mixture (1.0:3.6:5.1 10:1p:1q) prior to work-up. Work-up as per GP2 afforded a mixture of 1o, 1p and 1q (0.115 g, 0.32:0.42:0.26 10:1p:1q) as a colourless crystalline solid. Assignments were confirmed by comparison to literature data (10) $)^{19}$ or by independent synthesis of pure samples (1q, vide infra).

Characterisation data for 10

${ }^{1}$ H NMR (400 MHz, $\left.\mathrm{CDCl}_{3}\right): \delta 6.45(\mathrm{~d}, J=474.8 \mathrm{~Hz}, 2 \mathrm{H}), 1.58(\mathrm{~d}, J=18.0 \mathrm{~Hz}, 18 \mathrm{H})$.

${ }^{13} \mathrm{C}\left\{{ }^{1} \mathrm{H}\right\}$ NMR (126 MHz, $\left.\mathrm{CDCl}\right): \delta 120.6(\mathrm{q}, J=319.9 \mathrm{~Hz}), 31.3(\mathrm{~d}, J=29.5 \mathrm{~Hz}), 28.0(\mathrm{~d}, J=1.8 \mathrm{~Hz})$.

${ }^{31} \mathrm{P}\left\{{ }^{1} \mathrm{H}\right\}$ NMR (202 MHz, $\left.\mathrm{CDCl}_{3}\right): \delta 22.30$.

Characterisation data for $\mathbf{1 p}$

${ }^{1} \mathrm{H}$ NMR (400 MHz, CDCl 3$): \delta 6.38(\mathrm{~d}, J=472.5 \mathrm{~Hz}, 2 \mathrm{H}), 1.84(\mathrm{~d}, J=12.6 \mathrm{~Hz}, 2 \mathrm{H}), 1.72(\mathrm{~d}, J=20.4 \mathrm{~Hz}$, $6 \mathrm{H}), 1.59(\mathrm{~d}, \mathrm{~J}=17.7 \mathrm{~Hz}, 9 \mathrm{H}), 1.10(\mathrm{~s}, 9 \mathrm{H})$.

${ }^{13} \mathrm{C}\left\{{ }^{1} \mathrm{H}\right\}$ NMR (126 MHz, $\left.\mathrm{CDCl}_{3}\right): \delta 120.6(\mathrm{q}, J=319.9 \mathrm{~Hz}), 50.3,37.5(\mathrm{~d}, J=31.9 \mathrm{~Hz}), 34.1(\mathrm{~d}, J=12.5$ $\mathrm{Hz}), 32.2(\mathrm{~d}, J=32.8 \mathrm{~Hz}), 31.8,27.5(\mathrm{~d}, J=2.1 \mathrm{~Hz}), 25.8$.

${ }^{31} \mathrm{P}\left\{{ }^{1} \mathrm{H}\right\}$ NMR (202 MHz, $\left.\mathrm{CDCl}_{3}\right): \delta 25.82$. 
Characterisation data for $\mathbf{1 q}$

${ }^{1} \mathrm{H}$ NMR $\left(400 \mathrm{MHz}, \mathrm{CDCl}_{3}\right): \delta 6.30(\mathrm{~d}, J=470.1 \mathrm{~Hz}, 2 \mathrm{H}), 1.86(\mathrm{~d}, J=12.0 \mathrm{~Hz}, 4 \mathrm{H}), 1.73(\mathrm{~d}, J=20.4 \mathrm{~Hz}$, $12 \mathrm{H}), 1.10(\mathrm{~s}, 18 \mathrm{H})$.

${ }^{13} \mathrm{C}\left\{{ }^{1} \mathrm{H}\right\}$ NMR (126 MHz, $\left.\mathrm{CDCl}_{3}\right): \delta 120.6(\mathrm{q}, J=319.9 \mathrm{~Hz}), 50.5,38.7(\mathrm{~d}, J=29.5 \mathrm{~Hz}), 34.2(\mathrm{~d}, J=12.6$ $\mathrm{Hz}), 31.9,26.2$.

${ }^{31} \mathrm{P}\left\{{ }^{1} \mathrm{H}\right\} \mathrm{NMR}\left(202 \mathrm{MHz}, \mathrm{CDCl}_{3}\right): \delta 29.56$. 
Bis-(2,4,4-trimethyl-2-pentyl)phosphonium trifluoromethanesulfonate (1q)

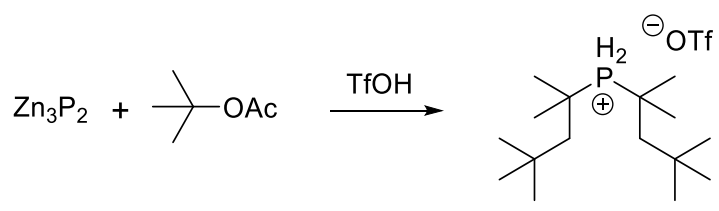

Trifluoromethanesulfonic acid $(0.26 \mathrm{~mL}, 3.00 \mathrm{mmol})$ was added to a mixture of $\mathrm{Zn}_{3} \mathrm{P}_{2}(0.129 \mathrm{~g}, 0.39$ $\mathrm{mmol})$ and anhydrous, degassed tert-butyl acetate $(2.5 \mathrm{~mL})$ at r.t. The resulting heavy black suspension was stirred overnight. The mixture was filtered through a plug of cotton wool, eluting with $\mathrm{Et}_{2} \mathrm{O}$, and the resulting biphasic mixture was placed in a freezer at $-25^{\circ} \mathrm{C}$ overnight. The solids were collected by Büchner filtration, washed with $\mathrm{Et}_{2} \mathrm{O}$ and dried under a flow of air to give di-(2,2,4-trimethyl-2pentyl)phosphonium trifluoromethanesulfonate 1q (30.4 mg, $74.4 \mu \mathrm{mol}, 9 \%)$.

Note that the trifluoromethyl carbon is not observed in the ${ }^{13} \mathrm{C}$ NMR spectrum at this concentration due to low signal:noise, but the presence of the trifluoromethanesulfonate counterion is confirmed by the ${ }^{19} \mathrm{~F}$ NMR and IR spectroscopy and IR .

${ }^{1} \mathrm{H}$ NMR $\left(500 \mathrm{MHz}, \mathrm{CDCl}_{3}\right): \delta 6.31(\mathrm{~d}, J=470.2 \mathrm{~Hz}, 2 \mathrm{H}), 1.86(\mathrm{~d}, J=12.0 \mathrm{~Hz}, 4 \mathrm{H}), 1.73(\mathrm{~d}, J=20.1 \mathrm{~Hz}$, $12 \mathrm{H}), 1.10(\mathrm{~s}, 18 \mathrm{H})$.

${ }^{13} \mathrm{C}\left\{{ }^{1} \mathrm{H}\right\}$ NMR (126 MHz, $\left.\mathrm{CDCl}_{3}\right): \delta 50.7,38.8(\mathrm{~d}, J=29.1 \mathrm{~Hz}), 34.3(\mathrm{~d}, J=12.8 \mathrm{~Hz}), 32.0,26.4$.

${ }^{19} \mathrm{~F}$ NMR (470 MHz, $\left.\mathrm{CDCl}_{3}\right): \delta-78.31$.

${ }^{31}$ P NMR (202 MHz, CDCl $): \delta 29.53(\mathrm{tm}, J=470.0 \mathrm{~Hz})$.

$v_{\max }($ neat $) / \mathrm{cm}^{-1}: 2955,2910,2875,2414,1472,1399,1378,1255,1223,1158,1028,950,756,638$, $572,517$.

HRMS: calcd. for $\mathrm{C}_{16} \mathrm{H}_{36} \mathrm{P}^{+}$: 259.2549 [M-OTf] ${ }^{+}$; found (ESI ${ }^{+}$): 259.2533.

m.p./ ${ }^{\circ} \mathrm{C}: 119-121$. 


\section{Isotope Exchange Studies (Manuscript Scheme 4B)}

\section{Preparation of $5.0 \mathrm{M} \mathrm{DCl}$ in $\mathrm{D}_{2} \mathrm{O}$}

Trimethylsilyl chloride $(6.35 \mathrm{~mL}, 50.0 \mathrm{mmol})$ was added slowly to $\mathrm{D}_{2} \mathrm{O}(10.0 \mathrm{~mL})$, and the mixture was stirred for $1 \mathrm{~h}$. The resulting biphasic mixture was degassed by sparging with nitrogen, and the aqueous phase was removed by syringe for use in isotope exchange studies.

Reaction of 2-methyl-4-phenylbut-2-yl acetate $\left(d_{0}-2\right)$ with $\mathrm{PD}_{3}$

GP2 was performed using 2-methyl-4-phenylbut-2-yl acetate $\boldsymbol{d}_{\mathbf{0}}-\mathbf{2}(0.619 \mathrm{~g}, 3.00 \mathrm{mmol})$ and DCl $(5.0$ $\mathrm{M}$ in $\mathrm{D}_{2} \mathrm{O}$ ). Di-(2-methyl-4-phenylbut-2-yl)phosphonium trifluoromethanesulfonate $\boldsymbol{d}_{\mathbf{n}} \mathbf{- 1 f}(0.245 \mathrm{~g})$ was obtained as a colourless crystalline solid. The isotopic distribution of $\boldsymbol{d}_{\mathbf{n}}-\mathbf{1 f}$ prepared in this way was determined by ESI-MS (Figure S2) and ${ }^{1}$ H NMR spectroscopy (Figure S3). 


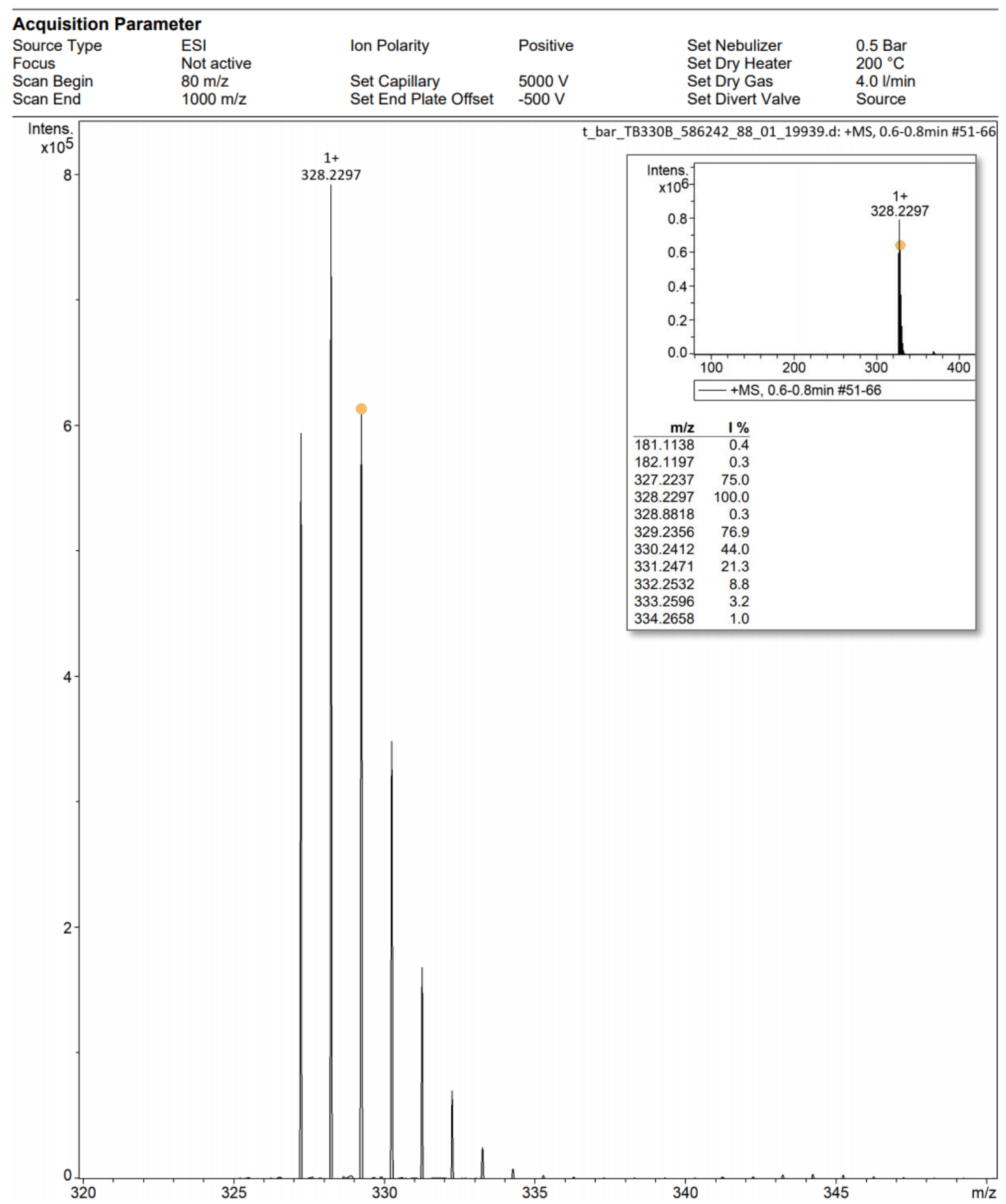

Figure S2. ESI-MS mass distribution for the $\boldsymbol{d}_{\mathbf{n}}-\mathbf{1} \mathbf{f}$ cation prepared from the reaction of $\boldsymbol{d}_{\mathbf{0}} \mathbf{- 2}$ with $\mathrm{PD}_{3}$. 


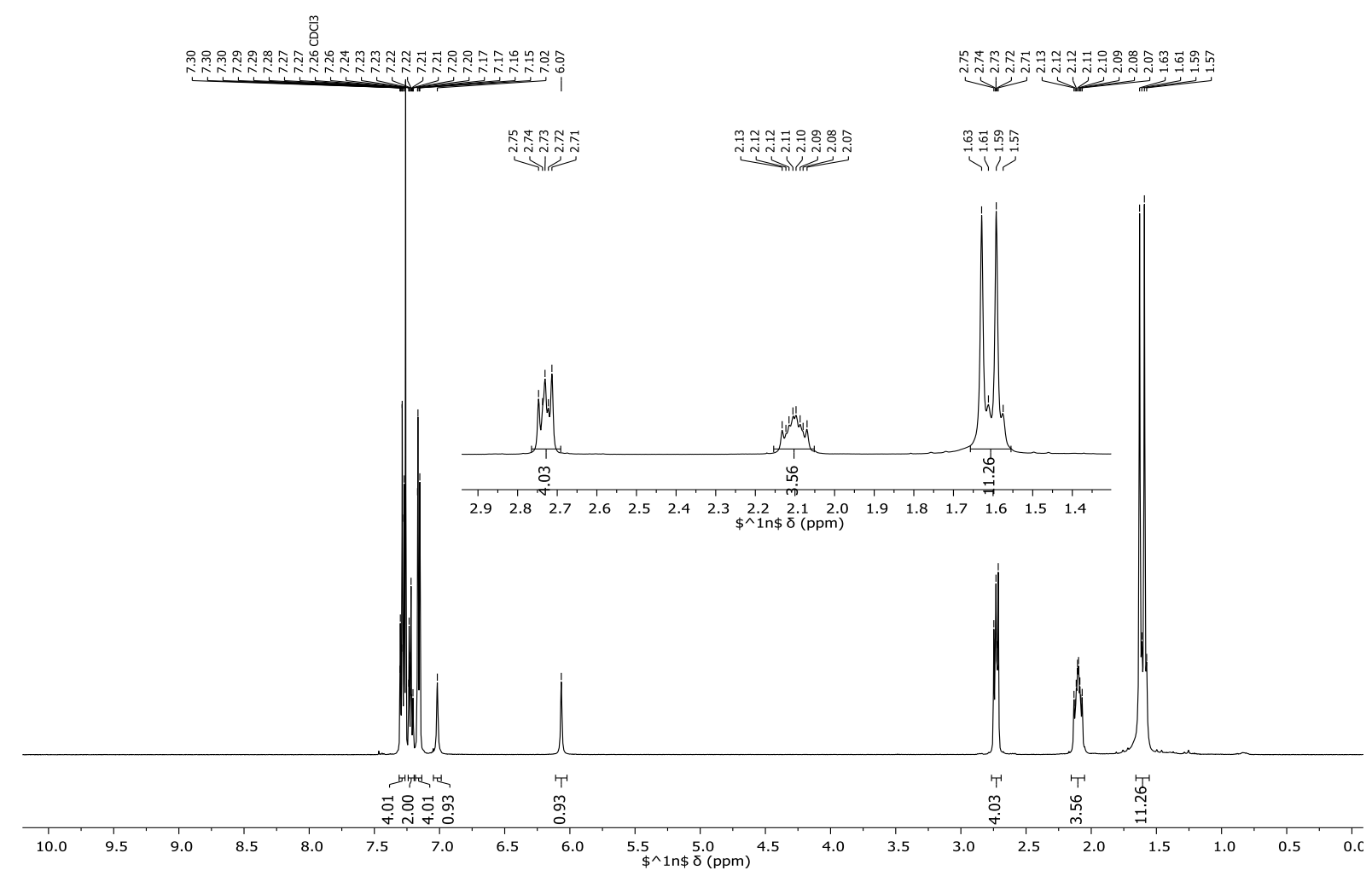

Figure S3. ${ }^{1} \mathrm{H}$ NMR spectrum of $\boldsymbol{d}_{\mathrm{n}}-\mathbf{1}$ f prepared from the reaction of $\boldsymbol{d}_{\mathbf{0}}-\mathbf{2}$ with $\mathrm{PD}_{\mathbf{3}}$. 


\section{Reaction of 2-methyl-4-phenylbut-2-yl acetate- $d_{6}\left(2-d_{6}\right)$ with $\mathrm{PH}_{3}$}

GP2 was performed using $d_{6}$-2-methyl-4-phenylbut-2-yl acetate $\boldsymbol{d}_{6}$-2 $(0.637 \mathrm{~g}, 3.00 \mathrm{mmol}) . \boldsymbol{d}_{\mathrm{n}}$-Di-(2methyl-4-phenylbut-2-yl)phosphonium trifluoromethanesulfonate $\boldsymbol{d}_{\mathrm{n}}-\mathbf{1 f}(\mathbf{0} .356 \mathrm{~g})$ was obtained as a colourless crystalline solid. The isotopic distribution of $\boldsymbol{d}_{\mathbf{n}}-\mathbf{1} \mathbf{f}$ prepared in this way was determined by ESI-MS (Figure S4) and ${ }^{1} \mathrm{H}$ NMR spectroscopy (Figure S5).

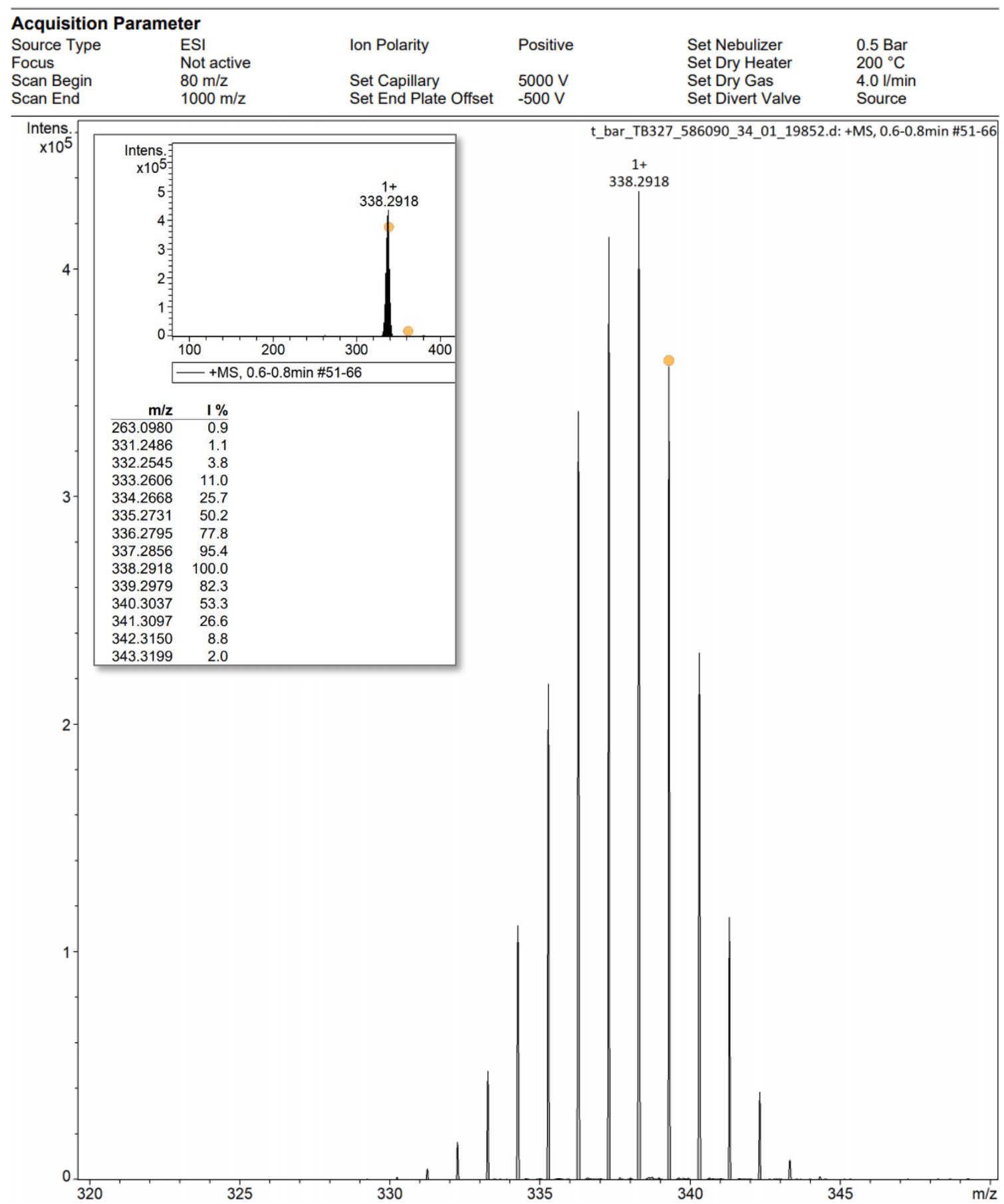

Figure S4. ESI-MS mass distribution for the $\boldsymbol{d}_{\mathrm{n}} \mathbf{- 1} \mathbf{f}$ cation prepared from the reaction of $\boldsymbol{d}_{\mathbf{6}} \mathbf{- 2}$ with $\mathrm{PH}_{3}$. 


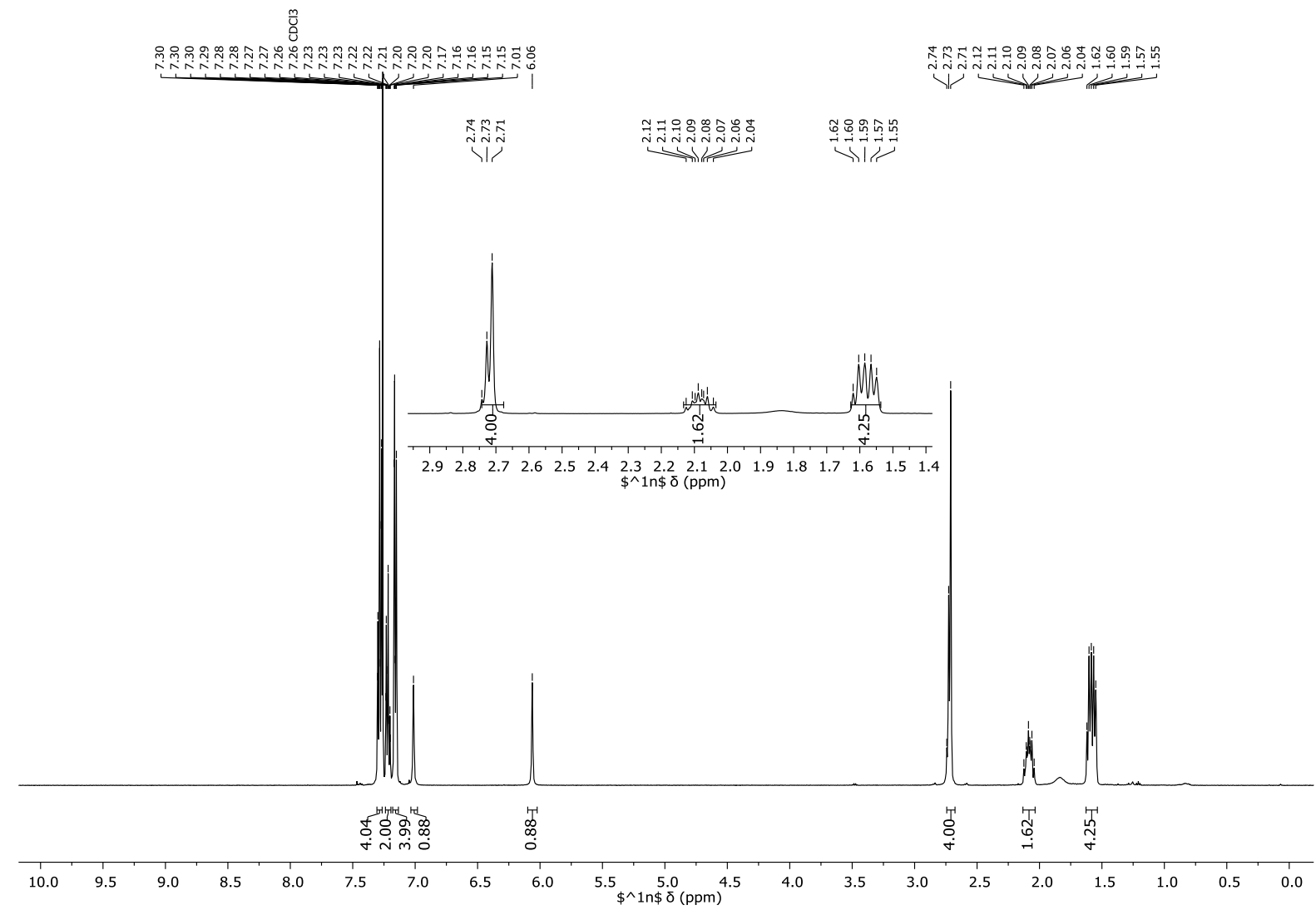

Figure S5. ${ }^{1} \mathrm{H}$ NMR spectrum of $\boldsymbol{d}_{\mathbf{n}}-\mathbf{1} \mathbf{f}$ prepared from the reaction of $\boldsymbol{d}_{\mathbf{6}}-\mathbf{2}$ with $\mathrm{PH}_{3}$. 


\section{Reaction of 2-methyl-4-phenylbut-2-yl acetate- $d_{6}\left(2-d_{6}\right)$ with $\mathrm{PD}_{3}$}

GP2 was followed, with $d_{6}$-2-methyl-4-phenylbut-2-yl acetate $2-d_{6}(0.637 \mathrm{~g}, 3.00 \mathrm{mmol})$ and DCl $(5.0$ $\mathrm{M}$ in $\left.\mathrm{D}_{2} \mathrm{O}\right) \cdot \boldsymbol{d}_{\mathrm{n}}$-Di-(2-methyl-4-phenylbut-2-yl)phosphonium trifluoromethanesulfonate $\boldsymbol{d}_{\mathrm{n}}-\mathbf{1 f}(0.208 \mathrm{~g})$ was obtained as a colourless crystalline solid. The isotopic distribution of $\boldsymbol{d}_{\mathbf{n}}-\mathbf{1} \mathbf{f}$ prepared in this way was determined by ESI-MS (Figure S6) and ${ }^{1} \mathrm{H}$ NMR spectroscopy (Figure S7).

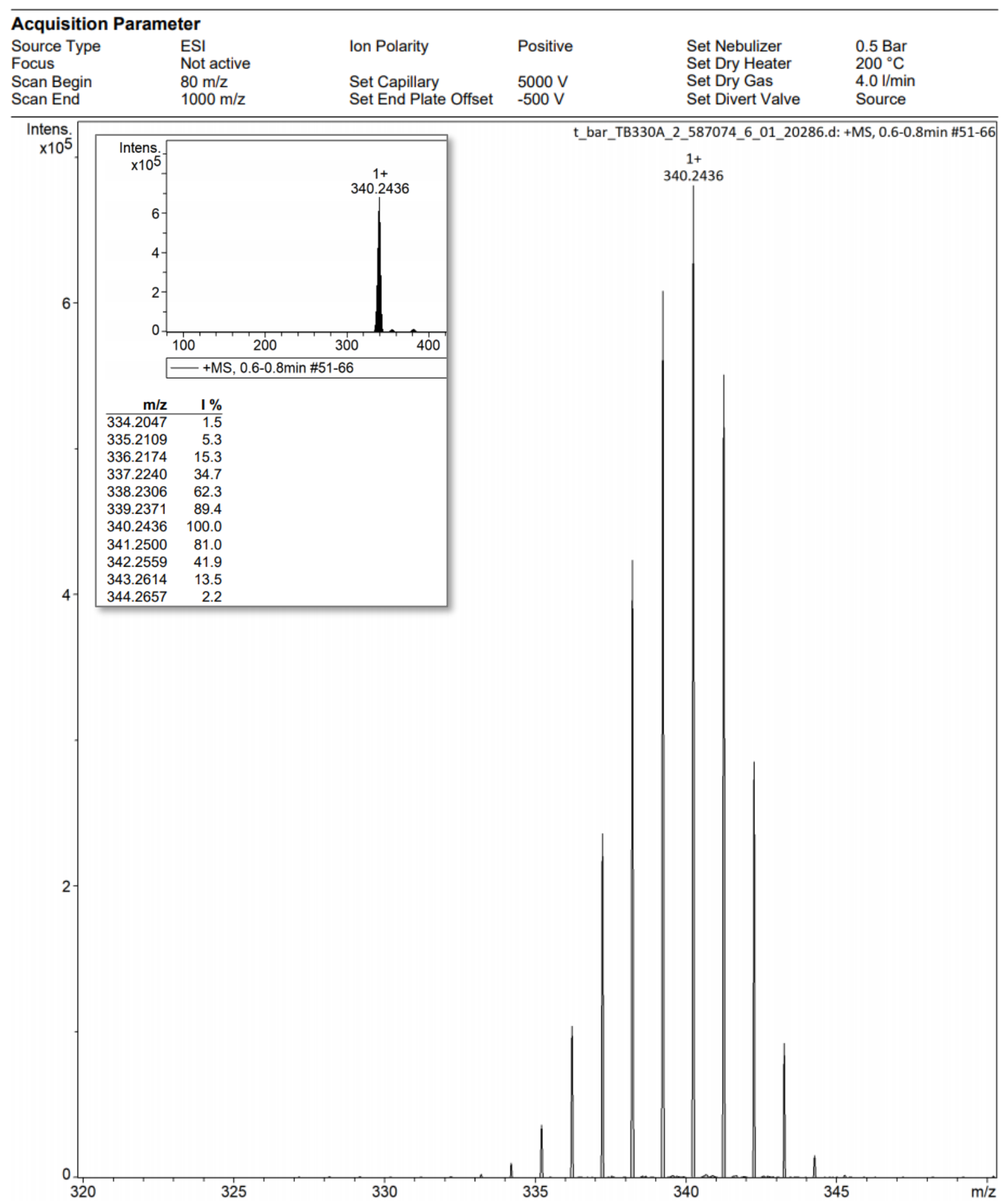

Figure S6. ESI-MS mass distribution for the $\boldsymbol{d}_{\mathbf{n}}-\mathbf{1} \mathbf{f}$ cation prepared from the reaction of $\boldsymbol{d}_{\mathbf{6}}-\mathbf{2}$ with $\mathrm{PD}_{3}$. 


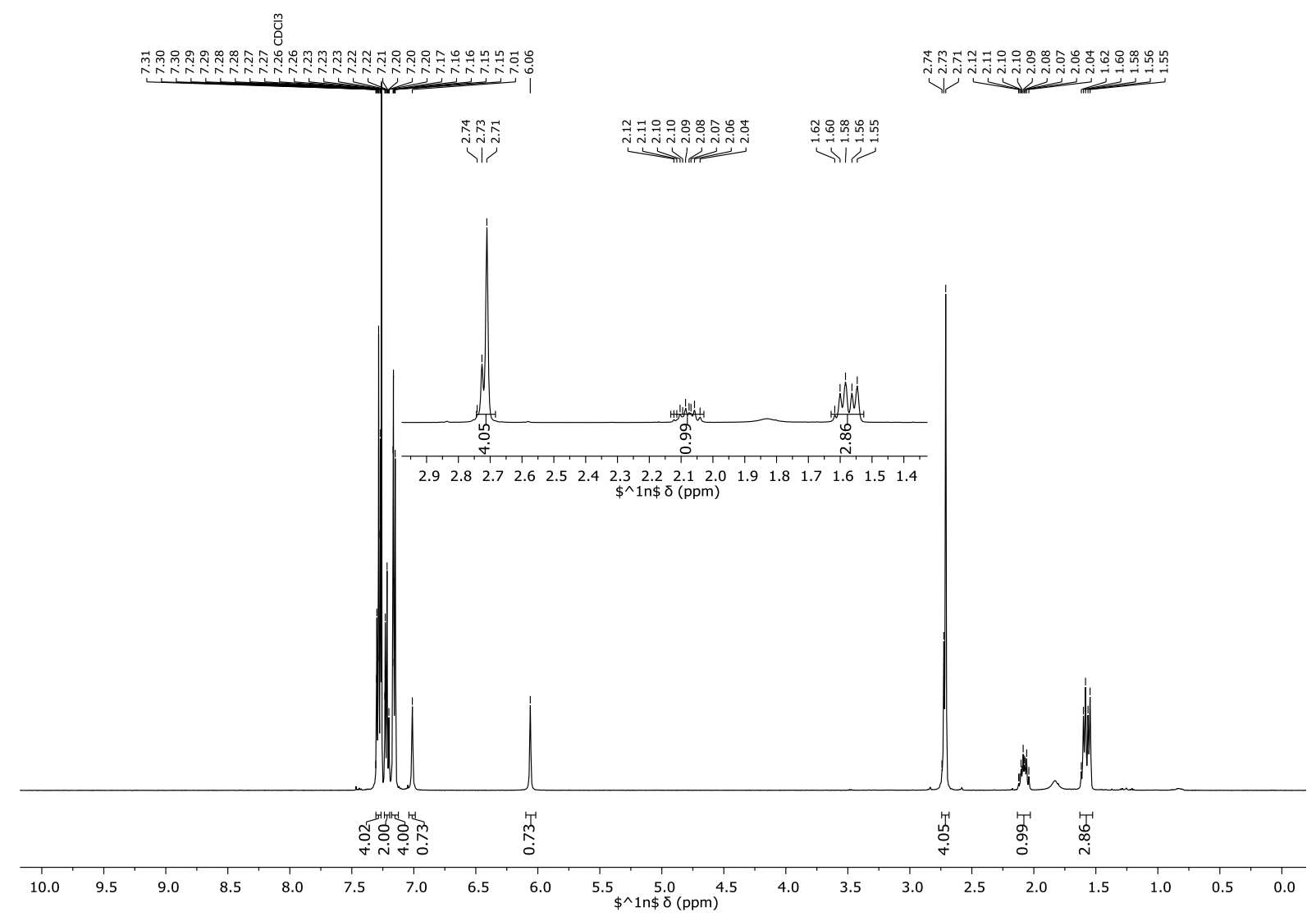

Figure S7. ${ }^{1} \mathrm{H}$ NMR spectrum of $\boldsymbol{d}_{\mathbf{n}}-\mathbf{1} \mathbf{f}$ prepared from the reaction of $\boldsymbol{d}_{\mathbf{6}}-\mathbf{2}$ with $\mathrm{PD}_{3}$. 


\section{Derivatisation of Di-(3-ethyl-3-pentyl)phosphonium Triflate $\underline{1 \mathrm{c}}$ (Manuscript Scheme 5)}

Di-(3-ethyl-3-pentyl)phosphine borane complex

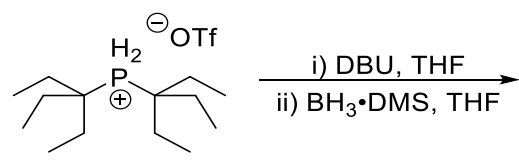<smiles>[B]P(C(CC)CC)C(CC)CC</smiles>

A flame-dried microwave tube containing di-(3-ethyl-3-pentyl)phosphonium trifluoromethanesulfonate $1 \mathrm{c}(0.190 \mathrm{~g}, 0.500 \mathrm{mmol})$ was evacuated and backfilled with anhydrous dinitrogen $(3 \times)$, then anhydrous degassed THF $(2.0 \mathrm{~mL})$ and DBU $(83.7 \mathrm{mg}, 0.550 \mathrm{mmol})$ were added. The mixture was stirred at r.t. for 10 minutes before borane dimethylsulfide complex (2.0 M IN THF; $0.50 \mathrm{~mL}, 1.00 \mathrm{mmol}$ ) was added and the mixture was stirred overnight at r.t. The vessel was opened to air, the solvent was removed in vacuo, and the resulting crude material was passed through a pad of silica gel (eluent: cyclohexane:Et ${ }_{2} \mathrm{O}, 5: 1$ ). After concentration in vacuo, di-(3-ethyl-3pentyl)phosphine borane complex $(0.118 \mathrm{~g}, 0.484 \mathrm{mmol}, 97 \%)$ was obtained as a low-melting colourless solid.

${ }^{1} \mathrm{H}$ NMR $\left(500 \mathrm{MHz}, \mathrm{CDCl}_{3}\right): \delta 4.41(\mathrm{dq}, J=353.6,6.9 \mathrm{~Hz}, 1 \mathrm{H}), 1.82-1.70(\mathrm{~m}, 12 \mathrm{H}), 0.95(\mathrm{t}, J=7.4 \mathrm{~Hz}$, $18 \mathrm{H}), 0.90-0.20$ (br. m, 3H).

${ }^{11}$ B NMR (160 MHz, $\left.\mathrm{CDCl}_{3}\right): \delta-42.5$ to $-38.5(\mathrm{~m})$.

${ }^{13} \mathrm{C}\left\{{ }^{1} \mathrm{H}\right\}$ NMR $\left(126 \mathrm{MHz}, \mathrm{CDCl}_{3}\right): \delta 42.9(\mathrm{~d}, J=20.2 \mathrm{~Hz}), 28.3(\mathrm{~d}, J=1.9 \mathrm{~Hz}), 8.8(\mathrm{~d}, J=5.2 \mathrm{~Hz})$.

${ }^{31}$ P NMR (202 MHz, $\left.\mathrm{CDCl}_{3}\right): \delta 12.66(\mathrm{dm}, J=356.5 \mathrm{~Hz})$.

$v_{\max }($ neat $) / \mathrm{cm}^{-1}: 2965,2939,2881,2398,2368,1451,1384,1330,1172,1143,1064,1039,970,927$, $884,858,801,743,695,608,556,504,427$.

HRMS: calcd. for $\mathrm{C}_{14} \mathrm{H}_{34} \mathrm{BP}+\mathrm{Na}^{+}: 267.2383[\mathrm{M}+\mathrm{Na}]^{+}$; found $\left(\mathrm{ESI}^{+}\right): 267.2381$. 


\section{Di-(3-ethyl-3-pentyl)chlorophosphine}<smiles>CCC(CC)(CC)[Pb]C(CC)(CC)CC</smiles>

A flame-dried microwave tube containing di-(3-ethyl-3-pentyl)phosphonium trifluoromethanesulfonate $1 \mathrm{c}(0.190 \mathrm{~g}, 0.500 \mathrm{mmol})$ was evacuated and backfilled with anhydrous dinitrogen $(3 \times)$, then anhydrous degassed $\mathrm{CCl}_{4}(2.0 \mathrm{~mL})$ and DBU $(76.1 \mathrm{mg}, 0.500 \mathrm{mmol})$ were added. The mixture was stirred for $27 \mathrm{~h}$ at $50{ }^{\circ} \mathrm{C}$. Stirring was then stopped to allow the biphasic mixture to separate; the lower layer was transferred into a flame-dried Schlenk tube (previously evacuated and backfilled with anhydrous dinitrogen $(3 \times))$ and the solvent was removed in vacuo giving di-(3-ethyl-3pentyl)chlorophosphine $(0.128 \mathrm{~g}, 0.481 \mathrm{mmol}, 96 \%)$ as a colourless liquid.

A 13-et is expected in the ${ }^{31} \mathrm{P}$ NMR spectrum, from ${ }^{3} \mathrm{~J}_{\mathrm{P}-\mathrm{H}}$ coupling. This is not observed in full as the lower intensity peaks are not observed due to poor signal:noise and as such is reported below as a multiplet with the coupling constant given.

${ }^{1} \mathrm{H}$ NMR $\left(500 \mathrm{MHz}, \mathrm{CDCl}_{3}\right): \delta 1.84-1.67(\mathrm{~m}, 12 \mathrm{H}), 0.98(\mathrm{t}, J=7.5 \mathrm{~Hz}, 18 \mathrm{H})$.

${ }^{13} \mathrm{C}\left\{{ }^{1} \mathrm{H}\right\}$ NMR (126 MHz, CDCl 3$): \delta 46.4(\mathrm{~d}, J=51.4 \mathrm{~Hz}), 27.89(\mathrm{~d}, J=15.4 \mathrm{~Hz}), 9.38(\mathrm{~d}, J=10.7 \mathrm{~Hz})$.

${ }^{31} \mathrm{P}$ NMR $\left(202 \mathrm{MHz}, \mathrm{CDCl}_{3}\right): \delta 158.34(\mathrm{~m}, J=8.9 \mathrm{~Hz})$. 


\section{Di-(3-ethyl-3-pentyl)phosphine oxide}

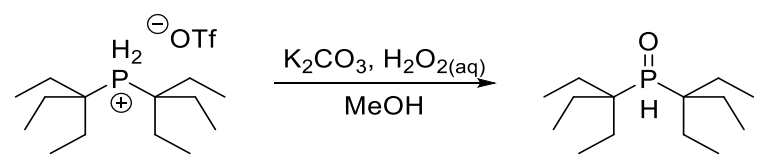

A suspension of di-(3-ethyl-3-pentyl)phosphonium trifluoromethanesulfonate 1c (76.1 mg, 0.200 $\mathrm{mmol})$ and $\mathrm{K}_{2} \mathrm{CO}_{3}(27.6 \mathrm{mg}, 0.200 \mathrm{mmol})$ in methanol $(1.0 \mathrm{~mL})$ was stirred for 30 minutes. $30 \%$ aqueous $\mathrm{H}_{2} \mathrm{O}_{2}(60 \mu \mathrm{L}, 0.50 \mathrm{mmol})$ was added and the mixture was stirred for $2 \mathrm{~h}$. A saturated aqueous solution of sodium metabisulfite $(0.5 \mathrm{~mL})$ and aqueous hydrochloric acid $(1 \mathrm{M} ; 1.0 \mathrm{~mL})$ were then added. The methanol was removed in vacuo and the remaining mixture was extracted with dichloromethane (3 x). The combined organic portions were concentrated in vacuo to give di-(3-ethyl-3-pentyl)phosphine oxide (46.9 mg, $0.190 \mathrm{mmol}, 95 \%$ ) as a colourless solid.

${ }^{1} \mathrm{H}$ NMR $\left(500 \mathrm{MHz}, \mathrm{CDCl}_{3}\right): \delta 6.60(\mathrm{~d}, J=421.4 \mathrm{~Hz}, 1 \mathrm{H}), 1.87-1.63(\mathrm{~m}, 12 \mathrm{H}), 1.00(\mathrm{t}, J=7.5 \mathrm{~Hz}, 18 \mathrm{H})$. ${ }^{13} \mathrm{C}\left\{{ }^{1} \mathrm{H}\right\}$ NMR $(126 \mathrm{MHz}, \mathrm{CDCl}): \delta 44.5(\mathrm{~d}, J=54.8 \mathrm{~Hz}), 26.0,8.5(\mathrm{~d}, J=6.1 \mathrm{~Hz})$.

${ }^{31}$ P NMR (202 MHz, $\left.\mathrm{CDCl}_{3}\right): \delta 53.88(\mathrm{dm}, J=421.5 \mathrm{~Hz})$.

$V_{\max }($ neat $) / \mathrm{cm}^{-1}: 2964,2940,2880,2294,1460,1441,1378,1333,1273,1176,1158,1134,1075,1033$, 997,983 ,915, 886, 803, 765, 735, 623, 552, 517, 485, 448, 415.

HRMS: calcd. for $\mathrm{C}_{14} \mathrm{H}_{31} \mathrm{OP}+\mathrm{H}^{+}: 247.2185[\mathrm{M}+\mathrm{H}]^{+}$; found $\left(\mathrm{ESI}^{+}\right)$: 247.2190 .

m.p. $/{ }^{\circ} \mathrm{C}: 34-35$. 


\section{Di-(3-ethyl-3-pentyl)benzylphosphine borane complex}

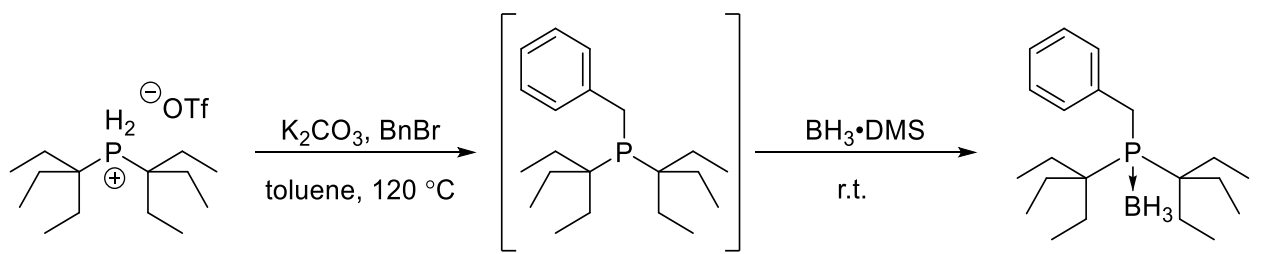

A flame-dried microwave tube containing di-(3-ethyl-3-pentyl)phosphonium trifluoromethanesulfonate $1 \mathrm{c}(0.190 \mathrm{~g}, 0.500 \mathrm{mmol})$ and $\mathrm{K}_{2} \mathrm{CO}_{3}(0.207 \mathrm{~g}, 1.50 \mathrm{mmol})$ was evacuated and backfilled with dry nitrogen $(3 \times)$ before anhydrous degassed toluene $(2.0 \mathrm{~mL})$ and benzyl bromide (85.5 mg, $0.500 \mathrm{mmol}$ ) were added. The mixture was stirred overnight at $120^{\circ} \mathrm{C}$, then allowed to cool without stirring so that the suspended solids settled. The supernatant was then transferred to a second flame-dried microwave tube containing borane dimethylsulfide complex (2.0 M in THF; 0.50 $\mathrm{mL}, 1.00 \mathrm{mmol})$. Anhydrous degassed toluene $(2.0 \mathrm{~mL})$ was added to the solid residue in the first tube, the suspension was stirred and allowed to settle, and the supernatant transferred to the microwave tube containing borane dimethylsulfide complex; this process was repeated a further two times. The mixture containing borane dimethylsulfide was stirred at r.t. for 2 h. The vessel was then opened to air, solvent was removed in vacuo, and the resulting crude material was passed through a pad of silica gel (eluent: cyclohexane: $\left.\mathrm{Et}_{2} \mathrm{O}, 5: 1\right)$. Removal of solvent in vacuo afforded di-(3-ethyl-3pentyl)benzylphosphine borane complex (0.143 g, $0.426 \mathrm{mmol}, 85 \%)$ as a colourless liquid.

${ }^{1} \mathrm{H}$ NMR (400 MHz, $\left.\mathrm{CDCl}_{3}\right): \delta$ 7.50-7.45 (m, 2H), 7.27-7.15 (m, 3H), $3.31(\mathrm{~d}, J=11.8 \mathrm{~Hz}, 2 \mathrm{H}), 1.93-1.82$ $(\mathrm{m}, 12 \mathrm{H}), 1.03-0.17(\mathrm{~m}, 21 \mathrm{H}$, including $\mathrm{t}, J=7.5 \mathrm{~Hz})$

${ }^{11}$ B NMR (128 MHz, $\left.\mathrm{CDCl}_{3}\right): \delta-39.5$ to $-34.5(\mathrm{~m})$.

${ }^{13} \mathrm{C}\left\{{ }^{1} \mathrm{H}\right\}$ NMR (101 MHz, $\left.\mathrm{CDCl}_{3}\right): \delta 135.7(\mathrm{~d}, J=3.6 \mathrm{~Hz}), 131.1(\mathrm{~d}, J=3.9 \mathrm{~Hz}), 127.9(\mathrm{~d}, J=1.8 \mathrm{~Hz}), 126.5$ $(\mathrm{d}, J=2.2 \mathrm{~Hz}), 44.3(\mathrm{~d}, J=17.1 \mathrm{~Hz}), 29.7(\mathrm{~d}, J=21.0 \mathrm{~Hz}), 27.4(\mathrm{~d}, J=1.7 \mathrm{~Hz}), 10.2(\mathrm{~d}, J=5.6 \mathrm{~Hz})$.

${ }^{31} \mathrm{P}\left\{{ }^{1} \mathrm{H}\right\}$ NMR (162 MHz, $\left.\mathrm{CDCl}_{3}\right): 53.31(\mathrm{~m})$.

$\mathbf{V}_{\max }\left(\right.$ neat)/cm ${ }^{-1}:$ 2969, 2940, 2881, 2399, 2302, 1601, 1496, 1454, 1382, 1339, 1156, 1064, 1032, 914 , $819,769,699,601,559,470,435$.

HRMS: calcd. for $\mathrm{C}_{21} \mathrm{H}_{40} \mathrm{BP}+\mathrm{Na}^{+}$: $357.2853[\mathrm{M}+\mathrm{Na}]^{+}$; found $\left(\mathrm{ESI}^{+}\right)$: 357.2852 . 


\section{Synthesis and Characterisation of Di-tert-alkylbiarylphosphines $\underline{\mathbf{3}}$ (Manuscript Scheme 6)}

\section{2-Biphenyl trifluoromethanesulfonate ${ }^{20}$}<smiles>CCOc1ccccc1-c1ccccc1OCCOCCO</smiles>

A flame-dried Schlenk tube containing 2-phenylphenol $(8.51 \mathrm{~g}, 50.0 \mathrm{mmol})$ was evacuated and backfilled with anhydrous dinitrogen $(3 \times)$, then anhydrous degassed dichloromethane $(50 \mathrm{~mL})$ and pyridine $(4.83 \mathrm{~mL}, 60.0 \mathrm{mmol})$ were added. The resulting solution was cooled to $0^{\circ} \mathrm{C}$ and trifluoromethanesulfonic anhydride $(10.1 \mathrm{~mL}, 50.0 \mathrm{mmol})$ was added slowly. The mixture was stirred at r.t. for $2 \mathrm{~h}$, then passed through a pad of silica gel (eluent: dichloromethane). The solvent was removed in vacuo and the resulting crude material was passed through another pad of silica gel (eluent: 19:1 petroleum ether : $\mathrm{Et}_{2} \mathrm{O}$ ). Concentration in vacuo afforded 2-biphenyl trifluoromethanesulfonate $(15.1 \mathrm{~g}, 50.0 \mathrm{mmol},>99 \%)$ as a colourless liquid. This material was used without further purification.

Characterisation data were consistent with literature values: ${ }^{1} \mathrm{H},{ }^{20}{ }^{13} \mathrm{C}\left\{{ }^{1} \mathrm{H}\right\},{ }^{20}$ and ${ }^{19} \mathrm{~F}^{20} \mathrm{NMR}$.

${ }^{1} \mathrm{H}$ NMR (500 MHz, $\left.\mathrm{CDCl}_{3}\right):$ 7.51-7.39 (m, 9H).

${ }^{13} \mathrm{C}\left\{{ }^{1} \mathrm{H}\right\}$ NMR $\left(126 \mathrm{MHz}, \mathrm{CDCl}_{3}\right): \delta 147.0,135.7,132.1,129.5,129.1,128.7,128.6,128.5,122.2,118.5$ (q, $J=320.6 \mathrm{~Hz}$ ).

${ }^{19} \mathrm{~F} \mathrm{NMR}\left(470 \mathrm{MHz}, \mathrm{CDCl}_{3}\right): \delta-74.08$.

$v_{\max }($ neat $) / \mathrm{cm}^{-1}: 3066,1477,1419,1246,1203,1135,1099,1046,1011,881,783,764,751,731,698$, $625,593,570,503,462$.

HRMS: calcd. for $\mathrm{C}_{13} \mathrm{H}_{9} \mathrm{~F}_{3} \mathrm{O}_{3} \mathrm{~S}+\mathrm{Na}^{+}: 325.0117[\mathrm{M}+\mathrm{Na}]^{+}$; found $\left(\mathrm{ESI}^{+}\right)$: 325.0118 . 


\section{General procedure for P-C cross couplings with 2-biphenyl triflate (GP3)}

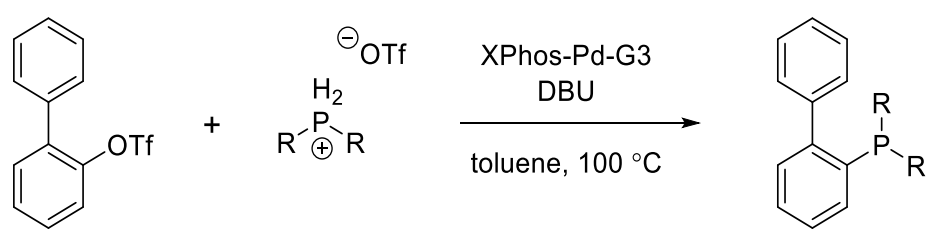

A microwave tube containing XPhosPdG3 ${ }^{21}(8.5 \mathrm{mg}, 10 \mu \mathrm{mol}, 2 \mathrm{~mol} \%)$ and di-tert-alkylphosphonium trifluoromethanesulfonate $1(0.500 \mathrm{mmol})$ was evacuated and backfilled with anhydrous dinitrogen $(3 \times)$, then degassed toluene $(2.50 \mathrm{~mL}), 2$-biphenyl trifluoromethanesulfonate $(151 \mathrm{mg}, 0.500 \mathrm{mmol})$, and DBU $(0.224 \mathrm{~mL}, 1.50 \mathrm{mmol})$ were added. The mixture was stirred at $100{ }^{\circ} \mathrm{C}$ for $16 \mathrm{~h}$, then allowed to cool to r.t. The reaction was diluted with $\mathrm{Et}_{2} \mathrm{O}$ and washed with water. The aqueous portion was extracted with $\mathrm{Et}_{2} \mathrm{O}(2 \times)$, and the combined organic portions were dried $\left(\mathrm{MgSO}_{4}\right)$, filtered and concentrated in vacuo. Recrystallisation from methanol afforded the pure dialkylbiarylphosphine 3.

\section{General procedure for P-C cross couplings with 2-biphenyl bromide (GP4)}<smiles>Brc1ccccc1-c1ccccc1</smiles>

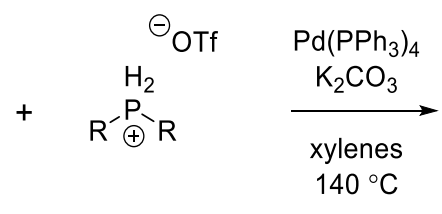<smiles>[R]P(c1ccccc1)c1ccccc1</smiles>

A microwave tube containing $\mathrm{Pd}_{\left(\mathrm{PPh}_{3}\right)_{4}} \quad(3$ mol\%), di-tert-alkylphosphonium trifluoromethanesulfonate 1 (1.00 equiv.) and $\mathrm{K}_{2} \mathrm{CO}_{3}$ (3.00 equiv.) was evacuated and backfilled with anhydrous dinitrogen $(3 \times)$, then degassed xylenes (to give $[1]_{0}=0.4-0.5 \mathrm{M}$ ) and 2-biphenyl bromide (1.20 equiv.) were added. The mixture was stirred at $140{ }^{\circ} \mathrm{C}$ for $16 \mathrm{~h}$, then allowed to cool to r.t. The reaction was diluted with $\mathrm{Et}_{2} \mathrm{O}$ and washed with water. The aqueous portion was extracted with $\mathrm{Et}_{2} \mathrm{O}$ $(2 \times)$, and the combined organic portions were dried $\left(\mathrm{MgSO}_{4}\right)$, filtered and concentrated in vacuo. Recrystallisation from methanol or acetone afforded the pure dialkylbiarylphosphine 3.

Note: di-tert-alkylbiarylphosphines $\mathbf{3}$ obtained by GP3 or GP4 that could not be isolated by recrystallisation were employed directly in the synthesis of the corresponding di-tert- 
alkylbiarylphosphine selenides 4 and di-tert-alkylbiarylphosphine gold(I) chloride complexes 5 . Details of these procedures are provided in Sections S10 and S11, below. 


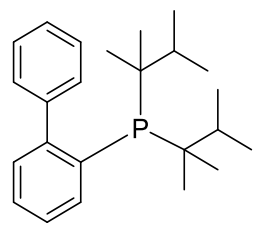

GP3 was performed using bis-(2,3-dimethyl-2-butyl)phosphonium trifluoromethanesulfonate 1d (0.138 g, 0.500 mmol). 2-(Bis-(2,3-dimethyl-2-butyl)phosphino)biphenyl 3d (0.098 g, 0.276 mmol, $55 \%)$ was isolated as an off-white crystalline solid.

${ }^{1} \mathrm{H}$ NMR (500 MHz, $\left.\mathrm{CDCl}_{3}\right): \delta 7.94(\mathrm{dt}, J=7.7,1.6 \mathrm{~Hz}, 1 \mathrm{H}), 7.39-7.35(\mathrm{~m}, 1 \mathrm{H}), 7.34-7.26(\mathrm{~m}, 7 \mathrm{H}), 1.66$ (app. dq, $J=13.4,6.9 \mathrm{~Hz}, 2 \mathrm{H}$ ), $1.25(\mathrm{~d}, J=8.1 \mathrm{~Hz}, 6 \mathrm{H}), 0.92(\mathrm{~d}, J=8.2 \mathrm{~Hz}, 6 \mathrm{H}), 0.84(\mathrm{~d}, J=6.8 \mathrm{~Hz}, 6 \mathrm{H}$ ), $0.73(d, J=6.7 \mathrm{~Hz}, 6 \mathrm{H})$.

${ }^{13} \mathrm{C}\left\{{ }^{1} \mathrm{H}\right\}$ NMR $(126 \mathrm{MHz}, \mathrm{CDCl}): \delta 151.9(\mathrm{~d}, J=34.5 \mathrm{~Hz}), 144.0(\mathrm{~d}, J=7.2 \mathrm{~Hz}), 136.6(\mathrm{~d}, J=1.7 \mathrm{~Hz}), 135.7$ (d, $J=32.3 \mathrm{~Hz}$ ), 131.0 (d, J = 4.1 Hz), 130.7 (d, J = 6.5 Hz), 128.4, 127.0, 126.2, 125.7, 39.8 (d, J = 31.0 $\mathrm{Hz}$ ), $35.8(\mathrm{~d}, J=22.2 \mathrm{~Hz}), 23.6(\mathrm{~d}, J=9.5 \mathrm{~Hz}), 23.2(\mathrm{~d}, J=6.8 \mathrm{~Hz}), 18.1(\mathrm{~d}, J=12.8 \mathrm{~Hz}), 17.8(\mathrm{~d}, J=14.8$ $\mathrm{Hz})$.

${ }^{31} \mathrm{P}\left\{{ }^{1} \mathrm{H}\right\}$ NMR (202 MHz, $\left.\mathrm{CDCl}_{3}\right): \delta 3.14(\mathrm{~s})$.

$v_{\max }($ neat $) / \mathrm{cm}^{-1}: 3051,2974,2958,2898,2867,1463,1442,1375,1160,1122,1079,1050,1008,995$, $909,870,778,746,695,616,555,514,476$.

HRMS: calcd. for $\mathrm{C}_{24} \mathrm{H}_{35} \mathrm{P}+\mathrm{H}^{+}$: $355.2549[\mathrm{M}+\mathrm{H}]^{+}$; found $(\mathrm{ESI})^{+}$): 355.2544 .

m.p./ ${ }^{\circ} \mathrm{C}: 64-66$. 


\section{2-(Di-(2-methyl-4-phenyl-2-butyl)phosphino)biphenyl (3f)}<smiles>CC(C)(CCc1ccccc1)P(c1ccccc1)C(C)(C)CCc1ccccc1</smiles>

GP3 was performed using di-(2-methyl-4-phenyl-2-butyl)phosphonium trifluoromethanesulfonate 1 ff (0.238 g, 0.500 mmol). 2-(Di-(2-methyl4-phenyl-2-butyl)phosphino)biphenyl $3 f(0.162 \mathrm{~g}, 0.338 \mathrm{mmol}$, $68 \%)$ was isolated as an off-white crystalline solid.

${ }^{1} \mathrm{H}$ NMR $\left(500 \mathrm{MHz}, \mathrm{CDCl}_{3}\right): \delta 7.94(\mathrm{dt}, J=7.7,1.6 \mathrm{~Hz}, 1 \mathrm{H}), 7.40(\mathrm{t}, J=7.4 \mathrm{~Hz}, 1 \mathrm{H}), 7.34(\mathrm{td}, J=7.5,1.6$ $\mathrm{Hz}, 1 \mathrm{H}), 7.28-7.21(\mathrm{~m}, 10 \mathrm{H}), 7.15(\mathrm{t}, J=7.4 \mathrm{~Hz}, 2 \mathrm{H}), 7.06-7.02(\mathrm{~m}, 4 \mathrm{H}), 2.69-2.62(\mathrm{~m}, 2 \mathrm{H}), 2.57-2.49(\mathrm{~m}$, $2 \mathrm{H}), 1.80-1.70(\mathrm{~m}, 4 \mathrm{H}), 1.34(\mathrm{~d}, J=10.1 \mathrm{~Hz}, 6 \mathrm{H}), 1.16(\mathrm{~d}, J=10.3 \mathrm{~Hz}, 6 \mathrm{H})$.

${ }^{13} \mathrm{C}\left\{{ }^{1} \mathrm{H}\right\}$ NMR (126 MHz, CDCl 3 ): $\delta 151.6(\mathrm{~d}, J=33.2 \mathrm{~Hz}), 143.7(\mathrm{~d}, J=7.1 \mathrm{~Hz}), 143.3,136.9$ (d, $J=2.5$ $\mathrm{Hz}$ ), $134.7(\mathrm{~d}, J=29.2 \mathrm{~Hz}$ ), $131.0(\mathrm{~d}, J=6.4 \mathrm{~Hz}), 130.8(\mathrm{~d}, J=4.1 \mathrm{~Hz}), 128.7,128.5,128.4,127.2,126.5$, 126.0, 125.6, $45.2(\mathrm{~d}, J=20.0 \mathrm{~Hz}$ ), $36.6(\mathrm{~d}, J=28.6 \mathrm{~Hz}), 30.3(\mathrm{~d}, J=13.9 \mathrm{~Hz}), 27.5(\mathrm{~d}, J=11.1 \mathrm{~Hz}), 27.4$ $(\mathrm{d}, J=12.3 \mathrm{~Hz})$.

${ }^{31} \mathrm{P}\left\{{ }^{1} \mathrm{H}\right\}$ NMR (202 MHz, $\left.\mathrm{CDCl}_{3}\right): \delta 13.81(\mathrm{~s})$.

$V_{\max }\left(\right.$ neat) $/ \mathrm{cm}^{-1}:$ 3082, 3057, 3020, 2959, 2939, 2860, 1600, 1582, 1495, 1453, 380, 1362, 1176, 1129, $1069,1030,1008,908,840,745,696,600,577,508,492,460$.

HRMS: calcd. for $\mathrm{C}_{34} \mathrm{H}_{39} \mathrm{P}+\mathrm{H}^{+}$: $479.2862[\mathrm{M}+\mathrm{H}]^{+}$; found $\left.(\mathrm{ESI})^{+}\right): 479.2874$.

m.p./ ${ }^{\circ} \mathrm{C}: 85-86$. 


\section{2-(Di-(1-methylcyclopentyl)phosphino)biphenyl (3h)}

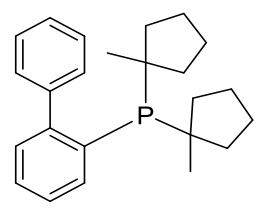

GP3 was performed using di-(1-methylcyclopentyl)phosphonium trifluoromethanesulfonate $1 \mathrm{~h}$ (0.174 g, $0.500 \mathrm{mmol}$ ). 2-(Di-(1-methylcyclopentyl)phosphino)biphenyl $3 \mathrm{~h}(0.115 \mathrm{~g}, 0.328 \mathrm{mmol}, 66 \%)$ was isolated as an off-white crystalline solid.

${ }^{1}{ }^{H}$ NMR $\left(500 ~ M H z, \mathrm{CDCl}_{3}\right): \delta 7.94(\mathrm{dt}, J=7.6,1.7 \mathrm{~Hz}, 1 \mathrm{H}), 7.27-7.38(\mathrm{~m}, 7 \mathrm{H}), 7.23-7.26$ (ddd, $J=7.4$, 4.0, $1.7 \mathrm{~Hz}, 1 \mathrm{H}), 1.76-1.86(\mathrm{~m}, 2 \mathrm{H}), 1.42-1.69(\mathrm{~m}, 12 \mathrm{H}), 1.31-1.37(\mathrm{~m}, 2 \mathrm{H}), 1.22(\mathrm{~d}, J=8.3 \mathrm{~Hz}, 6 \mathrm{H})$.

${ }^{13} \mathrm{C}\left\{{ }^{1} \mathrm{H}\right\}$ NMR $\left(126 \mathrm{MHz}, \mathrm{CDCl}_{3}\right): \delta 150.9(\mathrm{~d}, J=31.3 \mathrm{~Hz}), 143.9(\mathrm{~d}, J=5.9 \mathrm{~Hz}), 136.4(\mathrm{~d}, J=28.1 \mathrm{~Hz})$, $135.7(d, J=3.2 \mathrm{~Hz}), 130.9(\mathrm{~d}, J=3.2 \mathrm{~Hz}), 130.8(\mathrm{~d}, J=5.9 \mathrm{~Hz}), 128.3,127.3,126.5,125.9,42.3$ (d, $J=$ $23.7 \mathrm{~Hz}), 40.6(\mathrm{~d}, J=24.4 \mathrm{~Hz}), 40.0(\mathrm{~d}, J=17.1 \mathrm{~Hz}), 25.9(\mathrm{~d}, J=7.3 \mathrm{~Hz}), 24.4(\mathrm{~d}, J=8.2 \mathrm{~Hz}), 23.7(\mathrm{~d}, J=$ $7.5 \mathrm{~Hz})$.

${ }^{31} \mathrm{P}\left\{{ }^{1} \mathrm{H}\right\}$ NMR (202 MHz, $\left.\mathrm{CDCl}_{3}\right): \delta 18.31(\mathrm{~s})$.

$v_{\max }($ neat $) / \mathrm{cm}^{-1}: 3050,2954,2864,1443,1376,1315,1241,1216,1073,1007,910,829,775,743,701$, $617,505,473$.

HRMS: calcd. for $\mathrm{C}_{24} \mathrm{H}_{31} \mathrm{P}+\mathrm{H}^{+}: 351.2236[\mathrm{M}+\mathrm{H}]^{+}$; found $\left(\mathrm{ESI}^{+}\right): 351.2242$.

m.p./ ${ }^{\circ} \mathrm{C}: 79-81$. 


\section{2-(Di-(1-methylcyclohexyl)phosphino)biphenyl (3i)}

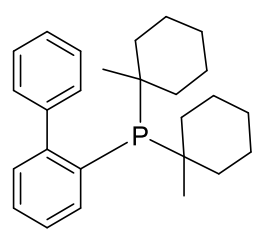

GP3 was performed using di-(1-methylcyclohexyl)phosphonium trifluoromethanesulfonate $\mathbf{1 i}$ (0.188 g, $0.500 \mathrm{mmol})$. 2-(Di-(1-methylcyclohexyl)phosphino)biphenyl 3i (0.114 g, $0.301 \mathrm{mmol}, 60 \%)$ was isolated as an off-white crystalline solid. The product could not be separated from $2 \%$ residual XPhos $\left(\delta_{\mathrm{p}}-12.31 \mathrm{ppm}\right)$.

${ }^{1} \mathrm{H}$ NMR $\left(500 \mathrm{MHz}, \mathrm{CDCl}_{3}\right): \delta 7.92(\mathrm{dt}, J=7.6,1.6 \mathrm{~Hz}, 1 \mathrm{H}), 7.38-7.23(\mathrm{~m}, 8 \mathrm{H}), 1.80-1.72(\mathrm{~m}, 2 \mathrm{H}), 1.66-$ $1.60(\mathrm{~m}, 2 \mathrm{H}), 1.52-1.41(\mathrm{~m}, 8 \mathrm{H}), 1.36-1.29(\mathrm{~m}, 6 \mathrm{H}), 1.26(\mathrm{~d}, J=8.8 \mathrm{~Hz}, 6 \mathrm{H}), 1.18-1.10(\mathrm{~m}, 2 \mathrm{H})$.

${ }^{13} \mathrm{C}\left\{{ }^{1} \mathrm{H}\right\}$ NMR $\left(126 \mathrm{MHz}, \mathrm{CDCl}_{3}\right): \delta 151.8(\mathrm{~d}, J=32.6 \mathrm{~Hz}), 144.1(\mathrm{~d}, J=6.7 \mathrm{~Hz}), 136.8(\mathrm{~d}, J=2.4 \mathrm{~Hz}), 134.5$ (d, $J=31.0 \mathrm{~Hz}$ ), 130.9 (d, J = 3.9 Hz), 130.8 (d, J = 6.1 Hz), 128.3, 127.2, 126.4, 125.5, 37.8 (d, J = 18.3 $\mathrm{Hz}), 37.6,37.5,37.4,26.3,22.7(\mathrm{~d}, J=7.9 \mathrm{~Hz}), 21.6(\mathrm{~d}, J=10.7 \mathrm{~Hz})$.

${ }^{31} \mathrm{P}\left\{{ }^{1} \mathrm{H}\right\}$ NMR (202 MHz, $\left.\mathrm{CDCl}_{3}\right): \delta 25.16$ (br. s.).

$v_{\max }($ neat $) / \mathrm{cm}^{-1}:$ 3054, 2916, 2847, 2665, 1442, 1377, 1339, 1284, 1162 ,1123, 1073, 963, 926, 850, $772,744,698,615,523,490,461$.

HRMS: calcd. for $\mathrm{C}_{26} \mathrm{H}_{35} \mathrm{P}+\mathrm{H}^{+}$: $379.2549[\mathrm{M}+\mathrm{H}]^{+}$; found $\left.(\mathrm{ESI})^{+}\right)$: 379.2549.

m.p./ ${ }^{\circ} \mathrm{C}: 77-79$. 


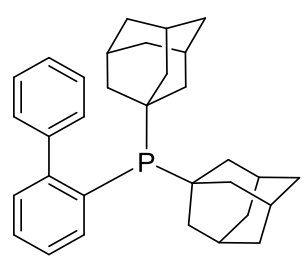

GP3 was performed using di-(1-adamantyl)phosphonium trifluoromethanesulfonate $1 \mathbf{k}(0.226 \mathrm{~g}$, $0.500 \mathrm{mmol}$ ). 2-(Di-(1-adamantyl)phosphino)biphenyl 3k (0.161 g, $0.354 \mathrm{mmol}, 71 \%)$ was isolated as an off-white crystalline solid.

Characterisation data were consistent with literature values: ${ }^{1} \mathrm{H},{ }^{22}{ }^{13} \mathrm{C}\left\{{ }^{1} \mathrm{H}\right\},{ }^{22}{ }^{31} \mathrm{P}\left\{{ }^{1} \mathrm{H}\right\}^{22} \mathrm{NMR}, \mathrm{IR} .{ }^{22}$

${ }^{1} \mathrm{H}$ NMR $\left(500 \mathrm{MHz}, \mathrm{CDCl}_{3}\right): \delta 7.90(\mathrm{dt}, J=7.3,1.2 \mathrm{~Hz}, 1 \mathrm{H}), 7.40-7.29(\mathrm{~m}, 5 \mathrm{H}), 7.28-7.22(\mathrm{~m}, 3 \mathrm{H}), 1.93-$ $1.82(\mathrm{~m}, 18 \mathrm{H}), 1.68-1.63(\mathrm{~m}, 12 \mathrm{H})$.

${ }^{13} \mathrm{C}\left\{{ }^{1} \mathrm{H}\right\}$ NMR $\left(126 \mathrm{MHz}, \mathrm{CDCl}_{3}\right): \delta 152.0(\mathrm{~d}, J=32.7 \mathrm{~Hz}), 144.1(\mathrm{~d}, J=7.2 \mathrm{~Hz}), 136.8(\mathrm{~d}, J=2.8 \mathrm{~Hz}), 133.2$ $(d, J=27.7 \mathrm{~Hz}), 130.8(\mathrm{~d}, J=6.2 \mathrm{~Hz}), 130.8(\mathrm{~d}, J=3.8 \mathrm{~Hz}), 128.3,127.2,126.4,125.5,42.0$ (d, J = 13.0 $\mathrm{Hz}), 37.5(\mathrm{~d}, J=25.6 \mathrm{~Hz}), 37.1,29.0(\mathrm{~d}, J=8.5 \mathrm{~Hz})$.

${ }^{31} \mathrm{P}\left\{{ }^{1} \mathrm{H}\right\} \mathrm{NMR}\left(202 \mathrm{MHz}, \mathrm{CDCl}_{3}\right): \delta 21.10(\mathrm{~s})$.

$V_{\max }\left(\right.$ neat) $/ \mathrm{cm}^{-1}:$ 2897, 2844, 2675, 1444, 1342, 1301, 1252, 1101, 1071, 1047, 1008, 970, 825, 770, $744,696,552,504,471,426$.

HRMS: calcd. for $\mathrm{C}_{34} \mathrm{H}_{39} \mathrm{P}+\mathrm{H}^{+}: 455.2862[\mathrm{M}+\mathrm{H}]^{+}$; found $\left(\mathrm{ESI}^{+}\right): 455.2865$.

m.p. $/{ }^{\circ} \mathrm{C}: 218-223$, lit. $^{22} 222-224$. 


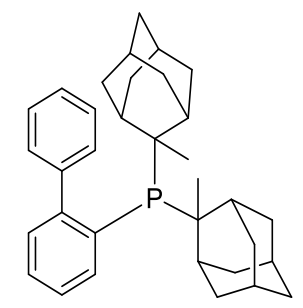

GP4 was performed using $\mathrm{Pd}\left(\mathrm{PPh}_{3}\right)_{4}(6.9 \mathrm{mg}, 6.0 \mu \mathrm{mol})$, di-(2-methyl-2-adamantyl)phosphonium trifluoromethanesulfonate 1 l (96.1 mg, $0.200 \mathrm{mmol})$, 2-bromobiphenyl (45 $\mu \mathrm{L}, 0.240 \mathrm{mmol}), \mathrm{K}_{2} \mathrm{CO}_{3}$ (82.9 $\mathrm{mg}, \quad 0.600 \mathrm{mmol})$ and degassed xylenes $(0.5 \mathrm{~mL})$. 2-(Di-(2-methyladamant-2yl)phosphino)biphenyl 31 (56.3 mg, $0.117 \mathrm{mmol}, 58 \%)$ was obtained as an off-white solid after recrystallisation from methanol.

${ }^{1} \mathrm{H}$ NMR $\left(500 \mathrm{MHz}, \mathrm{CDCl}_{3}\right): \delta 7.90(\mathrm{~d}, J=7.7 \mathrm{~Hz}, 1 \mathrm{H}), 7.44-7.18(\mathrm{~m}, 8 \mathrm{H}), 2.83(\mathrm{~d}, J=12.4 \mathrm{~Hz}, 2 \mathrm{H}), 2.26-$ $2.06(\mathrm{~m}, 6 \mathrm{H}), 1.85-1.71(\mathrm{~m}, 6 \mathrm{H}), 1.66-1.50(\mathrm{~m}, 12 \mathrm{H}), 1.49-1.41(\mathrm{~m}, 4 \mathrm{H}), 1.30(\mathrm{dt}, J=13.1,2.8 \mathrm{~Hz}, 2 \mathrm{H})$, $1.00(\mathrm{dt}, J=12.6,2.9 \mathrm{~Hz}, 2 \mathrm{H})$.

${ }^{13} \mathrm{C}\left\{{ }^{1} \mathrm{H}\right\}$ NMR (126 MHz, CDCl 3$): \delta 151.1(\mathrm{~d}, J=34.2 \mathrm{~Hz}), 144.1(\mathrm{~d}, J=5.3 \mathrm{~Hz}), 138.0,134.2(\mathrm{~d}, J=38.3$ $\mathrm{Hz}$ ), 131.6 (d, J = 6.7 Hz), 131.5 (d, $J=3.2 \mathrm{~Hz}$ ), 128.2, 127.5, 126.3, 125.3, 45.1 (d, J = 36.3 Hz), 40.1 (d, $J=3.2 \mathrm{~Hz}), 36.4(\mathrm{~d}, J=16.0 \mathrm{~Hz}), 35.9(\mathrm{~d}, J=9.5 \mathrm{~Hz}), 34.5(\mathrm{~d}, J=17.9 \mathrm{~Hz}), 33.6(\mathrm{~d}, J=15.0 \mathrm{~Hz}), 32.8$ (d, J $=5.4 \mathrm{~Hz}), 32.5(\mathrm{~d}, J=4.0 \mathrm{~Hz}), 28.3(\mathrm{~d}, J=1.4 \mathrm{~Hz}), 27.6,22.5$.

${ }^{31} \mathrm{P}\left\{{ }^{1} \mathrm{H}\right\}$ NMR $\left(202 \mathrm{MHz}, \mathrm{CDCl}_{3}\right): \delta-0.09(\mathrm{~s})$.

$v_{\max }($ neat $) / \mathrm{cm}^{-1}:$ 2896, 2844, 2663, 1441, 1380, 1351, 1304, 1221, 1098, 1072, 1021, 956, 868, 774, $744,696,615,552,486,465,450$.

HRMS: calcd. for $\mathrm{C}_{34} \mathrm{H}_{43} \mathrm{P}+\mathrm{H}^{+}: 483.3175[\mathrm{M}+\mathrm{H}]^{+}$; found $\left(\mathrm{ESI}^{+}\right)$: 483.3180 .

m.p./ ${ }^{\circ} \mathrm{C}: 169-171$. 


\section{tert-Alkyl Group Contributions to ${ }^{31} \mathrm{P}$ NMR Chemical Shifts}

Group contributions of individual tert-alkyl substituents to ${ }^{31} \mathrm{P}$ NMR chemical shifts were calculated according to the method of Grim et al. ${ }^{23}$ The excellent correlation observed between the calculated group contributions and the observed chemical shifts of JohnPhos analogues $\mathbf{3}$ does not hold for phosphines featuring two substituents at the $\mathrm{C}_{\beta}$-position (Figure $\mathrm{S} 8$ ).

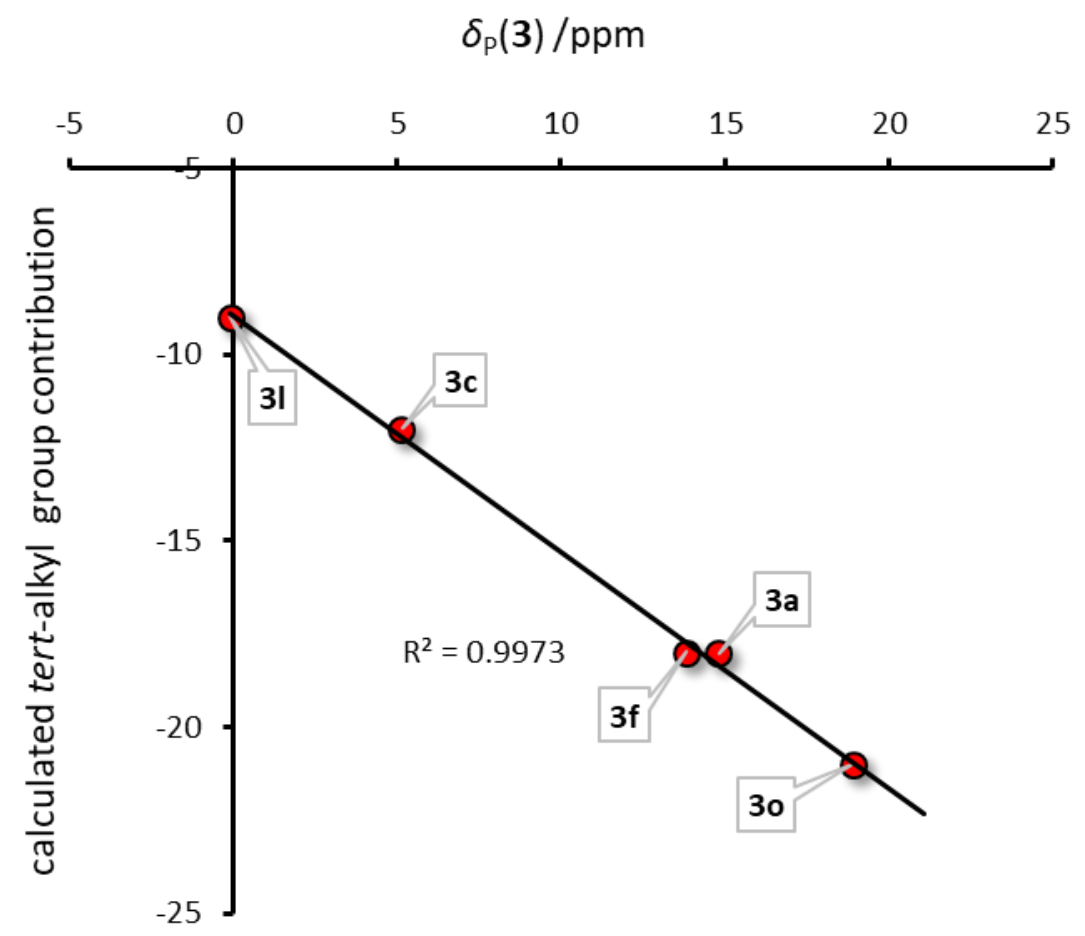

Figure S8. Correlation of calculated tert-alkyl group contributions vs observed ${ }^{31} \mathrm{P} N M R$ chemical shifts (/ppm) of JohnPhos analogues 3. 


\section{Synthesis of Di-tert-alkylbiarylphosphine Selenides $\underline{4}$ (Manuscript Scheme 6)}

General procedure for the synthesis of di-tert-alkylbiarylphosphine selenides (4) (GP5)

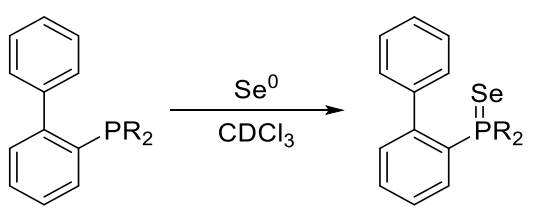

Di-tert-alkylbiarylphosphine 3 (ca $7 \mathrm{mg}$ pure, or ca $2 \mathrm{mg}$ of crude material from the examples where pure material was not obtained, vide supra) and selenium powder (20 mg, excess) were added to an NMR tube, which was evacuated and filled with anhydrous dinitrogen $(3 \times)$. Anhydrous degassed $\mathrm{CDCl}_{3}$ $(0.60 \mathrm{~mL})$ was added, and the tube was capped under a flow of anhydrous dinitrogen. After sealing with PTFE tape, the mixture was heated to $100{ }^{\circ} \mathrm{C}$ overnight. The tube was cooled to r.t., the solids were allowed to settle and a ${ }^{31} \mathrm{P}\left\{{ }^{1} \mathrm{H}\right\}$ NMR spectrum was recorded. The resulting ${ }^{31} \mathrm{P} N M R$ chemical shifts and ${ }^{1} J_{\text {P-Se }}$ coupling constants below were measured from these spectra are listed in Table S1.

\begin{tabular}{cccc}
\hline entry & $\mathbf{R}$ & $\boldsymbol{\delta}_{\mathrm{P}} / \mathbf{p p m}$ & ${ }^{\mathbf{1}} \mathbf{\mathbf { P } _ { \mathrm { PS } } \mathbf { S }} / \mathbf{H z}$ \\
\hline $\mathbf{1}$ & tert-amyl 4a & 76.71 & 732.7 \\
$\mathbf{2}$ & 3-methyl-3-pentyl 4b & 15.12 & 708.1 \\
$\mathbf{3}$ & 3-ethyl-3-pentyl 4c & 11.34 & 704.0 \\
$\mathbf{4}$ & 2,3-dimethylbut-2-yl 4d & 15.69 & 708.2 \\
$\mathbf{5}$ & 2-methyl-4-phenylbut-2-yl 4f & 73.35 & 738.8 \\
$\mathbf{6}$ & 1-methylcyclopentyl 4h & 74.69 & 723.0 \\
$\mathbf{7}$ & 1-methylcyclohexyl 4i & 80.39 & 729.3 \\
$\mathbf{8}$ & 1-adamantyl 4k & 69.99 & 726.0 \\
$\mathbf{9}$ & 2-methyladamant-2-yl 4l & 4.49 & 702.2
\end{tabular}

Table S1. ${ }^{31}$ P NMR chemical shifts $(/ p p m)$ and ${ }^{1} J_{\text {P-Se }}$ coupling constants $(/ \mathrm{Hz})$ of di-tertalkylbiarylphosphine selenides 4, measured as dilute solutions in $\mathrm{CDCl}_{3}$ at $298 \mathrm{~K}$. 


\section{Synthesis and Characterisation of Di-tert-alkylbiarylphosphine Gold(I) Chloride Complexes $\underline{5}$ (Manuscript Scheme 6)}

General procedure for the synthesis of di-tert-alkylbiarylphosphinegold(I) chloride complexes (5) (GP6)<smiles>[R20]c1ccccc1-c1ccccc1C(Cl)(Cl)Cl</smiles>

Dichloromethane $\left([3]_{0}=0.1 \mathrm{M}\right)$ was added to a vial containing di-tert-alkylbiarylphosphine 3 (50.0 $\mu \mathrm{mol}$ pure isolated material, or $0.50 \mathrm{mmol}$ crude material from the examples where pure di-tertalkylbiarylphosphine $\mathbf{3}$ was not obtained (vide infra)) and dimethylsulfide gold(I) chloride complex (1 equiv.). The mixture was stirred at r.t. for $1 \mathrm{~h}$ then concentrated in vacuo. The residue was triturated with pentane and the resulting solid was collected by Büchner filtration; where necessary, the products were further purified by passage through a pad of silica gel or Celite ${ }^{\circledR}$ (eluent: dichloromethane). The di-tert-alkylbiarylphosphinegold(I) chloride complexes 5 were obtained as offwhite solids. 
[(2-Biphenyl)di-tert-amylphosphine]gold(I) chloride (5a)

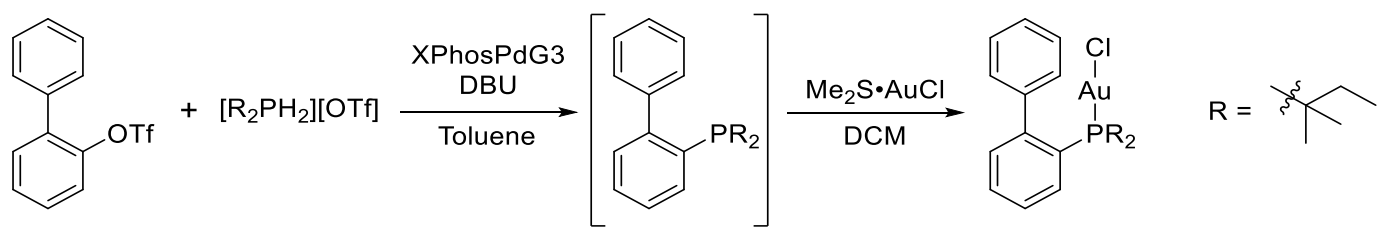

GP3 was performed using di-tert-amylphosphonium trifluoromethanesulfonate 1a $(0.138 \mathrm{~g}, 0.500$ mmol). Crude 2-(di-tert-amylphosphino)biphenyl 3a was obtained as a viscous yellow liquid $\left[{ }^{31} \mathrm{P}\left\{{ }^{1} \mathrm{H}\right\}\right.$ NMR (202 MHz, $\mathrm{CDCl}_{3}$ ): $\delta$ 14.83]. ca $2 \mathrm{mg}$ were removed for the synthesis of the selenide 4a (GP5, Section S10) and the remainder was subjected to GP6. [(2-Biphenyl)di-tert-amylphosphine]gold(I) chloride $5 a$ ( $0.146 \mathrm{~g}, 0.262 \mathrm{mmol}, 52 \%)$ was obtained as an off-white solid.

${ }^{1} \mathrm{H}$ NMR $\left(500 \mathrm{MHz}, \mathrm{CDCl}_{3}\right): \delta 7.87(\mathrm{td}, J=7.4,1.6 \mathrm{~Hz}, 1 \mathrm{H}), 7.59-7.55(\mathrm{~m}, 1 \mathrm{H}), 7.53-7.45(\mathrm{~m}, 2 \mathrm{H}), 7.44-$ $7.40(\mathrm{~m}, 2 \mathrm{H}), 7.29$ (ddd, $J=7.5,4.3,1.8 \mathrm{~Hz}, 1 \mathrm{H}), 7.14-7.10(\mathrm{~m}, 2 \mathrm{H}), 1.86(\mathrm{dt}, J=13.3,7.5 \mathrm{~Hz}, 2 \mathrm{H}) 1.78$ (dt, $J=13.5,7.6 \mathrm{~Hz}, 2 \mathrm{H}), 1.41(\mathrm{~d}, J=16.8 \mathrm{~Hz}, 6 \mathrm{H}), 1.36(\mathrm{~d}, J=15.5 \mathrm{~Hz}, 6 \mathrm{H}), 0.87(\mathrm{t}, J=7.4 \mathrm{~Hz}, 6 \mathrm{H})$.

${ }^{13} \mathrm{C}\left\{{ }^{1} \mathrm{H}\right\}$ NMR (126 MHz, $\left.\mathrm{CDCl}_{3}\right): \delta 150.5(\mathrm{~d}, J=13.1 \mathrm{~Hz}), 142.4(\mathrm{~d}, J=6.5 \mathrm{~Hz}), 134.1(\mathrm{~d}, J=2.7 \mathrm{~Hz}), 133.5$ (d, $J=7.3 \mathrm{~Hz}$ ), 130.6 (d, $J=2.3 \mathrm{~Hz}$ ), 129.3, 128.8, 128.4, 126.7 (d, $J=6.8 \mathrm{~Hz}$ ), 126.0 (d, $J=43.4 \mathrm{~Hz}$ ), 41.8 $(d, J=25.3 \mathrm{~Hz}), 34.4(\mathrm{~d}, J=8.2 \mathrm{~Hz}), 26.5(\mathrm{~d}, J=6.5 \mathrm{~Hz}), 25.5(\mathrm{~d}, J=4.0 \mathrm{~Hz}), 7.6(\mathrm{~d}, J=11.8 \mathrm{~Hz})$.

${ }^{31} \mathrm{P}\left\{{ }^{1} \mathrm{H}\right\}$ NMR (202 MHz, $\left.\mathrm{CDCl}_{3}\right): \delta 65.35(\mathrm{~s})$.

$V_{\max }($ neat $) / \mathrm{cm}^{-1}: 3048,2964,2920,2876,1462,1437,1421,1411,1386,1364,1140,1031,993,953$, $910,773,753,699,676,635,617,534,508,484$.

HRMS: calcd. for $\mathrm{C}_{22} \mathrm{H}_{31} \mathrm{AuClP}+\mathrm{Na}^{+}$: $581.1410[\mathrm{M}+\mathrm{Na}]^{+}$; found $\left(\mathrm{ESI}^{+}\right)$: 581.1404, calcd. for $\mathrm{C}_{22} \mathrm{H}_{31} \mathrm{AuClP}+\mathrm{NH}_{4}^{+}: 576.1856\left[\mathrm{M}+\mathrm{NH}_{4}\right]^{+}$; found $\left(\mathrm{ESI}^{+}\right): 576.1852$.

m.p. $/^{\circ} \mathrm{C}$ : decomposes ca $120^{\circ} \mathrm{C}$. 
[(2-Biphenyl)di-(3-methyl-3-pentyl)phosphine]gold(I) chloride (5b)

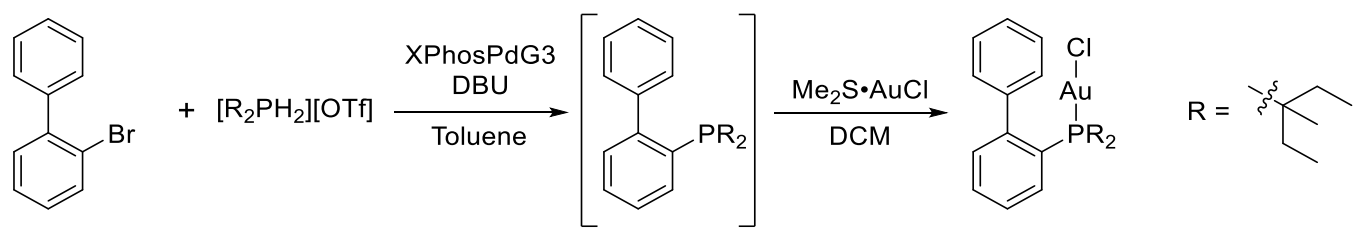

GP4 was performed using di-(3-methyl-3-pentyl)phosphonium trifluoromethanesulfonate (0.176 g, $0.500 \mathrm{mmol})$, 2-bromobiphenyl (104 $\mu \mathrm{L}, 0.600 \mathrm{mmol}), \mathrm{K}_{2} \mathrm{CO}_{3}(207 \mathrm{mg}, 1.50 \mathrm{mmol})$ and $\mathrm{Pd}\left(\mathrm{PPh}_{3}\right)_{4}(17.3$ $\mathrm{mg}, \quad 15.0 \mu \mathrm{mol}, 3 \mathrm{~mol} \%)$ in degassed xylenes $(1.0 \mathrm{~mL})$. Crude 2-(di-3-methyl-3pentylphosphino)biphenyl $\mathbf{3 b}$ was obtained as a viscous yellow liquid $\left[{ }^{31} \mathrm{P}\left\{{ }^{1} \mathrm{H}\right\} \mathbf{N M R}\left(202 \mathrm{MHz}, \mathrm{CDCl}_{3}\right)\right.$ : $\delta$ 0.05]. ca $2 \mathrm{mg}$ were removed for the synthesis of the selenide 4b (GP5, Section S10) and the remainder was subjected to GP6. [(2-Biphenyl)di-(3-methyl-3-pentyl)phosphine]gold(I) chloride 5b $(0.257 \mathrm{~g}, 0.437 \mathrm{mmol}, 87 \%)$ was obtained as an off-white solid.

Note: the signal in the ${ }^{1} \mathrm{H}$ NMR spectrum at 7.88-7.92 ppm appears as a poorly resolved td (cf. other Au complexes), the resolution is not sufficient to assign coupling constants.

${ }^{1} \mathrm{H}$ NMR $\left(500 \mathrm{MHz}, \mathrm{CDCl}_{3}\right): \delta$ 7.92-7.88 $(\mathrm{m}, 1 \mathrm{H}), 7.58-7.54(\mathrm{~m}, 1 \mathrm{H}), 7.52-7.44(\mathrm{~m}, 2 \mathrm{H}), 7.44-7.39(\mathrm{~m}$, 2H), 7.28 (ddd, $J=7.7,4.4,1.8 \mathrm{~Hz}, 1 \mathrm{H}$ ), 7.17-7.14 (m, 2H), 2.17 (ddq, $J=14.8,11.1,7.4 \mathrm{~Hz}, 2 \mathrm{H}$ ), 1.87$1.73(\mathrm{~m}, 4 \mathrm{H}), 1.72-1.61(\mathrm{~m}, 2 \mathrm{H}), 1.48(\mathrm{~d}, J=14.4 \mathrm{~Hz}, 6 \mathrm{H}), 0.95(\mathrm{t}, J=7.4 \mathrm{~Hz}, 6 \mathrm{H}), 0.89(\mathrm{t}, J=7.4 \mathrm{~Hz}, 6 \mathrm{H})$. ${ }^{13} \mathrm{C}\left\{{ }^{1} \mathrm{H}\right\}$ NMR $\left(126 \mathrm{MHz}, \mathrm{CDCl}_{3}\right): \delta 150.0(\mathrm{~d}, J=13.4 \mathrm{~Hz}), 142.5(\mathrm{~d}, J=6.2 \mathrm{~Hz}), 135.1(\mathrm{~d}, J=2.7 \mathrm{~Hz}), 134.0$ (d, J = 7.5 Hz), 130.5 (d, J = 2.6 Hz), 129.3, 128.7, 128.4, 126.6 (d, J = 41.0 Hz), 126.5 (d, J = 6.7 Hz), 45.5 (d, $J=23.6), 32.8(\mathrm{~d}, J=10.3 \mathrm{~Hz}), 31.3(\mathrm{~d}, J=5.2 \mathrm{~Hz}), 24.1(\mathrm{~d}, J=1.9 \mathrm{~Hz}), 9.5(\mathrm{~d}, J=10.4 \mathrm{~Hz}), 9.3(\mathrm{~d}, J=$ $8.7 \mathrm{~Hz})$.

${ }^{31} \mathrm{P}\left\{{ }^{1} \mathrm{H}\right\} \mathrm{NMR}\left(202 \mathrm{MHz}, \mathrm{CDCl}_{3}\right): \delta 58.34(\mathrm{~s})$.

$v_{\max }($ neat $) / \mathrm{cm}^{-1}: 2967,2936,2876,1460,1441,1384,1121,1062,1033,1006,779,750,698,615,532$, $506,495,457$.

HRMS: calcd. for $\mathrm{C}_{24} \mathrm{H}_{35} \mathrm{AuClP}+\mathrm{Na}^{+}$: $609.1723[\mathrm{M}+\mathrm{Na}]^{+}$; found $\left.(\mathrm{ESI})^{+}\right): 609.1718$, calcd. for $\mathrm{C}_{24} \mathrm{H}_{35} \mathrm{AuClP}+\mathrm{NH}_{4}^{+}: 604.2169\left[\mathrm{M}+\mathrm{NH}_{4}\right]^{+}$; found $\left(\mathrm{ESI}^{+}\right): 604.2162$.

m.p. $/{ }^{\circ} \mathrm{C}$ : decomposes ca $130^{\circ} \mathrm{C}$. 
[(2-Biphenyl)di-(3-ethyl-3-pentyl)phosphine]gold(I) chloride (5c)

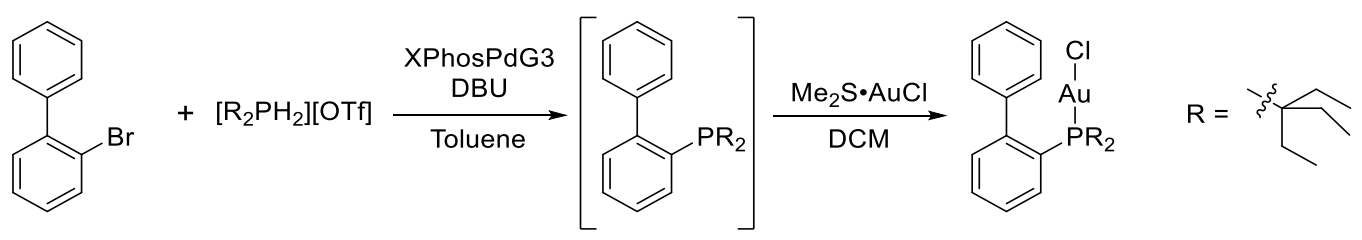

GP4 was performed using di-(3-ethyl-3-pentyl)phosphonium trifluoromethanesulfonate 1c (0.190 g, $0.500 \mathrm{mmol}), 2$-bromobiphenyl $(104 \mu \mathrm{L}, 0.600 \mathrm{mmol}), \mathrm{K}_{2} \mathrm{CO}_{3}(207 \mathrm{mg}, 1.50 \mathrm{mmol})$ and $\mathrm{Pd}\left(\mathrm{PPh}_{3}\right)_{4}(17.3$ $\mathrm{mg}, 15.0 \mu \mathrm{mol}, 3 \mathrm{~mol} \%)$ in degassed xylenes $(1.0 \mathrm{~mL}$ ). Crude 2-(di-3-ethyl-3-pentylphosphino)biphenyl was obtained as a viscous yellow liquid [ $\left.{ }^{31} \mathrm{P}\left\{{ }^{1} \mathrm{H}\right\} \mathrm{NMR}\left(202 \mathrm{MHz}, \mathrm{CDCl}_{3}\right): \delta 5.16\right]$. $\mathrm{ca} 2 \mathrm{mg}$ were removed for the synthesis of the selenide 4c (GP5, Section S10) and the remainder was subjected to GP6. [(2Biphenyl)di-(3-ethyl-3-pentyl)phosphine]gold(I) chloride 5c (0.166 g, $0.270 \mathrm{mmol}, 54 \%)$ was obtained as an off-white solid.

${ }^{1} \mathrm{H}$ NMR $\left(500 \mathrm{MHz}, \mathrm{CDCl}_{3}\right.$ ): $\delta 7.94$ (app. t, $J=7.6 \mathrm{~Hz}, 1 \mathrm{H}$ ), 7.56 (app. t, $\left.J=7.6 \mathrm{~Hz}, 1 \mathrm{H}\right), 7.51-7.38(\mathrm{~m}, 4 \mathrm{H})$, 7.28-7.24 (m, $1 \mathrm{H}$, overlap with residual $\mathrm{CHCl}_{3}$ peak), 7.18 (app. $\mathrm{d}, J=7.5 \mathrm{~Hz}, 2 \mathrm{H}$ ), 2.12 (ddq, $J=14.8$, $14.8,7.4 \mathrm{~Hz}, 6 \mathrm{H}$ ), 1.97 (ddq, $J=14.8,14.8,7.4 \mathrm{~Hz}, 6 \mathrm{H}), 0.99(\mathrm{t}, J=7.4 \mathrm{~Hz}, 18 \mathrm{H})$.

${ }^{13} \mathrm{C}\left\{{ }^{1} \mathrm{H}\right\}$ NMR $\left(126 \mathrm{MHz}, \mathrm{CDCl}_{3}\right): \delta 149.5(\mathrm{~d}, J=13.2 \mathrm{~Hz}), 142.8(\mathrm{~d}, J=6.0 \mathrm{~Hz}), 135.6(\mathrm{~d}, J=2.7 \mathrm{~Hz}), 134.3$ (d, J = 7.4 Hz), 130.4 (d, J = 2.4 Hz), 129.3, 128.7, 128.4, 127.8 (d, J = 37.2 Hz), 126.4 (d, J = 6.4 Hz), 50.7 (d, $J=19.7 \mathrm{~Hz}), 30.3(\mathrm{~d}, J=6.0 \mathrm{~Hz}), 10.5(\mathrm{~d}, J=8.2 \mathrm{~Hz})$.

${ }^{31} \mathrm{P}\left\{{ }^{1} \mathrm{H}\right\}$ NMR $\left(202 \mathrm{MHz}, \mathrm{CDCl}_{3}\right): \delta 59.91(\mathrm{~s})$.

$v_{\max }($ neat $) / \mathrm{cm}^{-1}:$ 2966, 2939, 2879, 1583, 1457, 1439, 1383, 1335, 1154, 1122, 1069, 1033, 1006, 909, $839,777,750,699,671,615,547,526,507,493,465$.

HRMS: calcd. for $\mathrm{C}_{26} \mathrm{H}_{39} \mathrm{AuClP}+\mathrm{Na}^{+}$: $637.2036[\mathrm{M}+\mathrm{Na}]^{+}$; found $\left.(\mathrm{ESI})^{+}\right)$: 637.2022, calcd. for $\mathrm{C}_{26} \mathrm{H}_{39} \mathrm{AuClP}+\mathrm{NH}_{4}^{+}: 632.2482\left[\mathrm{M}+\mathrm{NH}_{4}\right]^{+}$; found $\left(\mathrm{ESI}{ }^{+}\right): 632.2465$.

m.p. $/{ }^{\circ} \mathrm{C}: 167-169$. 
[(2-Biphenyl)bis-(2,3-dimethyl-2-butyl)phosphine]gold(I) chloride (5d)

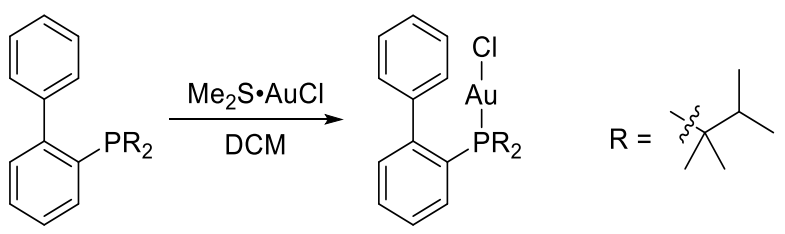

GP6 was performed using 2-(bis-(2,3-dimethyl-2-butyl)phosphino)biphenyl 3d (17.7 mg, $50.0 \mu \mathrm{mol}$ ). [(2-Biphenyl)bis-(2,3-dimethyl-2-butyl)phosphine]gold(I) chloride 5d (19.7 mg, $33.6 \mu \mathrm{mol}, 67 \%)$ was obtained as an off-white solid.

${ }^{1} \mathrm{H}$ NMR $\left(500 \mathrm{MHz}, \mathrm{CDCl}_{3}\right): \delta 7.81(\mathrm{~d}, J=7.5,1.5 \mathrm{~Hz}, 1 \mathrm{H}), 7.58-7.54(\mathrm{~m}, 1 \mathrm{H}), 7.51(\mathrm{tt}, J=7.4 .1 .6 \mathrm{~Hz}, 1 \mathrm{H})$, $7.46(\mathrm{tt}, J=7.4,1.5 \mathrm{~Hz}, 1 \mathrm{H}), 7.44-7.39(\mathrm{~m}, 2 \mathrm{H}), 7.29$ (ddd, $J=7.6,4.4,1.7 \mathrm{~Hz}, 1 \mathrm{H}), 7.20-7.17(\mathrm{~m}, 2 \mathrm{H})$, $2.17(\mathrm{dh}, J=10.7,6.8 \mathrm{~Hz}, 2 \mathrm{H}), 1.47(\mathrm{~d}, J=13.8 \mathrm{~Hz}, 6 \mathrm{H}), 1.45(\mathrm{~d}, J=18.4 \mathrm{~Hz}, 6 \mathrm{H}) 0.97(\mathrm{~d}, J=6.7 \mathrm{~Hz}, 6 \mathrm{H})$, $0.89(\mathrm{~d}, J=6.8 \mathrm{~Hz}, 6 \mathrm{H})$.

${ }^{13} \mathrm{C}\left\{{ }^{1} \mathrm{H}\right\}$ NMR $\left(126 \mathrm{MHz}, \mathrm{CDCl}_{3}\right): \delta 149.6(\mathrm{~d}, J=13.1 \mathrm{~Hz}), 142.2(\mathrm{~d}, J=6.0 \mathrm{~Hz}), 135.3(\mathrm{~d}, J=2.6 \mathrm{~Hz}), 134.2$ (d, $J=7.4 \mathrm{~Hz}$ ), 130.6 (d, J = 2.5 Hz), 129.3, 128.7, 128.4, 127.0 (d, $J=40.1 \mathrm{~Hz}$ ), 126.5 (d, J = 6.7 Hz), 45.7 (d, J = 23.2 Hz), 35.1 (d, J = 4.9 Hz), 26.4 (d, J = 9.4 Hz), 23.9, 19.5 (d, J= 7.8 Hz), 19.1 (d, J = 5.1 Hz).

${ }^{31} \mathrm{P}\left\{{ }^{1} \mathrm{H}\right\}$ NMR (202 MHz, $\left.\mathrm{CDCl}_{3}\right): \delta 58.33(\mathrm{~s})$.

$v_{\text {max }}($ neat $) / \mathrm{cm}^{-1}:$ 2960, 2874, 1462, 1442, 1392, 1367, 1237, 1156, 1125, 1083, 1007, 908, 780, 756, $699,678,616,541,511,465,416$.

HRMS: calcd. for $\mathrm{C}_{24} \mathrm{H}_{35} \mathrm{AuClP}+\mathrm{Na}^{+}$: $609.1723[\mathrm{M}+\mathrm{Na}]^{+}$; found $\left.(\mathrm{ESI})^{+}\right)$: 609.1717, calcd. for $\mathrm{C}_{24} \mathrm{H}_{35} \mathrm{AuClP}+\mathrm{NH}_{4}{ }^{+}: 604.2169\left[\mathrm{M}+\mathrm{NH}_{4}\right]^{+}$; found $\left(\mathrm{ESI}^{+}\right): 604.2164$.

m.p./ ${ }^{\circ} \mathrm{C}: 172-174$. 
[(2-Biphenyl)di-(2-methyl-4-phenyl-2-butyl)phosphine]gold(I) chloride (5f)<smiles>[R]CC(C)(C)CCc1ccccc1</smiles>

GP6 was performed using 2-(di-(2-methyl-4-phenyl-2-butyl)phosphino)biphenyl 3f (23.9 mg, 50.0 umol). [(2-Biphenyl)di-(2-methyl-4-phenyl-2-butyl)phosphine]gold(I) chloride $5 f(24.3 \mathrm{mg}, 34.2$ umol, $68 \%)$ was obtained as an off-white solid.

${ }^{1} \mathrm{H}$ NMR $\left(500 \mathrm{MHz}, \mathrm{CDCl}_{3}\right): \delta 7.87(\mathrm{td}, J=7.6,1.4 \mathrm{~Hz}, 1 \mathrm{H}), 7.57-7.51(\mathrm{~m}, 2 \mathrm{H}), 7.48(\mathrm{tt}, J=7.4,1.5 \mathrm{~Hz}$, $1 \mathrm{H})$, 7.38-7.33 (m, 2H), 7.31-7.24 ( $\mathrm{m}, 5 \mathrm{H}$, overlap with residual $\mathrm{CHCl}_{3}$ peak), 7.20-7.16 $(\mathrm{m}, 2 \mathrm{H}), 7.04$ (dd, $J=7.7,1.5 \mathrm{~Hz}, 6 \mathrm{H}), 2.69-2.56(\mathrm{~m}, 4 \mathrm{H}), 2.14-1.97(\mathrm{~m}, 4 \mathrm{H}), 1.55(\mathrm{~d}, J=16.9 \mathrm{~Hz}, 6 \mathrm{H}), 1.54(\mathrm{~d}, J=15.3$ $\mathrm{Hz}, 6 \mathrm{H})$.

${ }^{13} \mathrm{C}\left\{{ }^{1} \mathrm{H}\right\}$ NMR (126 MHz, CDCl $): \delta 150.5(\mathrm{~d}, J=13.4 \mathrm{~Hz}), 142.1(\mathrm{~d}, J=6.3 \mathrm{~Hz}), 141.5,134.2(\mathrm{~d}, J=2.5$ $\mathrm{Hz}), 133.8(\mathrm{~d}, J=7.4 \mathrm{~Hz}), 130.9$ (d, $J=2.4 \mathrm{~Hz}), 129.3,128.8,128.7,128.5,128.4,126.9$ (d, $J=6.7 \mathrm{~Hz})$, 126.3, 125.5 (d, J = 43.3 Hz), 43.8 (d, J = 6.7 Hz), 41.9 (d, J = 24.2 Hz), 29.8 (d, J = 10.4 Hz), 28.1 (d, J = $7.6 \mathrm{~Hz}), 26.9(\mathrm{~d}, J=3.7 \mathrm{~Hz})$.

${ }^{31} \mathrm{P}\left\{{ }^{1} \mathrm{H}\right\}$ NMR (202 MHz, $\left.\mathrm{CDCl}_{3}\right): \delta 63.55$ (s).

$v_{\max }($ neat $) / \mathrm{cm}^{-1}: 3060,3024,2965,2926,2853,1601,1496,1453,1386,1365,1200,1130,1071,1008$, $906,776,745,717,697,617,573,531,511,414$.

HRMS: calcd. for $\mathrm{C}_{34} \mathrm{H}_{39} \mathrm{AuClP}+\mathrm{Na}^{+}$: $733.2036[\mathrm{M}+\mathrm{Na}]^{+}$; found $\left(\mathrm{ESI}^{+}\right)$: 733.2015, calcd. for $\mathrm{C}_{34} \mathrm{H}_{39} \mathrm{AuClP}+\mathrm{NH}_{4}^{+}: 728.2482\left[\mathrm{M}+\mathrm{NH}_{4}\right]^{+}$; found $\left(\mathrm{ESI}^{+}\right):$: 728.2467.

m.p./ ${ }^{\circ} \mathrm{C}: 130-132$. 
[(2-Biphenyl)di-(1-methylcyclopentyl)phosphine]gold(I) chloride (5h)<smiles>[R]C(=O)C1(CC)CCCC1</smiles>

GP6 was performed using 2-(di-(1-methylcyclopentyl)phosphino)biphenyl 3h (17.5 mg, $50.0 \mu \mathrm{mol})$. [(2-Biphenyl)di-(1-methylcyclopentyl)phosphine]gold(I) chloride 5 h $(21.9 \mathrm{mg}, 37.6 \mu \mathrm{mol}, 75 \%)$ was obtained as an off-white solid.

${ }^{1} \mathrm{H}$ NMR $\left(500 \mathrm{MHz}, \mathrm{CDCl}_{3}\right): \delta 7.90(\mathrm{td}, J=7.6,1.6 \mathrm{~Hz}, 1 \mathrm{H}), 7.58-7.54(\mathrm{~m}, 1 \mathrm{H}), 7.52-7.40(\mathrm{~m}, 4 \mathrm{H}), 7.29$ (ddd, $J=7.5,4.3,1.7 \mathrm{~Hz}, 1 \mathrm{H}), 7.18-7.15(\mathrm{~m}, 2 \mathrm{H}), 2.30-2.20(\mathrm{~m}, 2 \mathrm{H}), 2.09-1.99(\mathrm{~m}, 2 \mathrm{H}), 1.83-1.70(\mathrm{~m}$, $6 \mathrm{H}), 1.69-1.56(\mathrm{~m}, 6 \mathrm{H}), 1.37(\mathrm{~d}, J=14.9 \mathrm{~Hz}, 6 \mathrm{H})$.

${ }^{13} \mathrm{C}\left\{{ }^{1} \mathrm{H}\right\}$ NMR (126 MHz, $\left.\mathrm{CDCl}_{3}\right): \delta 150.0(\mathrm{~d}, J=13.5 \mathrm{~Hz}), 142.3(\mathrm{~d}, J=6.3 \mathrm{~Hz}), 134.2(\mathrm{~d}, J=2.8 \mathrm{~Hz}), 133.3$ (d, J = 7.4 Hz), 130.5 (d, J = 2.4 Hz), 129.5, 128.9, 128.3, 126.8 (d, J=6.9 Hz), 126.8 (d, J = 46.5 Hz), 45.9 (d, J = 28.6 Hz), 40.6 (d, J = 9.3 Hz), $40.4(\mathrm{~d}, J=8.4 \mathrm{~Hz}), 25.9$ (d, J = 4.9 Hz), 24.3 (d, J = 9.8 Hz), 23.4 (d, $J=10.2 \mathrm{~Hz})$.

${ }^{31} \mathrm{P}\left\{{ }^{1} \mathrm{H}\right\}$ NMR (202 MHz, $\left.\mathrm{CDCl}_{3}\right): \delta 58.89(\mathrm{~s})$.

$V_{\max }\left(\right.$ neat) $/ \mathrm{cm}^{-1}:$ 2954, 2867, 1463, 1441, 1424, 1376, 778, 754, 702, 679, 616, 537, 523, 511.

HRMS: calcd. for $\mathrm{C}_{24} \mathrm{H}_{31} \mathrm{AuClP}+\mathrm{Na}^{+}$: $605.1410[\mathrm{M}+\mathrm{Na}]^{+}$; found $\left(\mathrm{ESI}^{+}\right)$: 605.1406, calcd. for $\mathrm{C}_{24} \mathrm{H}_{31} \mathrm{AuClP}+\mathrm{NH}_{4}^{+}: 600.1856\left[\mathrm{M}+\mathrm{NH}_{4}\right]^{+}$; found $\left(\mathrm{ESI}^{+}\right): 600.1852$.

m.p. $/{ }^{\circ} \mathrm{C}: 160-163$. 
[(2-Biphenyl)di-(1-methylcyclohexyl)phosphine]gold(I) chloride (5i)

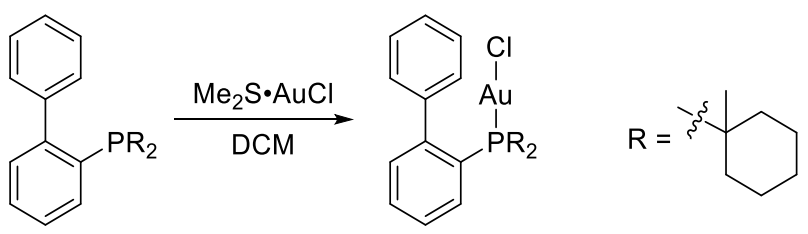

GP6 was performed using 2-(di-(1-methylcyclohexyl)phosphino)biphenyl 3i (18.9 mg, $50.0 \mu \mathrm{mol})$. [(2-Biphenyl)di-(1-methylcyclohexyl)phosphine]gold(I) chloride $5 \mathbf{i}$ (24.8 mg, $40.6 \mu \mathrm{mol}, 81 \%)$ was obtained as an off-white solid.

${ }^{1} \mathrm{H}$ NMR $\left(500 \mathrm{MHz}, \mathrm{CDCl}_{3}\right): \delta 7.90(\mathrm{td}, J=7.1,1.7 \mathrm{~Hz}, 1 \mathrm{H}), 7.60-7.55(\mathrm{~m}, 1 \mathrm{H}), 7.52-7.44(\mathrm{~m}, 2 \mathrm{H})$, 7.44$7.40(\mathrm{~m}, 2 \mathrm{H}), 7.30-7.27$ (ddd, $J=7.4,2.4,1.8 \mathrm{~Hz}, 1 \mathrm{H}), 7.14-7.10(\mathrm{~m}, 2 \mathrm{H}), 2.13-2.04(\mathrm{~m}, 2 \mathrm{H}), 2.03-1.88$ $(\mathrm{m}, 4 \mathrm{H}), 1.79-1.72(\mathrm{~m}, 2 \mathrm{H}), 1.65-1.43(\mathrm{~m}, 16 \mathrm{H}), 1.18-1.08(\mathrm{~m}, 2 \mathrm{H})$.

${ }^{13} \mathrm{C}\left\{{ }^{1} \mathrm{H}\right\}$ NMR $\left(126 \mathrm{MHz}, \mathrm{CDCl}_{3}\right): \delta 150.8(\mathrm{~d}, J=13.0 \mathrm{~Hz}), 142.5(\mathrm{~d}, J=6.5 \mathrm{~Hz}), 134.5(\mathrm{~d}, J=2.4 \mathrm{~Hz}), 133.6$ (d, J = 7.2 Hz), 130.4 (d, J = 2.3 Hz), 129.4, 128.8, 128.3, 126.5 (d, J = 6.4 Hz), $125.2(\mathrm{~d}, J=42.2 \mathrm{~Hz}), 43.2$ (d, $J=24.2 \mathrm{~Hz}$ ), 37.6, $37.5(\mathrm{~d}, J=1.8 \mathrm{~Hz}), 25.5(\mathrm{~d}, J=1.3 \mathrm{~Hz}), 21.7(\mathrm{~d}, J=4.0 \mathrm{~Hz}), 21.3(\mathrm{~d}, J=7.8 \mathrm{~Hz})$, $21.3(\mathrm{~d}, J=7.3 \mathrm{~Hz})$.

${ }^{31} \mathrm{P}\left\{{ }^{1} \mathrm{H}\right\}$ NMR (202 MHz, $\left.\mathrm{CDCl}_{3}\right): \delta 71.19(\mathrm{~s})$.

$v_{\max }($ neat $) / \mathrm{cm}^{-1}:$ 2934, 2866, 2844, 1463, 1441, 1427, 1374, 1345, 1333, 1272, 1255, 1239, 1159, 1146, 1131, 1081, 1030, 1007, 972, 933, 899, 851, 775, 752, 693, 648, 616, 552, 528, 513, 497, 462, 436.

HRMS: calcd. for $\mathrm{C}_{26} \mathrm{H}_{35} \mathrm{AuClP}+\mathrm{Na}^{+}$: $633.1723\left[\mathrm{M}+\mathrm{Na}^{+}\right]$; found $\left(\mathrm{ESI}^{+}\right): 633.1717$, calcd. for $\mathrm{C}_{26} \mathrm{H}_{35} \mathrm{AuClP}+\mathrm{NH}_{4}^{+}: 628.2169\left[\mathrm{M}+\mathrm{NH}_{4}\right]^{+}$; found $\left(\mathrm{ESI}^{+}\right): 628.2159$.

m.p. $/{ }^{\circ} \mathrm{C}$ : decomposes ca $220^{\circ} \mathrm{C}$. 
[(2-Biphenyl)di-(1-adamantyl)phosphine]gold(I) chloride (5k)

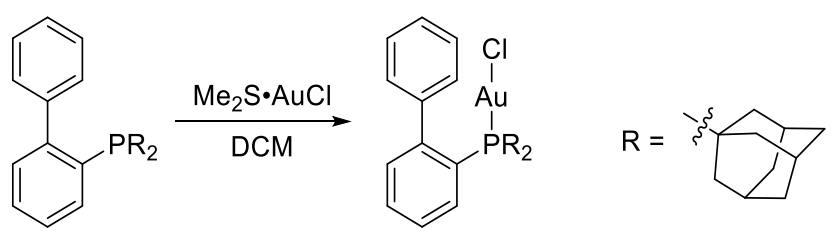

GP6 was performed using 2-(di-(1-adamantyl)phosphino)biphenyl 3k (22.7 mg, $50.0 \mu \mathrm{mol})$. [(2-Biphenyl)di-(1-adamantyl)phosphine]gold(I) chloride 5k (23.7 mg, $34.5 \mu \mathrm{mol}, 69 \%)$ was obtained as an off-white solid. The synthesis ${ }^{24}$ and crystal structure ${ }^{25}$ for $\mathbf{5 k}$ have been reported previously by Stradiotto and co-workers.

${ }^{1} \mathrm{H}$ NMR $\left(500 \mathrm{MHz}, \mathrm{CDCl}_{3}\right): \delta 7.86(\mathrm{td}, J=7.0,2.0 \mathrm{~Hz}, 1 \mathrm{H}), 7.59-7.55(\mathrm{~m}, 1 \mathrm{H}), 7.53-7.47(\mathrm{~m}, 2 \mathrm{H}), 7.45-$ $7.41(\mathrm{~m}, 2 \mathrm{H}), 7.30$ (ddd, $J=7.0,4.3,2.0 \mathrm{~Hz}, 1 \mathrm{H}), 7.13-7.09(\mathrm{~m}, 2 \mathrm{H}), 2.22-2.08(\mathrm{~m}, 12 \mathrm{H}), 2.01-1.97(\mathrm{~m}$, $6 \mathrm{H}), 1.70-1.65(\mathrm{~m}, 12 \mathrm{H})$.

${ }^{13} \mathrm{C}\left\{{ }^{1} \mathrm{H}\right\}$ NMR (126 MHz, CDCl 3 ): $\delta 150.9(\mathrm{~d}, J=13.1 \mathrm{~Hz}), 142.5(\mathrm{~d}, J=6.4 \mathrm{~Hz}), 134.5(\mathrm{~d}, J=2.4 \mathrm{~Hz}), 133.6$ (d, $J=7.3 \mathrm{~Hz}$ ), 130.5 (d, J = 2.3 Hz), 129.4, 128.7, 128.3, 126.4 (d, J = 6.5 Hz), 124.0 (d, J = 43.7 Hz), 42.7 (d, $J=23.6 \mathrm{~Hz}), 42.3(\mathrm{~d}, J=2.7 \mathrm{~Hz}), 36.4(\mathrm{~d}, J=1.6 \mathrm{~Hz}), 28.7$ (d, $J=9.9 \mathrm{~Hz})$.

${ }^{31} \mathrm{P}\left\{{ }^{1} \mathrm{H}\right\}$ NMR $\left(202 \mathrm{MHz}, \mathrm{CDCl}_{3}\right): \delta 61.57(\mathrm{~s})$.

$v_{\max }($ neat $) / \mathrm{cm}^{-1}:$ 3046, 2901, 2848, 1465, 1449, 1343, 1301, 1275, 1259, 1127, 1044, 969, 905, 773, $752,730,694,547,531,517,488,453,433$.

HRMS: calcd. for $\mathrm{C}_{32} \mathrm{H}_{39} \mathrm{AuClP}+\mathrm{Na}^{+}$: $709.2036[\mathrm{M}+\mathrm{Na}]^{+}$; found $\left(\mathrm{ESI}^{+}\right)$: 709.2029, calcd. for $\mathrm{C}_{32} \mathrm{H}_{39} \mathrm{AuClP}+\mathrm{NH}_{4}^{+}: 704.2482\left[\mathrm{M}+\mathrm{NH}_{4}\right]^{+}$; found $\left(\mathrm{ESI}^{+}\right): 704.2448$.

m.p. $/^{\circ} \mathrm{C}:>270$. 
[(2-Biphenyl)di-(2-methyl-2-adamantyl)phosphine]gold(I) chloride (5I)

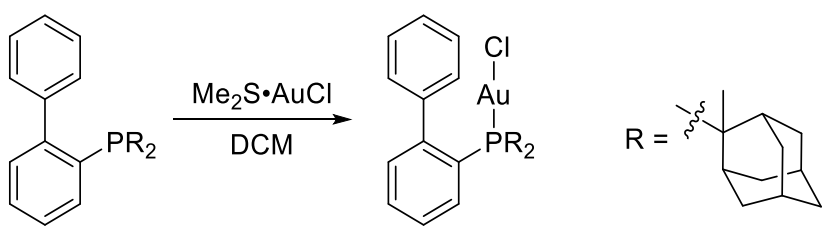

GP6 was followed using 2-(di-(2-methyl-2-adamantyl)phosphino)biphenyl 3l (12.1 mg, $25.0 \mu \mathrm{mol}$ ) and dimethylsulfide gold(I) chloride complex $(7.4 \mathrm{mg}, 25$ umol). [(2-Biphenyl)di-(2-methyl-2adamantyl)phosphine]gold(I) chloride $5 \mathrm{l}(6.0 \mathrm{mg}, 8.4 \mu \mathrm{mol}, 34 \%)$ was obtained as an off-white solid.

The ${ }^{1} \mathrm{H}$ and ${ }^{31} \mathrm{P}$ NMR spectra of this compound are complicated by restricted rotation. Variable temperature ${ }^{31} \mathrm{P}$ NMR spectra (DMSO- $d_{6}, 298-368 \mathrm{~K}$ ) showed four different signals for the complex which tended towards coalescence at higher temperatures (Figure S9).

HRMS: calcd. for $\mathrm{C}_{34} \mathrm{H}_{43} \mathrm{AuClP}+\mathrm{Na}^{+}: 737.2349[\mathrm{M}+\mathrm{Na}]^{+}$; found $\left.(\mathrm{ESI})^{+}\right): 737.2344$.

$368 \mathrm{~K}$

$358 \mathrm{~K}$

$348 \mathrm{~K}$

$338 \mathrm{~K}$

$328 \mathrm{~K}$

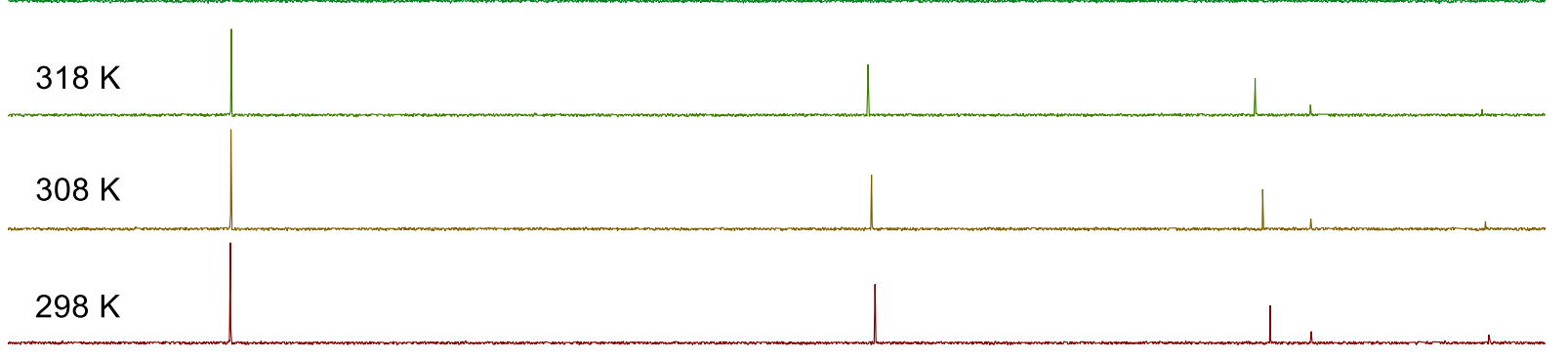

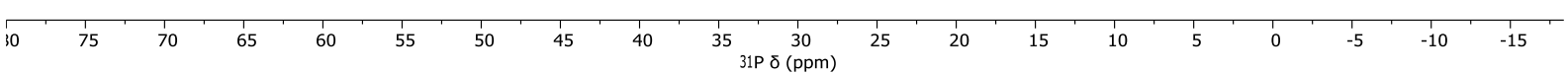

Figure S9. Variable temperature ${ }^{31} \mathrm{P}$ NMR spectra of $\mathbf{5 l}$ in DMSO- $d_{6}$. 


\section{Suzuki-Miyaura Chemoselectivity Study (Manuscript Scheme 7)}

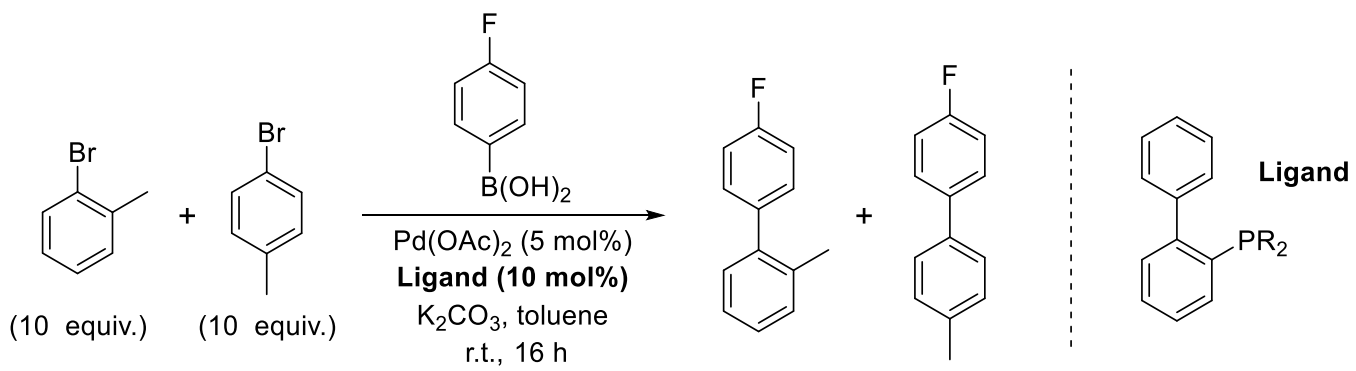

Bromotoluene stock solution: 4-bromotoluene $(3.42 \mathrm{~g}, 20.0 \mathrm{mmol})$ and 2-bromotoluene $(3.42 \mathrm{~g}, 20.0$ mmol) were added to a dry Schlenk tube that had previously been evacuated and backfilled with anhydrous dinitrogen $(3 \times)$, and the mixture was made up to $20.0 \mathrm{~mL}$ total volume with anhydrous degassed toluene.

Competition reactions: 4-fluorophenylboronic acid ( $28.0 \mathrm{mg}, 0.200 \mathrm{mmol}), \mathrm{K}_{2} \mathrm{CO}_{3}(0.276 \mathrm{~g}, 2.00 \mathrm{mmol})$ $\mathrm{Pd}(\mathrm{OAc})_{2}(2.25 \mathrm{mg}, 10.0 \mu \mathrm{mol}, 5 \mathrm{~mol} \%)$ and ligand (3d, 3f, 3h, 3i, 3k, 3l, or $\left.\mathbf{3 0} ; 20.0 \mu \mathrm{mol}, 10 \mathrm{~mol} \%\right)$ were added to microwave tubes. The tubes were capped, then evacuated and backfilled with anhydrous dinitrogen $(3 \times)$. Bromotoluene stock solution $(2.0 \mathrm{~mL})$ was added to each tube, and the mixtures were stirred at r.t. for $16 \mathrm{~h}$. An aliquot of each reaction was removed, diluted with $\mathrm{CDCl}_{3}$, and the ratio of products was measured by ${ }^{19} \mathrm{~F}$ NMR spectroscopy. Four peaks were observed in all cases, the identity of which were confirmed by comparison to authentic samples (Figure S10).

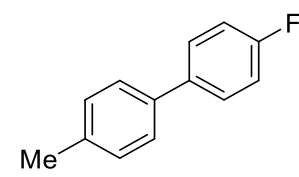

$\delta_{\mathrm{F}}=-115.8 \mathrm{ppm}$

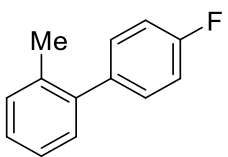

$\delta_{\mathrm{F}}=-115.6 \mathrm{ppm}$

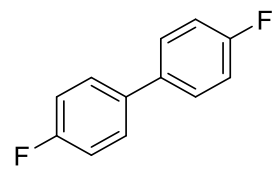

$\delta_{\mathrm{F}}=-115.2 \mathrm{ppm}$

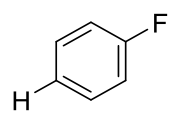

$\delta_{\mathrm{F}}=-112.5 \mathrm{ppm}$

Figure S10. ${ }^{19} \mathrm{~F}$ NMR chemical shifts $\left(/ \mathrm{ppm}, \mathrm{CDCl}_{3}\right)$ of species observed in Suzuki-Miyaura crosscouplings. 


\section{X-ray Crystallography Data Tables}

Table S2. Crystal data and structure refinement for 1a (CCDC 1990404; triflate anion and $\mathrm{H}$ atoms on Comitted for clarity).

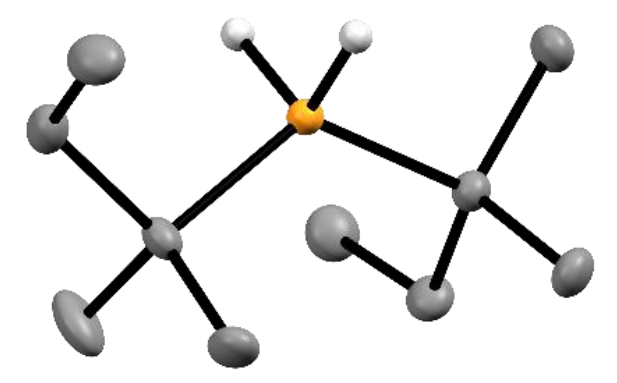

\begin{tabular}{|c|c|}
\hline Empirical formula & $\mathrm{C}_{11} \mathrm{H}_{24} \mathrm{O}_{3} \mathrm{~F}_{3} \mathrm{PS}$ \\
\hline Formula weight & 324.33 \\
\hline Temperature/K & $120(2)$ \\
\hline Crystal system & monoclinic \\
\hline Space group & $\mathrm{P} 2{ }_{1} / \mathrm{n}$ \\
\hline $\mathrm{a} / \AA ̊$ & $17.4780(3)$ \\
\hline b/Å & $10.4898(2)$ \\
\hline$c / \AA ̊$ & $17.7113(4)$ \\
\hline$\alpha /^{\circ}$ & 90.0 \\
\hline$\beta /^{\circ}$ & $99.9348(18)$ \\
\hline$\gamma /{ }^{\circ}$ & 90.0 \\
\hline Volume $/ \AA^{3}$ & $3198.51(11)$ \\
\hline Z & 8 \\
\hline$\rho_{\text {calc }} / \mathrm{cm}^{3}$ & 1.347 \\
\hline$\mu / \mathrm{mm}^{-1}$ & 3.067 \\
\hline$F(000)$ & 1376.0 \\
\hline Crystal size $/ \mathrm{mm}^{3}$ & $0.232 \times 0.166 \times 0.077$ \\
\hline Radiation & $\operatorname{CuK} \alpha(\lambda=1.54184)$ \\
\hline $2 \Theta$ range for data collection $/^{\circ}$ & 6.562 to 147.572 \\
\hline Index ranges & $-21 \leq h \leq 18,-12 \leq k \leq 9,-19 \leq \mathrm{I} \leq 21$ \\
\hline Reflections collected & 11217 \\
\hline Independent reflections & $6130\left[R_{\text {int }}=0.0244, R_{\text {sigma }}=0.0319\right]$ \\
\hline Data/restraints/parameters & $6130 / 0 / 371$ \\
\hline Goodness-of-fit on $\mathrm{F}^{2}$ & 1.020 \\
\hline Final $R$ indexes $[I>=2 \sigma(I)]$ & $\mathrm{R}_{1}=0.0326, \mathrm{wR}_{2}=0.0852$ \\
\hline Final $R$ indexes [all data] & $\mathrm{R}_{1}=0.0388, w \mathrm{w}_{2}=0.0902$ \\
\hline Largest diff. peak/hole / e $\AA^{-3}$ & $0.39 /-0.28$ \\
\hline
\end{tabular}


Table S3. Crystal data and structure refinement for 1d (CCDC 1990405; triflate anion and H atoms on Comitted for clarity).

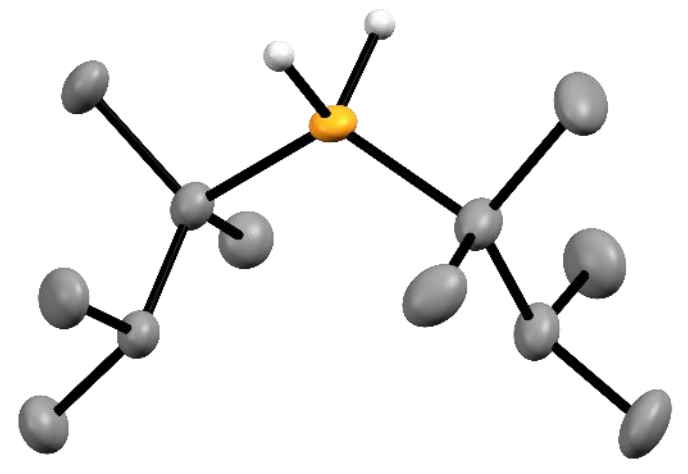

\begin{tabular}{|c|c|}
\hline Empirical formula & $\mathrm{C}_{13} \mathrm{H}_{28} \mathrm{~F}_{3} \mathrm{O}_{3} \mathrm{PS}$ \\
\hline Formula weight & 352.38 \\
\hline Temperature/K & $120(2)$ \\
\hline Crystal system & monoclinic \\
\hline Space group & $\mathrm{P} 2{ }_{1} / \mathrm{n}$ \\
\hline$a / \AA ̊$ & $8.8317(4)$ \\
\hline $\mathrm{b} / \AA ̊ \AA$ & $22.6453(9)$ \\
\hline$c / \AA ̊$ & $9.2451(6)$ \\
\hline$\alpha /^{\circ}$ & 90 \\
\hline$\beta /^{\circ}$ & $106.967(6)$ \\
\hline$\gamma /{ }^{\circ}$ & 90 \\
\hline Volume $/ \AA^{3}$ & $1768.51(17)$ \\
\hline Z & 4 \\
\hline$\rho_{\text {calc }} \mathrm{g} / \mathrm{cm}^{3}$ & 1.323 \\
\hline$\mu / \mathrm{mm}^{-1}$ & 2.814 \\
\hline$F(000)$ & 752.0 \\
\hline Crystal size $/ \mathrm{mm}^{3}$ & $0.326 \times 0.242 \times 0.142$ \\
\hline Radiation & $\operatorname{CuK} \alpha(\lambda=1.54184)$ \\
\hline $2 \Theta$ range for data collection $/^{\circ}$ & 10.74 to 147.248 \\
\hline Index ranges & $-7 \leq h \leq 10,-26 \leq k \leq 27,-11 \leq \mathrm{I} \leq 7$ \\
\hline Reflections collected & 6294 \\
\hline Independent reflections & $3407\left[R_{\text {int }}=0.0431, R_{\text {sigma }}=0.0363\right]$ \\
\hline Data/restraints/parameters & $3407 / 1050 / 333$ \\
\hline Goodness-of-fit on $\mathrm{F}^{2}$ & 1.046 \\
\hline Final $R$ indexes $[\mid>=2 \sigma(I)]$ & $\mathrm{R}_{1}=0.0823, \mathrm{wR}_{2}=0.2221$ \\
\hline Final $R$ indexes [all data] & $\mathrm{R}_{1}=0.0866, \mathrm{wR}_{2}=0.2282$ \\
\hline Largest diff. peak/hole / e $\AA^{-3}$ & $0.93 /-0.47$ \\
\hline
\end{tabular}


Table S4. Crystal data and structure refinement for 1i (CCDC 1990406; triflate anion and H atoms on Comitted for clarity).

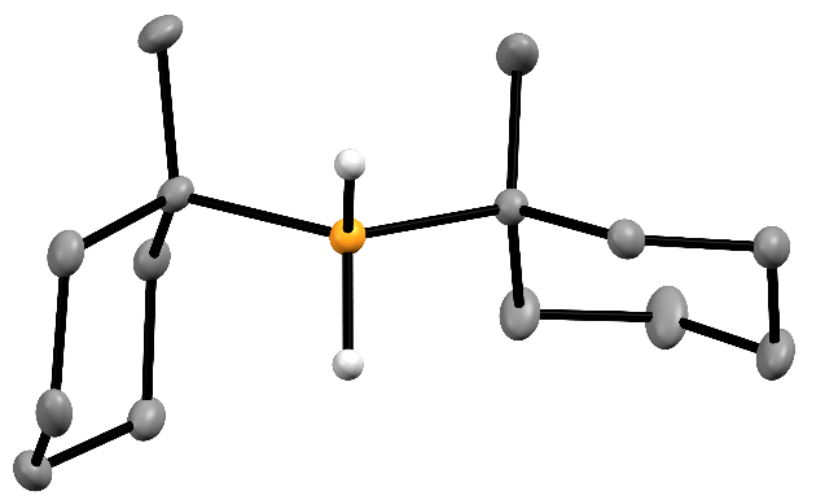

\begin{tabular}{|c|c|}
\hline Empirical formula & $\mathrm{C}_{15} \mathrm{H}_{28} \mathrm{~F}_{3} \mathrm{O}_{3} \mathrm{PS}$ \\
\hline Formula weight & 376.40 \\
\hline Temperature/K & $120(2)$ \\
\hline Crystal system & monoclinic \\
\hline Space group & $\mathrm{P} 22_{1} / \mathrm{n}$ \\
\hline $\mathrm{a} / \AA ̊$ & $9.5000(2)$ \\
\hline $\mathrm{b} / \AA ̊$ & $10.6274(2)$ \\
\hline$c / \AA$ & $18.6029(4)$ \\
\hline$\alpha /^{\circ}$ & 90 \\
\hline$\beta /^{\circ}$ & $100.595(2)$ \\
\hline$\gamma /{ }^{\circ}$ & 90 \\
\hline Volume $/ \AA^{3}$ & $1846.13(7)$ \\
\hline Z & 4 \\
\hline$\rho_{\text {calc }} \mathrm{g} / \mathrm{cm}^{3}$ & 1.354 \\
\hline$\mu / \mathrm{mm}^{-1}$ & 2.735 \\
\hline$F(000)$ & 800 \\
\hline Crystal size $/ \mathrm{mm}^{3}$ & $0.210 \times 0.183 \times 0.118$ \\
\hline Radiation & $\operatorname{CuK} \alpha(\lambda=1.54184)$ \\
\hline $2 \Theta$ range for data collection ${ }^{\circ}$ & 9.6260 to 147.5540 \\
\hline Index ranges & $-11 \leq h \leq 7,-12 \leq k \leq 11,-22 \leq \mathrm{I} \leq 23$ \\
\hline Reflections collected & 6454 \\
\hline Independent reflections & $6454\left[R_{\text {int }}=0.0389, R_{\text {sigma }}=0.0461\right]$ \\
\hline Data/restraints/parameters & $3553 / 1 / 216$ \\
\hline Goodness-of-fit on $\mathrm{F}^{2}$ & 1.040 \\
\hline Final $R$ indexes $[I>=2 \sigma(I)]$ & $\mathrm{R}_{1}=0.0533 ; \mathrm{wR}_{2}=0.1293$ \\
\hline Final $R$ indexes [all data] & $\mathrm{R}_{1}=0.0574 ; \mathrm{wR}_{2}=0.1341$ \\
\hline Largest diff. peak/hole / e $\AA^{-3}$ & $1.044 /-0.673$ \\
\hline
\end{tabular}


Table S5. Crystal data and structure refinement for $\mathbf{1 k}$ (CCDC 1990407; triflate anion and H atoms on Comitted for clarity).

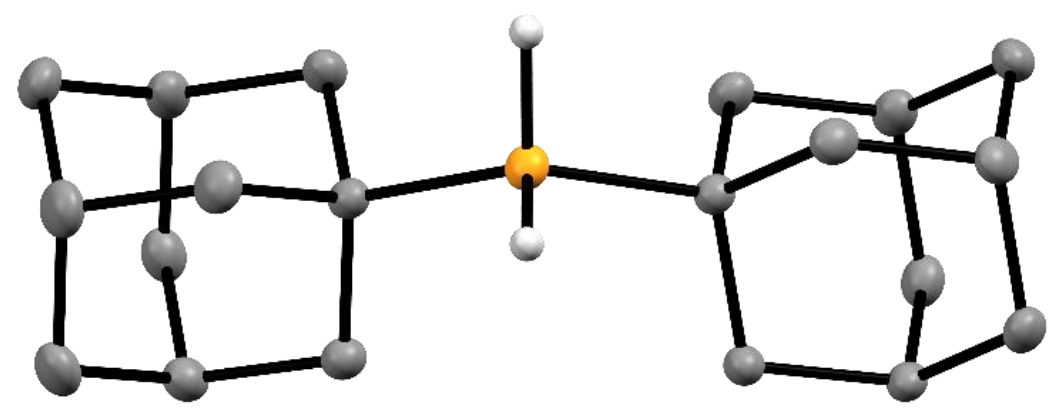

\begin{tabular}{|c|c|}
\hline Empirical formula & $\mathrm{C}_{21} \mathrm{H}_{32} \mathrm{~F}_{3} \mathrm{O}_{3} \mathrm{PS}$ \\
\hline Formula weight & 452.49 \\
\hline Temperature/K & $120(2)$ \\
\hline Crystal system & monoclinic \\
\hline Space group & $\mathrm{P} 22_{1} / \mathrm{c}$ \\
\hline $\mathrm{a} / \AA ̊ \AA$ & $11.1508(2)$ \\
\hline $\mathrm{b} / \AA ̊ \AA$ & $18.5177(4)$ \\
\hline$c / \AA ̊$ & $21.1099(5)$ \\
\hline$\alpha /^{\circ}$ & 90 \\
\hline$\beta /^{\circ}$ & $100.488(2)$ \\
\hline$\gamma /{ }^{\circ}$ & 90 \\
\hline Volume $/ \AA^{3}$ & $4286.10(16)$ \\
\hline Z & 8 \\
\hline$\rho_{\text {calc }} \mathrm{g} / \mathrm{cm}^{3}$ & 1.402 \\
\hline$\mu / \mathrm{mm}^{-1}$ & 2.457 \\
\hline$F(000)$ & 1920 \\
\hline Crystal size $/ \mathrm{mm}^{3}$ & $0.158 \times 0.077 \times 0.043$ \\
\hline Radiation & $\operatorname{CuK} \alpha(\lambda=1.54184)$ \\
\hline $2 \Theta$ range for data collection $/^{\circ}$ & 6.396 to 147.522 \\
\hline Index ranges & $-12 \leq h \leq 13,-23 \leq k \leq 20,-26 \leq \mathrm{I} \leq 17$ \\
\hline Reflections collected & 28854 \\
\hline Independent reflections & $8144\left[R_{\text {int }}=0.0676, R_{\text {sigma }}=0.0408\right]$ \\
\hline Data/restraints/parameters & $8144 / 6 / 539$ \\
\hline Goodness-of-fit on $F^{2}$ & 1.039 \\
\hline Final $R$ indexes $[\mid>=2 \sigma(I)]$ & $R_{1}=0.0642 ; w R_{2}=0.1644$ \\
\hline Final $R$ indexes [all data] & $R_{1}=0.0746 ; w R_{2}=0.1749$ \\
\hline Largest diff. peak/hole / e $\AA^{-3}$ & $1.836 /-0.706$ \\
\hline
\end{tabular}


Table S6. Crystal data and structure refinement for $1 \mathrm{~m}$ (CCDC 1990408; triflate anion and $\mathrm{H}$ atoms on Comitted for clarity).

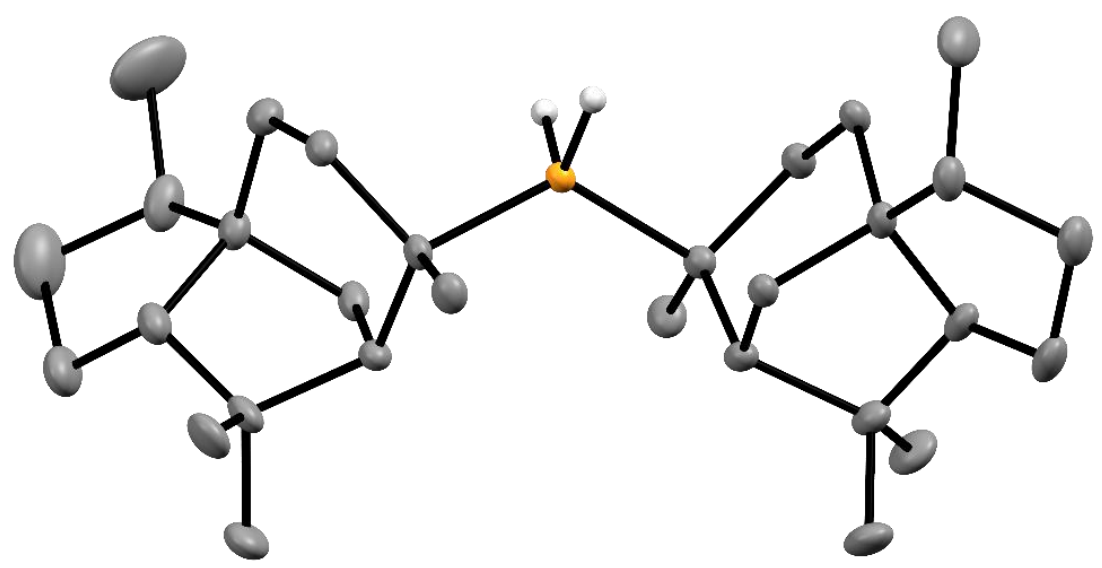

\begin{tabular}{|c|c|}
\hline Empirical formula & $\mathrm{C}_{31} \mathrm{H}_{52} \mathrm{~F}_{3} \mathrm{O}_{3} \mathrm{PS}$ \\
\hline Formula weight & 592.75 \\
\hline Temperature/K & $120(2)$ \\
\hline Crystal system & orthorhombic \\
\hline Space group & $\mathrm{P} 2{ }_{1}{ }_{1}{ }_{2}{ }_{1}$ \\
\hline $\mathrm{a} / \AA ̊ \AA$ & $7.3620(2)$ \\
\hline $\mathrm{b} / \AA ̊$ & $16.8285(3)$ \\
\hline$c / \AA$ & $25.1572(5)$ \\
\hline$\alpha /^{\circ}$ & 90 \\
\hline$\beta /^{\circ}$ & 90 \\
\hline$\gamma /{ }^{\circ}$ & 90 \\
\hline Volume $/ \AA^{3}$ & $3116.76(12)$ \\
\hline Z & 4 \\
\hline$\rho_{\text {calc }} \mathrm{g} / \mathrm{cm}^{3}$ & 1.263 \\
\hline$\mu / \mathrm{mm}^{-1}$ & 1.807 \\
\hline$F(000)$ & 1280.0 \\
\hline Crystal size $/ \mathrm{mm}^{3}$ & $0.45 \times 0.098 \times 0.033$ \\
\hline Radiation & CuK $\alpha(\lambda=1.54184)$ \\
\hline $2 \Theta$ range for data collection ${ }^{\circ}$ & 7.028 to 145.772 \\
\hline Index ranges & $-8 \leq h \leq 7,-20 \leq k \leq 20,-31 \leq 1 \leq 29$ \\
\hline Reflections collected & 18408 \\
\hline Independent reflections & $6075\left[R_{\text {int }}=0.0897, R_{\text {sigma }}=0.0594\right]$ \\
\hline Data/restraints/parameters & $6075 / 1 / 366$ \\
\hline Goodness-of-fit on $\mathrm{F}^{2}$ & 1.041 \\
\hline Final $R$ indexes $[\mid>=2 \sigma(I)]$ & $R_{1}=0.0666, w R_{2}=0.1796$ \\
\hline Final $\mathrm{R}$ indexes [all data] & $R_{1}=0.0692, w R_{2}=0.1837$ \\
\hline Largest diff. peak/hole / e $\AA^{-3}$ & $0.67 /-0.40$ \\
\hline Flack parameter & $-0.013(13)$ \\
\hline
\end{tabular}


Table S7. Crystal data and structure refinement for 1 ( (CCDC 1990409; triflate anion, $\mathrm{H}$ atoms on C and one molecule of $\mathrm{CH}_{2} \mathrm{Cl}_{2}$ omitted for clarity).

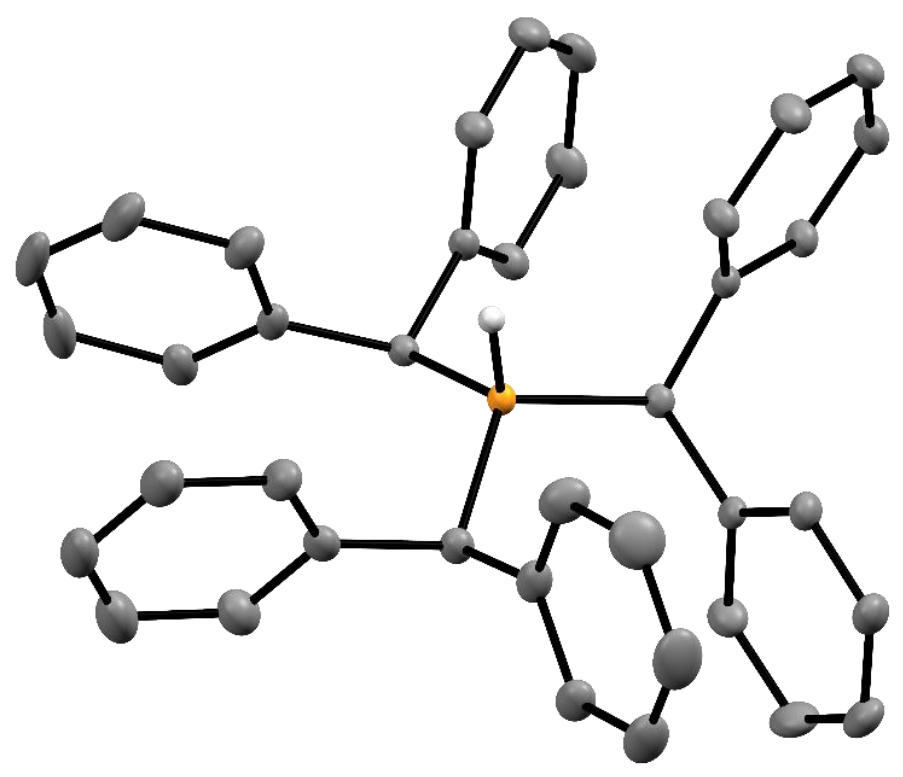

\begin{tabular}{|c|c|}
\hline Empirical formula & $\mathrm{C}_{40.9} \mathrm{H}_{35.8} \mathrm{Cl}_{1.8} \mathrm{~F}_{3} \mathrm{O}_{3} \mathrm{PS}$ \\
\hline Formula weight & 759.13 \\
\hline Temperature/K & $120(2)$ \\
\hline Crystal system & monoclinic \\
\hline Space group & $\mathrm{P} 2_{1} / \mathrm{c}$ \\
\hline$a / \AA ̊$ & $11.5515(2)$ \\
\hline $\mathrm{b} / \mathrm{A}$ & $18.5889(2)$ \\
\hline$c / \AA ̊$ & $17.7571(2)$ \\
\hline$\alpha /^{\circ}$ & 90 \\
\hline$\beta /^{\circ}$ & $99.9070(10)$ \\
\hline$\gamma /{ }^{\circ}$ & 90 \\
\hline Volume $/ \AA^{3}$ & $3756.12(9)$ \\
\hline Z & 4 \\
\hline$\rho_{\text {calc }} g / \mathrm{cm}^{3}$ & 1.342 \\
\hline$\mu / \mathrm{mm}^{-1}$ & 2.789 \\
\hline$F(000)$ & 1575.0 \\
\hline Crystal size $/ \mathrm{mm}^{3}$ & $0.574 \times 0.213 \times 0.052$ \\
\hline Radiation & $\operatorname{CuK} \alpha(\lambda=1.54184)$ \\
\hline \multicolumn{2}{|c|}{$2 \Theta$ range for data collection $/{ }^{\circ} 7.77$ to 147.506} \\
\hline Index ranges & $-14 \leq h \leq 14,-23 \leq k \leq 23,-16 \leq \mathrm{I} \leq 22$ \\
\hline Reflections collected & 61659 \\
\hline Independent reflections & $7546\left[R_{\text {int }}=0.0651, R_{\text {sigma }}=0.0278\right]$ \\
\hline Data/restraints/parameters & $7546 / 496 / 555$ \\
\hline Goodness-of-fit on $F^{2}$ & 1.026 \\
\hline Final $R$ indexes $[\mid>=2 \sigma(I)]$ & $\mathrm{R}_{1}=0.0701, \mathrm{wR}_{2}=0.1794$ \\
\hline Final R indexes [all data] & $\mathrm{R}_{1}=0.0726, \mathrm{wR}_{2}=0.1818$ \\
\hline Largest diff. peak/hole / e $\AA^{-3}$ & $1.10 /-0.63$ \\
\hline
\end{tabular}


Table S8. Crystal data and structure refinement for $3 f$ (CCDC 1990410; $\mathrm{H}$ atoms omitted for clarity).

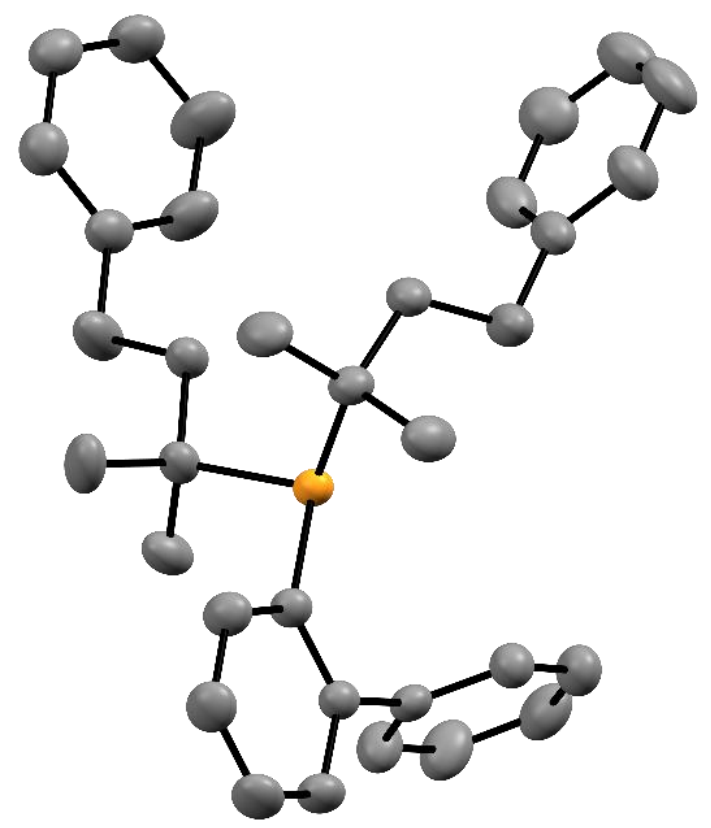

\begin{tabular}{|c|c|}
\hline Empirical formula & $\mathrm{C}_{34} \mathrm{H}_{39} \mathrm{P}$ \\
\hline Formula weight & 478.62 \\
\hline Temperature/K & $120(2)$ \\
\hline Crystal system & triclinic \\
\hline Space group & $\mathrm{P}-1$ \\
\hline $\mathrm{a} / \AA ̊$ & $10.1139(2)$ \\
\hline b/Å & $12.6246(3)$ \\
\hline$c / \AA ̊$ & $13.5249(3)$ \\
\hline$\alpha /^{\circ}$ & $111.882(2)$ \\
\hline$\beta /{ }^{\circ}$ & $109.918(2)$ \\
\hline$\gamma /{ }^{\circ}$ & $96.579(2)$ \\
\hline Volume $/ \AA^{3}$ & $1447.74(6)$ \\
\hline $\mathrm{Z}$ & 2 \\
\hline$\rho_{\text {calc }} \mathrm{g} / \mathrm{cm}^{3}$ & 1.098 \\
\hline$\mu / \mathrm{mm}^{-1}$ & 0.962 \\
\hline$F(000)$ & 516.0 \\
\hline Crystal size $/ \mathrm{mm}^{3}$ & $0.158 \times 0.149 \times 0.071$ \\
\hline Radiation & CuK $\alpha(\lambda=1.54184)$ \\
\hline \multicolumn{2}{|c|}{$2 \Theta$ range for data collection $/{ }^{\circ} 7.746$ to 144.51} \\
\hline Index ranges & $-12 \leq h \leq 12,-15 \leq k \leq 15,-16 \leq \mathrm{I} \leq 16$ \\
\hline Reflections collected & 23255 \\
\hline Independent reflections & $5573\left[R_{\text {int }}=0.0280, R_{\text {sigma }}=0.0188\right]$ \\
\hline Data/restraints/parameters & $5573 / 401 / 375$ \\
\hline Goodness-of-fit on $\mathrm{F}^{2}$ & 1.043 \\
\hline Final $R$ indexes $[1>=2 \sigma(I)]$ & $\mathrm{R}_{1}=0.0381, \mathrm{wR}_{2}=0.1005$ \\
\hline Final R indexes [all data] & $\mathrm{R}_{1}=0.0435, w \mathrm{R}_{2}=0.1057$ \\
\hline Largest diff. peak/hole / e $\AA^{-3}$ & $0.21 /-0.16$ \\
\hline
\end{tabular}


Table S9. Crystal data and structure refinement for 5a (CCDC 1990411; $\mathrm{H}$ atoms and one molecule of cyclohexane omitted for clarity).

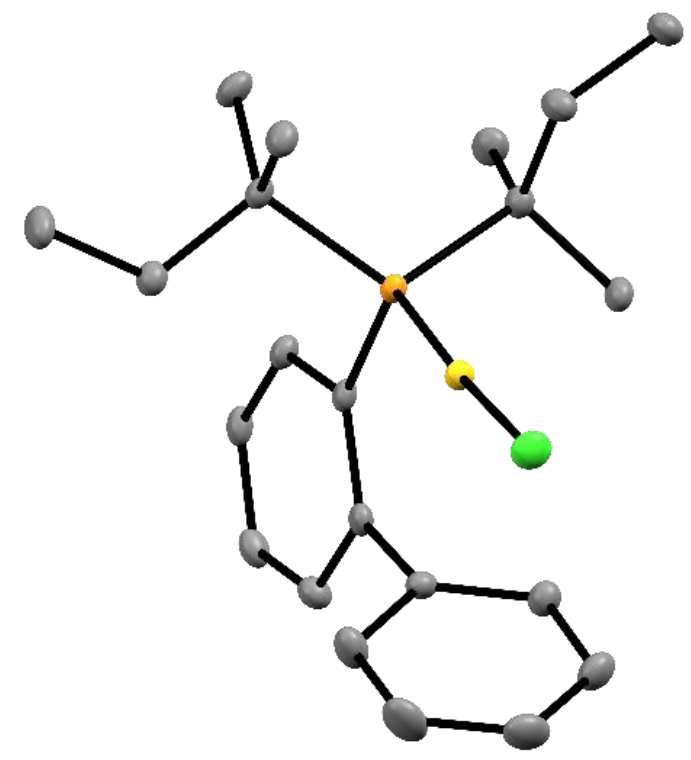

\begin{tabular}{|c|c|}
\hline Empirical formula & $\mathrm{C}_{47} \mathrm{H}_{68} \mathrm{Au}_{2} \mathrm{Cl}_{2} \mathrm{P}_{2}$ \\
\hline Formula weight & 1159.78 \\
\hline Temperature/K & $120(2)$ \\
\hline Crystal system & triclinic \\
\hline Space group & $\mathrm{P}-1$ \\
\hline $\mathrm{a} / \AA ̊ \AA$ & $10.0872(3)$ \\
\hline $\mathrm{b} / \AA ̊ \AA$ & $14.0744(6)$ \\
\hline$c / \AA ̊$ & $16.7016(5)$ \\
\hline$\alpha /^{\circ}$ & $78.850(3)$ \\
\hline$\beta /^{\circ}$ & $81.860(2)$ \\
\hline$\gamma /{ }^{\circ}$ & $78.924(3)$ \\
\hline Volume $/ \AA^{3}$ & 2269.59(14) \\
\hline Z & 2 \\
\hline$\rho_{\text {calc }} / \mathrm{cm}^{3}$ & 1.697 \\
\hline$\mu / \mathrm{mm}^{-1}$ & 6.676 \\
\hline$F(000)$ & 1144.0 \\
\hline Crystal size $/ \mathrm{mm}^{3}$ & $0.178 \times 0.143 \times 0.036$ \\
\hline Radiation & $\operatorname{MoK} \alpha(\lambda=0.71073)$ \\
\hline \multicolumn{2}{|c|}{$2 \Theta$ range for data collection $/{ }^{\circ} 5.988$ to 70.126} \\
\hline Index ranges & $-16 \leq h \leq 15,-22 \leq k \leq 22,-26 \leq 1 \leq 26$ \\
\hline Reflections collected & 64649 \\
\hline Independent reflections & $18791\left[R_{\text {int }}=0.0359, R_{\text {sigma }}=0.0395\right]$ \\
\hline Data/restraints/parameters & $18791 / 656 / 631$ \\
\hline Goodness-of-fit on $\mathrm{F}^{2}$ & 1.034 \\
\hline Final $R$ indexes $[\mid>=2 \sigma(I)]$ & $\mathrm{R}_{1}=0.0285, \mathrm{wR}_{2}=0.0483$ \\
\hline Final $R$ indexes [all data] & $R_{1}=0.0462, w R_{2}=0.0539$ \\
\hline Largest diff. peak/hole / e $\AA^{-3}$ & $1.23 /-1.66$ \\
\hline
\end{tabular}


Table S10. Crystal data and structure refinement for 5b (CCDC 1990412; $\mathrm{H}$ atoms omitted for clarity).

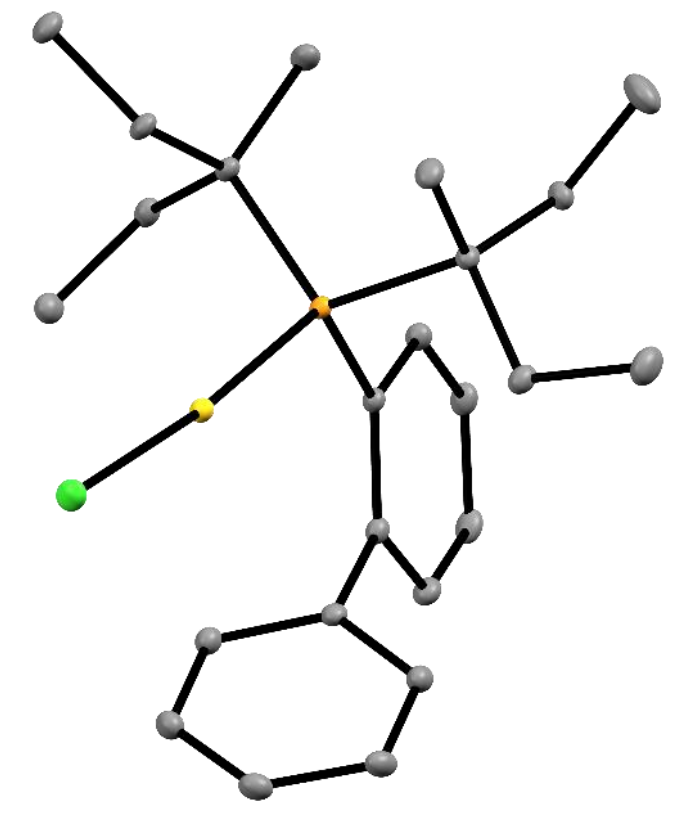

\begin{tabular}{|c|c|}
\hline Empirical formula & $\mathrm{C}_{24} \mathrm{H}_{35} \mathrm{AuClP}$ \\
\hline Formula weight & 586.90 \\
\hline Temperature/K & $120(2)$ \\
\hline Crystal system & monoclinic \\
\hline Space group & $\mathrm{P} 2{ }_{1} / \mathrm{n}$ \\
\hline$a / \AA ̊$ & $10.0134(2)$ \\
\hline $\mathrm{b} / \AA ̊$ & $14.7285(3)$ \\
\hline$c / \AA ̊$ & $15.0955(3)$ \\
\hline$\alpha /^{\circ}$ & 90 \\
\hline$\beta /^{\circ}$ & $92.029(2)$ \\
\hline$\gamma /{ }^{\circ}$ & 90 \\
\hline Volume $/ \AA^{3}$ & $2224.92(8)$ \\
\hline Z & 4 \\
\hline$\rho_{\text {calc }} g / \mathrm{cm}^{3}$ & 1.752 \\
\hline$\mu / \mathrm{mm}^{-1}$ & 6.811 \\
\hline$F(000)$ & 1160.0 \\
\hline Crystal size $/ \mathrm{mm}^{3}$ & $0.261 \times 0.23 \times 0.095$ \\
\hline Radiation & $\operatorname{MoK} \alpha(\lambda=0.71073)$ \\
\hline \multicolumn{2}{|c|}{$2 \Theta$ range for data collection $/{ }^{\circ} 6.068$ to 75.594} \\
\hline Index ranges & $-17 \leq h \leq 16,-24 \leq k \leq 25,-25 \leq \mathrm{I} \leq 25$ \\
\hline Reflections collected & 44846 \\
\hline Independent reflections & $11484\left[R_{\text {int }}=0.0414, R_{\text {sigma }}=0.0390\right]$ \\
\hline Data/restraints/parameters & $11484 / 0 / 251$ \\
\hline Goodness-of-fit on $F^{2}$ & 1.054 \\
\hline Final $R$ indexes $[\mid>=2 \sigma(I)]$ & $R_{1}=0.0249, w R_{2}=0.0467$ \\
\hline Final $R$ indexes [all data] & $\mathrm{R}_{1}=0.0355, \mathrm{wR}_{2}=0.0506$ \\
\hline Largest diff. peak/hole / e $\AA^{-3}$ & $1.73 /-1.56$ \\
\hline
\end{tabular}


Table S11. Crystal data and structure refinement for 5c (CCDC 1990413; $\mathrm{H}$ atoms omitted for clarity).

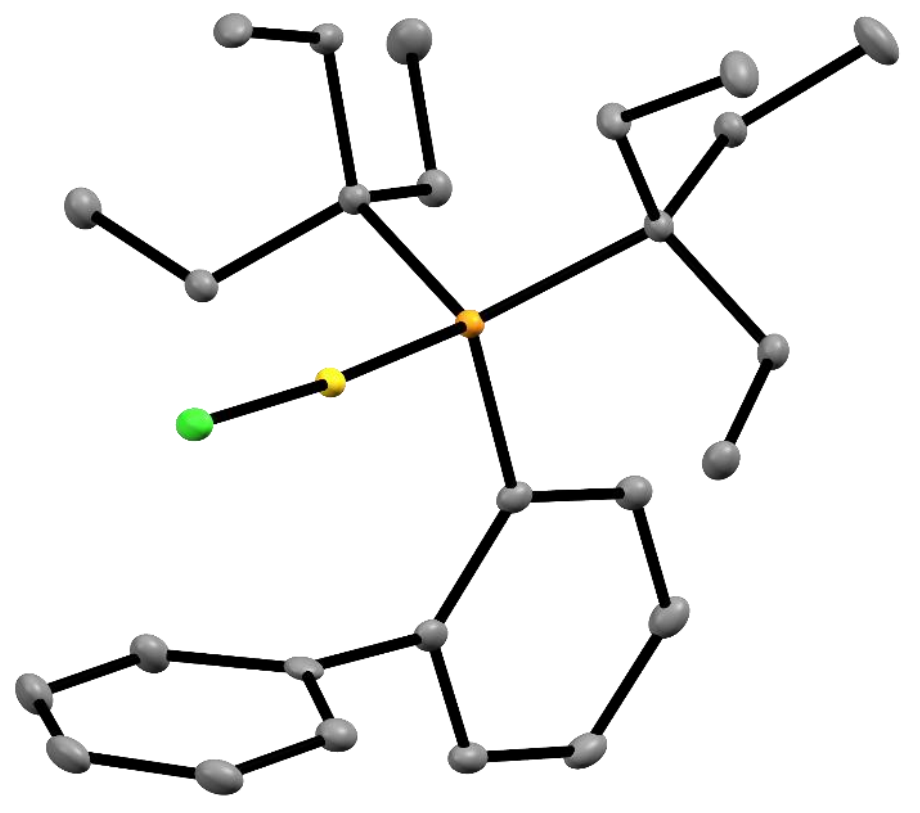

\begin{tabular}{|c|c|}
\hline Empirical formula & $\mathrm{C}_{26} \mathrm{H}_{39} \mathrm{AuClP}$ \\
\hline Formula weight & 614.96 \\
\hline Temperature/K & $120(2)$ \\
\hline Crystal system & monoclinic \\
\hline Space group & $\mathrm{P} 2{ }_{1} / \mathrm{n}$ \\
\hline$a / \AA ̊$ & $10.0412(4)$ \\
\hline $\mathrm{b} / \AA ̊$ & $15.8623(6)$ \\
\hline$c / \AA ̊$ & $15.2487(5)$ \\
\hline$\alpha /^{\circ}$ & 90 \\
\hline$\beta /^{\circ}$ & $91.963(4)$ \\
\hline$\gamma /{ }^{\circ}$ & 90 \\
\hline Volume $/ \AA^{3}$ & $2427.33(16)$ \\
\hline Z & 4 \\
\hline$\rho_{\text {calc }} \mathrm{g} / \mathrm{cm}^{3}$ & 1.683 \\
\hline$\mu / \mathrm{mm}^{-1}$ & 6.247 \\
\hline$F(000)$ & 1224.0 \\
\hline Crystal size $/ \mathrm{mm}^{3}$ & $0.099 \times 0.089 \times 0.052$ \\
\hline Radiation & $\operatorname{MoK} \alpha(\lambda=0.71073)$ \\
\hline \multicolumn{2}{|c|}{$2 \Theta$ range for data collection $/{ }^{\circ} 5.79$ to 57.374} \\
\hline Index ranges & $-13 \leq h \leq 11,-20 \leq k \leq 20,-18 \leq \mathrm{I} \leq 20$ \\
\hline Reflections collected & 24176 \\
\hline Independent reflections & $5556\left[R_{\text {int }}=0.0264, R_{\text {sigma }}=0.0232\right]$ \\
\hline Data/restraints/parameters & $5556 / 0 / 268$ \\
\hline Goodness-of-fit on $\mathrm{F}^{2}$ & 1.045 \\
\hline Final $R$ indexes $[\mid>=2 \sigma(I)]$ & $\mathrm{R}_{1}=0.0185, \mathrm{wR}_{2}=0.0387$ \\
\hline Final R indexes [all data] & $R_{1}=0.0223, w R_{2}=0.0399$ \\
\hline Largest diff. peak/hole / e $\AA^{-3}$ & $0.70 /-0.72$ \\
\hline
\end{tabular}


Table S12. Crystal data and structure refinement for 5d (CCDC 1990414; $\mathrm{H}$ atoms and two molecules of cyclohexane omitted for clarity).

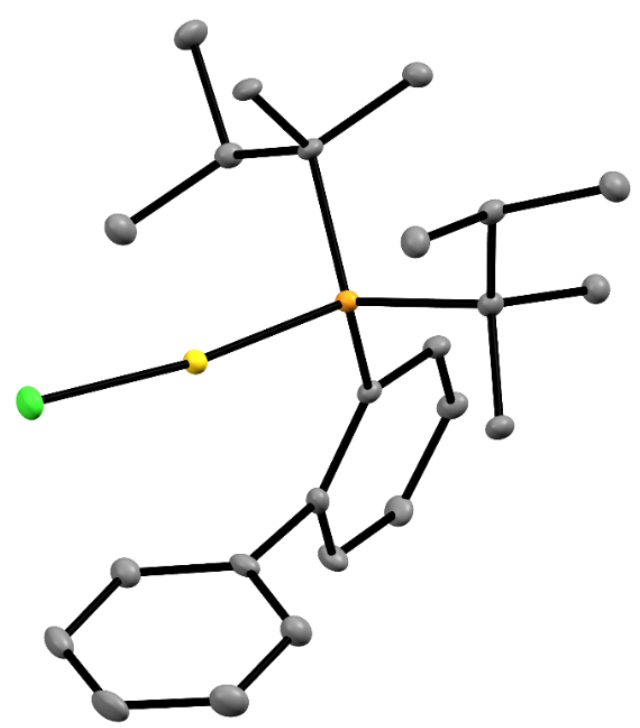

\begin{tabular}{|c|c|}
\hline Empirical formula & $\mathrm{C}_{30} \mathrm{H}_{47} \mathrm{AuClP}$ \\
\hline Formula weight & 671.06 \\
\hline Temperature/K & $120(2)$ \\
\hline Crystal system & monoclinic \\
\hline Space group & $\mathrm{P} 22_{1} / \mathrm{c}$ \\
\hline $\mathrm{a} / \AA ̊$ & $10.6600(3)$ \\
\hline$b / \AA ̊$ & $11.3550(3)$ \\
\hline$c / \AA$ & $23.7632(7)$ \\
\hline$\alpha /^{\circ}$ & 90 \\
\hline$\beta /^{\circ}$ & $100.803(3)$ \\
\hline$\gamma /{ }^{\circ}$ & 90 \\
\hline Volume $/ \AA^{3}$ & $2825.42(14)$ \\
\hline $\mathrm{Z}$ & 4 \\
\hline$\rho_{\text {calc }} \mathrm{g} / \mathrm{cm}^{3}$ & 1.578 \\
\hline$\mu / \mathrm{mm}^{-1}$ & 5.374 \\
\hline$F(000)$ & 1352.0 \\
\hline Crystal size $/ \mathrm{mm}^{3}$ & $0.153 \times 0.05 \times 0.017$ \\
\hline Radiation & $\operatorname{MoK} \alpha(\lambda=0.71073)$ \\
\hline \multicolumn{2}{|c|}{$2 \Theta$ range for data collection/ ${ }^{\circ} 5.796$ to 65.982} \\
\hline Index ranges & $-16 \leq h \leq 15,-16 \leq k \leq 17,-34 \leq 1 \leq 35$ \\
\hline Reflections collected & 64955 \\
\hline Independent reflections & $9978\left[R_{\text {int }}=0.0651, R_{\text {sigma }}=0.0530\right]$ \\
\hline Data/restraints/parameters & $9978 / 102 / 325$ \\
\hline Goodness-of-fit on $F^{2}$ & 1.116 \\
\hline Final $R$ indexes $[\mid>=2 \sigma(I)]$ & $\mathrm{R}_{1}=0.0371, \mathrm{wR}_{2}=0.0566$ \\
\hline Final R indexes [all data] & $\mathrm{R}_{1}=0.0619, \mathrm{wR}_{2}=0.0626$ \\
\hline Largest diff. peak/hole / e $\AA^{-3}$ & $1.97 /-1.15$ \\
\hline
\end{tabular}


Table S13. Crystal data and structure refinement for 5h (CCDC 1990415; H atoms and one molecule of water omitted for clarity).

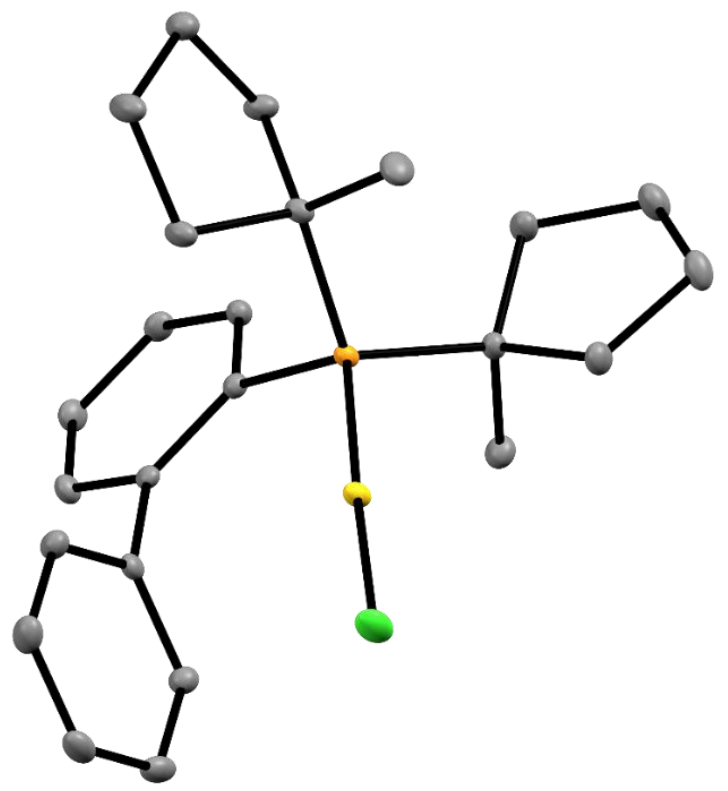

\begin{tabular}{|c|c|}
\hline Empirical formula & $\mathrm{C}_{24} \mathrm{H}_{31.22} \mathrm{AuClO}_{0.11} \mathrm{P}$ \\
\hline Formula weight & 584.85 \\
\hline Temperature/K & $120(2)$ \\
\hline Crystal system & monoclinic \\
\hline Space group & $\mathrm{P} 22_{1} / \mathrm{n}$ \\
\hline $\mathrm{a} / \AA ̊ \AA$ & $8.7107(2)$ \\
\hline $\mathrm{b} / \AA ̊$ & $9.9662(3)$ \\
\hline$c / \AA ̊$ & $25.5485(6)$ \\
\hline$\alpha /^{\circ}$ & 90 \\
\hline$\beta /^{\circ}$ & $96.659(2)$ \\
\hline$\gamma /{ }^{\circ}$ & 90 \\
\hline Volume $/ \AA^{3}$ & $2202.97(10)$ \\
\hline Z & 4 \\
\hline$\rho_{\text {calc }} / \mathrm{cm}^{3}$ & 1.763 \\
\hline$\mu / \mathrm{mm}^{-1}$ & 6.879 \\
\hline$F(000)$ & 1148.0 \\
\hline Crystal size $/ \mathrm{mm}^{3}$ & $0.113 \times 0.094 \times 0.051$ \\
\hline Radiation & $\operatorname{MoK} \alpha(\lambda=0.71073)$ \\
\hline \multicolumn{2}{|c|}{$2 \Theta$ range for data collection $/{ }^{\circ} 6.236$ to 57.416} \\
\hline Index ranges & $-11 \leq h \leq 10,-13 \leq k \leq 13,-33 \leq \mathrm{I} \leq 33$ \\
\hline Reflections collected & 55140 \\
\hline Independent reflections & $5357\left[R_{\text {int }}=0.0357, R_{\text {sigma }}=0.0192\right]$ \\
\hline Data/restraints/parameters & $5357 / 105 / 275$ \\
\hline Goodness-of-fit on $F^{2}$ & 1.033 \\
\hline Final $R$ indexes $[\mid>=2 \sigma(I)]$ & $R_{1}=0.0168, w R_{2}=0.0322$ \\
\hline Final $R$ indexes [all data] & $\mathrm{R}_{1}=0.0208, w \mathrm{R}_{2}=0.0336$ \\
\hline \multicolumn{2}{|c|}{ Largest diff. peak/hole / e $\AA^{-3} 0.79 /-0.73$} \\
\hline
\end{tabular}


Table S14. Crystal data and structure refinement for 5i (CCDC 1990416; $\mathrm{H}$ atoms omitted for clarity).

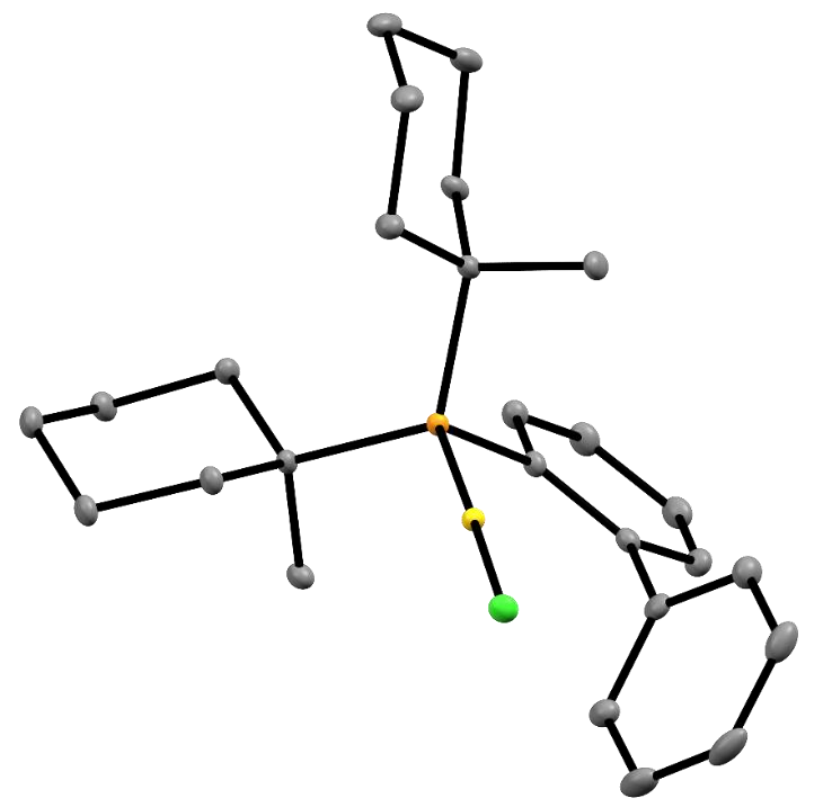

\begin{tabular}{|c|c|}
\hline Empirical formula & $\mathrm{C}_{26} \mathrm{H}_{35} \mathrm{AuClP}$ \\
\hline Formula weight & 610.92 \\
\hline Temperature/K & $120(2)$ \\
\hline Crystal system & monoclinic \\
\hline Space group & $\mathrm{P} 22_{1} / \mathrm{n}$ \\
\hline $\mathrm{a} / \AA ̊ \AA$ & $10.7821(3)$ \\
\hline $\mathrm{b} / \AA ̊ \AA$ & $20.3690(4)$ \\
\hline$c / \AA ̊$ & $11.1066(3)$ \\
\hline$\alpha /^{\circ}$ & 90 \\
\hline$\beta /{ }^{\circ}$ & $106.794(3)$ \\
\hline$\gamma /{ }^{\circ}$ & 90 \\
\hline Volume/ $/ \AA^{3}$ & $2335.20(11)$ \\
\hline Z & 4 \\
\hline$\rho_{\text {calc }} g / \mathrm{cm}^{3}$ & 1.738 \\
\hline$\mu / \mathrm{mm}^{-1}$ & 13.597 \\
\hline$F(000)$ & 1208 \\
\hline Crystal size $/ \mathrm{mm}^{3}$ & $0.23 \times 0.138 \times 0.05$ \\
\hline Radiation & $\operatorname{CuK} \alpha(\lambda=1.54184)$ \\
\hline $2 \Theta$ range for data collection $/^{\circ}$ & 9.382 to 148.956 \\
\hline Index ranges & $-12 \leq h \leq 13,-25 \leq k \leq 25,-13 \leq \mathrm{I} \leq 13$ \\
\hline Reflections collected & 19303 \\
\hline Independent reflections & $4705\left[R_{\text {int }}=0.0256, R_{\text {sigma }}=0.200\right]$ \\
\hline Data/restraints/parameters & $4705 / 0 / 265$ \\
\hline Goodness-of-fit on $F^{2}$ & 1.081 \\
\hline Final $R$ indexes $[I>=2 \sigma(I)]$ & $R_{1}=0.0177 ; w R_{2}=0.0428$ \\
\hline Final $R$ indexes [all data] & $\mathrm{R}_{1}=0.0188 ; w \mathrm{R}_{2}=0.0433$ \\
\hline Largest diff. peak/hole / e $\AA^{-3}$ & $0.728 /-0.966$ \\
\hline
\end{tabular}


Table S15. Crystal data and structure refinement for 5k (CCDC 1990417; $\mathrm{H}$ atoms omitted for clarity).

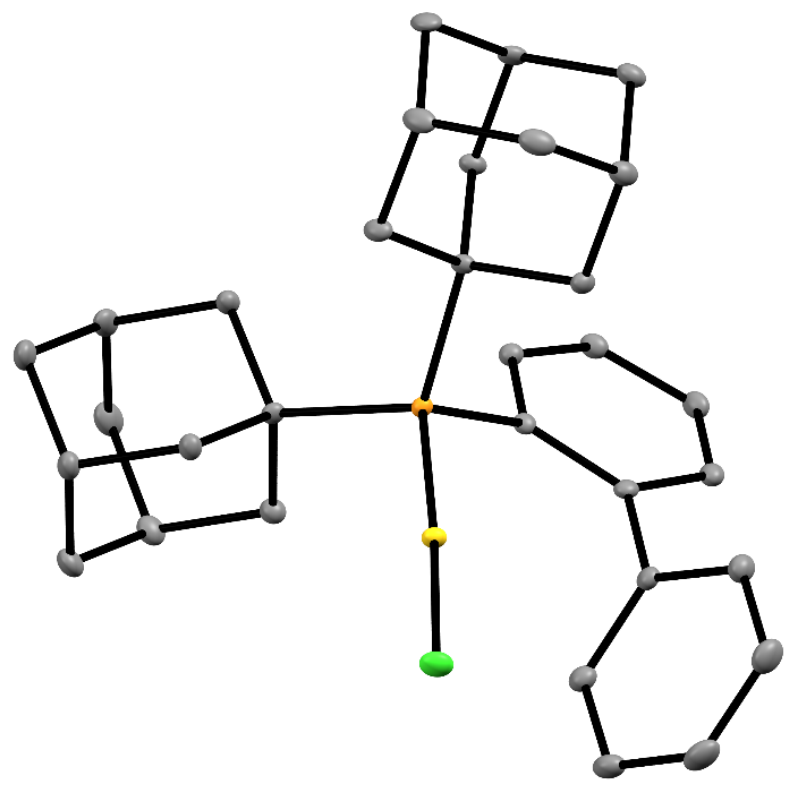

\begin{tabular}{|c|c|}
\hline Empirical formula & $\mathrm{C}_{32} \mathrm{H}_{39} \mathrm{AuClP}$ \\
\hline Formula weight & 687.02 \\
\hline Temperature/K & $120(2)$ \\
\hline Crystal system & monoclinic \\
\hline Space group & $\mathrm{P} 2{ }_{1} / \mathrm{c}$ \\
\hline $\mathrm{a} / \AA ̊$ & $11.9313(2)$ \\
\hline $\mathrm{b} / \AA ̊ \AA$ & $15.6446(2)$ \\
\hline$c / \AA ̊$ & $15.2881(2)$ \\
\hline$\alpha /^{\circ}$ & 90 \\
\hline$\beta /^{\circ}$ & $109.268(2)$ \\
\hline$\gamma /{ }^{\circ}$ & 90 \\
\hline Volume $/ \AA^{3}$ & $2693.83(7)$ \\
\hline Z & 4 \\
\hline$\rho_{\text {calc }} \mathrm{g} / \mathrm{cm}^{3}$ & 1.694 \\
\hline$\mu / \mathrm{mm}^{-1}$ & 5.640 \\
\hline$F(000)$ & 1368.0 \\
\hline Crystal size $/ \mathrm{mm}^{3}$ & $0.276 \times 0.09 \times 0.046$ \\
\hline Radiation & $\operatorname{MoK} \alpha(\lambda=0.71073)$ \\
\hline \multicolumn{2}{|c|}{$2 \Theta$ range for data collection $/{ }^{\circ} 5.61$ to 75.426} \\
\hline Index ranges & $-20 \leq h \leq 20,-26 \leq k \leq 26,-25 \leq 1 \leq 26$ \\
\hline Reflections collected & 51837 \\
\hline Independent reflections & $13779\left[R_{\text {int }}=0.0649, R_{\text {sigma }}=0.0720\right]$ \\
\hline Data/restraints/parameters & $13779 / 0 / 316$ \\
\hline Goodness-of-fit on $\mathrm{F}^{2}$ & 0.923 \\
\hline Final R indexes $[\mid>=2 \sigma(I)]$ & $R_{1}=0.0344, w R_{2}=0.0687$ \\
\hline Final R indexes [all data] & $\mathrm{R}_{1}=0.0587, \mathrm{wR}_{2}=0.0717$ \\
\hline Largest diff. peak/hole / e $\AA^{-3}$ & $3.37 /-1.89$ \\
\hline
\end{tabular}




\section{NMR Spectra}

3-Methyl-3-pentyl acetate - ${ }^{1} \mathrm{H}$ NMR $\left(500 \mathrm{MHz}^{\left.-\mathrm{CDCl}_{3}\right)}\right.$

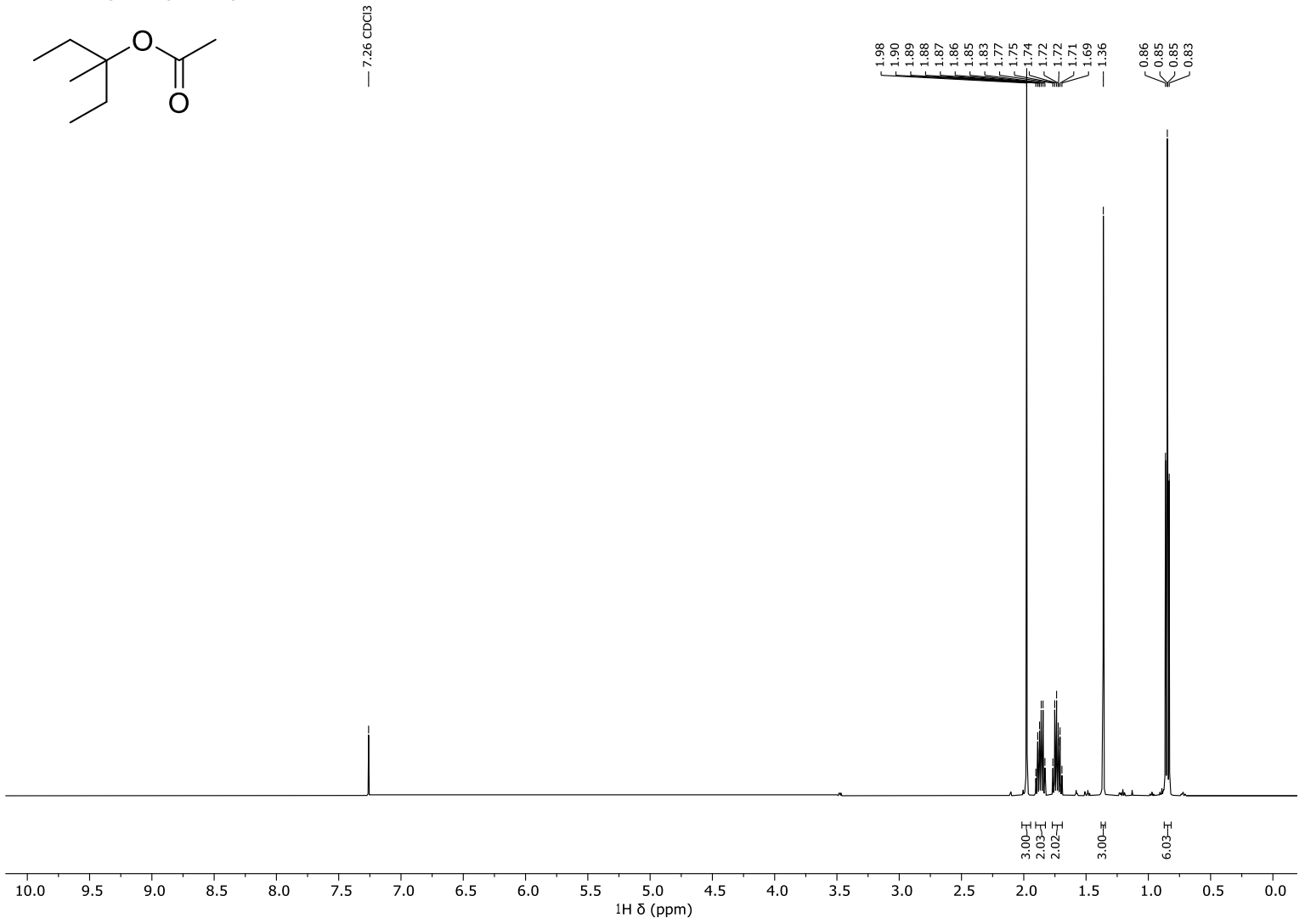

3-Methyl-3-pentyl acetate $-{ }^{13} \mathrm{C}\left\{{ }^{1} \mathrm{H}\right\} \mathrm{NMR}\left(126 \mathrm{MHz}, \mathrm{CDCl}_{3}\right)$<smiles>CCC(CC)(CC)OC(C)=O</smiles> 
3-Ethyl-3-pentyl acetate - ${ }^{1} \mathrm{H} \mathrm{NMR}\left(500 \mathrm{MHz}, \mathrm{CDCl}_{3}\right)$<smiles>CCC(CC)(CC)OC(C)=O</smiles>

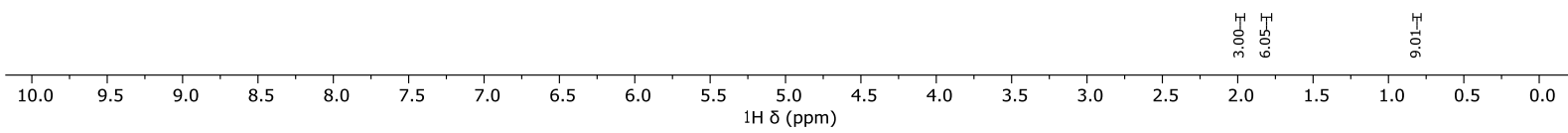

3-Ethyl-3-pentyl acetate $-{ }^{13} \mathrm{C}\left\{{ }^{1} \mathrm{H}\right\}$ NMR $\left(126 \mathrm{MHz}, \mathrm{CDCl}_{3}\right)$<smiles>CCC(CC)(CC)OC(C)=O</smiles>

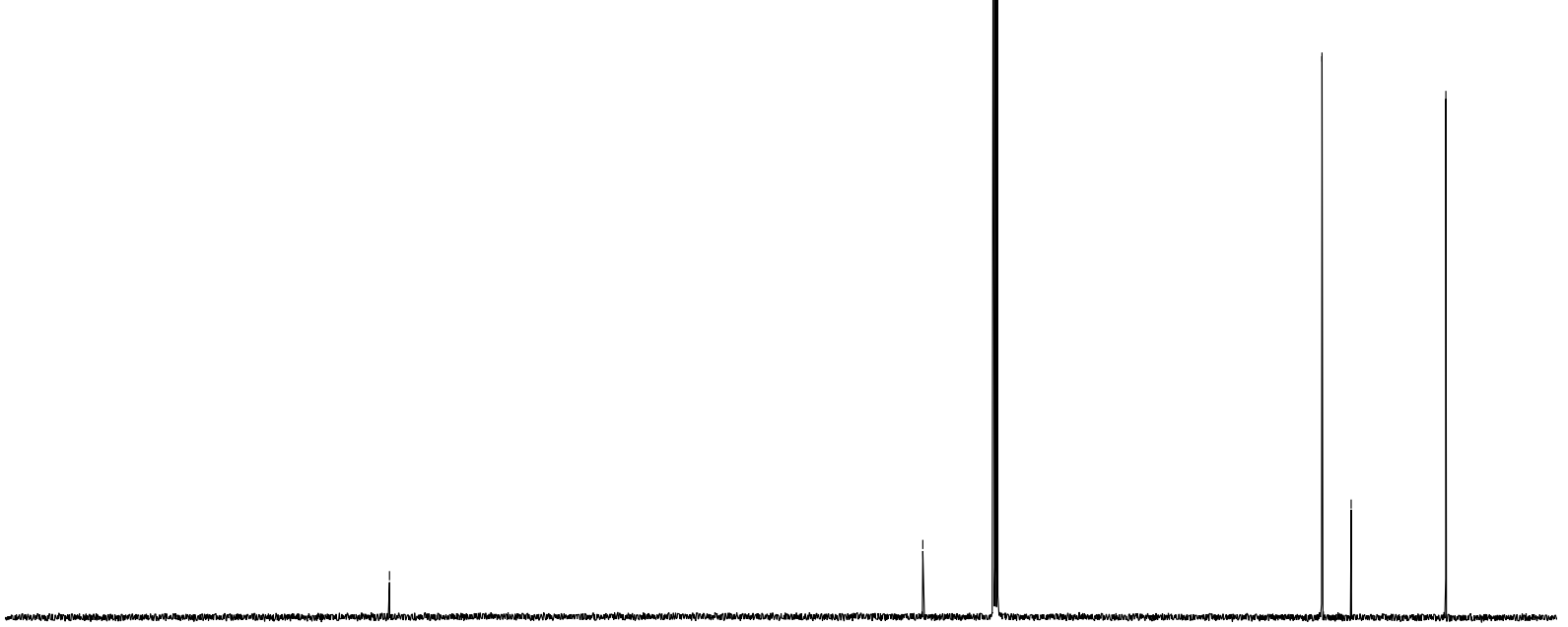

$\begin{array}{lllllllllll}220 & 210 & 200 & 190 & 180 & 170 & 160 & 150 & 140 & 130 & 120 \\ 13 \mathrm{C} \delta(\mathrm{ppm}) & 100\end{array}$ 
2,3-Dimethyl-2-butyl acetate - ${ }^{1} \mathrm{H} \mathrm{NMR}\left(500 \mathrm{MHz}, \mathrm{CDCl}_{3}\right)$<smiles>CC(=O)OC(C)(C)C(C)C</smiles>

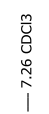

2,3-Dimethyl-2-butyl acetate $-{ }^{13} \mathrm{C}\left\{{ }^{1} \mathrm{H}\right\}$ NMR $\left(126 \mathrm{MHz}, \mathrm{CDCl}_{3}\right)$<smiles>CC(=O)OC(C)(C)C(C)C</smiles>

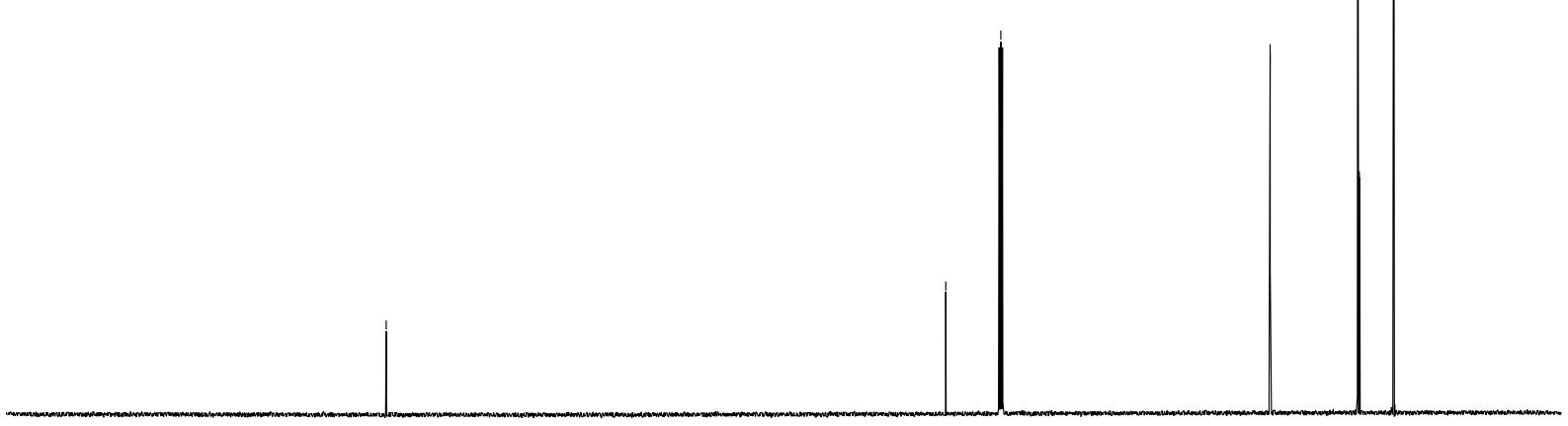




$$
=1
$$


2,3,3-Trimethyl-2-butyl acetate - ${ }^{1} \mathrm{H}$ NMR $\left(500 \mathrm{MHz}, \mathrm{CDCl}_{3}\right)$

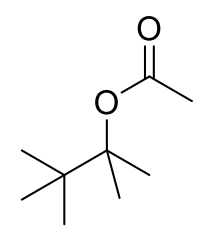

0
0
0
0

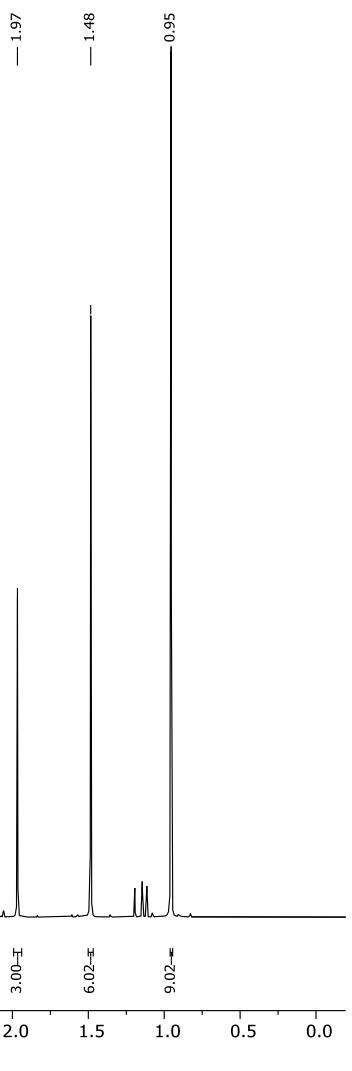

2,3,3-Trimethyl-2-butyl acetate $-{ }^{13} \mathrm{C}\left\{{ }^{1} \mathrm{H}\right\} \mathrm{NMR}\left(126 \mathrm{MHz}, \mathrm{CDCl}_{3}\right)$
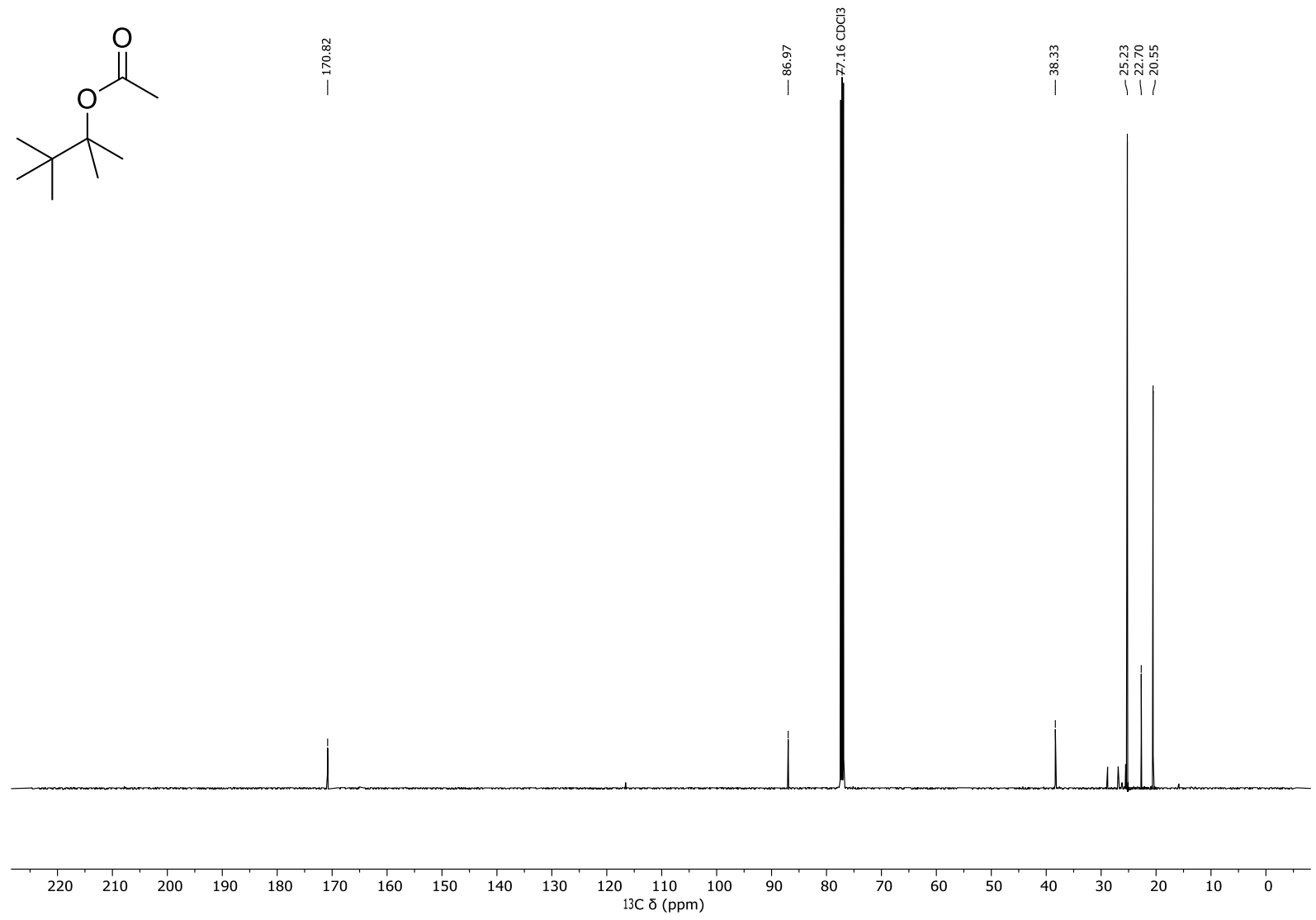

S99 
2-Methyl-4-(4-bromophenyl)-2-butanol - ${ }^{1} \mathrm{H}$ NMR (500 MHz, $\left.\mathrm{CDCl}_{3}\right)$

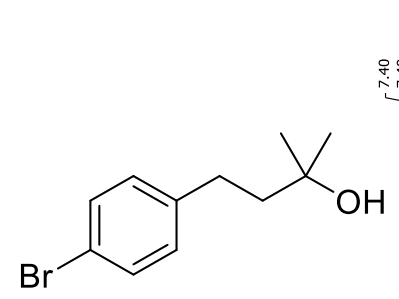

$\underbrace{1}$

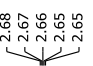

$\underbrace{2}$

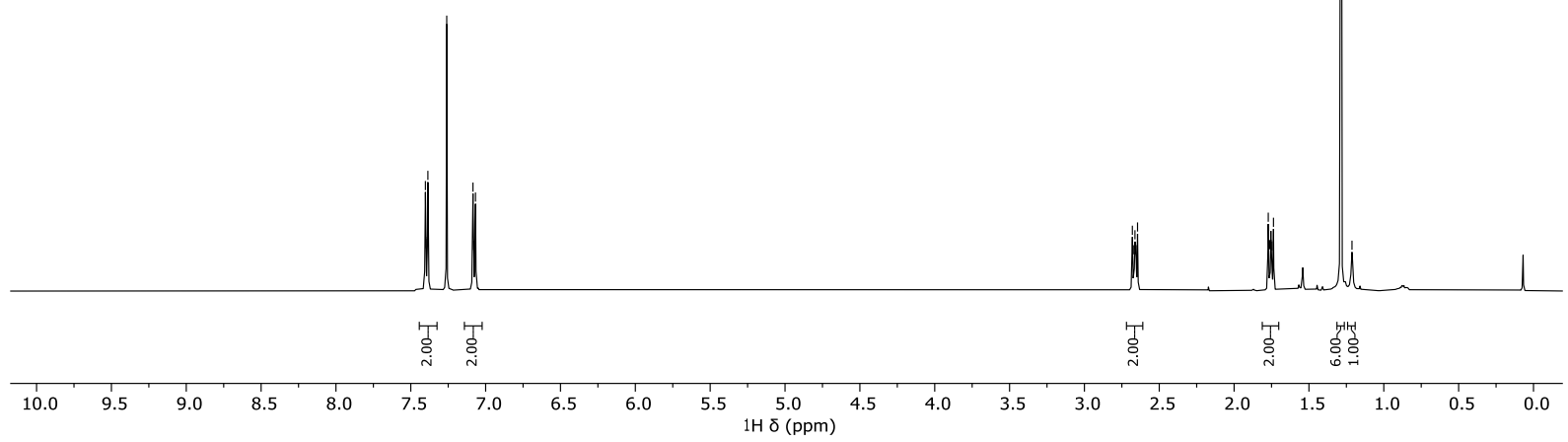

2-Methyl-4-(4-bromophenyl)-2-butanol - ${ }^{13} \mathrm{C}\left\{{ }^{1} \mathrm{H}\right\}$ NMR (126 MHz, $\left.\mathrm{CDCl}_{3}\right)$
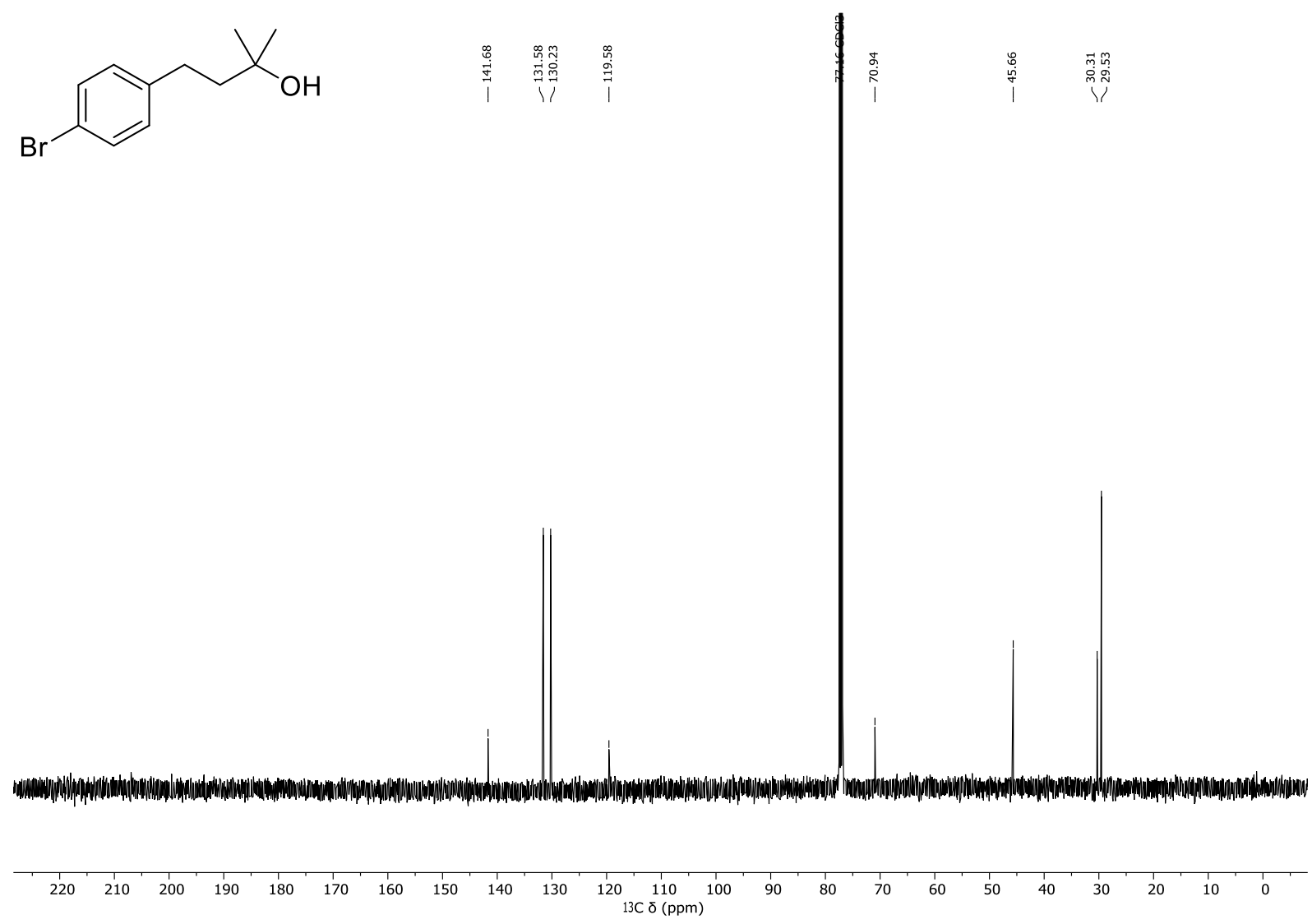

S100 
2-Methyl-4-(4-bromophenyl)-2-butyl acetate - ${ }^{1} \mathrm{H}$ NMR (500 MHz, $\left.\mathrm{CDCl}_{3}\right)$
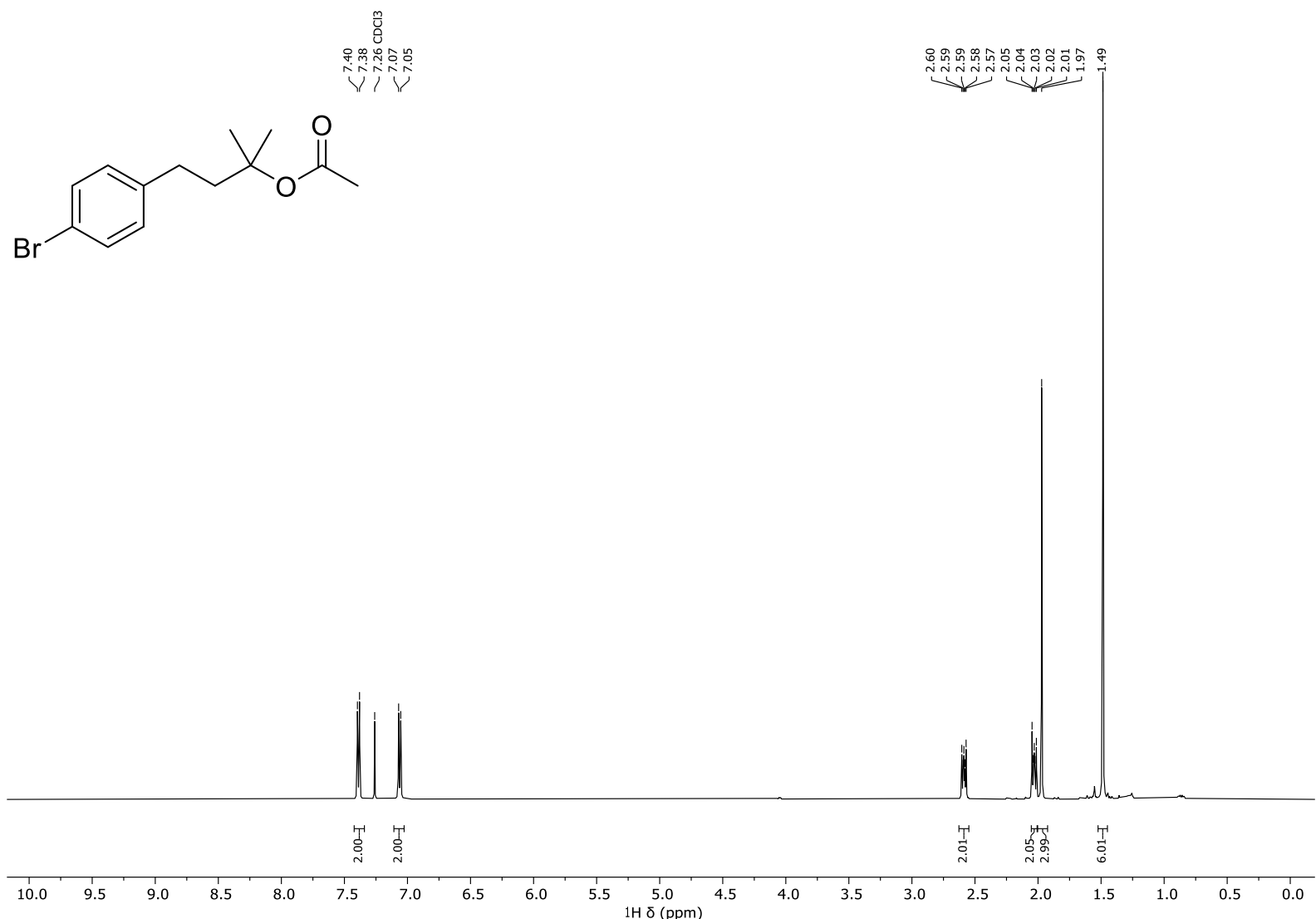

2-Methyl-4-(4-bromophenyl)-2-butyl acetate $-{ }^{13} \mathrm{C}\left\{{ }^{1} \mathrm{H}\right\} \mathrm{NMR}\left(126 \mathrm{MHz}, \mathrm{CDCl}_{3}\right)$

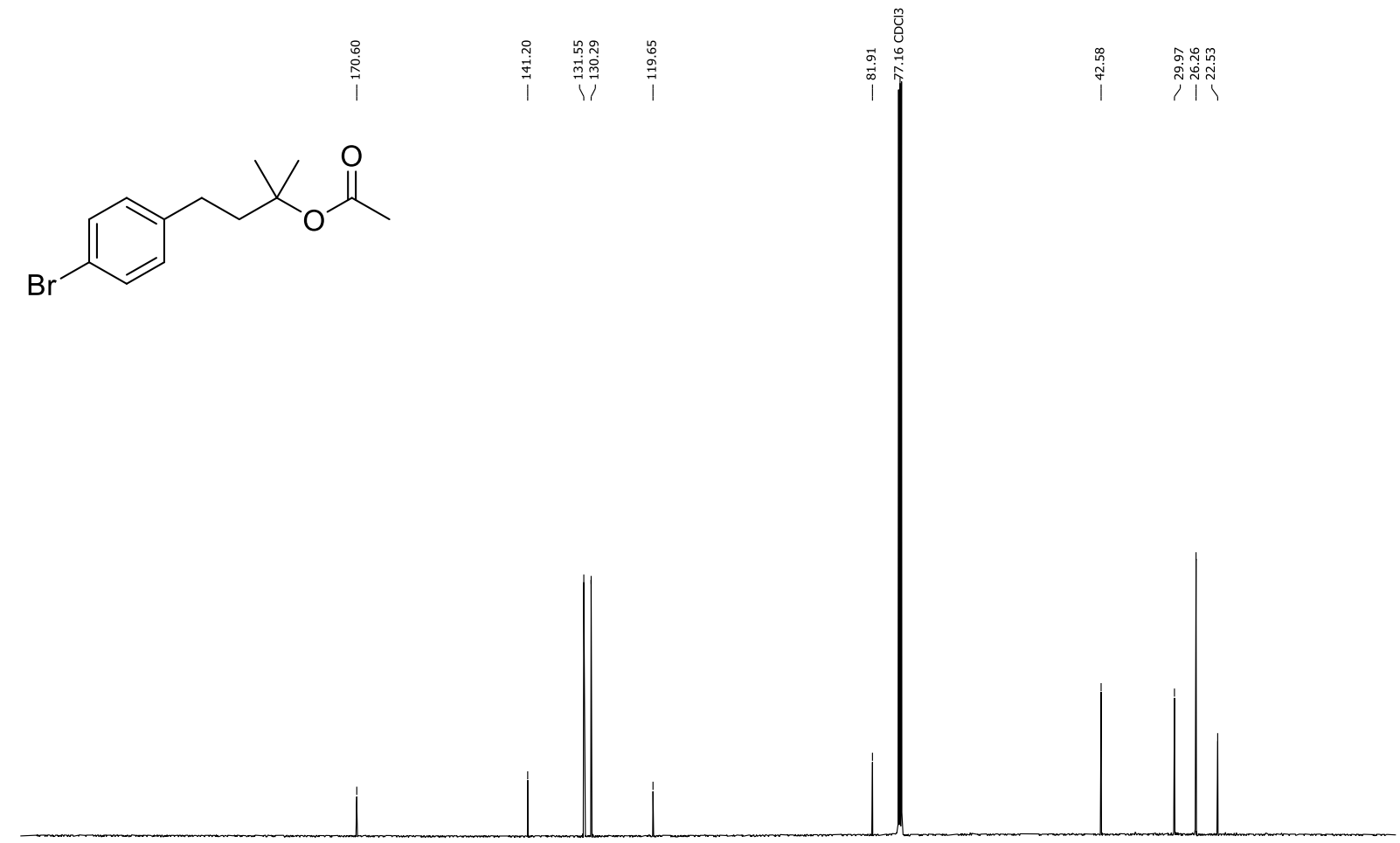

$\begin{array}{lllllllllllllllllllllllllllll}220 & 210 & 200 & 190 & 180 & 170 & 160 & 150 & 140 & 130 & 120 & 110 & 100 & 90 & 80 & 70 & 60 & 50 & 40 & 30 & 20 & 10 & 0\end{array}$ 
1-Methylcyclopentyl acetate $-{ }^{1} \mathrm{H} \mathrm{NMR}\left(500 \mathrm{MHz}, \mathrm{CDCl}_{3}\right)$

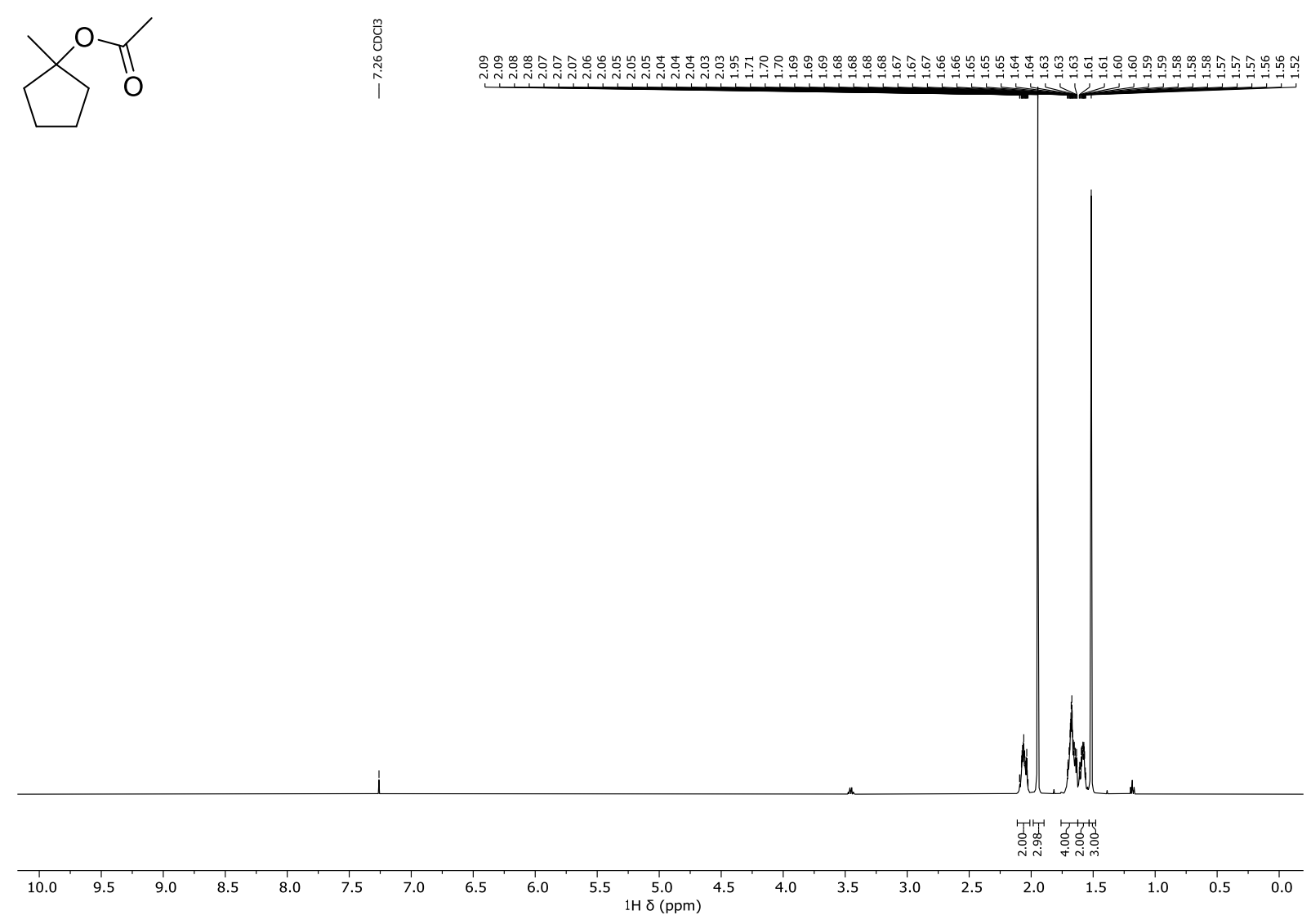

1-Methylcyclopentyl acetate $-{ }^{13} \mathrm{C}\left\{{ }^{1} \mathrm{H}\right\}$ NMR $\left(126 \mathrm{MHz}, \mathrm{CDCl}_{3}\right)$<smiles>CC(=O)OC1(C)CCCC1</smiles>

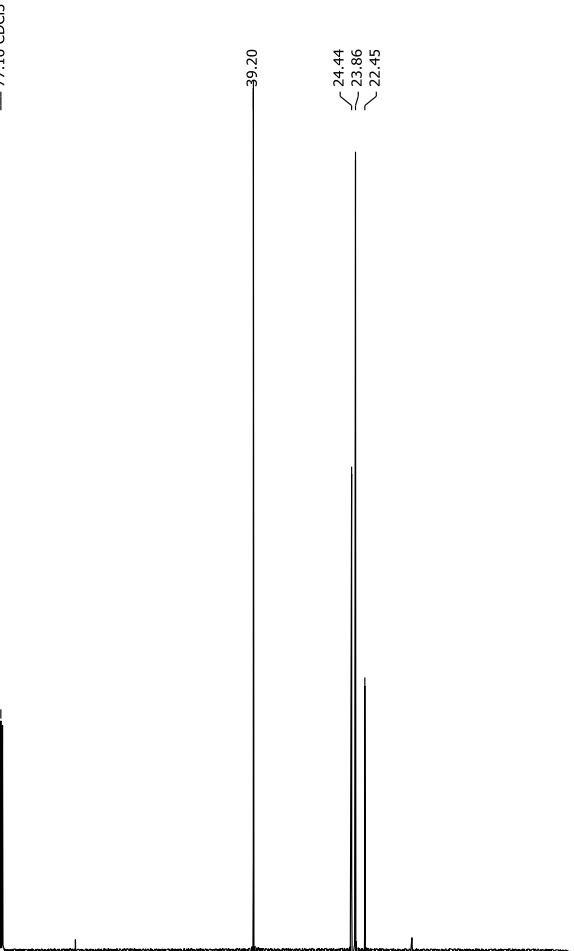

$\begin{array}{llllllllllllllllllllllllllll}220 & 210 & 200 & 190 & 180 & 170 & 160 & 150 & 140 & 130 & 120 & 110 & 100 & 90 & 80 & 70 & 60 & 50 & 40 & 30 & 20 & 10 & 0\end{array}$ 
1-Methylcyclohexyl acetate - ${ }^{1} \mathrm{H}$ NMR $\left(400 \mathrm{MHz}, \mathrm{CDCl}_{3}\right)$

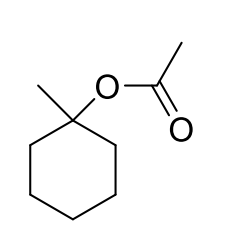

岩

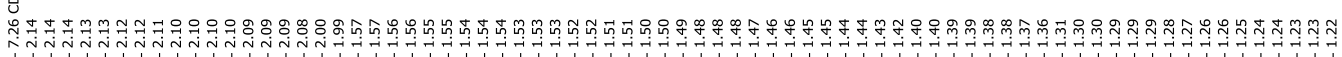

管
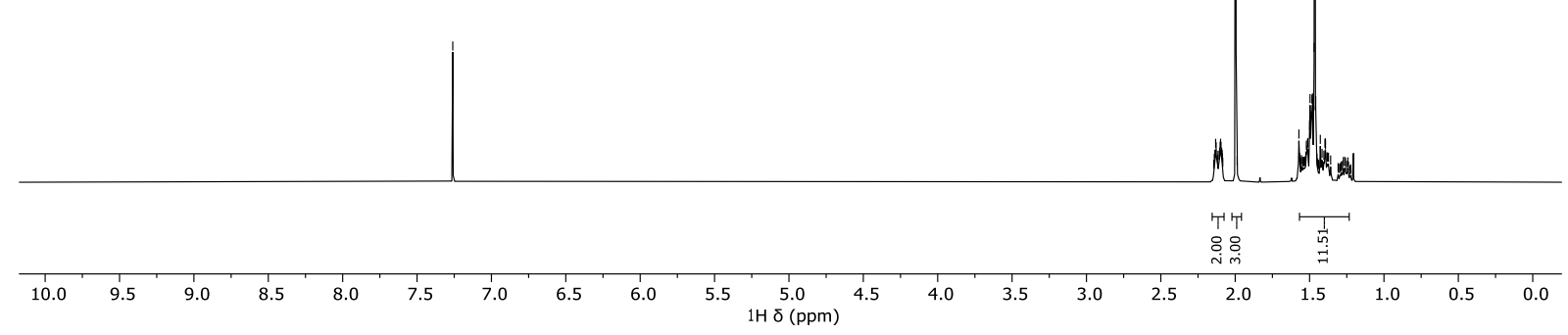

1-Methylcyclohexyl acetate $-{ }^{13} \mathrm{C}\left\{{ }^{1} \mathrm{H}\right\} \mathrm{NMR}\left(101 \mathrm{MHz}, \mathrm{CDCl}_{3}\right)$

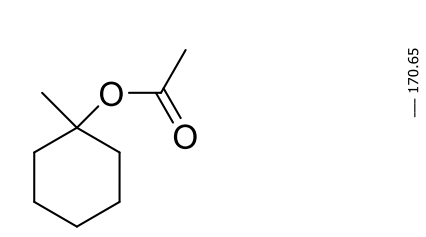

$\begin{array}{lllllllllll}220 & 210 & 200 & 190 & 180 & 170 & 160 & 150 & 140 & 130 & 120 \\ 13 \mathrm{C} \delta(\mathrm{ppm}) & 100\end{array}$

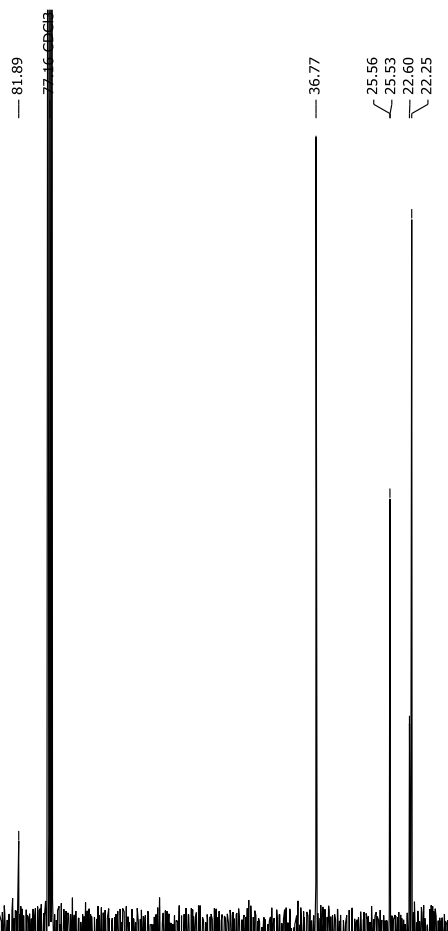

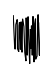

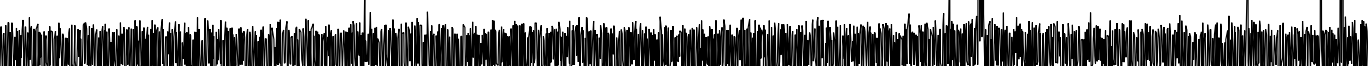

等
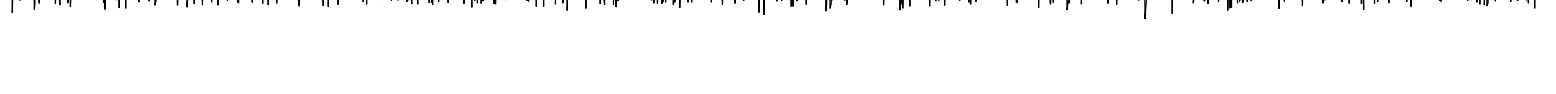
1-Phenylcyclohexyl acetate $-{ }^{1} \mathrm{H}$ NMR $\left(500 \mathrm{MHz}, \mathrm{CDCl}_{3}\right)$

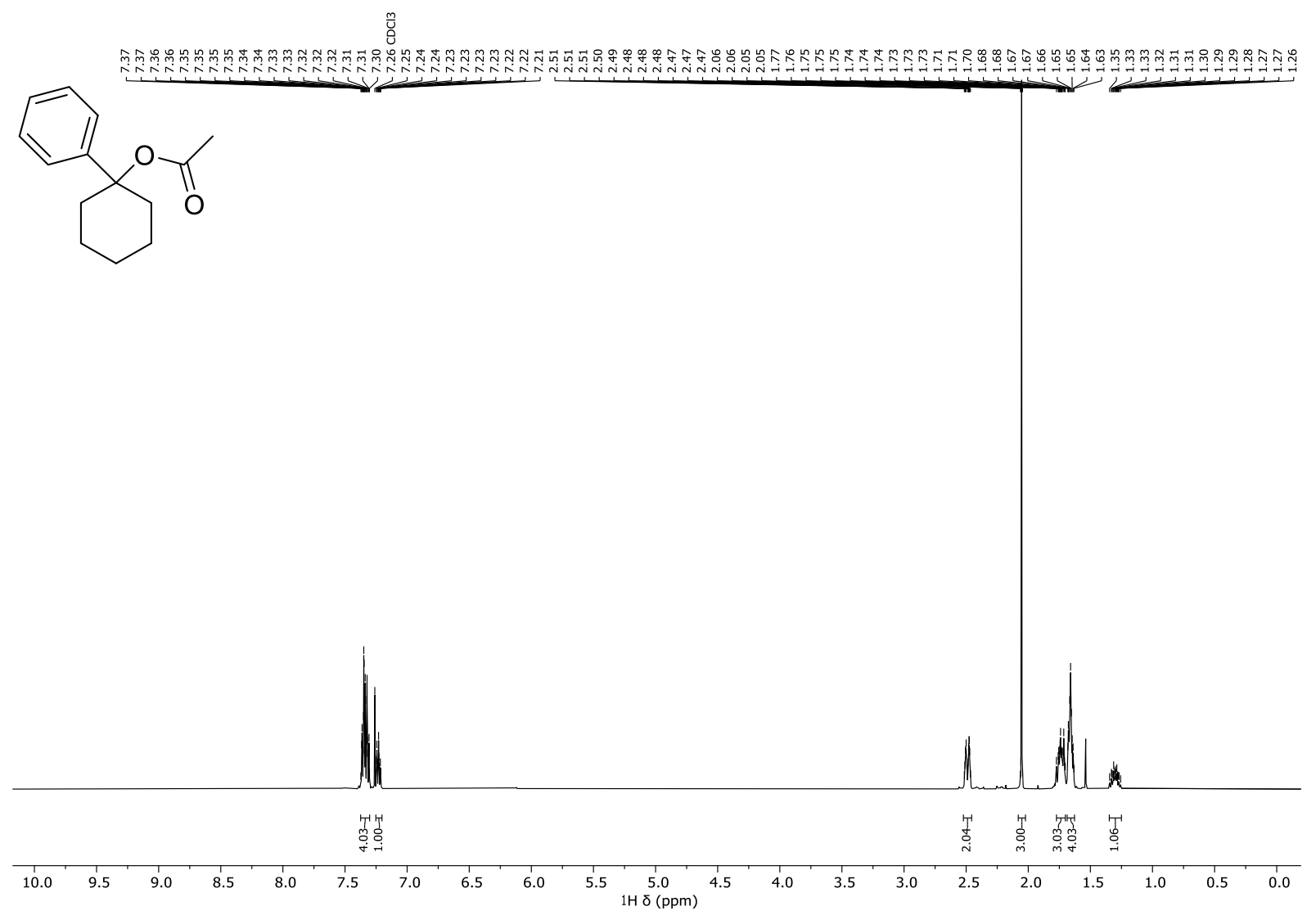

1-Phenylcyclohexyl acetate $-{ }^{13} \mathrm{C}\left\{{ }^{1} \mathrm{H}\right\} \mathrm{NMR}\left(126 \mathrm{MHz}, \mathrm{CDCl}_{3}\right)$
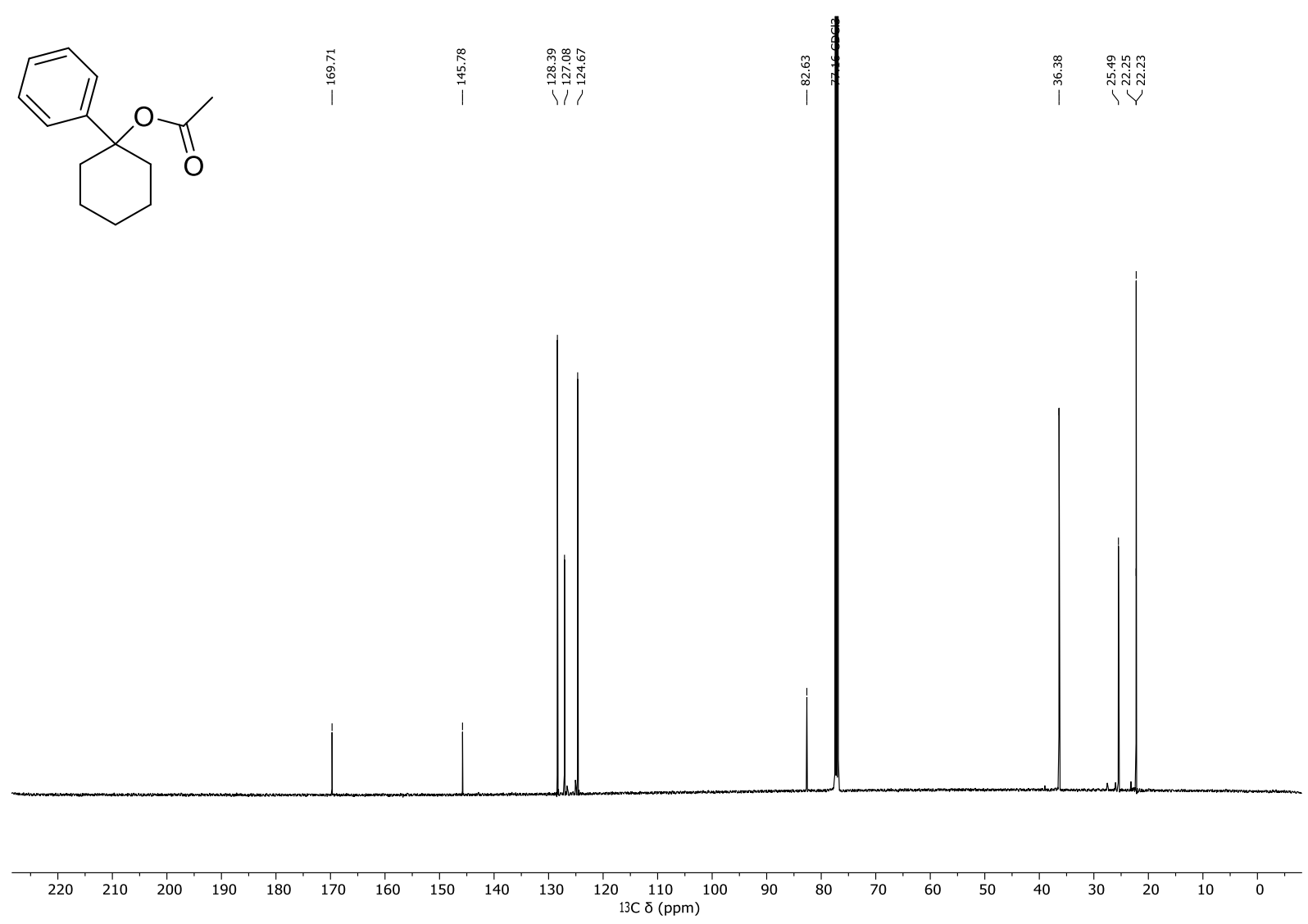

S104 
1-Adamantyl acetate - ${ }^{1} \mathrm{H} \mathrm{NMR}\left(400 \mathrm{MHz}, \mathrm{CDCl}_{3}\right)$
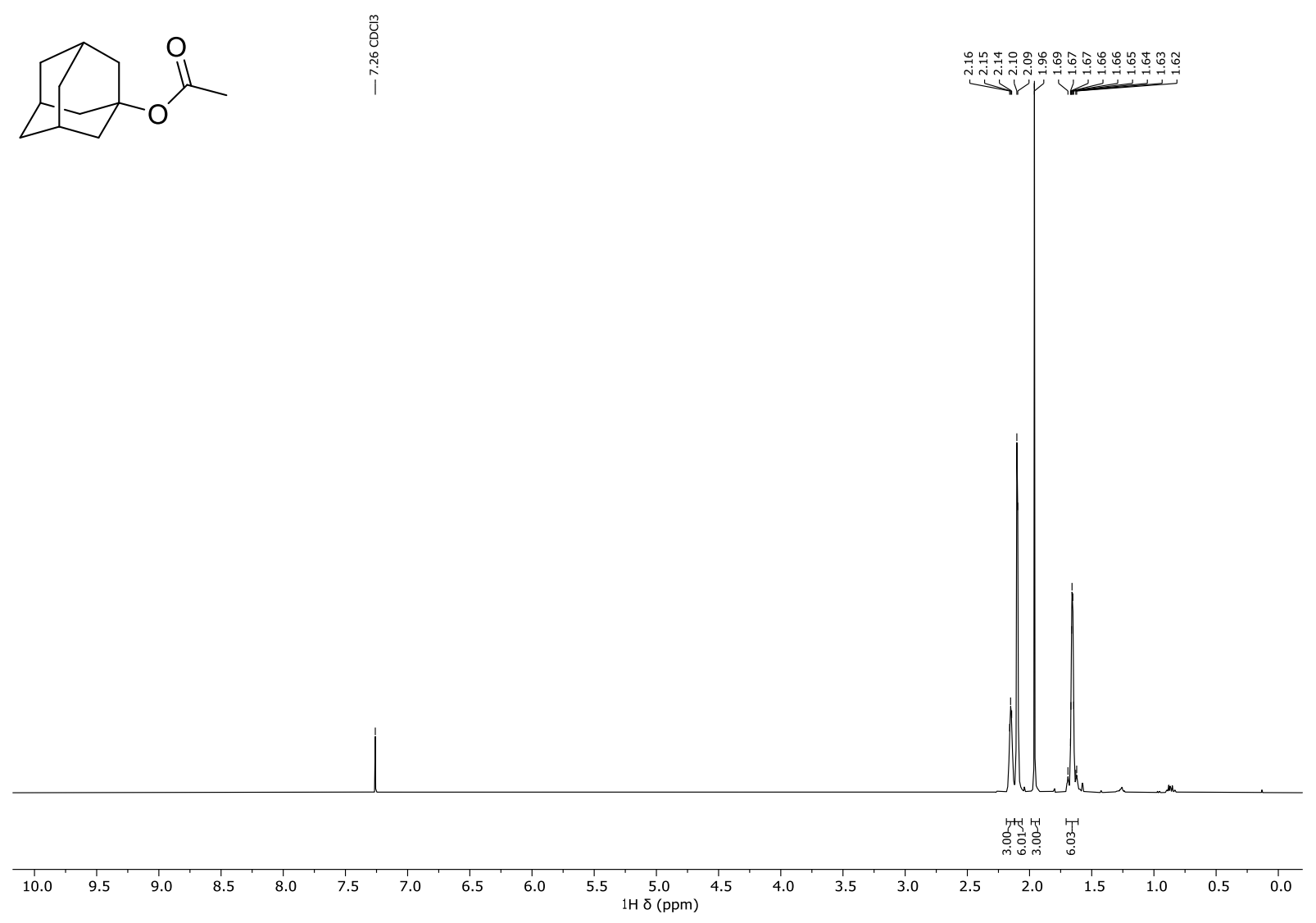

1-Adamantyl acetate $-{ }^{13} \mathrm{C}\left\{{ }^{1} \mathrm{H}\right\}$ NMR (101 MHz, $\left.\mathrm{CDCl}_{3}\right)$
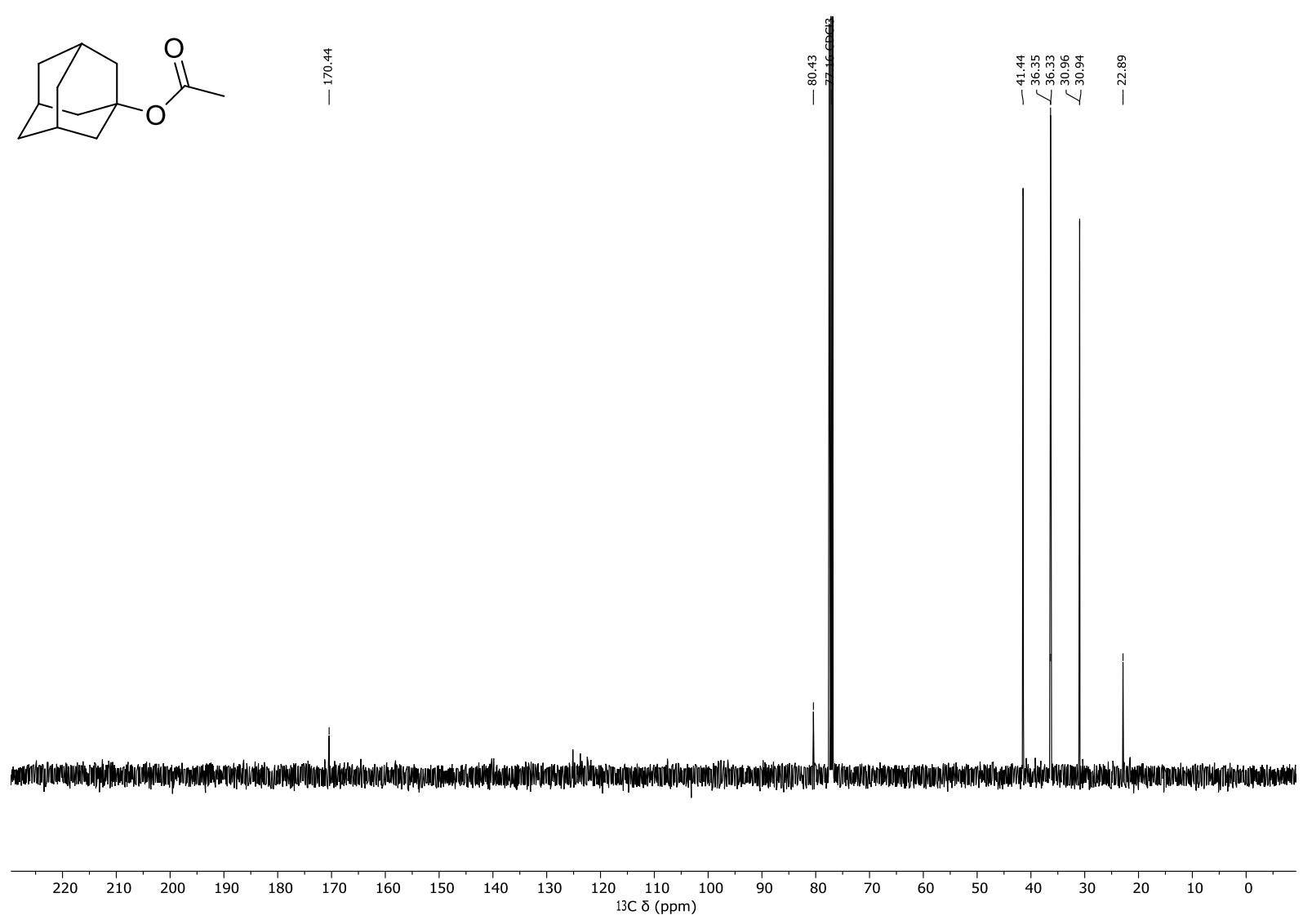

S105 


$$
=
$$


1-Adamantyl benzoate - ${ }^{1} \mathrm{H}$ NMR $\left(400 \mathrm{MHz}, \mathrm{CDCl}_{3}\right)$

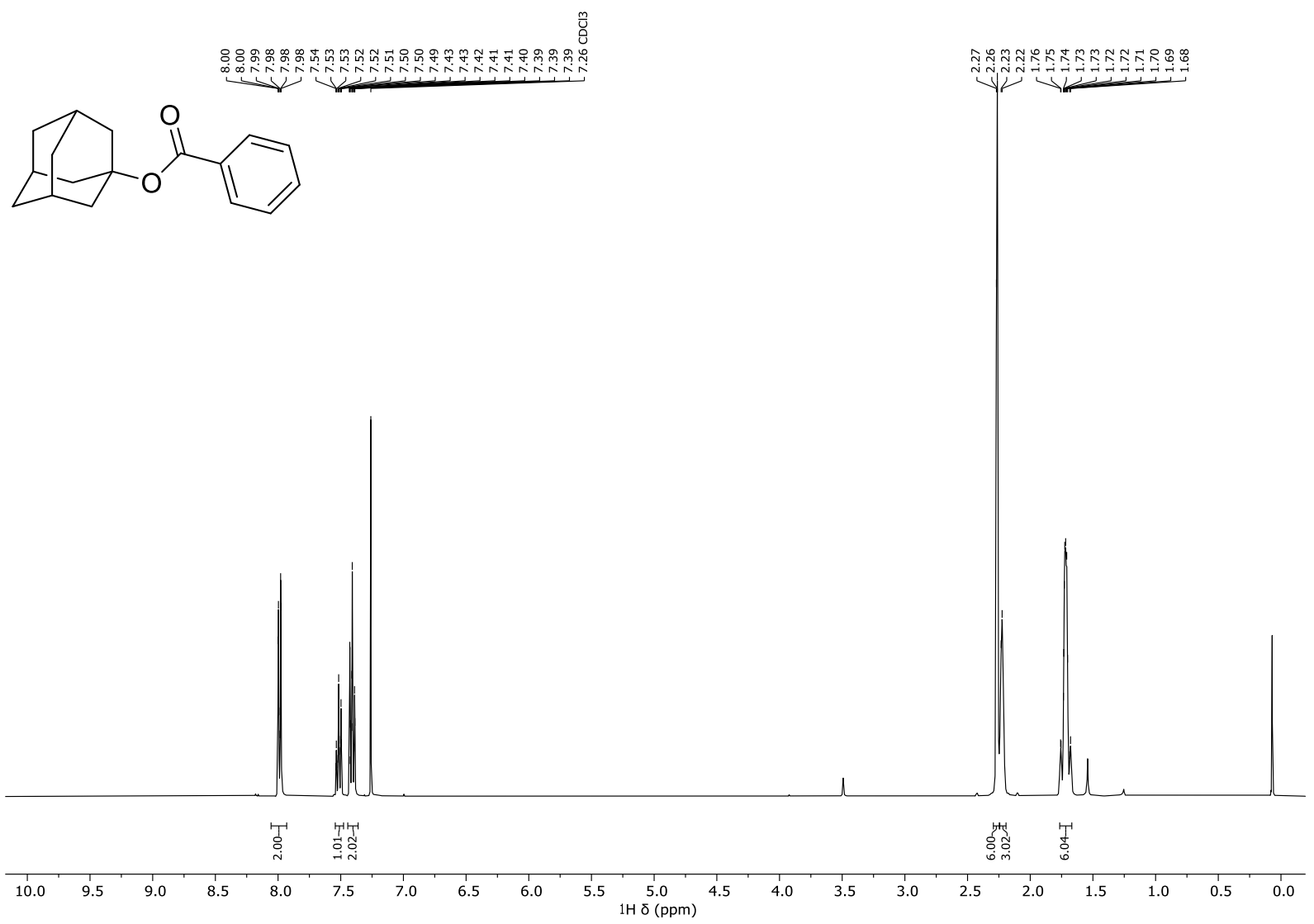

1-Adamantyl benzoate $-{ }^{13} \mathrm{C}\left\{{ }^{1} \mathrm{H}\right\} \mathrm{NMR}\left(101 \mathrm{MHz}, \mathrm{CDCl}_{3}\right)$<smiles>O=C(OC12CC3CC(CC(C3)C1)C2)c1ccccc1</smiles>

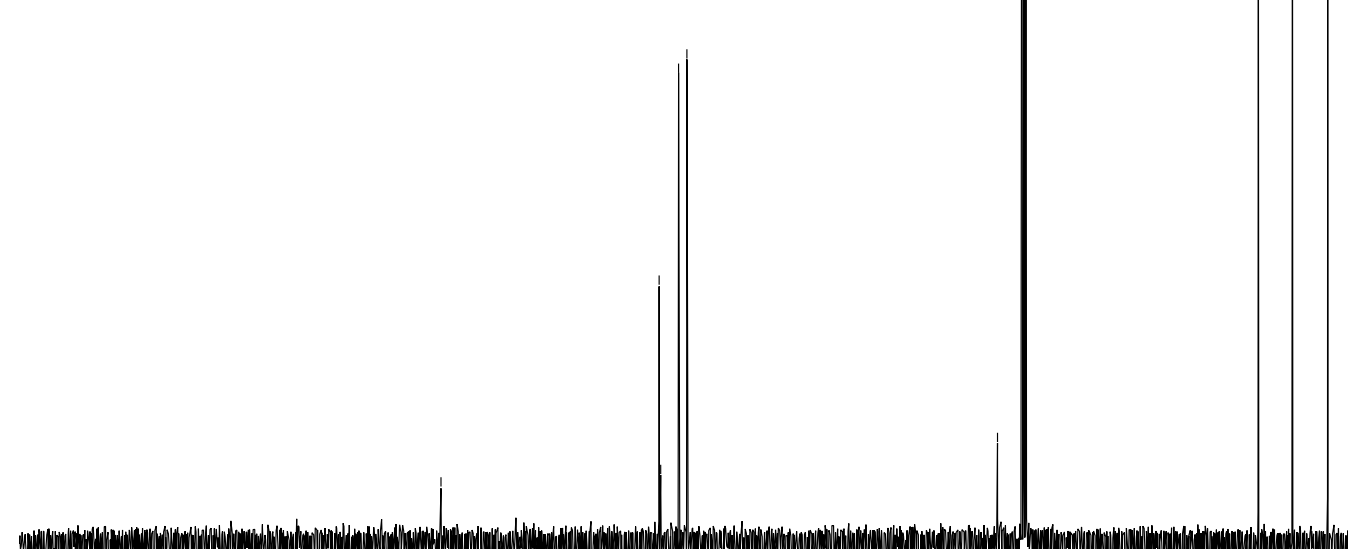

(1)

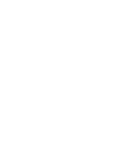

$\begin{array}{lllllllllll}220 & 210 & 200 & 190 & 180 & 170 & 160 & 150 & 140 & 130 & 120 \\ 13 \mathrm{C} \delta(\mathrm{ppm}) & 100\end{array}$ 
2-Methyl-2-adamantanol - ${ }^{1} \mathrm{H} \mathrm{NMR}\left(500 \mathrm{MHz}, \mathrm{CDCl}_{3}\right)$<smiles>CC1(O)C2CC3CC(C2)CC1C3</smiles>
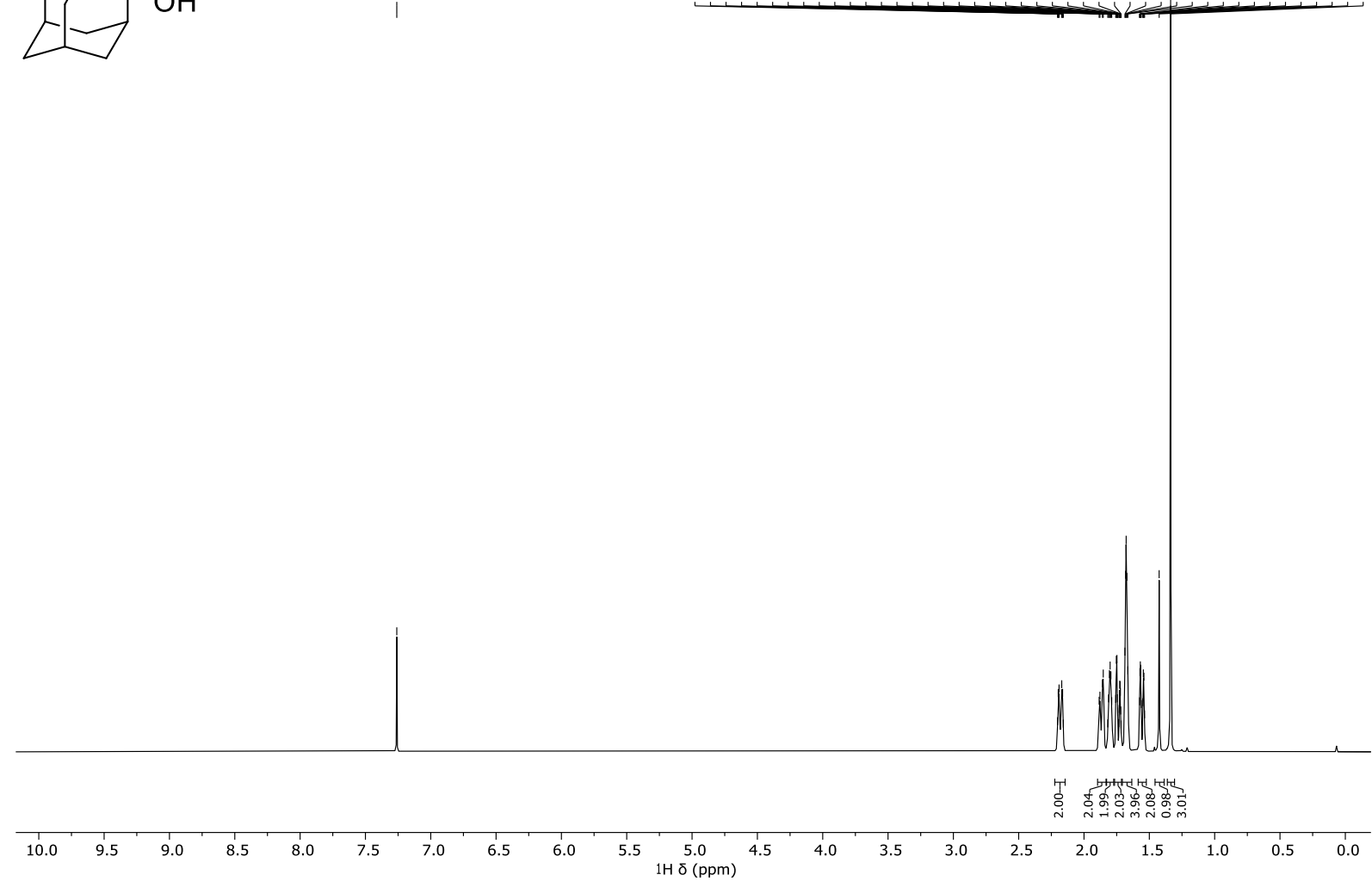

2-Methyl-2-adamantanol - ${ }^{13} \mathrm{C}\left\{{ }^{1} \mathrm{H}\right\} \mathrm{NMR}\left(126 \mathrm{MHz}, \mathrm{CDCl}_{3}\right)$<smiles>CC1(O)C2CC3CC(C2)CC1C3</smiles> 
2-Methyl-2-adamantyl acetate - ${ }^{1} \mathrm{H}$ NMR $\left(500 \mathrm{MHz}, \mathrm{CDCl}_{3}\right)$
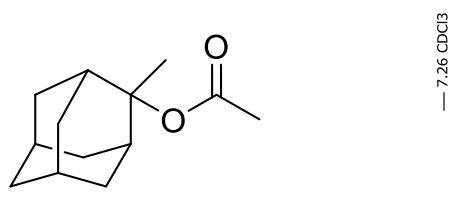

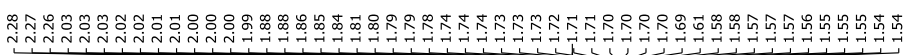

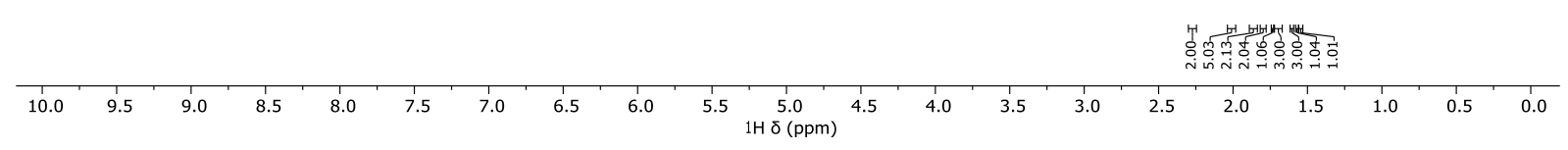

2-Methyl-2-adamantyl acetate $-{ }^{13} \mathrm{C}\left\{{ }^{1} \mathrm{H}\right\}$ NMR $\left(126 \mathrm{MHz}, \mathrm{CDCl}_{3}\right)$
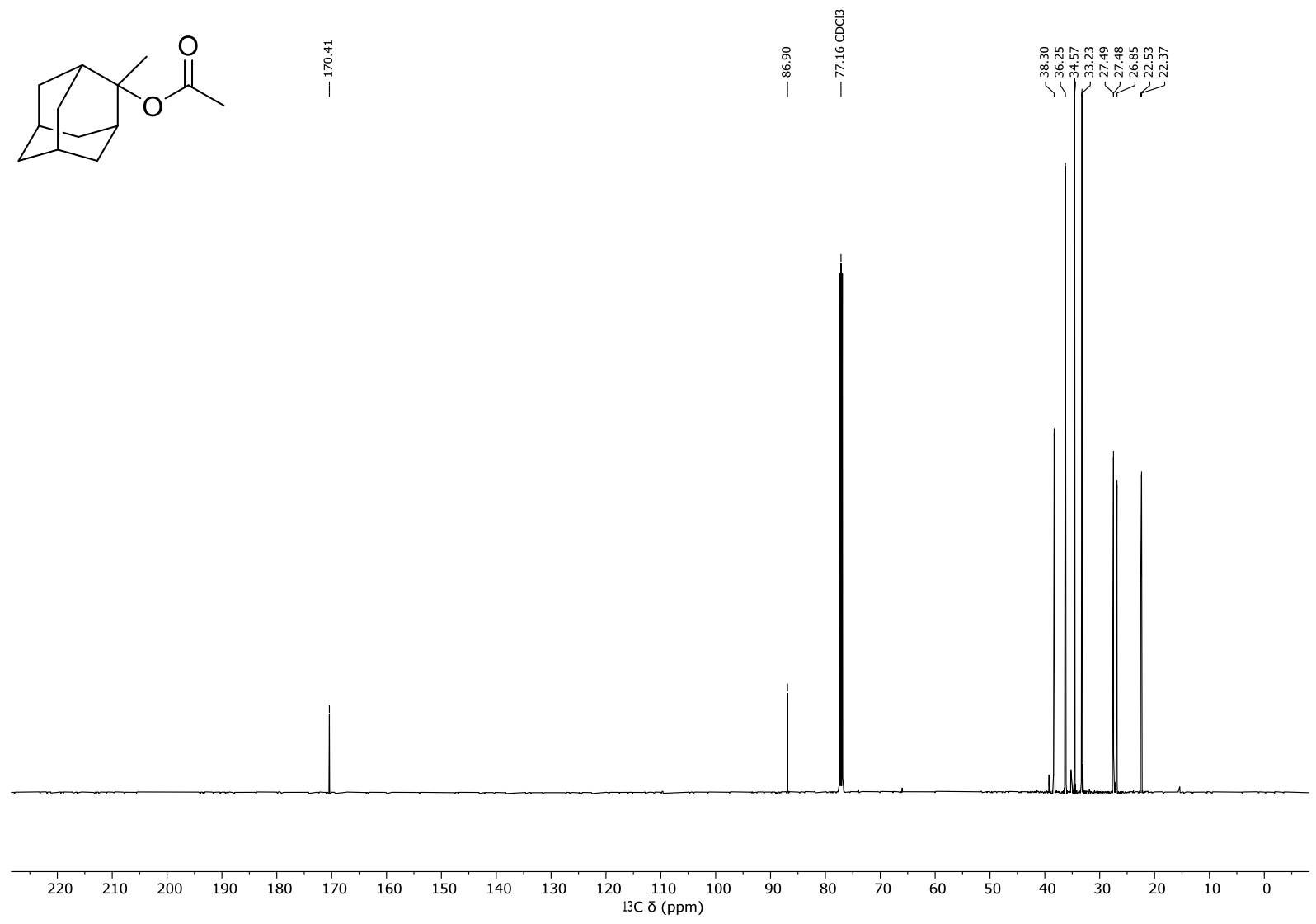

S109 
(+)-Cedryl acetate - ${ }^{1} \mathrm{H}$ NMR $\left(500 \mathrm{MHz}, \mathrm{CDCl}_{3}\right)$

范

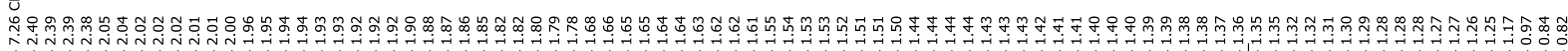<smiles>CC(=O)O[C@]1(C)CC[C@]23CC[C@]2(CCC[C@@H]3C)C1(C)C</smiles>

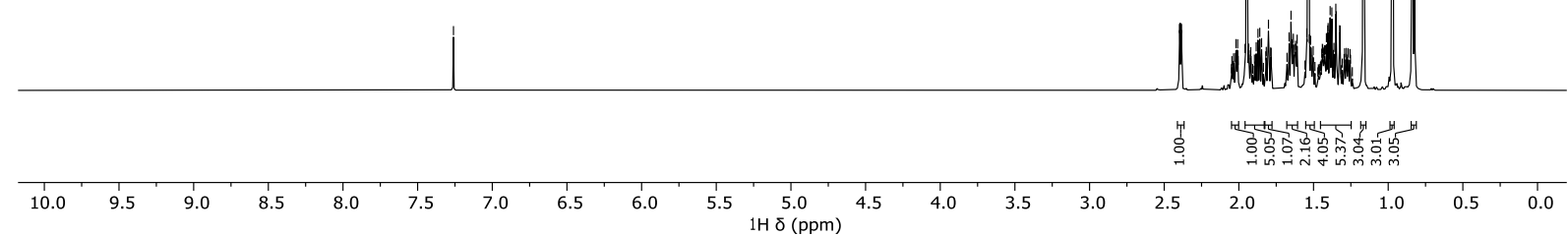

(+)-Cedryl acetate $-{ }^{13} \mathrm{C}\left\{{ }^{1} \mathrm{H}\right\}$ NMR (126 MHz, $\left.\mathrm{CDCl}_{3}\right)$
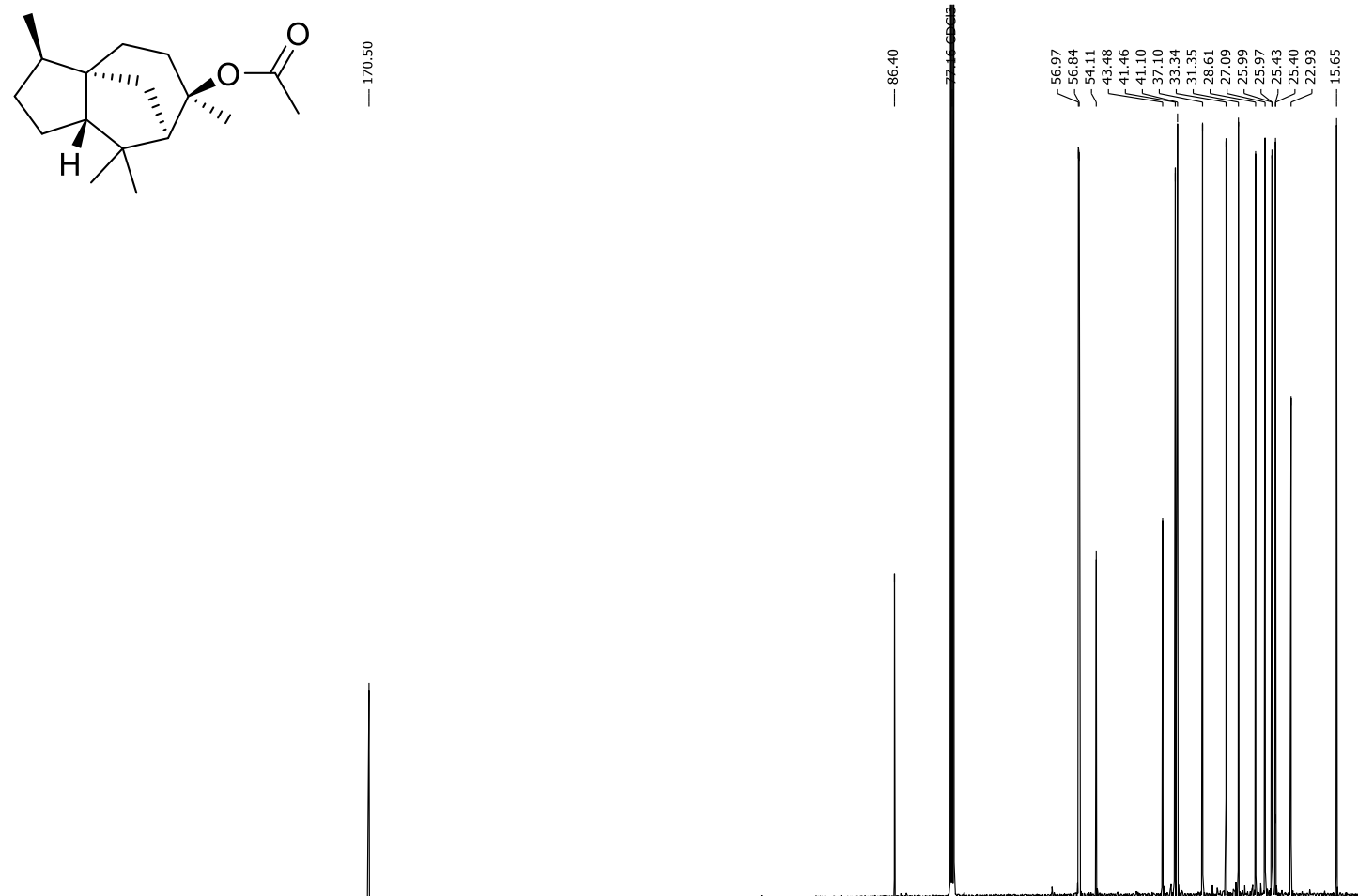

$\begin{array}{lllllllllll}220 & 210 & 200 & 190 & 180 & 170 & 160 & 150 & 140 & 130 & 120 \\ 13 \mathrm{C} \delta(\mathrm{ppm}) & 100\end{array}$ 
2-Methyl-4-phenyl-2-butanol - ${ }^{1} \mathrm{H}$ NMR (500 $\mathrm{MHz}, \mathrm{CDCl}_{3}$ )

\section{要}

$\overbrace{\mathrm{Ph}}$

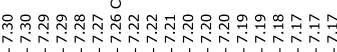

(1)

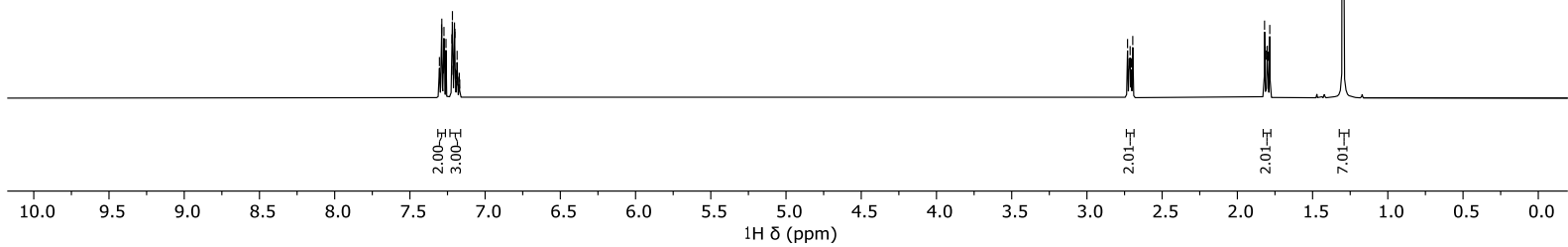

2-Methyl-4-phenyl-2-butanol - ${ }^{13} \mathrm{C}\left\{{ }^{1} \mathrm{H}\right\}$ NMR $\left(126 \mathrm{MHz}, \mathrm{CDCl}_{3}\right)$<smiles>CC(C)(O)CCc1ccccc1</smiles>
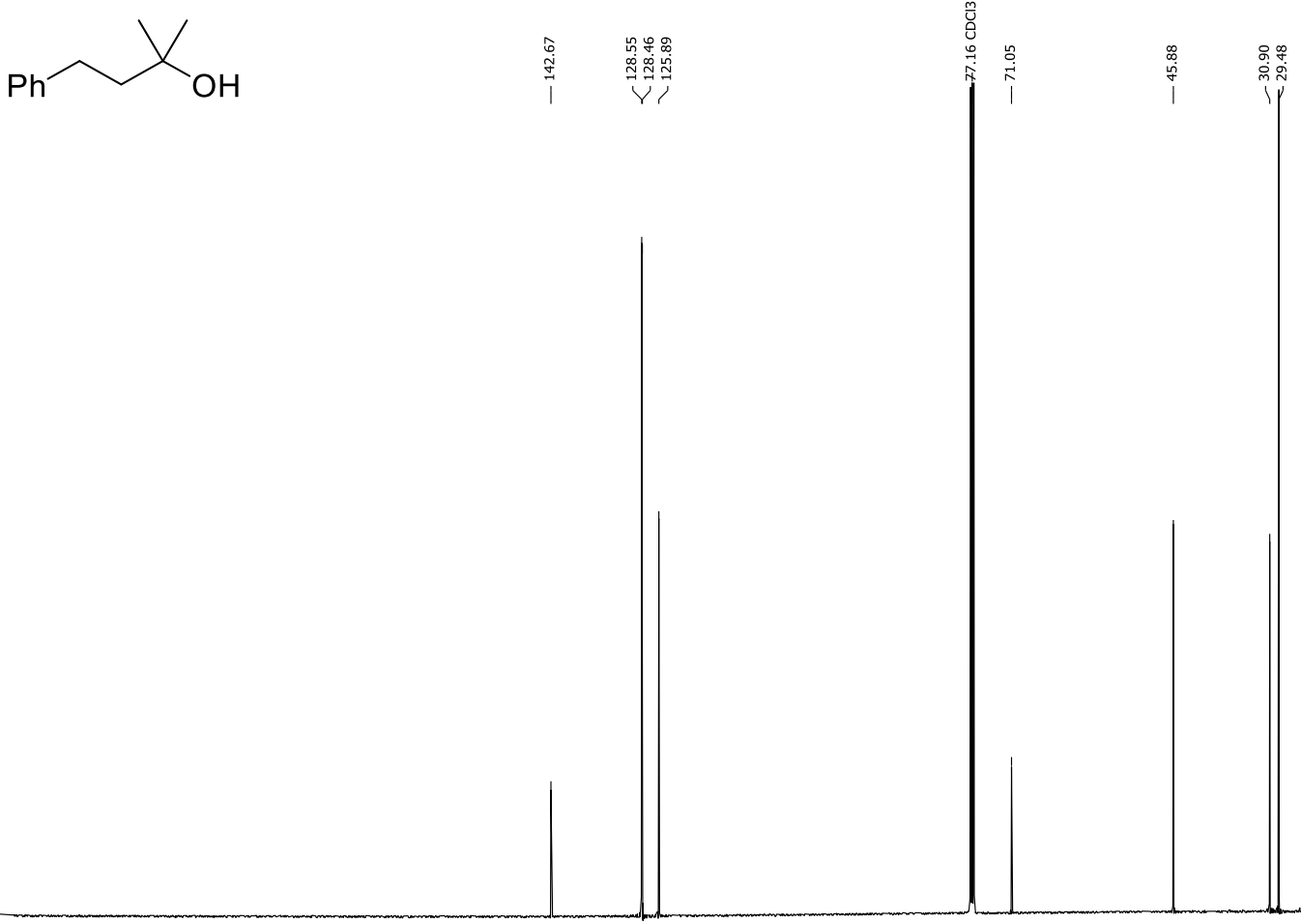

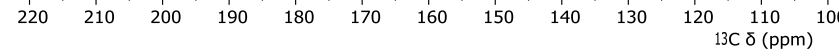


2-Methyl-4-phenyl-2-butyl acetate $\left(d_{0}-2\right)-{ }^{1} \mathrm{H}$ NMR $\left(500 \mathrm{MHz}, \mathrm{CDCl}_{3}\right)$
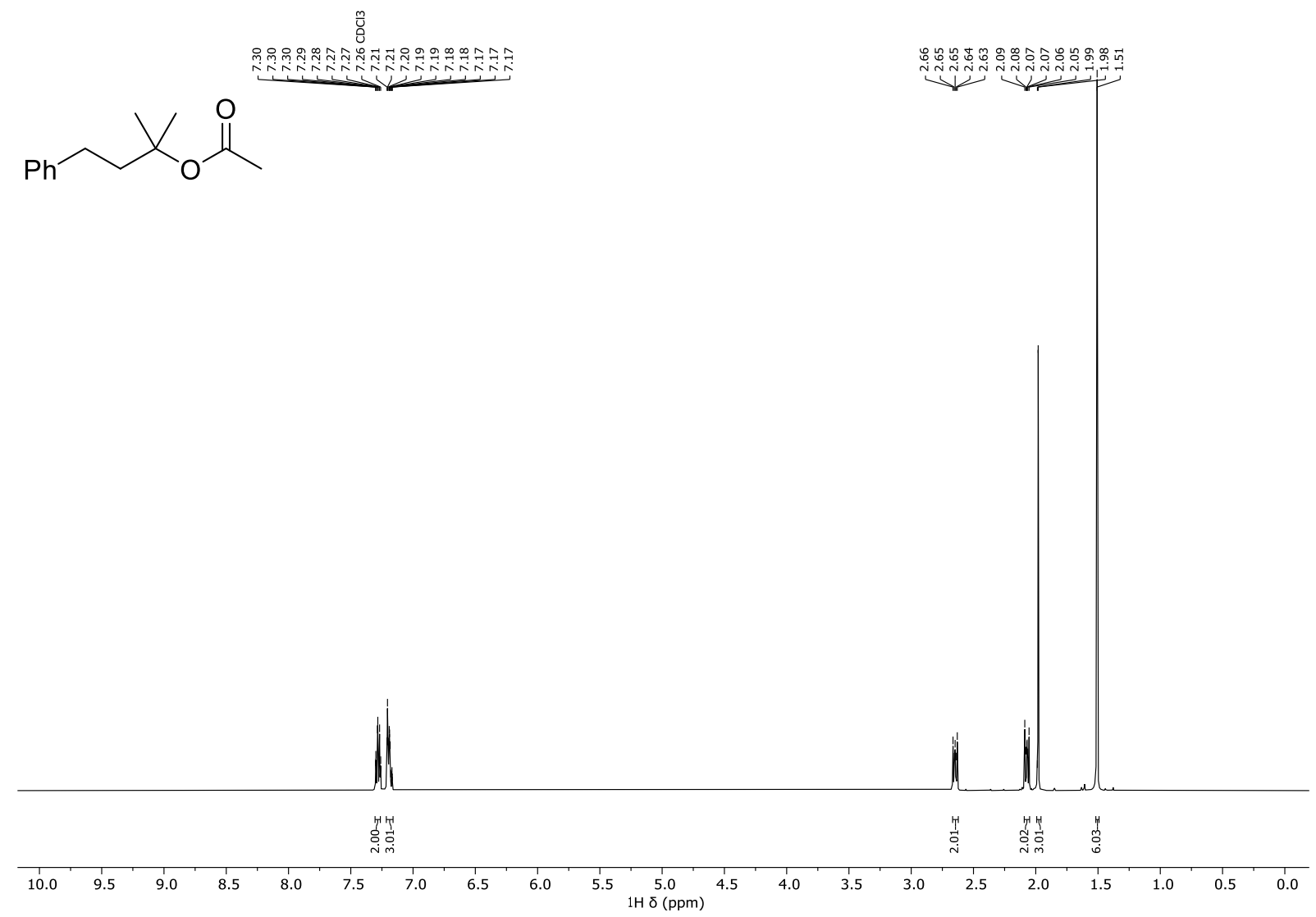

2-Methyl-4-phenyl-2-butyl acetate $\left(d_{0}-2\right)-{ }^{13} \mathrm{C}\left\{{ }^{1} \mathrm{H}\right\} \mathrm{NMR}\left(126 \mathrm{MHz}, \mathrm{CDCl}_{3}\right)$
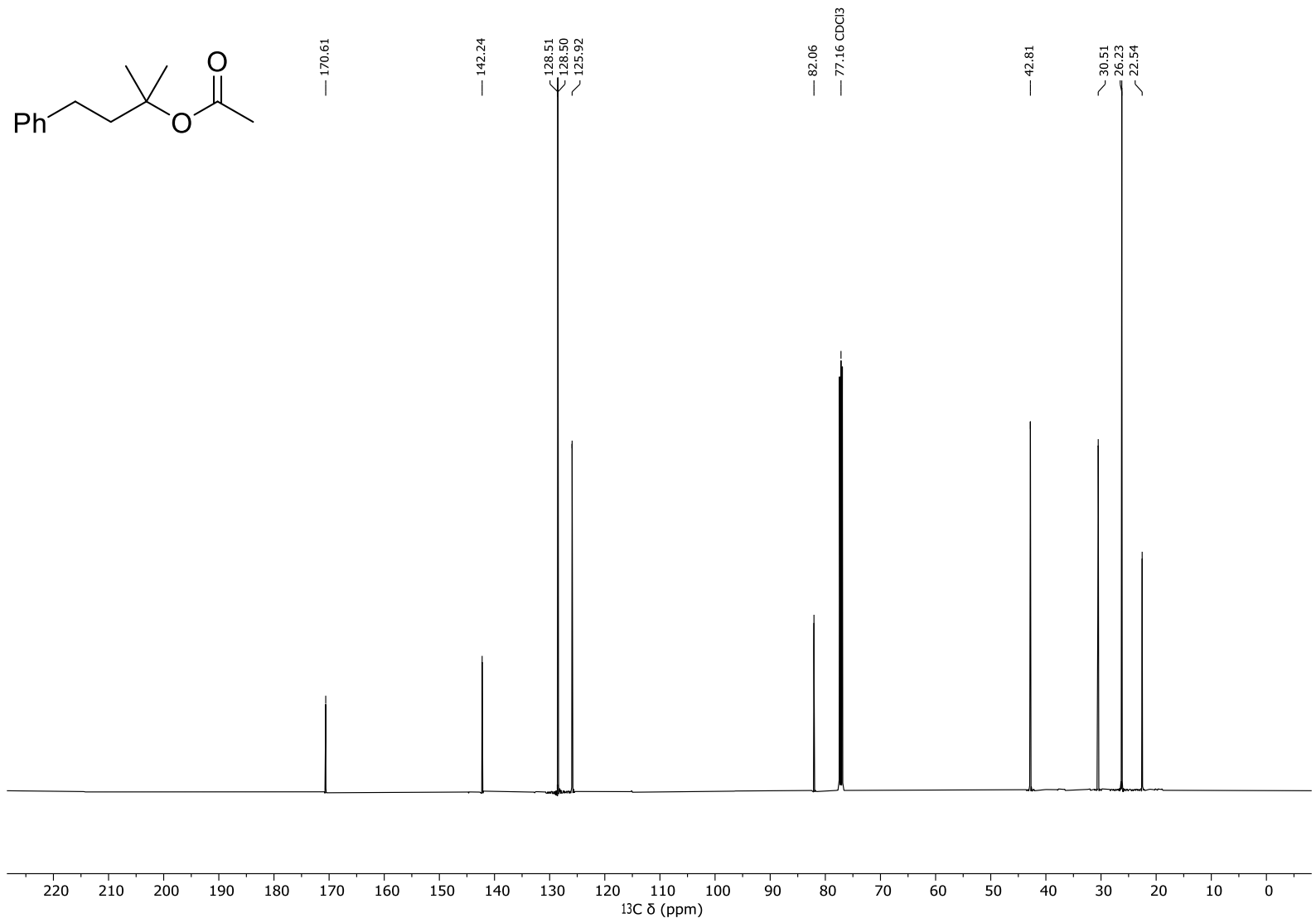
2-Methyl-4-phenyl-2-butanol- $d_{6}-{ }^{1} \mathrm{H}$ NMR $\left(500 \mathrm{MHz}, \mathrm{CDCl}_{3}\right)$

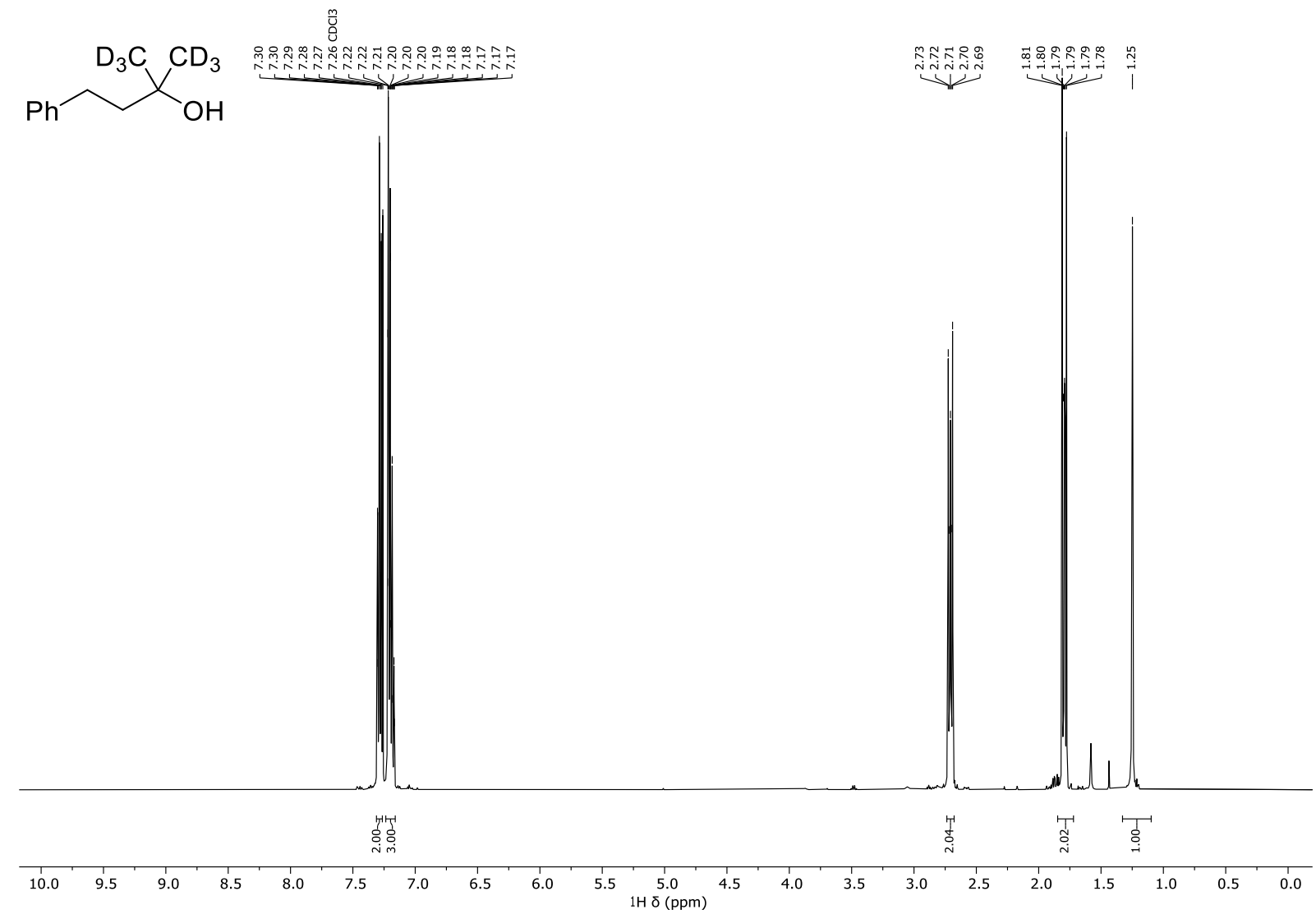

2-Methyl-4-phenyl-2-butanol- $d_{6}-{ }^{13} \mathrm{C}\left\{{ }^{1} \mathrm{H}\right\}$ NMR (126 MHz, $\left.\mathrm{CDCl}_{3}\right)$

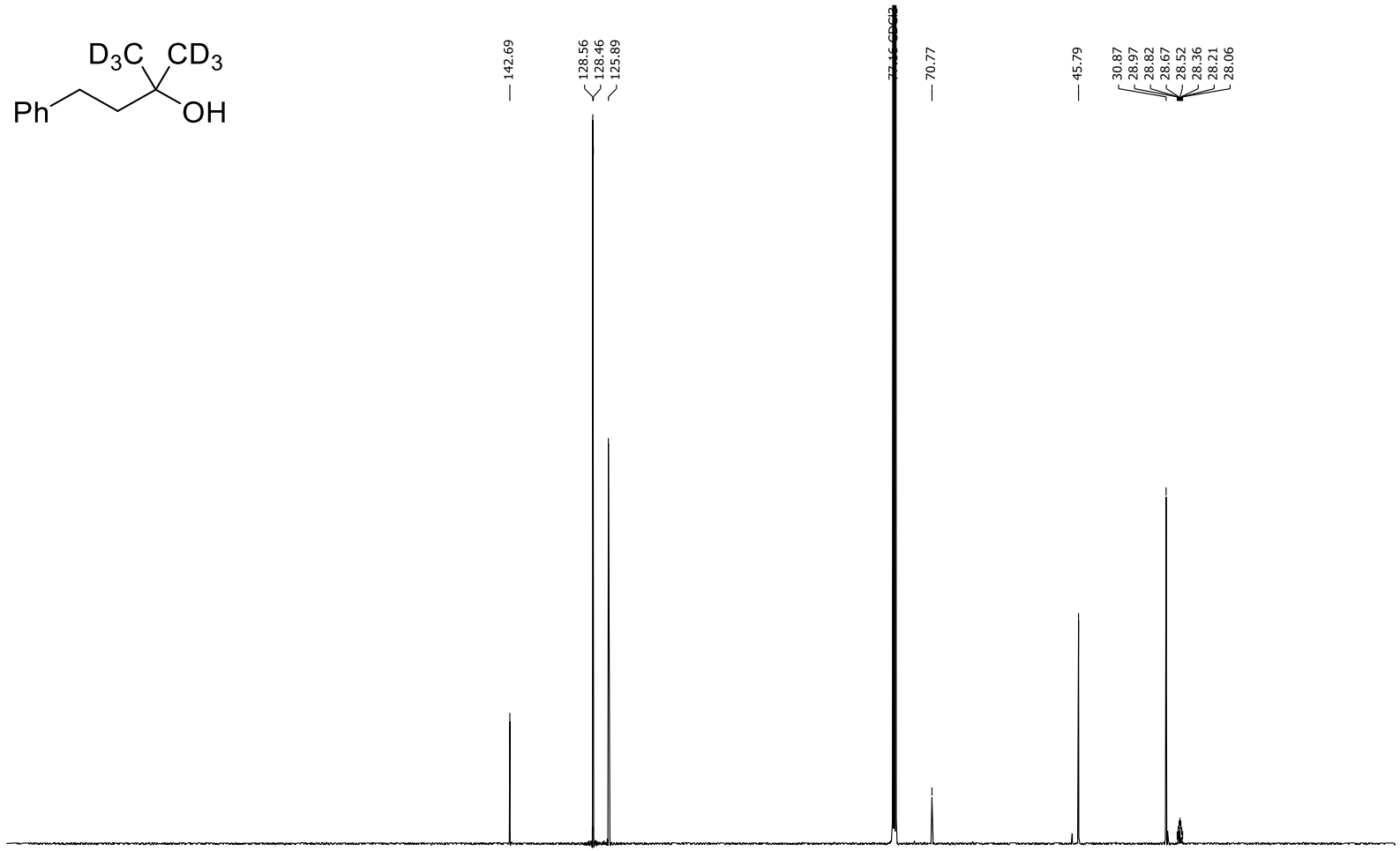

$\begin{array}{llllllllllllllllllllllll}220 & 210 & 200 & 190 & 180 & 170 & 160 & 150 & 140 & 130 & 120 & 110 & 100 & 90 & 80 & 70 & 60 & 50 & 40 & 30 & 20 & 10 & 0\end{array}$ 
2-Methyl-4-phenyl-2-butyl acetate- $d_{6}\left(d_{6}-2\right)-{ }^{1} \mathrm{H} N M R\left(500 \mathrm{MHz}, \mathrm{CDCl}_{3}\right)$
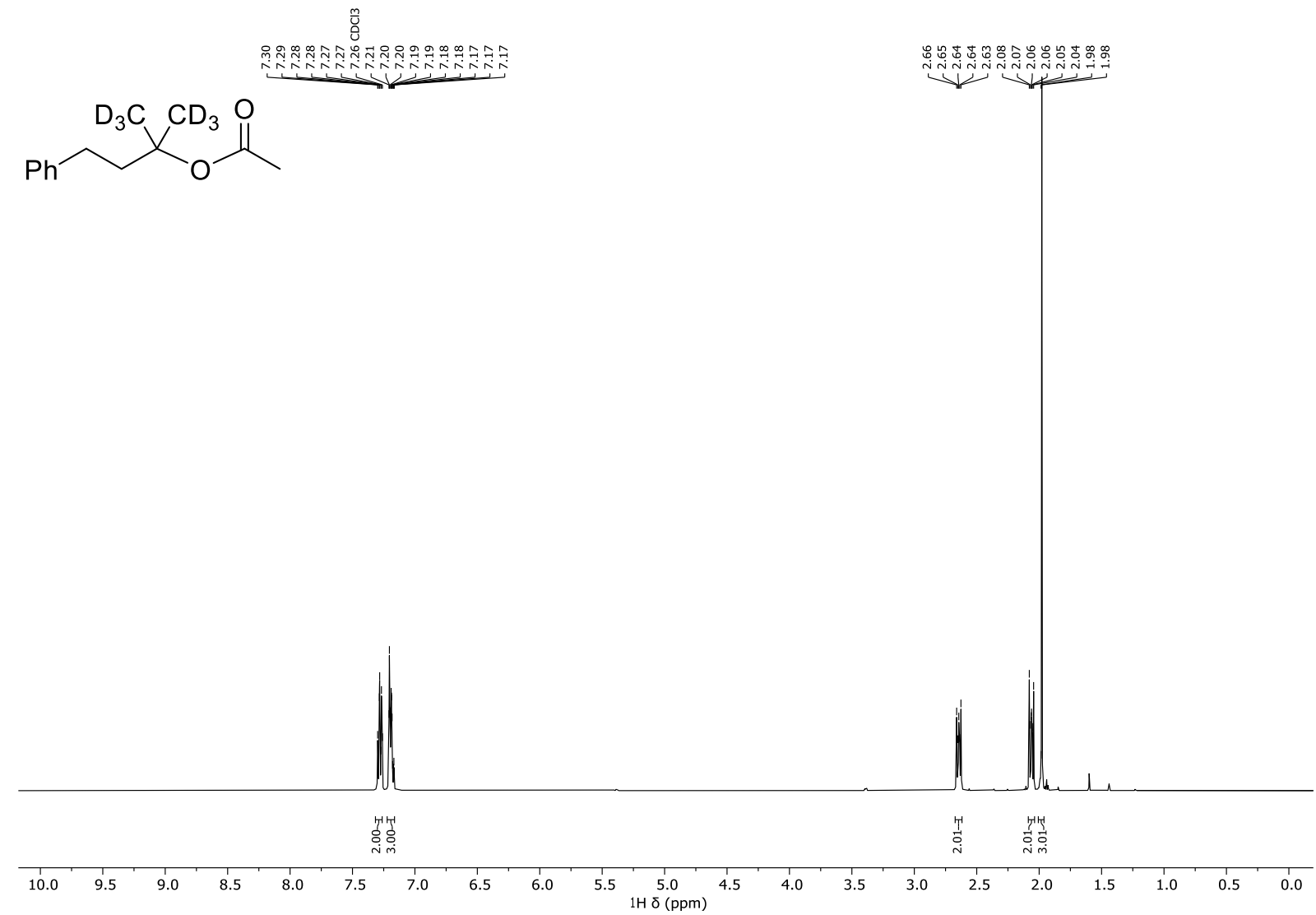

2-Methyl-4-phenyl-2-butyl acetate- $d_{6}\left(d_{6}-2\right)-{ }^{13} \mathrm{C}\left\{{ }^{1} \mathrm{H}\right\} \mathrm{NMR}\left(126 \mathrm{MHz}, \mathrm{CDCl}_{3}\right)$

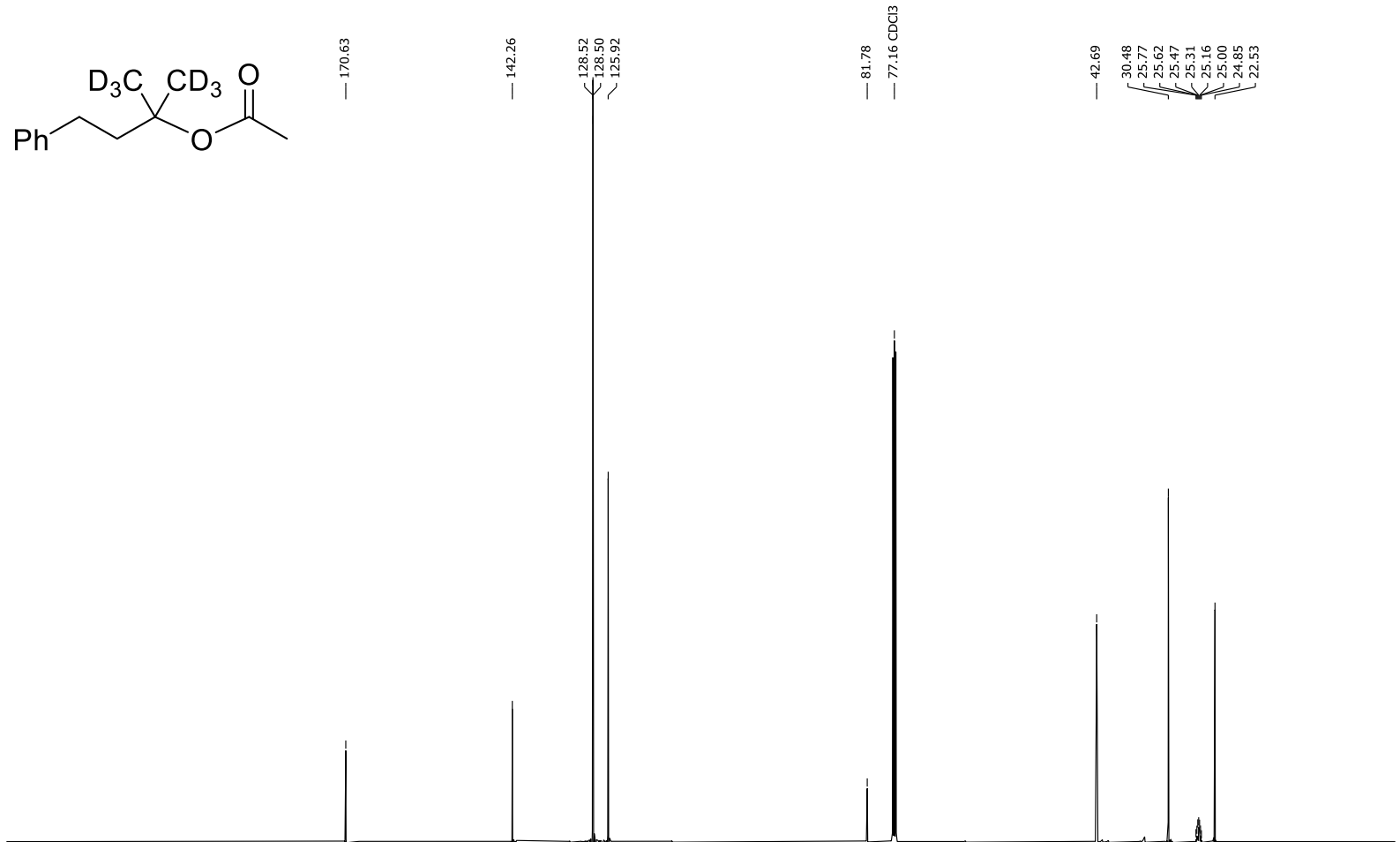

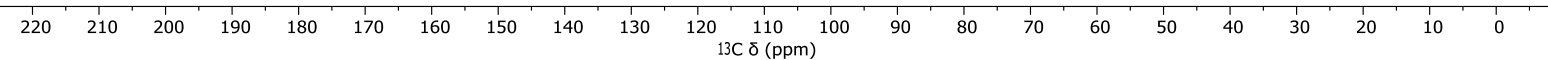


Di-tert-amylphosphonium triflate (1a) - ${ }^{1} \mathrm{H}$ NMR (500 MHz, $\left.\mathrm{CDCl}_{3}\right)$

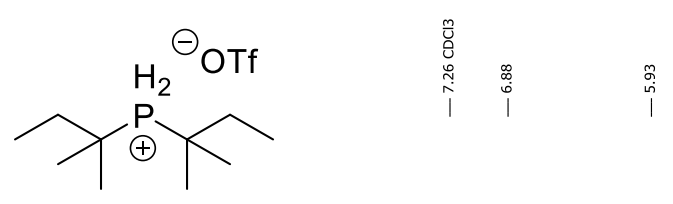

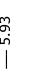

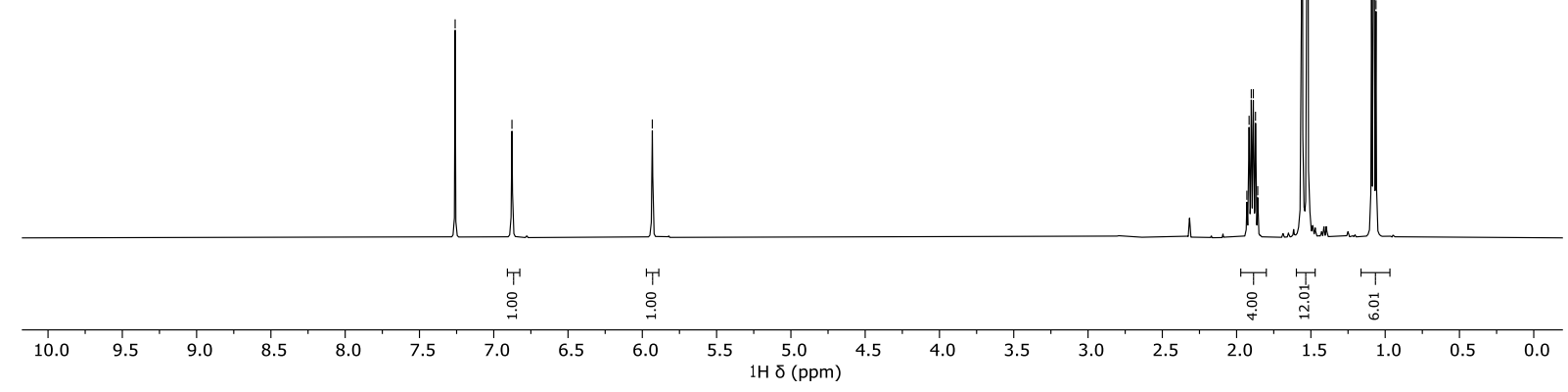

Di-tert-amylphosphonium triflate (1a) - ${ }^{13} \mathrm{C}\left\{{ }^{1} \mathrm{H}\right\}$ NMR (126 MHz, $\left.\mathrm{CDCl}_{3}\right)$<smiles>CCC(C)(C)PC(C)(C)CC</smiles> 
Di-tert-amylphosphonium triflate (1a) $\left.{ }^{19} \mathrm{~F} \mathrm{NMR} \mathrm{(470} \mathrm{MHz,} \mathrm{CDCl}_{3}\right)$<smiles>CCC(C)(C)PC(C)(C)CC</smiles>

\begin{tabular}{llllllllllllllllllllllllllll}
\hline 20 & 10 & 0 & -10 & -20 & -30 & -40 & -50 & -60 & -70 & -80 & -90 & -100 & -110 & -120 & -130 & -140 & -150 & -160 & -170 & -180 & -190 & -200 & -210 & $-2:$
\end{tabular}

Di-tert-amylphosphonium triflate (1a) ${ }^{31} \mathrm{P}\left\{{ }^{1} \mathrm{H}\right\} \mathrm{NMR}\left(202 \mathrm{MHz}, \mathrm{CDCl}_{3}\right)$

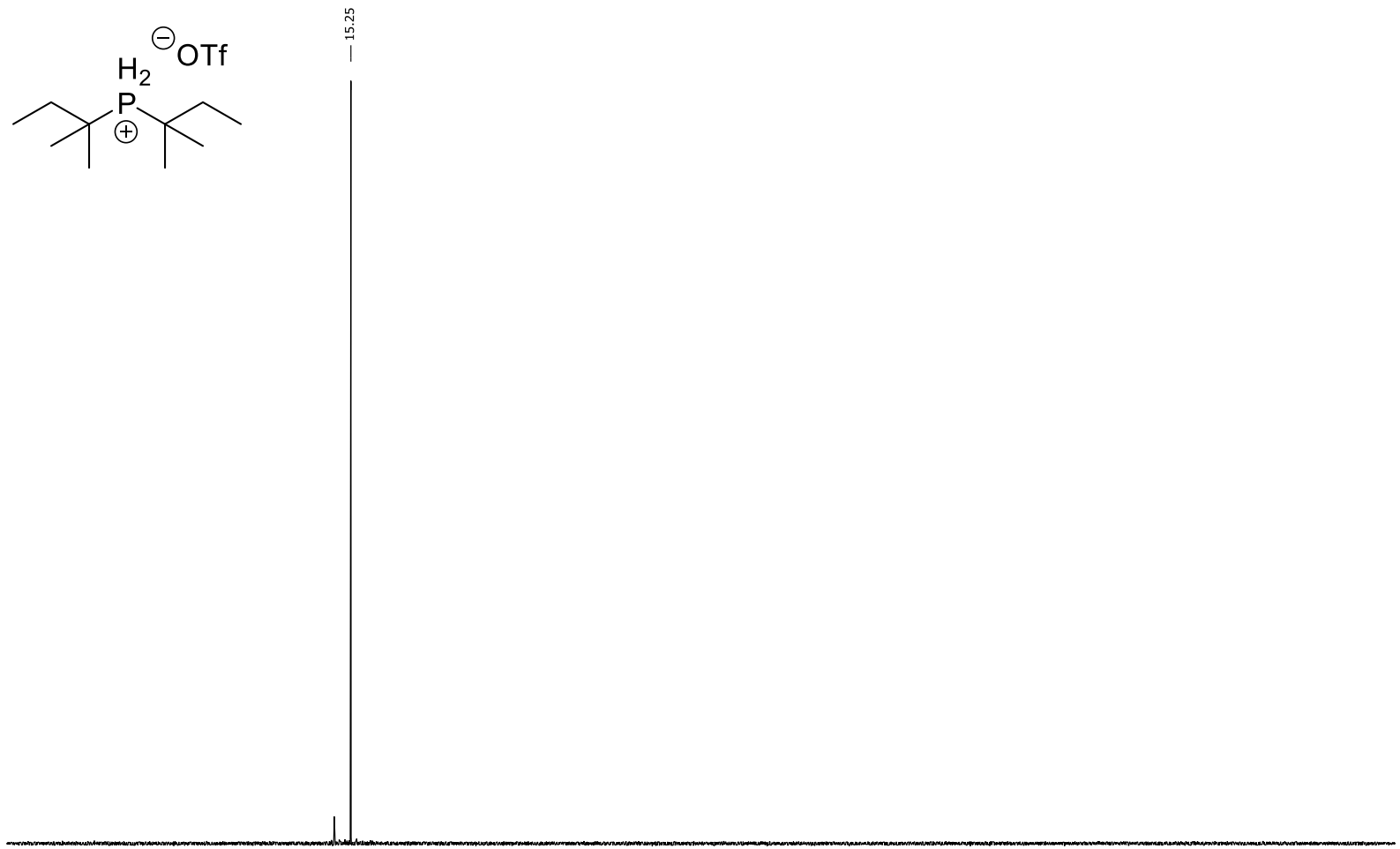

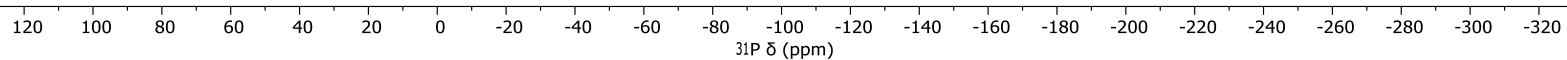


Di-tert-amylphosphonium triflate (1a) $\left.{ }^{31} \mathrm{P} \mathrm{NMR} \mathrm{(202} \mathrm{MHz,} \mathrm{CDCl}_{3}\right)$<smiles>CCC(C)(C)PC(C)(C)CC</smiles>

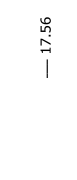<smiles>[13CH3]</smiles>

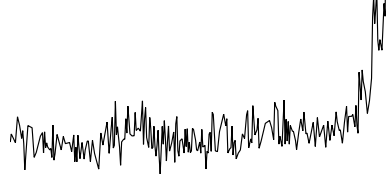<smiles>CCC</smiles>

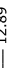

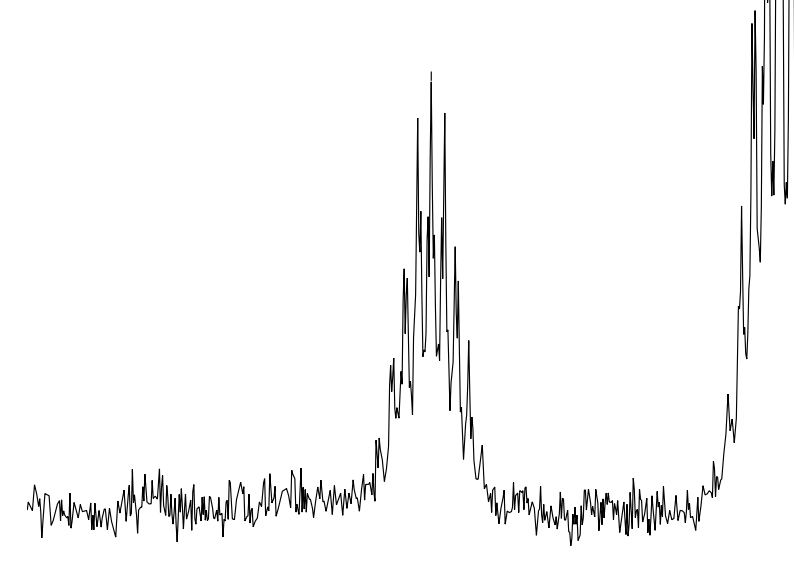

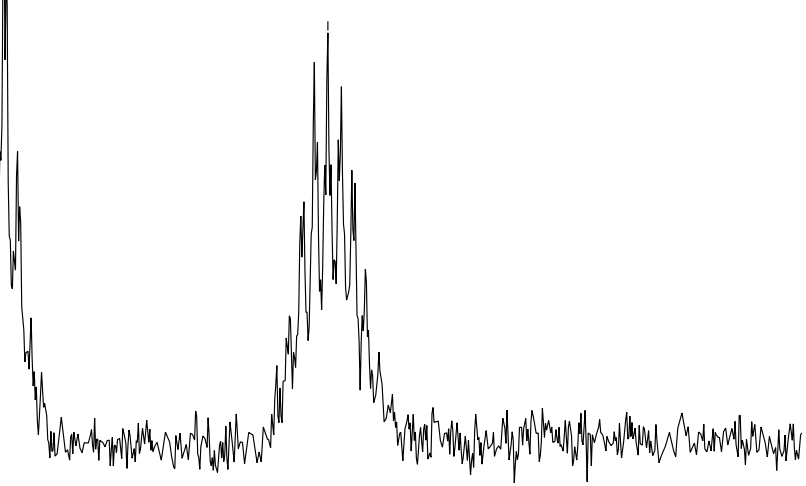

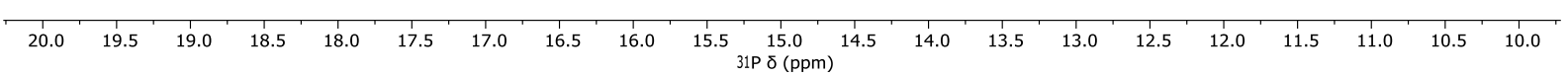


Di-(3-methyl-3-pentyl)phosphonium triflate (1b) - ${ }^{1} \mathrm{H} \mathrm{NMR}\left(500 \mathrm{MHz}, \mathrm{CDCl}_{3}\right)$<smiles>CCC(C)(CC)[Pb]C(CC)(CC)CC</smiles>
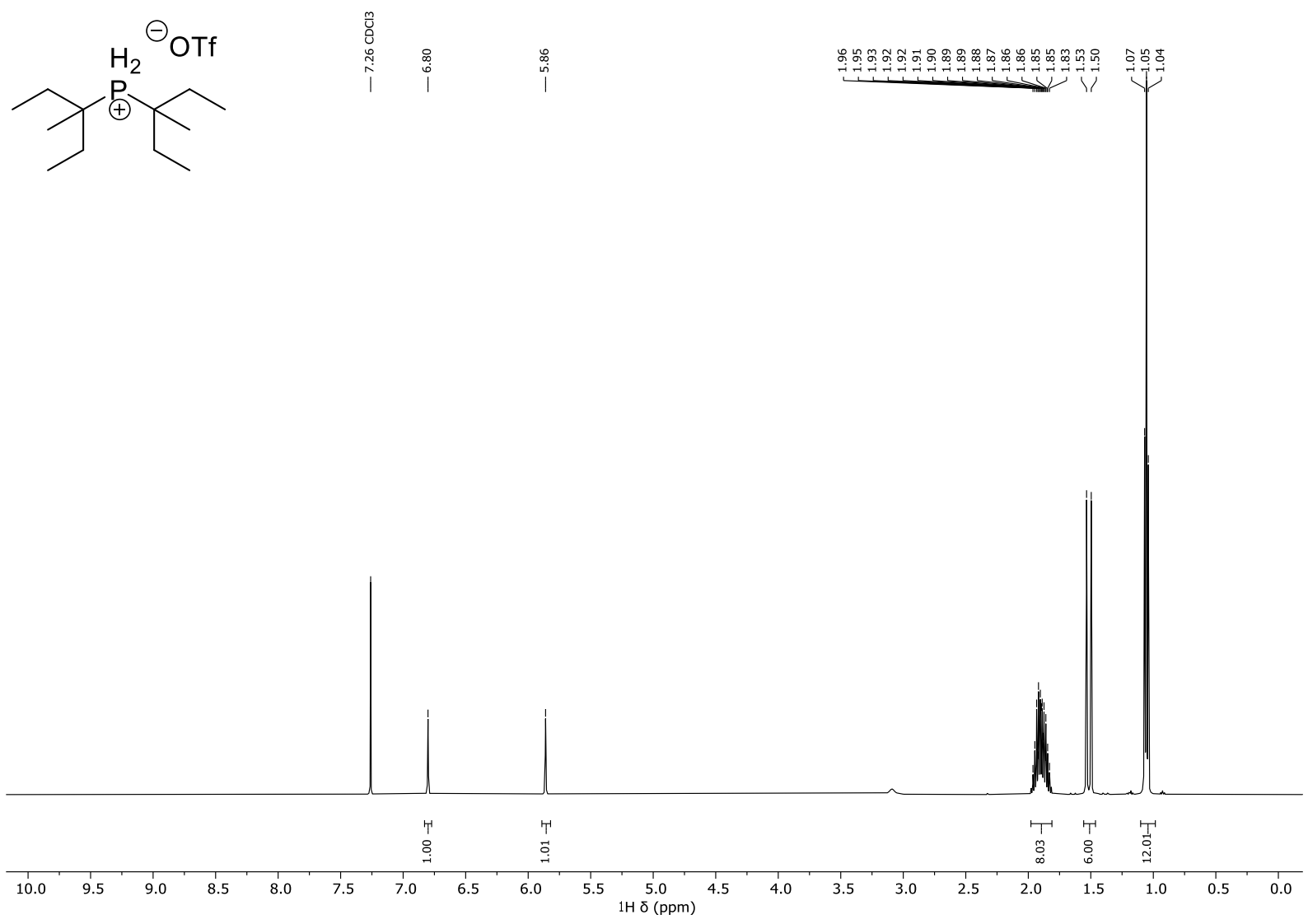

Di-(3-methyl-3-pentyl)phosphonium triflate (1b) - ${ }^{13} \mathrm{C}\left\{{ }^{1} \mathrm{H}\right\} \mathrm{NMR}\left(126 \mathrm{MHz}, \mathrm{CDCl}_{3}\right)$

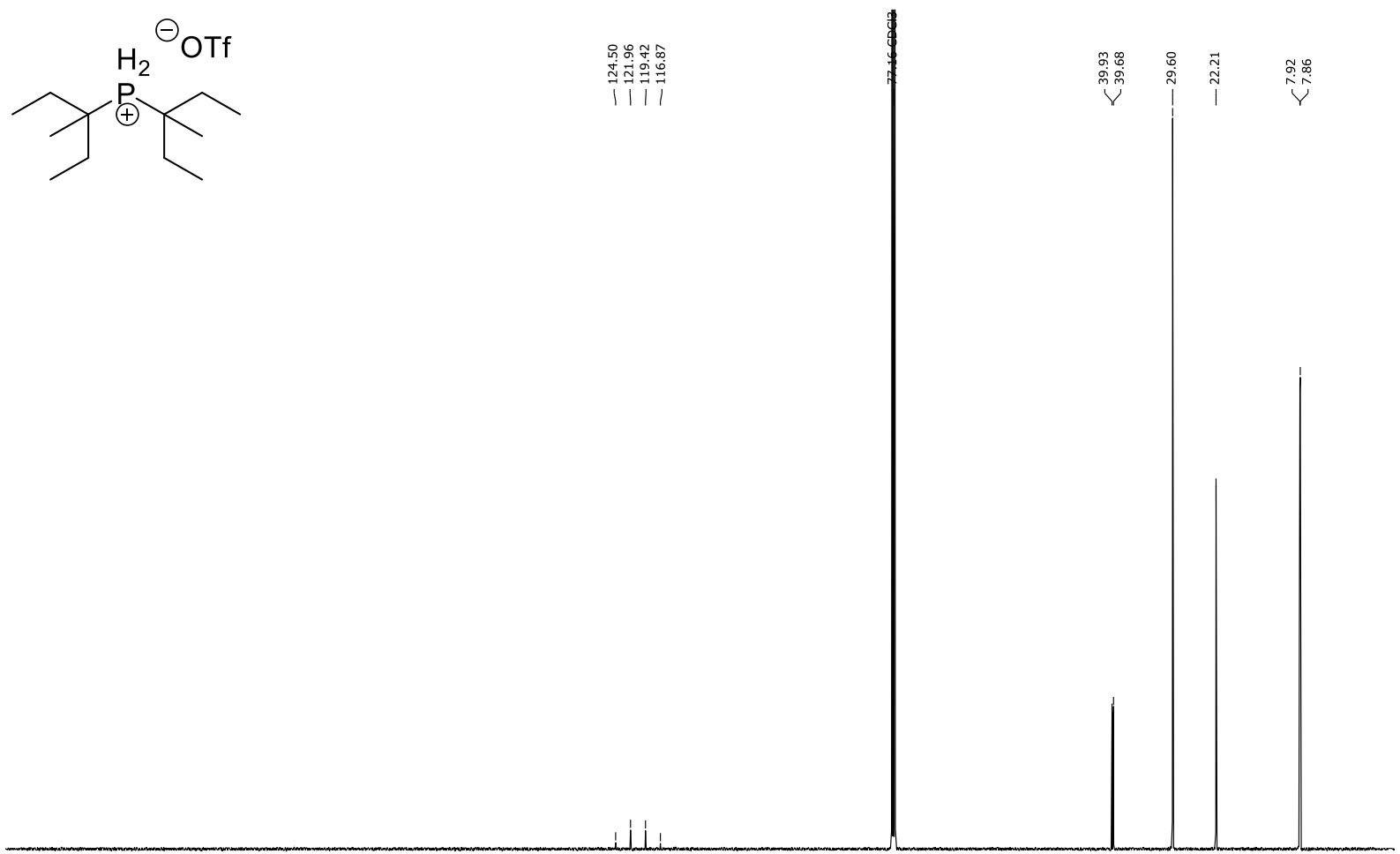

$\begin{array}{lllllllllllllllllllllll}1 & 1 \\ 220 & 210 & 200 & 190 & 180 & 170 & 160 & 150 & 140 & 130 & 120 & 110 & 100 & 90 & 80 & 70 & 60 & 50 & 40 & 30 & 20 & 10 & 0\end{array}$ 
Di-(3-methyl-3-pentyl)phosphonium triflate (1b) ${ }^{19} \mathrm{~F} \mathrm{NMR} \mathrm{(470} \mathrm{MHz}, \mathrm{CDCl}_{3}$ )<smiles>CCC(C)(CC)[Pb]C(CC)(CC)CC</smiles>

Di-(3-methyl-3-pentyl)phosphonium triflate (1b) ${ }^{31} \mathrm{P}\left\{{ }^{1} \mathrm{H}\right\}$ NMR (202 $\left.\mathrm{MHz}, \mathrm{CDCl}_{3}\right)$<smiles>CCC(C)(CC)[Pb]C(CC)(CC)CC</smiles> 
Di-(3-methyl-3-pentyl)phosphonium triflate (1b) $\left.{ }^{31} \mathrm{P} \mathrm{NMR} \mathrm{(202} \mathrm{MHz,} \mathrm{CDCl}_{3}\right)$<smiles>CCC(CC)(CC)[Pb]C(CC)(CC)CC</smiles>
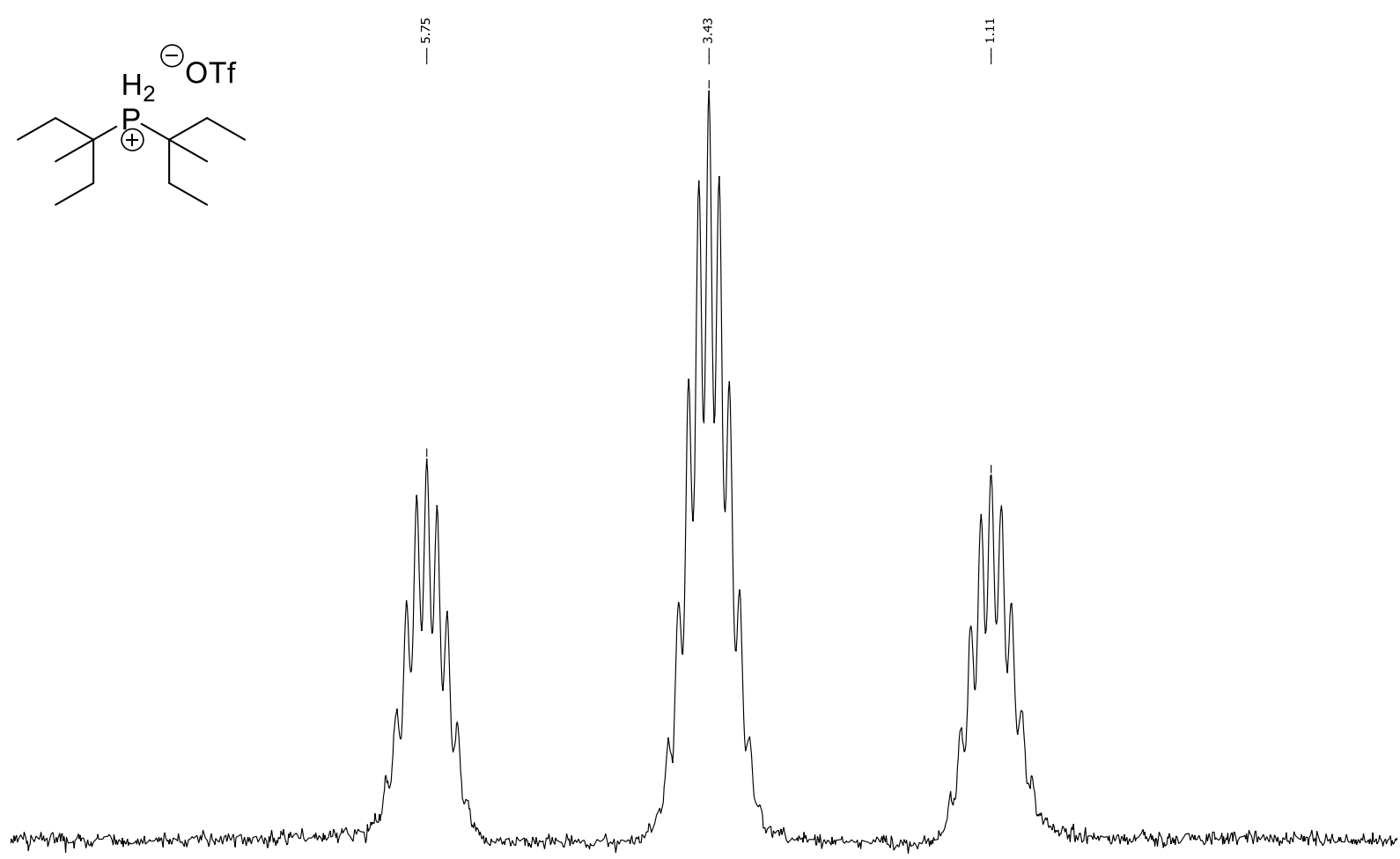

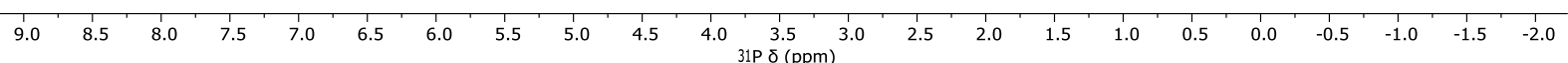


Di-(3-ethyl-3-pentyl)phosphonium triflate (1c) - ${ }^{1} \mathrm{H}$ NMR $\left(500 \mathrm{MHz}, \mathrm{CDCl}_{3}\right)$
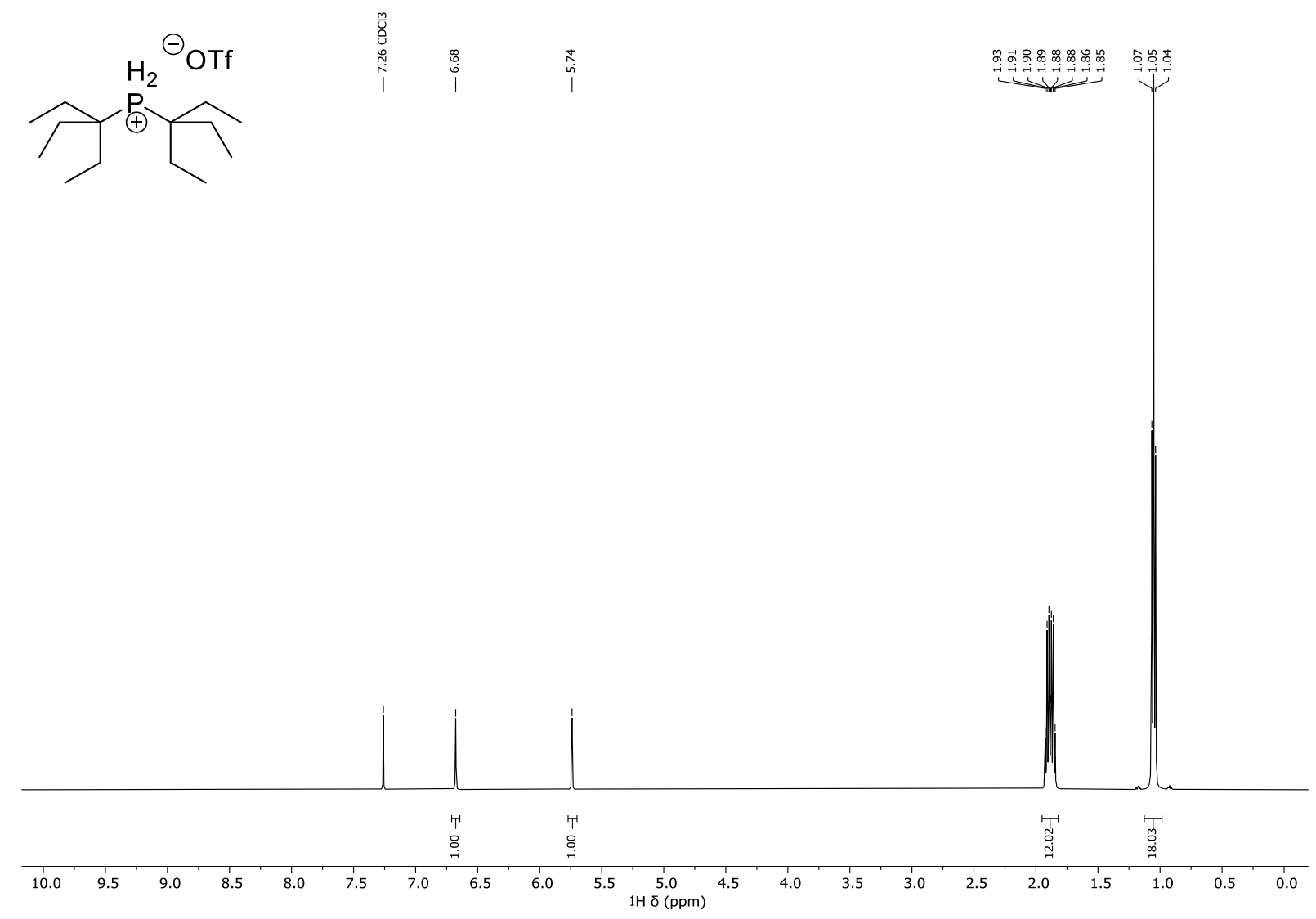

Di-(3-ethyl-3-pentyl)phosphonium triflate (1c) $-{ }^{13} \mathrm{C}\left\{{ }^{1} \mathrm{H}\right\} \mathrm{NMR}\left(126 \mathrm{MHz}, \mathrm{CDCl}_{3}\right)$

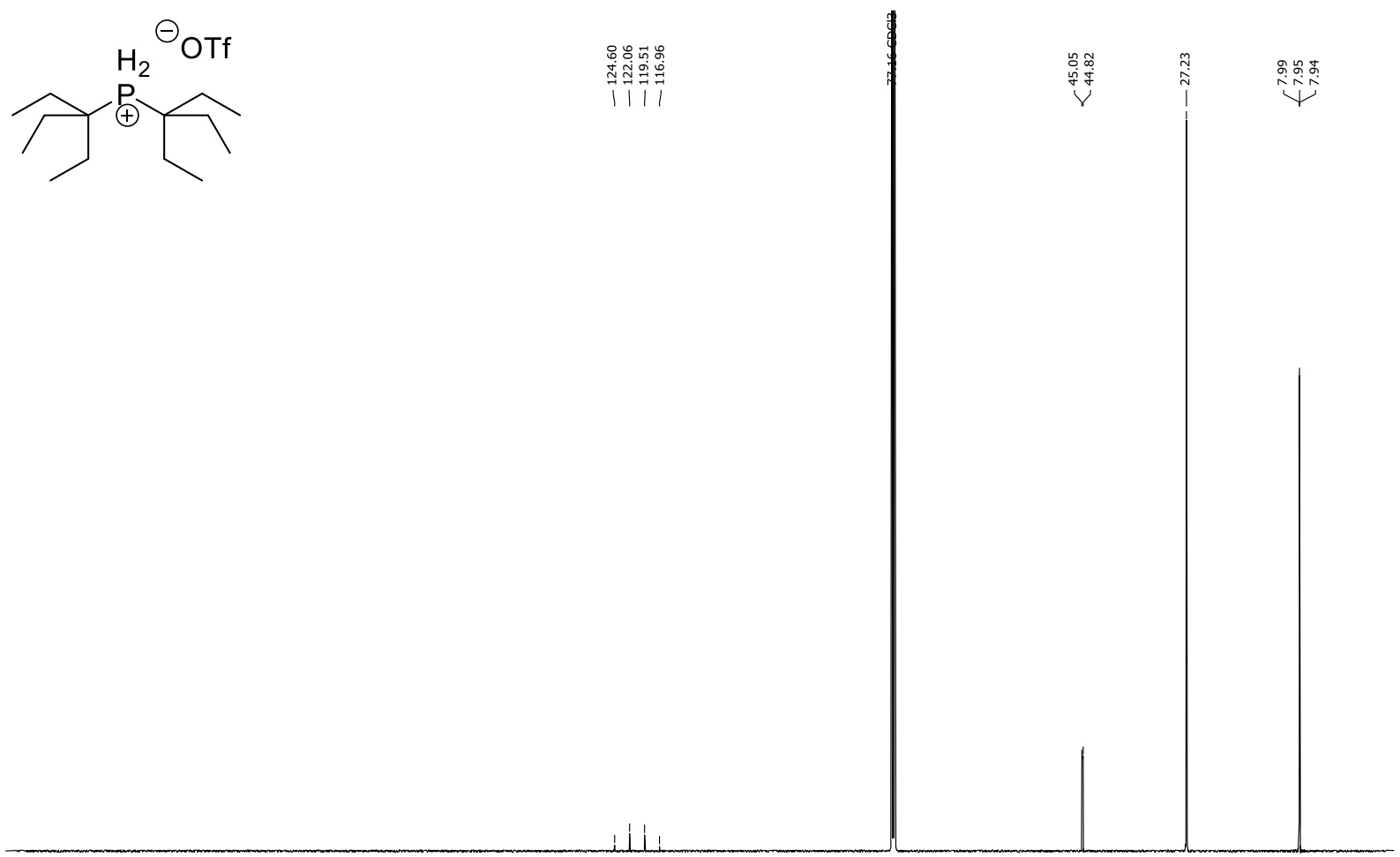

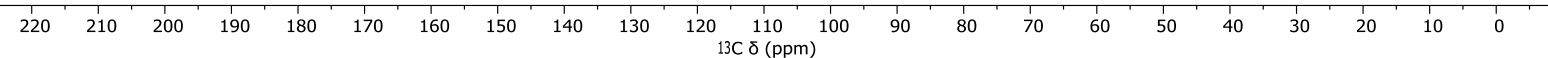


Di-(3-ethyl-3-pentyl)phosphonium triflate (1c) $\left.{ }^{19} \mathrm{~F} \mathrm{NMR} \mathrm{(470} \mathrm{MHz}, \mathrm{CDCl}_{3}\right)$<smiles>CCC(CC)(CC)[Pb]C(CC)(CC)CC</smiles>

\begin{tabular}{lllllllllllllllllllllllllll}
\hline & 10 & 10 & 0 & -10 & -20 & -30 & -40 & -50 & -60 & -70 & -80 & -90 & -100 & -110 & -120 & -130 & -140 & -150 & -160 & -170 & -180 & -190 & -200 & -210 & $-2:$
\end{tabular}

Di-(3-ethyl-3-pentyl)phosphonium triflate (1c) ${ }^{31} \mathrm{P}\left\{{ }^{1} \mathrm{H}\right\} \mathrm{NMR}\left(202 \mathrm{MHz}, \mathrm{CDCl}_{3}\right)$<smiles>CCC(CC)(CC)[Pb]C(CC)(CC)CC</smiles> 
Di-(3-ethyl-3-pentyl)phosphonium triflate (1c) ${ }^{31} \mathrm{P}$ NMR (202 MHz, $\mathrm{CDCl}_{3}$ )
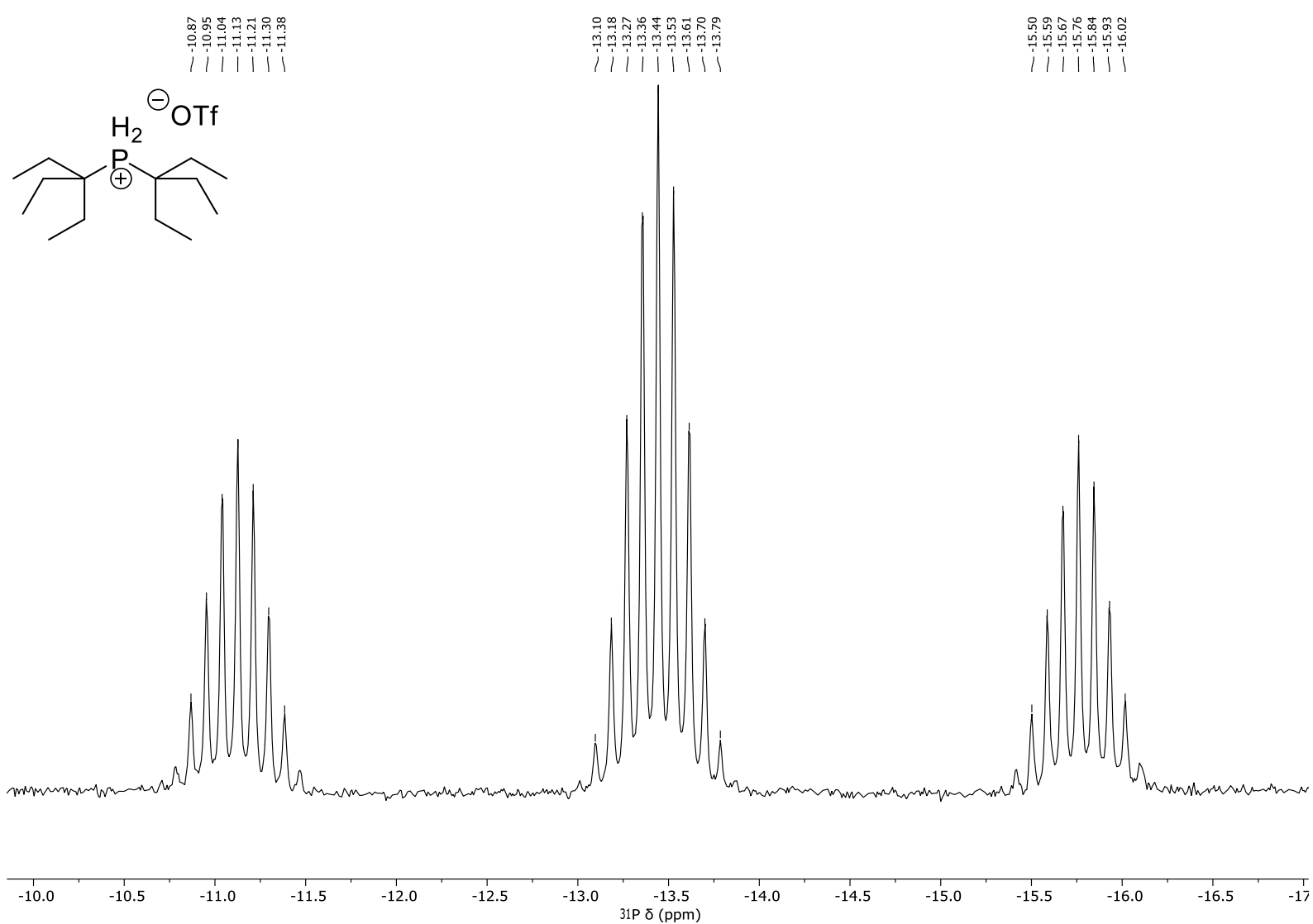
Bis-(2,3-dimethyl-2-butyl)phosphonium triflate (1d) - ${ }^{1} \mathrm{H}$ NMR $\left(500 \mathrm{MHz}, \mathrm{CDCl}_{3}\right)$
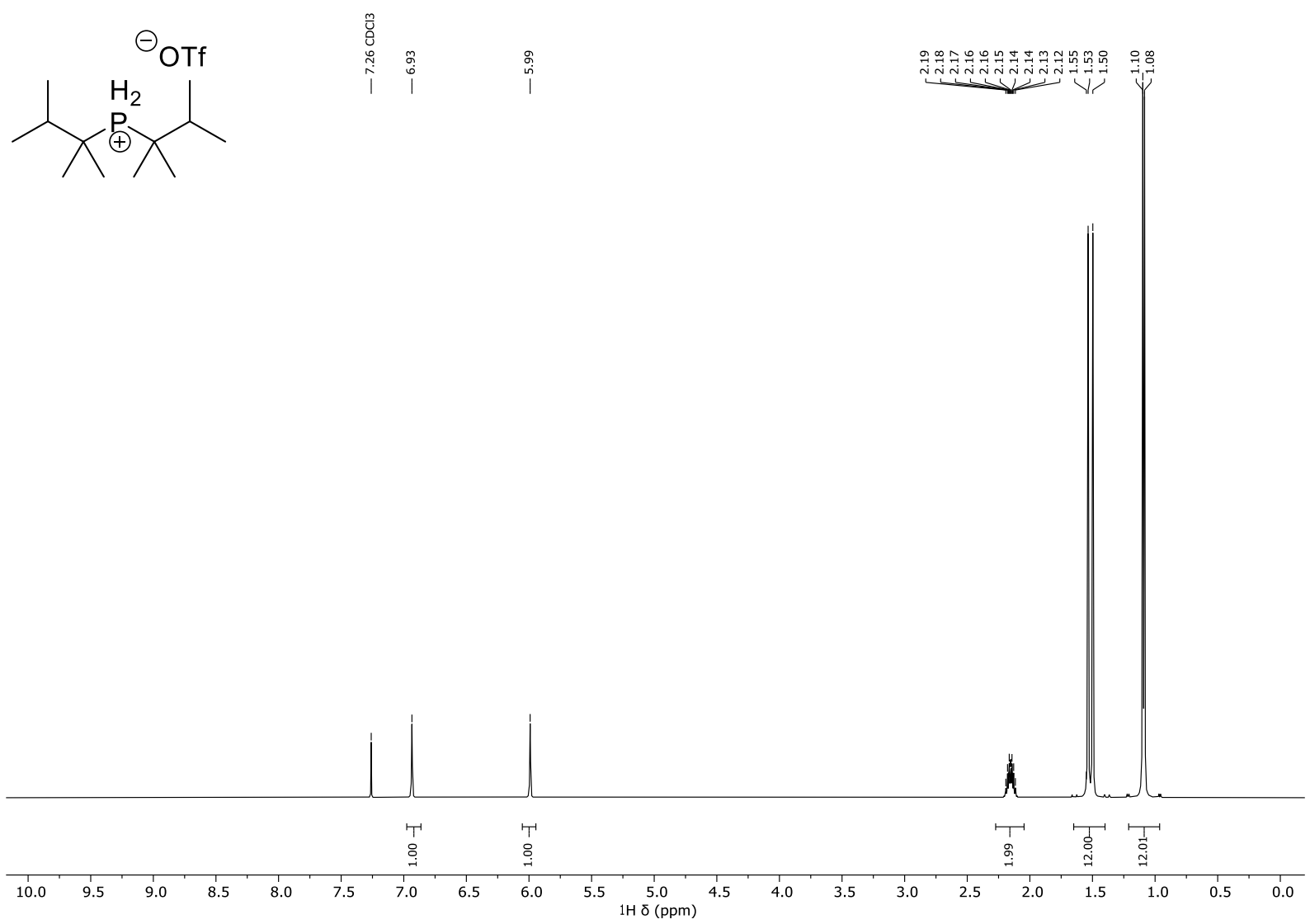

Bis-(2,3-dimethyl-2-butyl)phosphonium triflate (1d) - ${ }^{13} \mathrm{C}\left\{{ }^{1} \mathrm{H}\right\} \mathrm{NMR}\left(126 \mathrm{MHz}, \mathrm{CDCl}_{3}\right)$<smiles>CC(C)C(C)(C)PC(C)(C)C(C)[OH2+]</smiles>

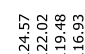


Bis-(2,3-dimethyl-2-butyl)phosphonium triflate (1d) $\left.{ }^{19} \mathrm{~F} \mathrm{NMR} \mathrm{(470} \mathrm{MHz,} \mathrm{CDCl}_{3}\right)$

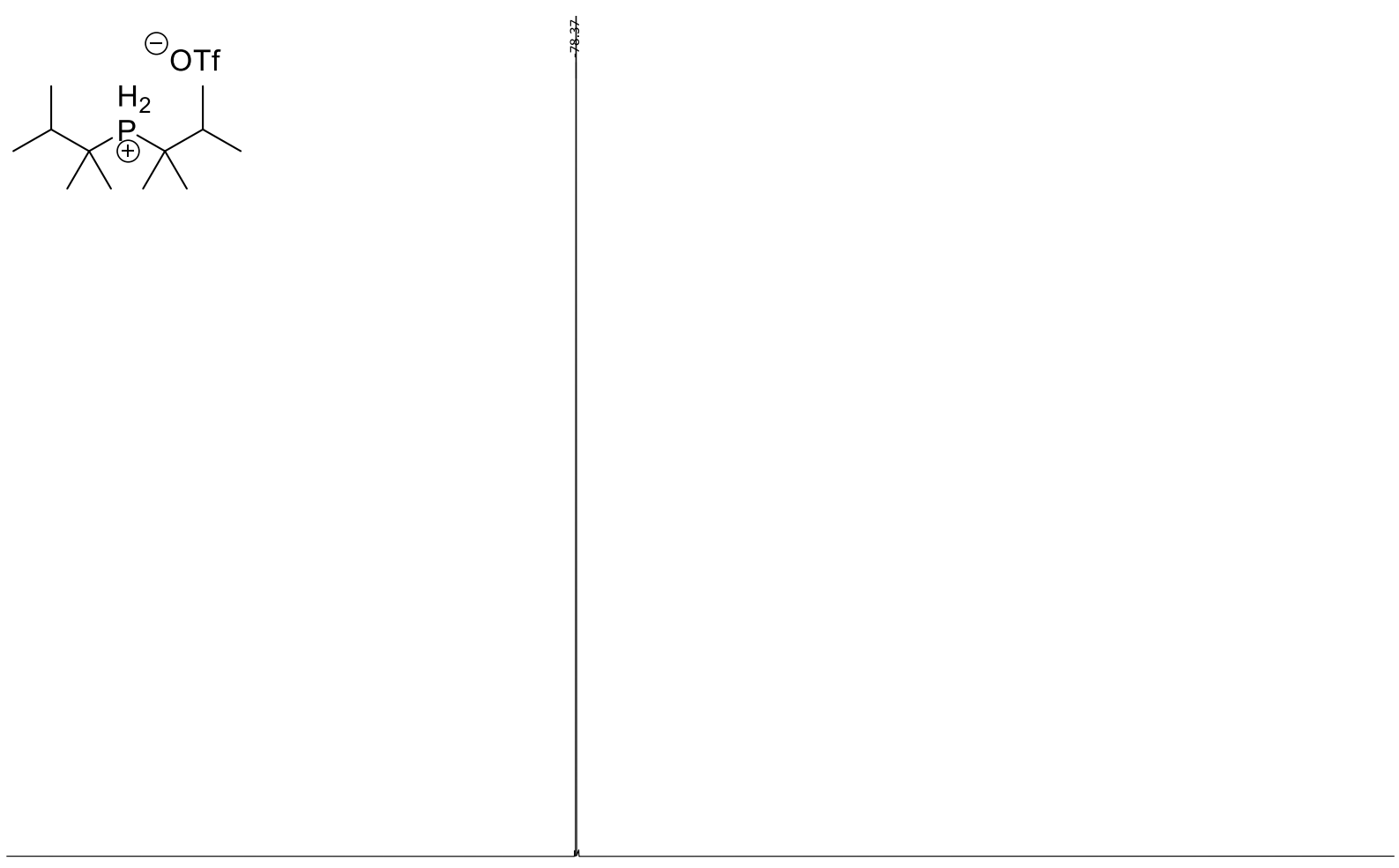

\begin{tabular}{lllllllllllllllllllllllllllll}
\hline & 10 & 10 & 0 & -10 & -20 & -30 & -40 & -50 & -60 & -70 & -80 & -90 & -100 & -110 & -120 & -130 & -140 & -150 & -160 & -170 & -180 & -190 & -200 & -210 & -2
\end{tabular}

Bis-(2,3-dimethyl-2-butyl)phosphonium triflate (1d) ${ }^{31} \mathrm{P}\left\{{ }^{1} \mathrm{H}\right\} \mathrm{NMR}\left(202 \mathrm{MHz}, \mathrm{CDCl}_{3}\right)$

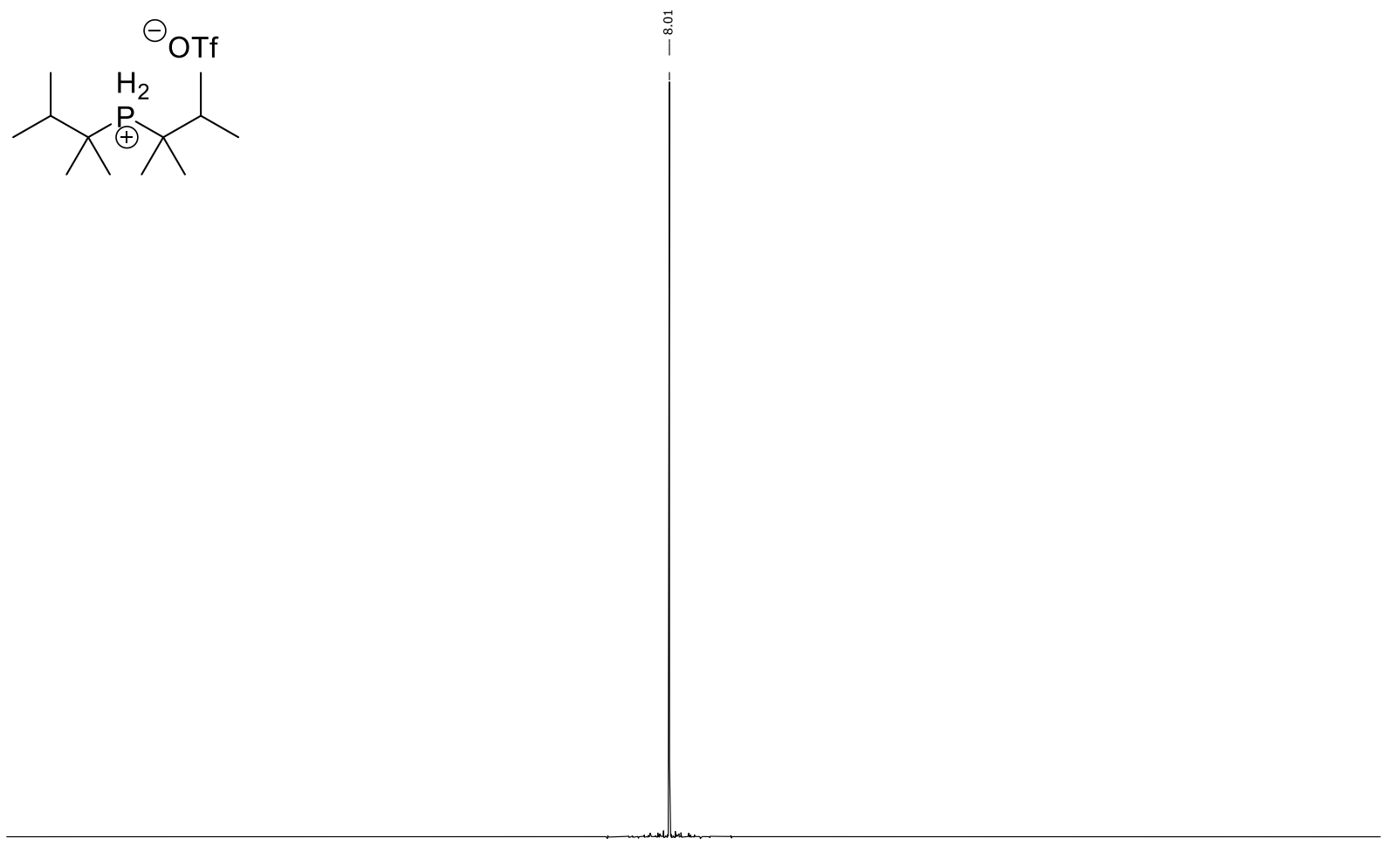

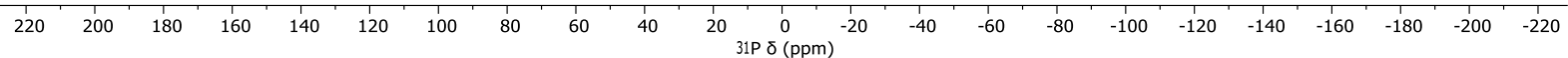


Bis-(2,3-dimethyl-2-butyl)phosphonium triflate (1d) ${ }^{31} \mathrm{P} \mathrm{NMR} \mathrm{(202} \mathrm{MHz,} \mathrm{CDCl}_{3}$ )

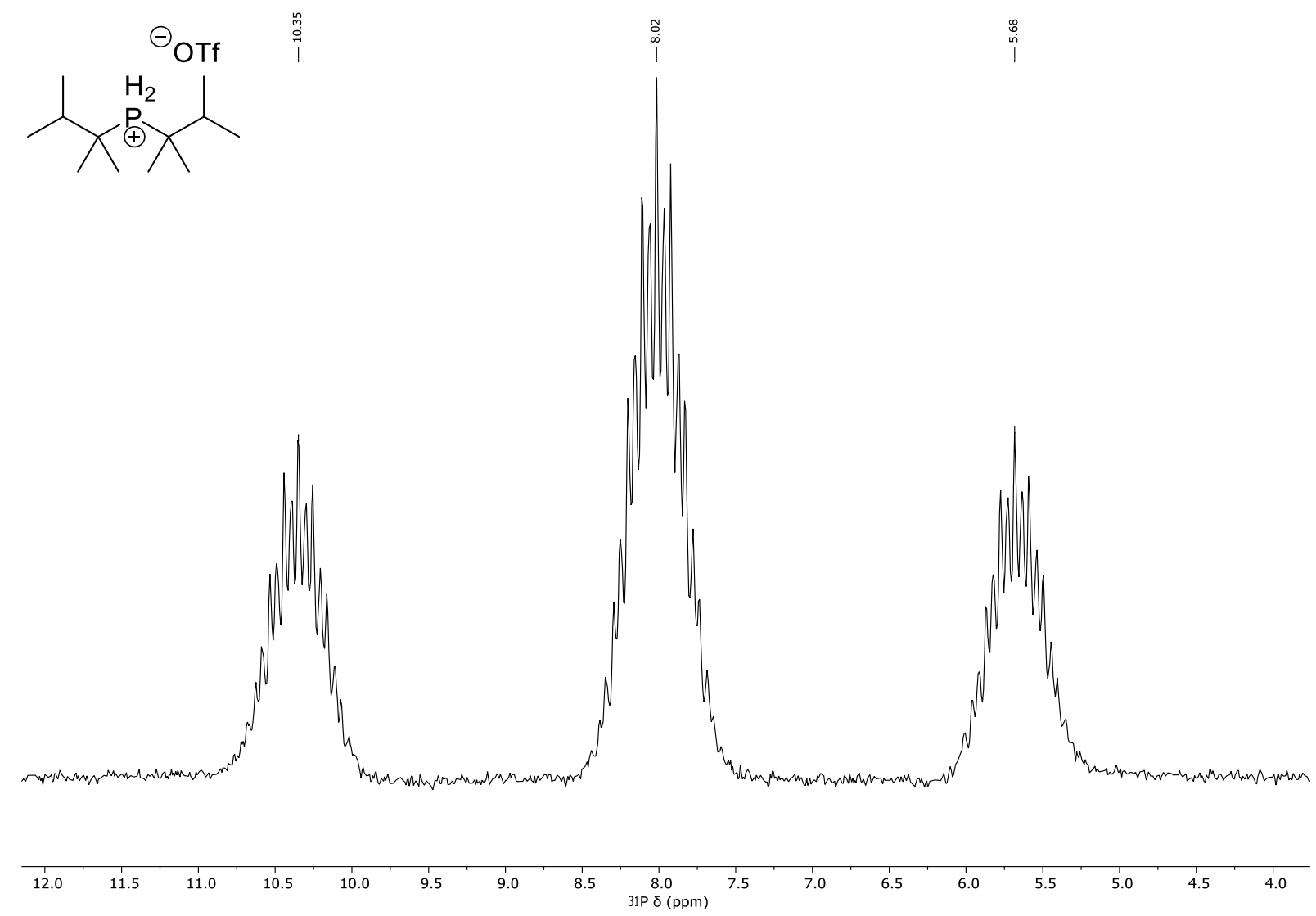


Bis-(2,3,3-trimethyl-2-butyl)phosphonium triflate (1e) - ${ }^{1} \mathrm{H}$ NMR $\left(500 \mathrm{MHz}, \mathrm{CDCl}_{3}\right)$

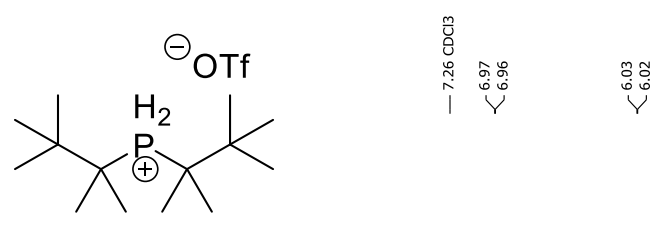

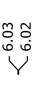

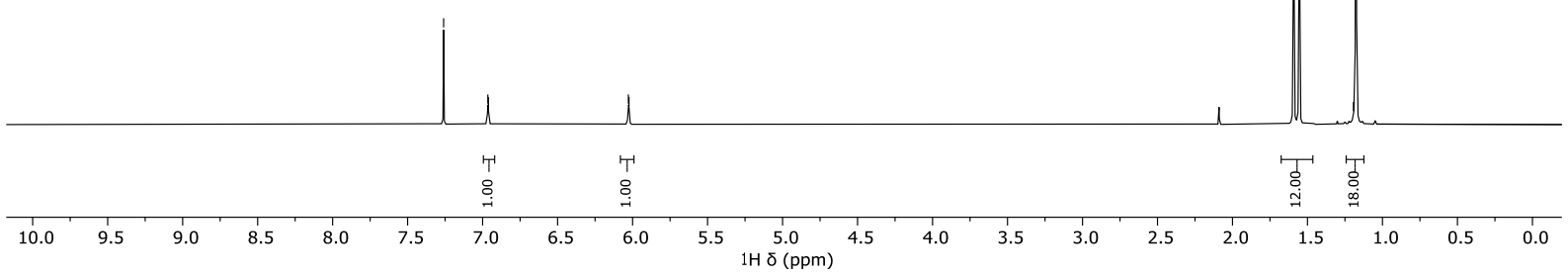

Bis-(2,3,3-trimethyl-2-butyl)phosphonium triflate $(1 \mathrm{e})-{ }^{13} \mathrm{C}\left\{{ }^{1} \mathrm{H}\right\} \mathrm{NMR}\left(126 \mathrm{MHz}, \mathrm{CDCl}_{3}\right)$

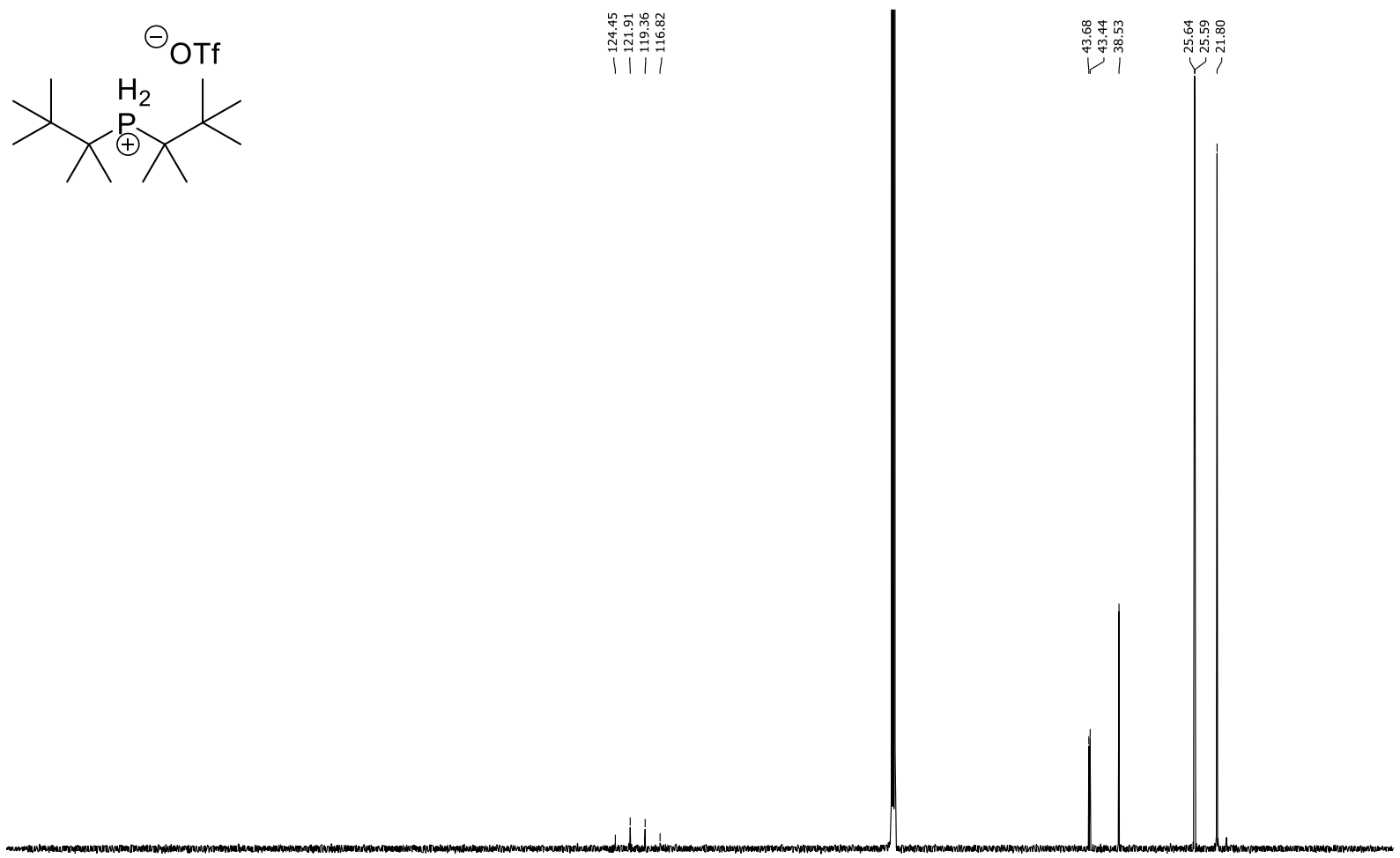

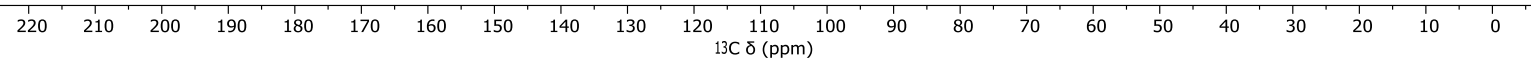


Bis-(2,3,3-trimethyl-2-butyl)phosphonium triflate (1e) ${ }^{19} \mathrm{~F} \mathrm{NMR}\left(470 \mathrm{MHz}, \mathrm{CDCl}_{3}\right)$

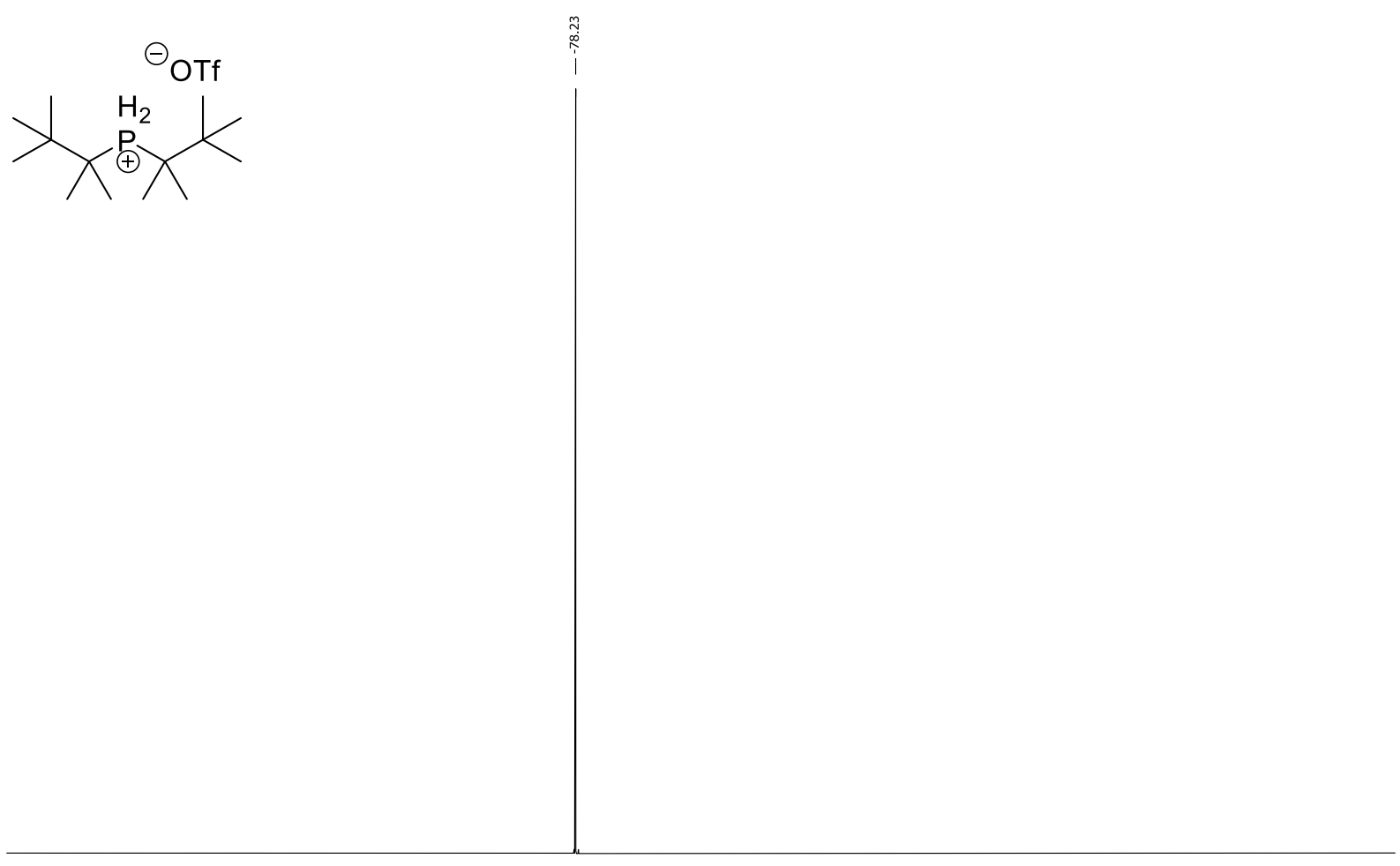

\begin{tabular}{lllllllllllllllllllllllll}
\hline 20 & 10 & 0 & -10 & -20 & -30 & -40 & -50 & -60 & -70 & -80 & -90 & -100 & -110 & -120 & -130 & -140 & -150 & -160 & -170 & -180 & -190 & -200 & -210 & $-2:$
\end{tabular}

Bis-(2,3,3-trimethyl-2-butyl)phosphonium triflate (1e) ${ }^{31} \mathrm{P}\left\{{ }^{1} \mathrm{H}\right\} \mathrm{NMR}\left(202 \mathrm{MHz}, \mathrm{CDCl}_{3}\right)$<smiles>CC(C)(C)C(C)(C)[Pb]C(C)(C)C(C)(C)[OH+]</smiles>

$\begin{array}{llllllllllllllllllllllll}220 & 200 & 180 & 160 & 140 & 120 & 100 & 80 & 60 & 40 & 20 & 0 & -20 & -40 & -60 & -80 & -100 & -120 & -140 & -160 & -180 & -200 & -220\end{array}$ 
Bis-(2,3,3-trimethyl-2-butyl)phosphonium triflate (1e) ${ }^{31} \mathrm{P} \mathrm{NMR} \mathrm{(202} \mathrm{MHz,} \mathrm{CDCl}_{3}$ )

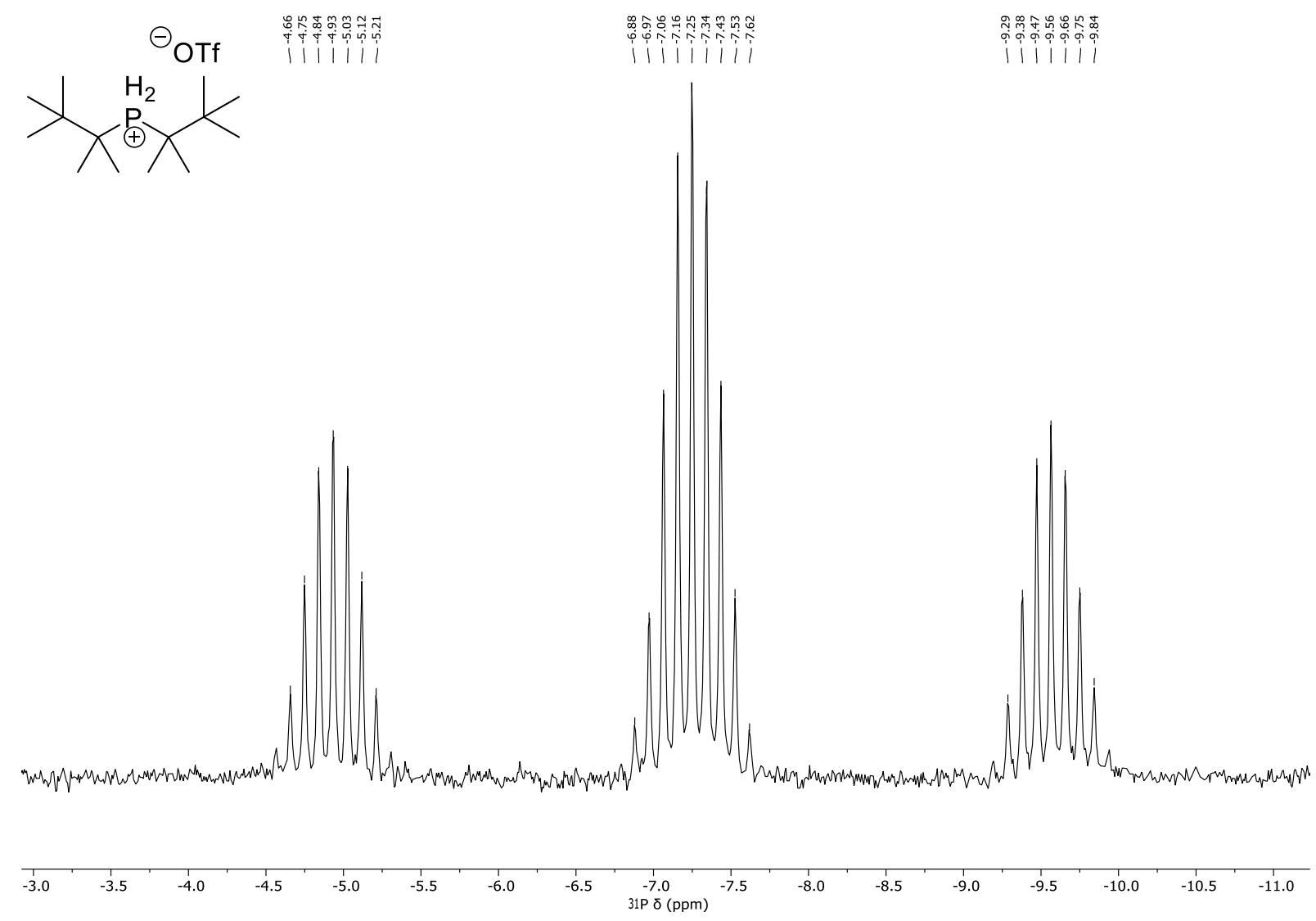


Di-(2-methyl-4-phenyl-2-butyl)phosphonium triflate (1f) - ${ }^{1} \mathrm{H}$ NMR (500 $\left.\mathrm{MHz}, \mathrm{CDCl}_{3}\right)$

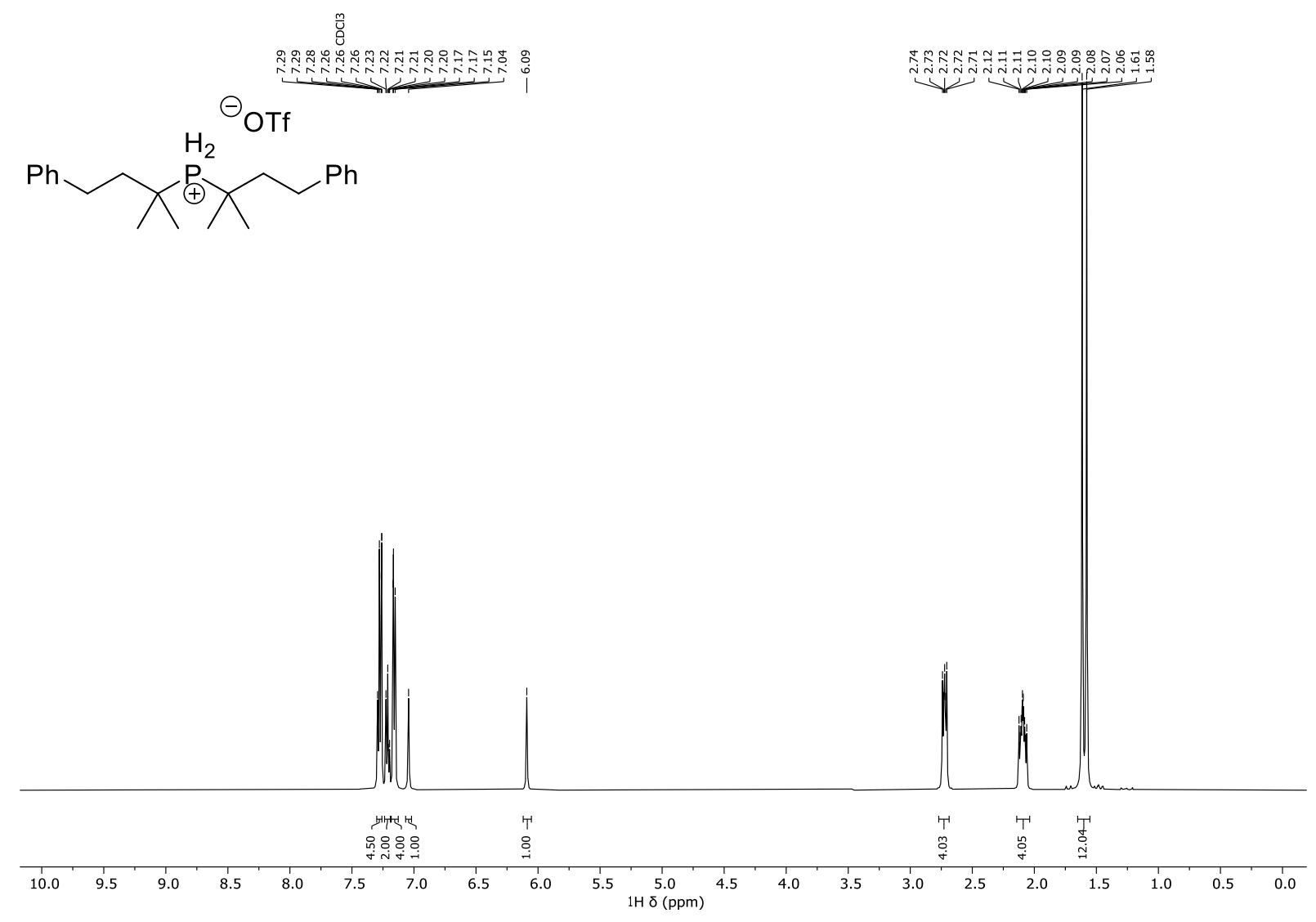

Di-(2-methyl-4-phenyl-2-butyl)phosphonium triflate (1f) - ${ }^{13} \mathrm{C}\left\{{ }^{1} \mathrm{H}\right\} \mathrm{NMR}\left(126 \mathrm{MHz}, \mathrm{CDCl}_{3}\right)$

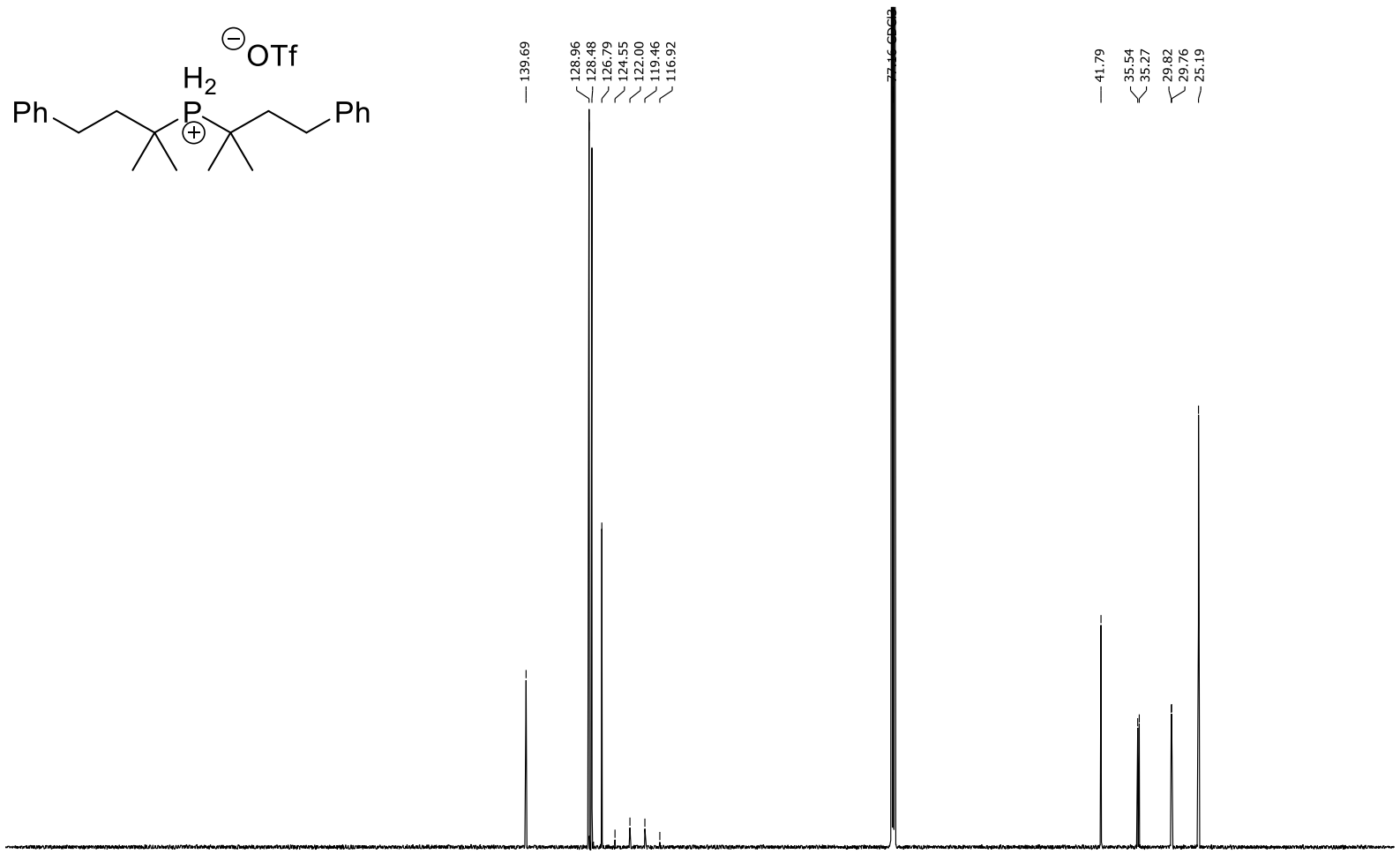

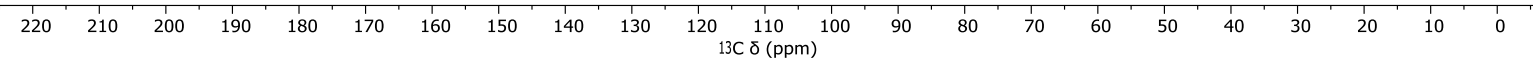


Di-(2-methyl-4-phenyl-2-butyl)phosphonium triflate (1f) $\left.{ }^{19} \mathrm{~F} \mathrm{NMR} \mathrm{(470} \mathrm{MHz}, \mathrm{CDCl}_{3}\right)$

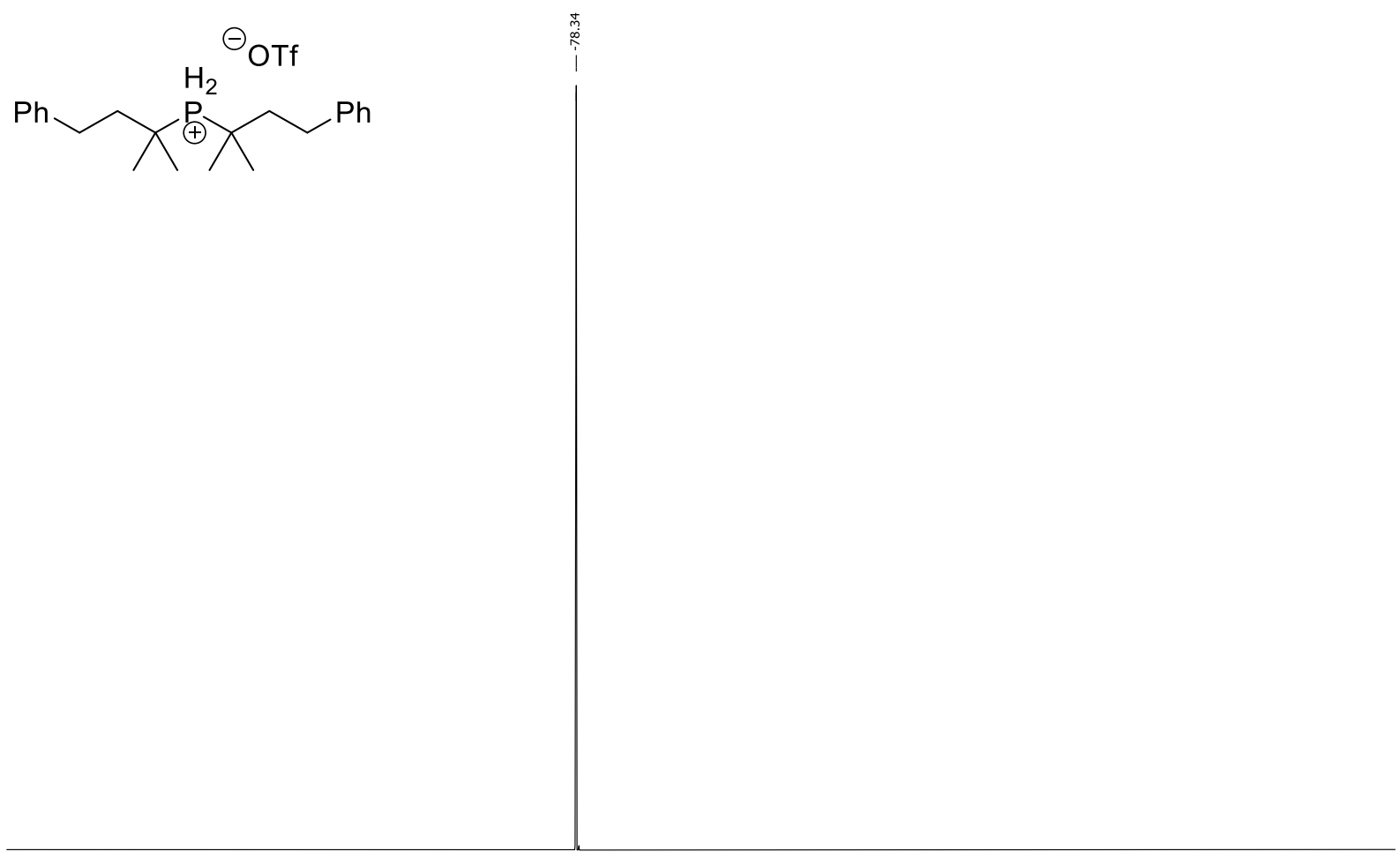

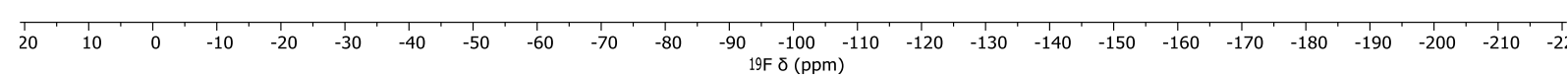

Di-(2-methyl-4-phenyl-2-butyl)phosphonium triflate (1f) ${ }^{31} \mathrm{P}\left\{{ }^{1} \mathrm{H}\right\} \mathrm{NMR}\left(202 \mathrm{MHz}, \mathrm{CDCl}_{3}\right)$<smiles>CC(C)([B]C(C)(C)CCc1ccccc1)CCc1ccccc1</smiles>

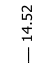

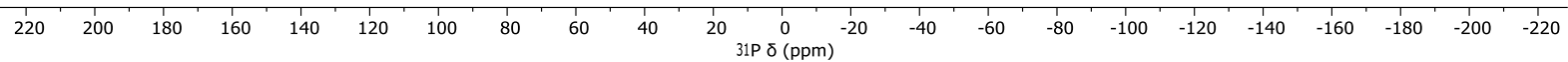


Di-(2-methyl-4-phenyl-2-butyl)phosphonium triflate (1f) ${ }^{31} \mathrm{P}$ NMR (202 MHz, $\mathrm{CDCl}_{3}$ )

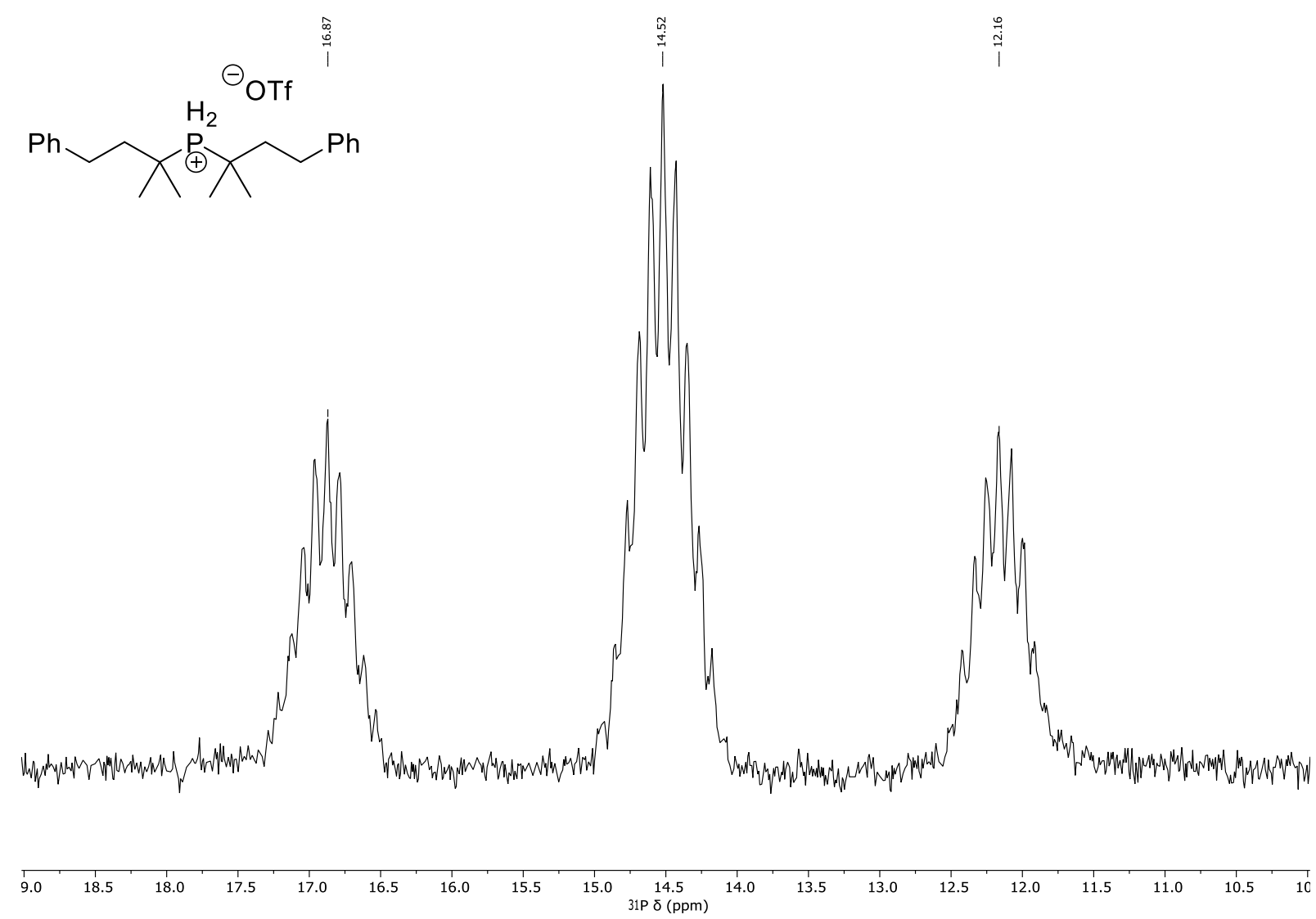


Di-(2-Methyl-4-(4-bromophenyl)-2-butyl)phosphonium triflate (1g) - ${ }^{1} \mathrm{H}$ NMR (500 $\left.\mathrm{MHz} \mathrm{CDCl}_{3}\right)$<smiles>CC(C)(CCc1ccc(Br)cc1)PC(C)(C)CCc1ccc(Br)cc1</smiles>

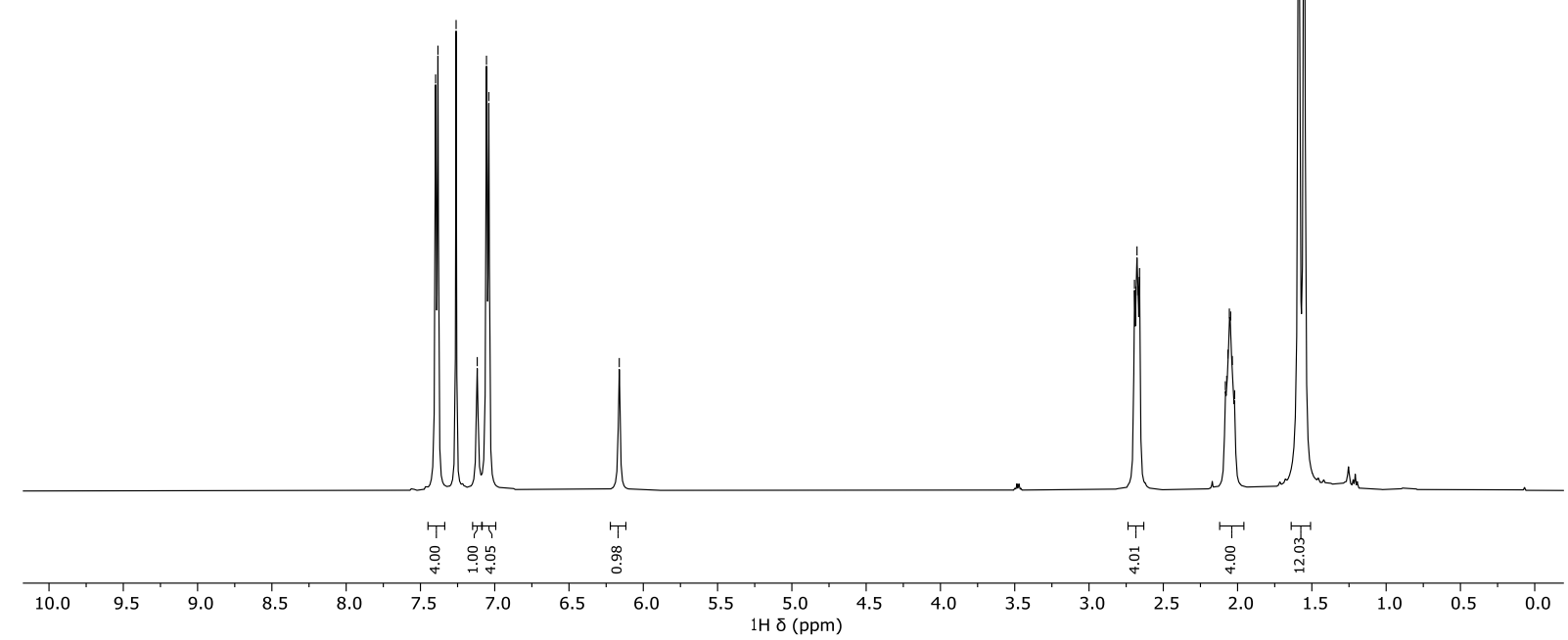

Di-(2-Methyl-4-(4-bromophenyl)-2-butyl)phosphonium triflate (1g) - ${ }^{13} \mathrm{C}\left\{{ }^{1} \mathrm{H}\right\} \mathrm{NMR}(126 \mathrm{MHz}, \mathrm{CDCl})$

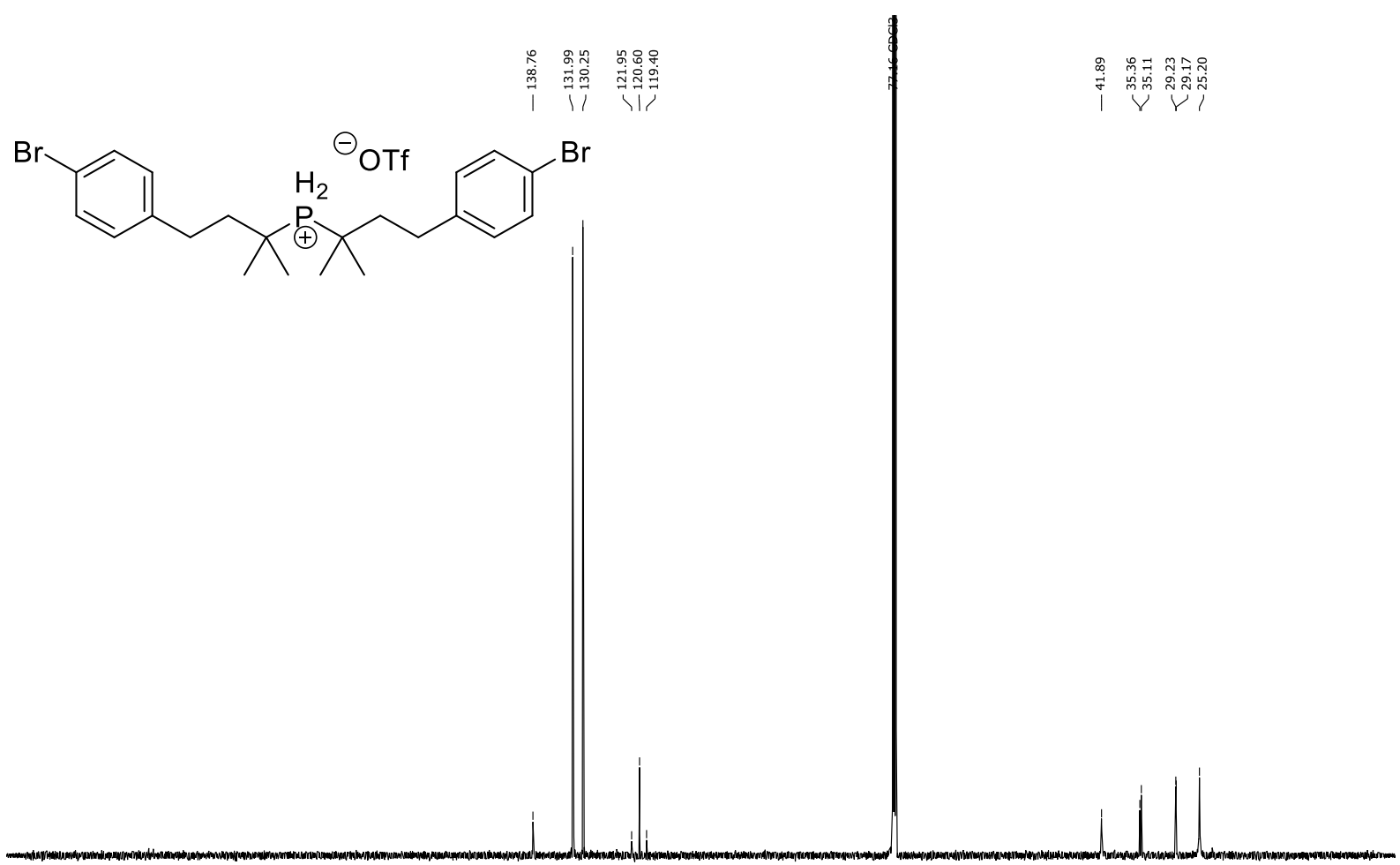

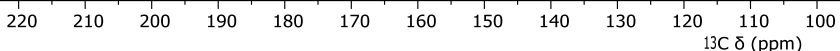


Di-(2-Methyl-4-(4-bromophenyl)-2-butyl)phosphonium triflate (1g) ${ }^{19} \mathrm{~F}$ NMR (470 $\left.\mathrm{MHz}, \mathrm{CDCl}_{3}\right)$
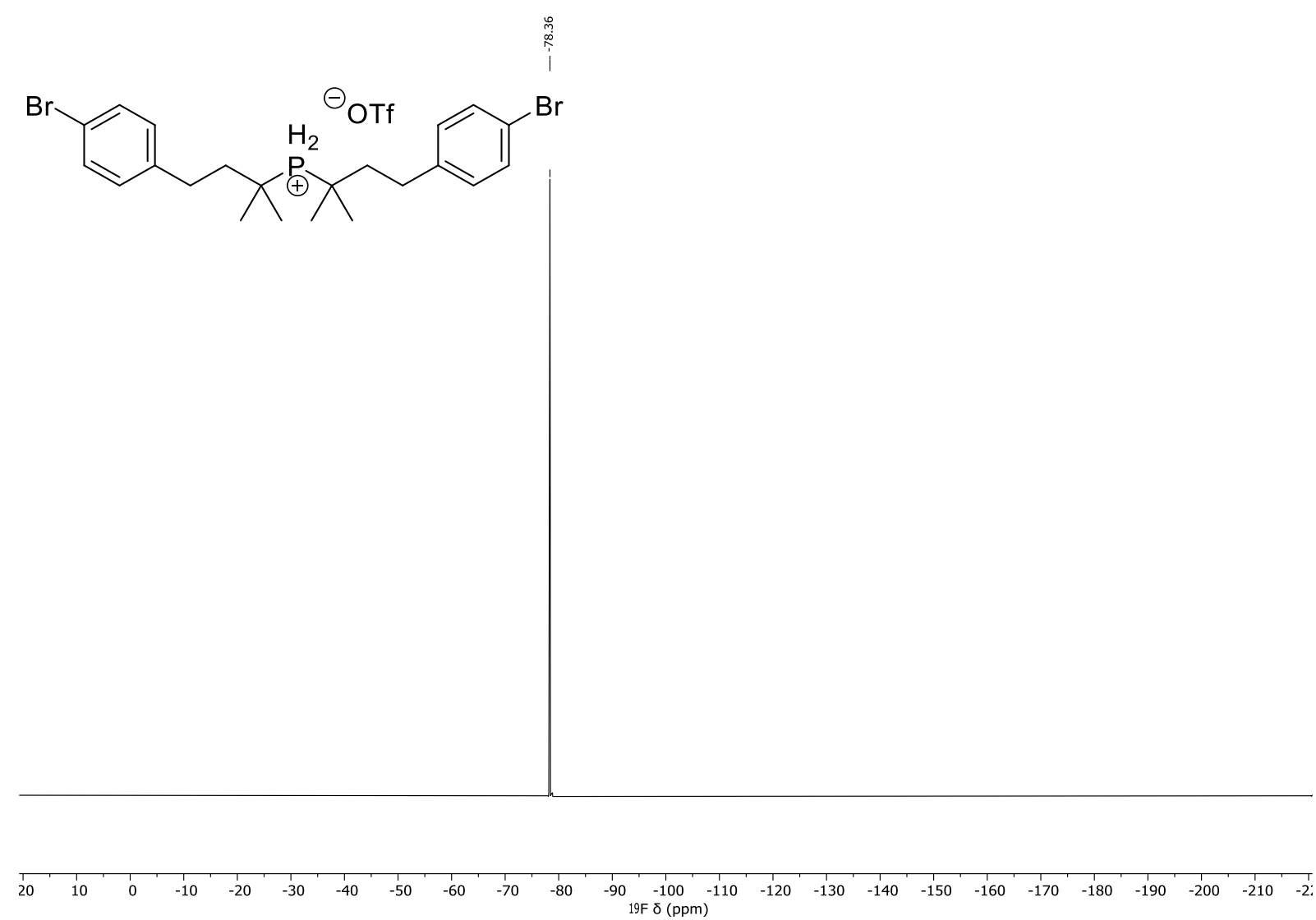

Di-(2-Methyl-4-(4-bromophenyl)-2-butyl)phosphonium triflate $(1 \mathrm{~g}){ }^{31} \mathrm{P}\left\{{ }^{1} \mathrm{H}\right\} \mathrm{NMR}\left(202 \mathrm{MHz}, \mathrm{CDCl}_{3}\right)$<smiles>CC(C)(CCc1ccc(Br)cc1)PC(C)(C)CCc1ccc(Br)cc1</smiles> 
Di-(2-Methyl-4-(4-bromophenyl)-2-butyl)phosphonium triflate (1g) ${ }^{31} \mathrm{P}$ NMR (202 $\mathrm{MHz}, \mathrm{CDCl}_{3}$ )
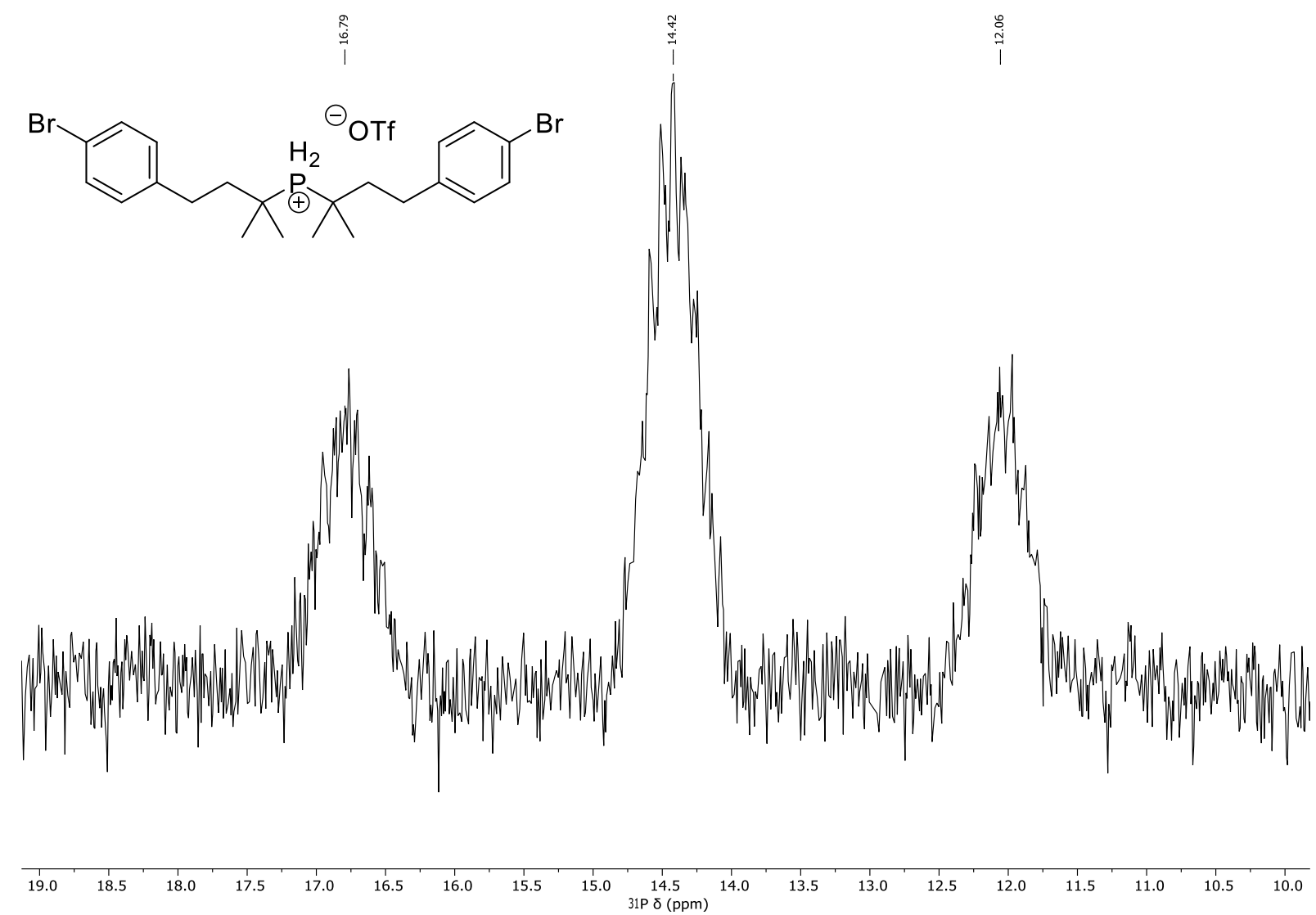
Di-(1-methylcyclopentyl)phosphonium triflate (1h) - ${ }^{1} \mathrm{H}$ NMR $\left(500 \mathrm{MHz}, \mathrm{CDCl}_{3}\right)$


Di-(1-methylcyclopentyl)phosphonium triflate (1h) $-{ }^{13} \mathrm{C}\left\{{ }^{1} \mathrm{H}\right\} \mathrm{NMR}\left(126 \mathrm{MHz}, \mathrm{CDCl}_{3}\right)$<smiles>CC1(PC2(C)CCCC2)CCCC1</smiles>

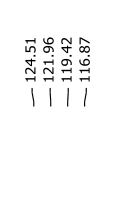

$\begin{array}{llllllllllll}220 & 210 & 200 & 190 & 180 & 170 & 160 & 150 & 140 & 130 & 120 & 110 \\ 13 \mathrm{C} \delta(\mathrm{ppm})\end{array}$ 
Di-(1-methylcyclopentyl)phosphonium triflate $\left.(1 \mathrm{~h}){ }^{19} \mathrm{~F} \mathrm{NMR} \mathrm{(470} \mathrm{MHz}, \mathrm{CDCl}_{3}\right)$
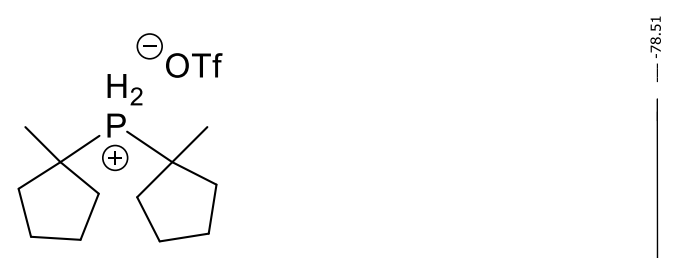

\begin{tabular}{lllllllllllllllllllllllllll}
\hline 20 & 10 & 0 & -10 & -20 & -30 & -40 & -50 & -60 & -70 & -80 & -90 & -100 & -110 & -120 & -130 & -140 & -150 & -160 & -170 & -180 & -190 & -200 & -210 & $-2:$
\end{tabular}

Di-(1-methylcyclopentyl)phosphonium triflate $(1 \mathrm{~h}){ }^{31} \mathrm{P}\left\{{ }^{1} \mathrm{H}\right\} \mathrm{NMR}\left(202 \mathrm{MHz}, \mathrm{CDCl}_{3}\right)$

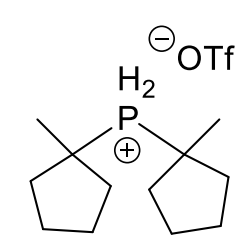

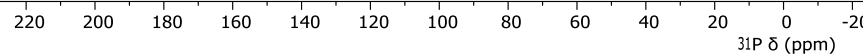


Di-(1-methylcyclopentyl)phosphonium triflate (1h) ${ }^{31}$ P NMR (202 MHz, $\mathrm{CDCl}_{3}$ )

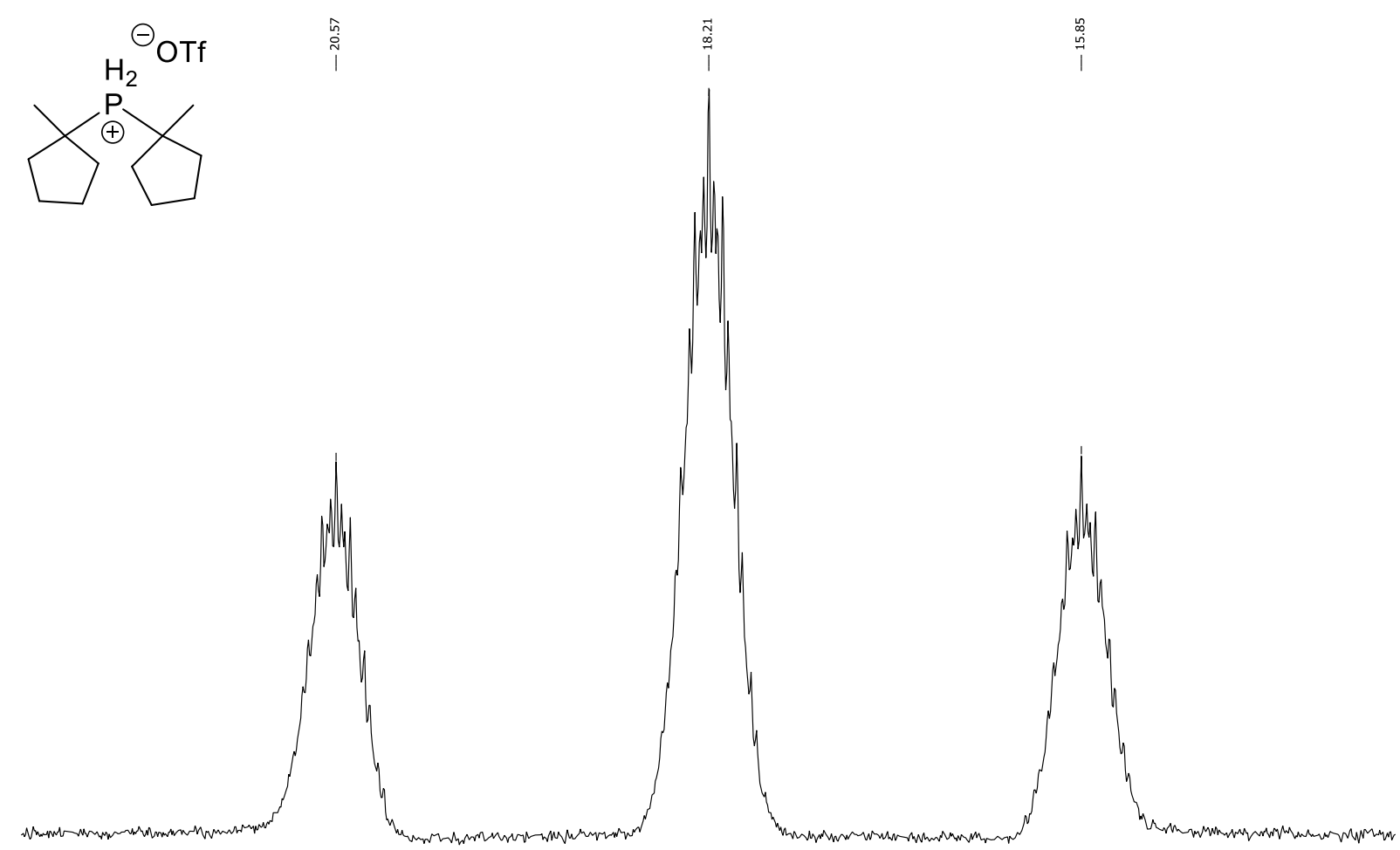

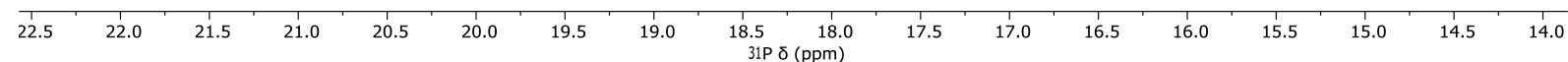


Di-(1-methylcyclohexyl)phosphonium triflate (1i) - ${ }^{1} \mathrm{H}$ NMR $\left(500 \mathrm{MHz}, \mathrm{CDCl}_{3}\right)$

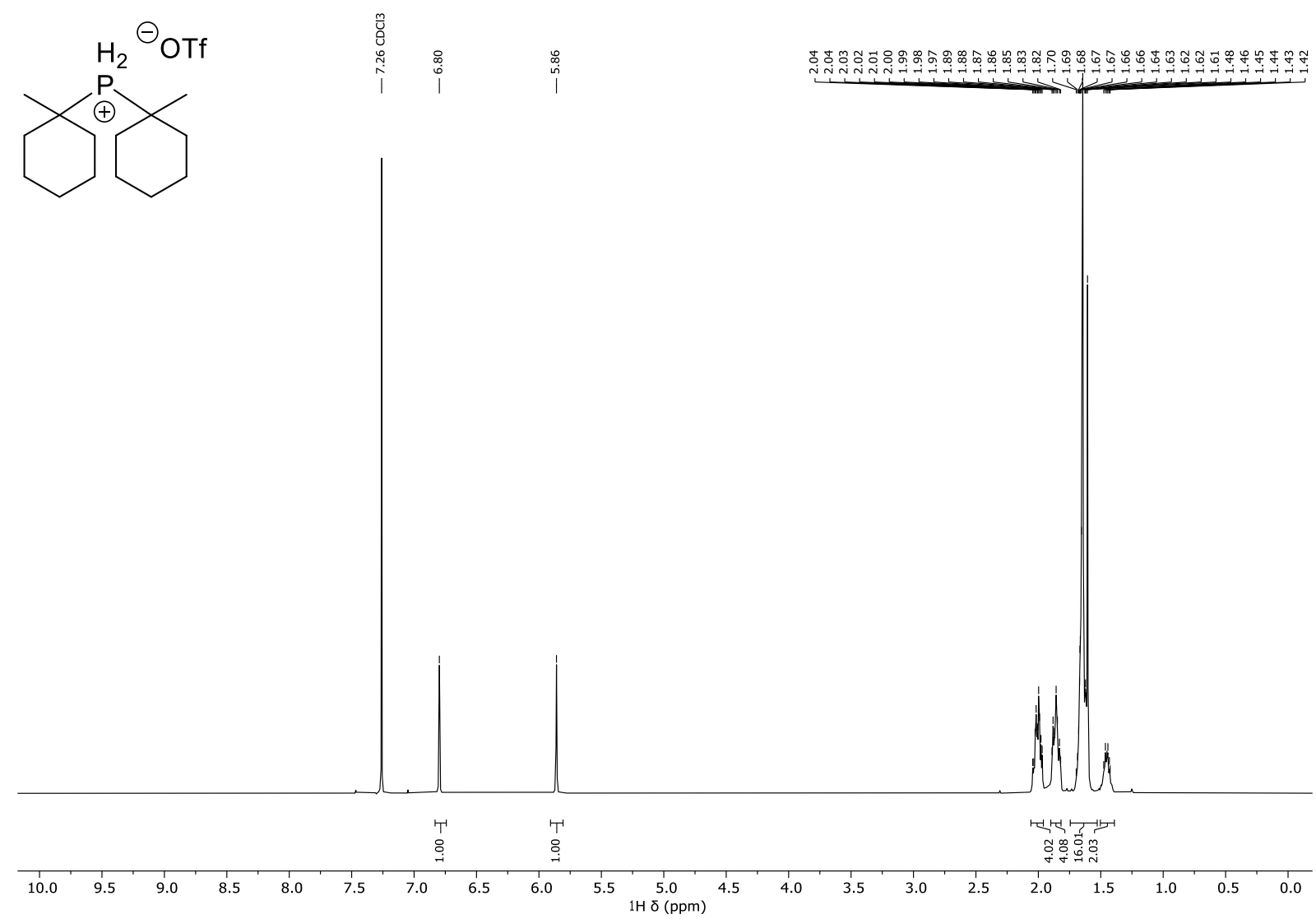

Di-(1-methylcyclohexyl)phosphonium triflate $(1 \mathrm{i})-{ }^{13} \mathrm{C}\left\{{ }^{1} \mathrm{H}\right\}$ NMR $\left(126 \mathrm{MHz}, \mathrm{CDCl}_{3}\right)$<smiles>CC12CCCCC(CCC1)C2(C)[OH2+]</smiles>

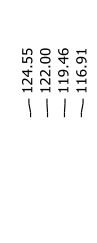


Di-(1-methylcyclohexyl)phosphonium triflate (1i) ${ }^{19} \mathrm{~F} \mathrm{NMR}\left(470 \mathrm{MHz}, \mathrm{CDCl}_{3}\right)$<smiles>CC1([OH2+])PC2CCCCC21C</smiles>

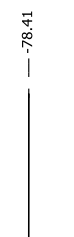

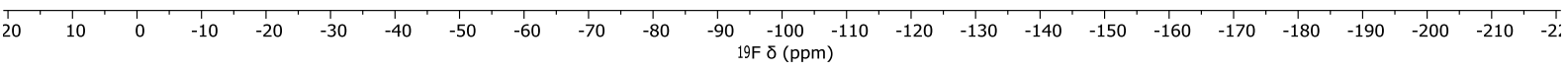

Di-(1-methylcyclohexyl)phosphonium triflate (1i) ${ }^{31} \mathrm{P}\left\{{ }^{1} \mathrm{H}\right\} \mathrm{NMR}\left(202 \mathrm{MHz}, \mathrm{CDCl}_{3}\right)$<smiles>CC1([OH2+])PC2CCCCC21C</smiles> 
Di-(1-methylcyclohexyl)phosphonium triflate (1i) ${ }^{31} \mathrm{P}$ NMR (202 MHz, $\mathrm{CDCl}_{3}$ )<smiles></smiles>

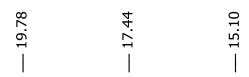

P

$\checkmark$
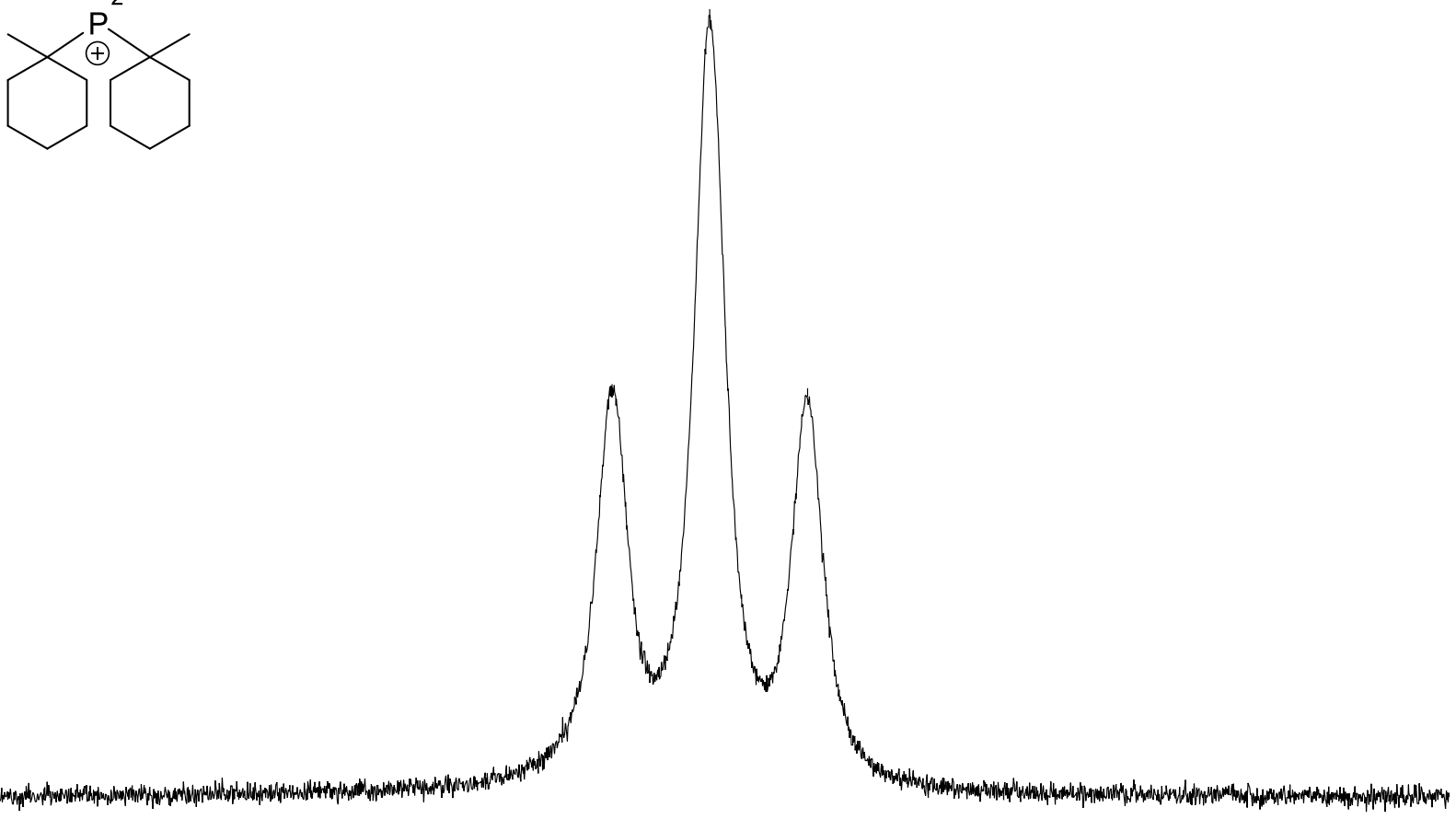

\begin{tabular}{lllllllllllllllllllllllllllllllllllllllllllllllllll}
\hline 34 & 33 & 32 & 31 & 30 & 29 & 28 & 27 & 26 & 25 & 24 & 23 & 22 & 21 & 20 & 19 & 18 & 17 & 16 & 15 & 14 & 13 & 12 & 11 & 10 & 9 & 8 & 7 & 6 & 5 & 4 & 3 & 2 & 1 & 0
\end{tabular} 
Di-(1-phenylcyclohexyl)phosphonium triflate (1j) - ${ }^{1} \mathrm{H} \mathrm{NMR}\left(500 \mathrm{MHz}, \mathrm{CDCl}_{3}\right)$

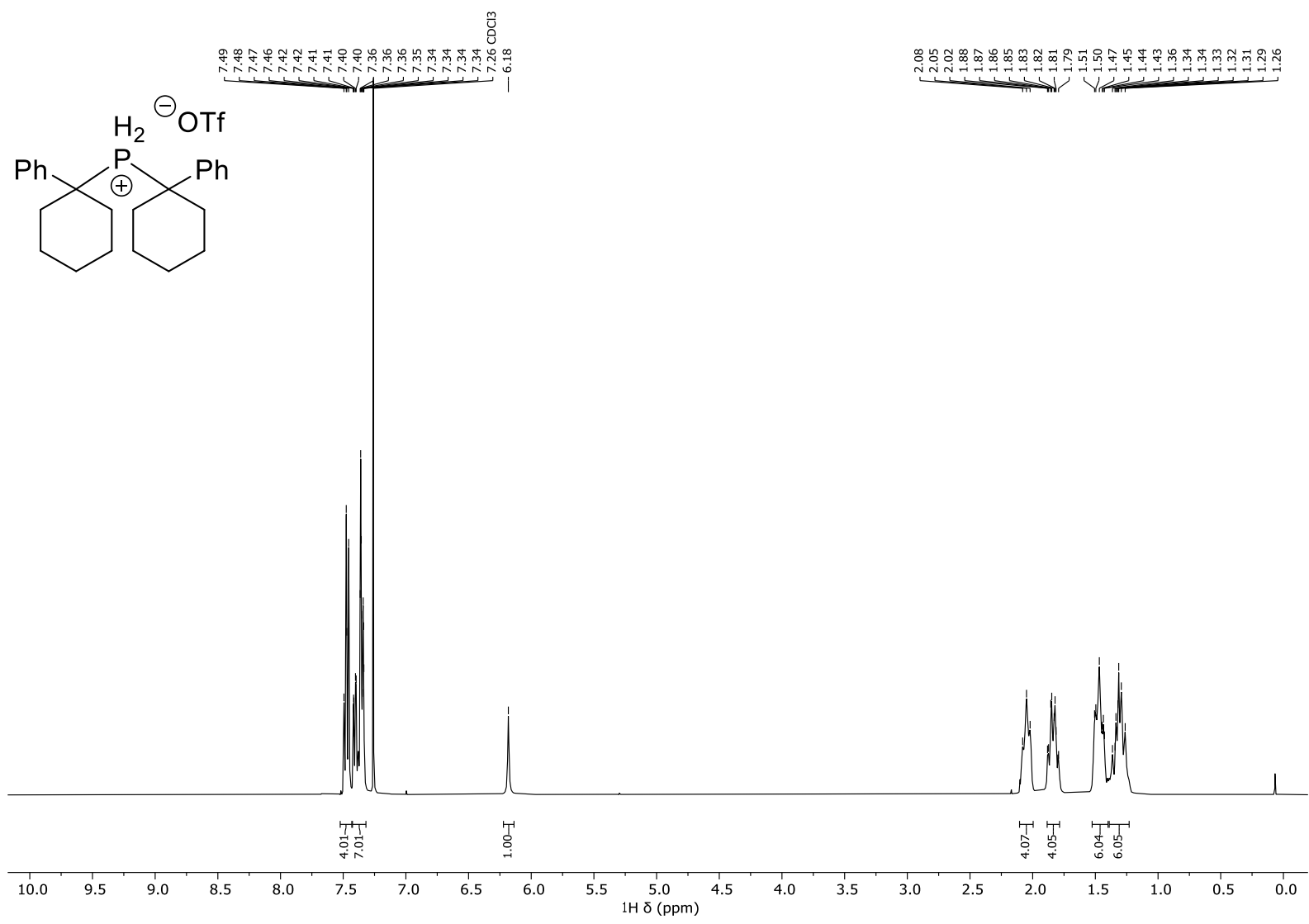

Di-(1-phenylcyclohexyl)phosphonium triflate $(1 \mathrm{j})-{ }^{13} \mathrm{C}\left\{{ }^{1} \mathrm{H}\right\}$ NMR $\left(126 \mathrm{MHz}, \mathrm{CDCl}_{3}\right)$

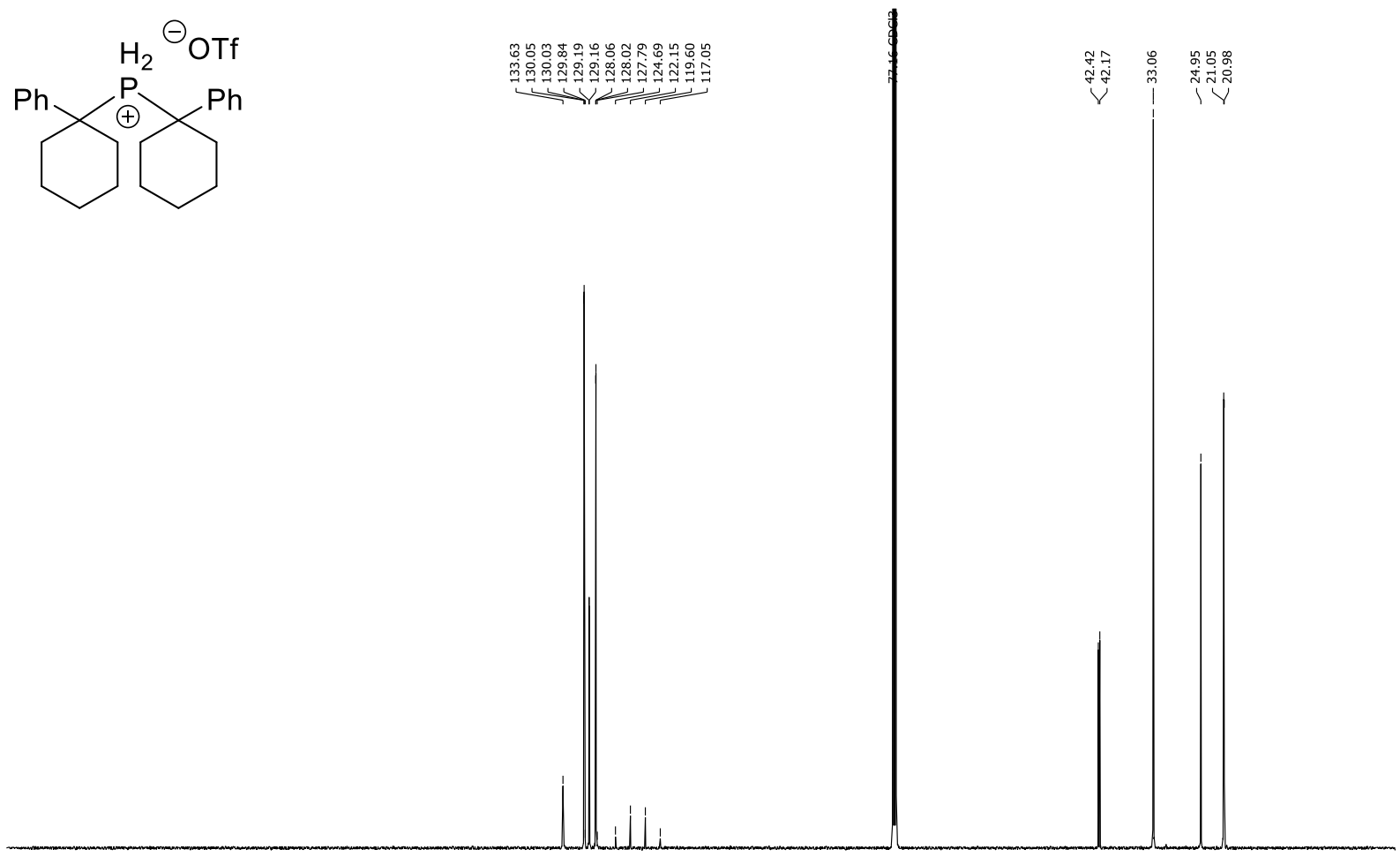

$\begin{array}{llllllllllllllllllllllllll}220 & 210 & 200 & 190 & 180 & 170 & 160 & 150 & 140 & 130 & 120 & 110 & 100 & 90 & 80 & 70 & 60 & 50 & 40 & 30 & 20 & 10 & 0\end{array}$ 
Di-(1-phenylcyclohexyl)phosphonium triflate $(1 \mathrm{j}){ }^{19} \mathrm{~F} \mathrm{NMR}\left(470 \mathrm{MHz}, \mathrm{CDCl}_{3}\right)$<smiles>[P-]PC1(c2ccccc2)CCCCC1</smiles>

Di-(1-phenylcyclohexyl)phosphonium triflate $(1 \mathrm{j}){ }^{31} \mathrm{P}\left\{{ }^{1} \mathrm{H}\right\} \mathrm{NMR}\left(202 \mathrm{MHz}, \mathrm{CDCl}_{3}\right)$<smiles>[P-]PC1(c2ccccc2)CCCCC1</smiles> 
Di-(1-phenylcyclohexyl)phosphonium triflate (1j) ${ }^{31}$ P NMR (202 MHz, $\left.\mathrm{CDCl}_{3}\right)$
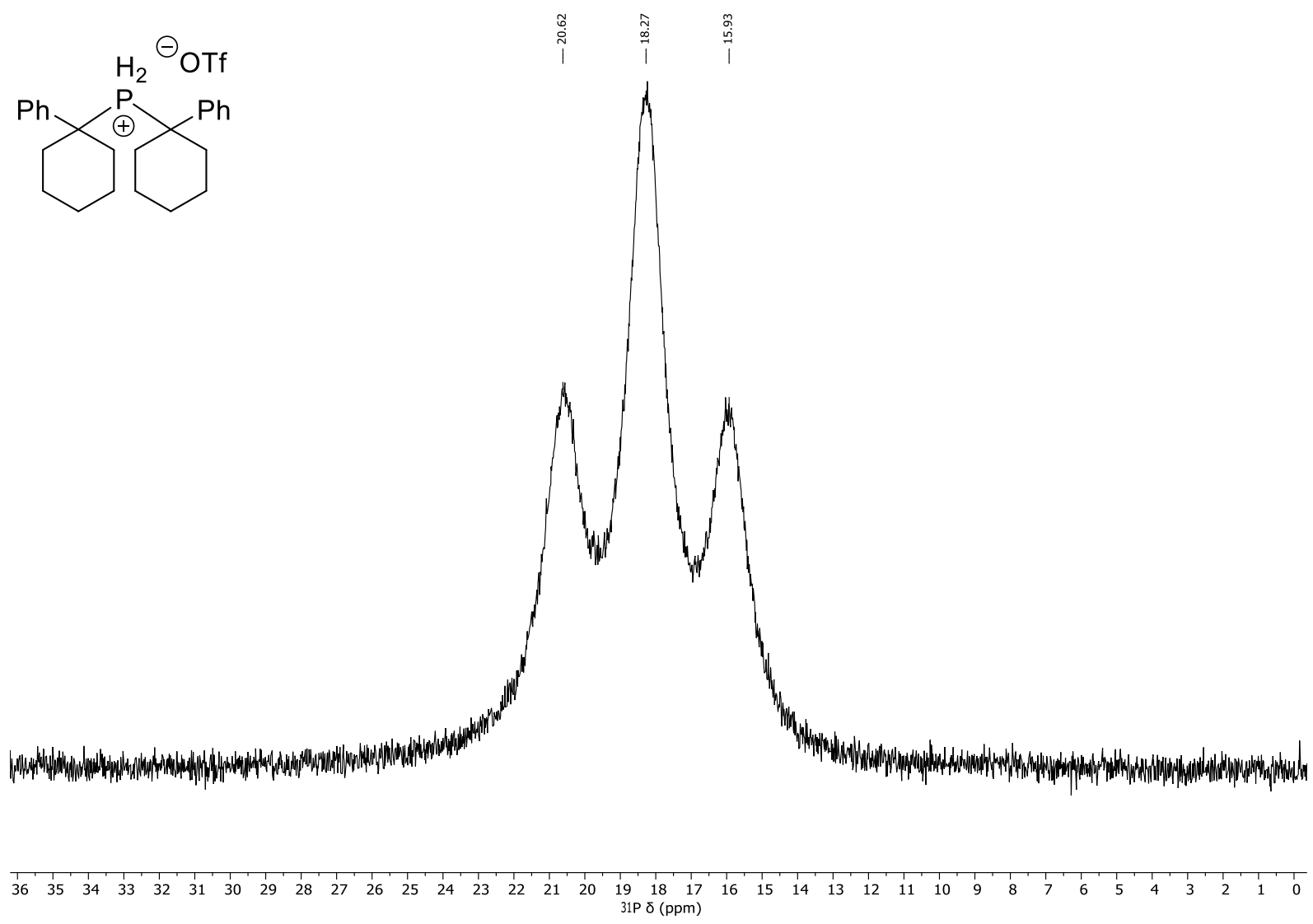

S144 
Di-(1-Adamantyl)phosphonium triflate (1k) - ${ }^{1} \mathrm{H}$ NMR $\left(500 \mathrm{MHz}, \mathrm{CDCl}_{3}\right)$
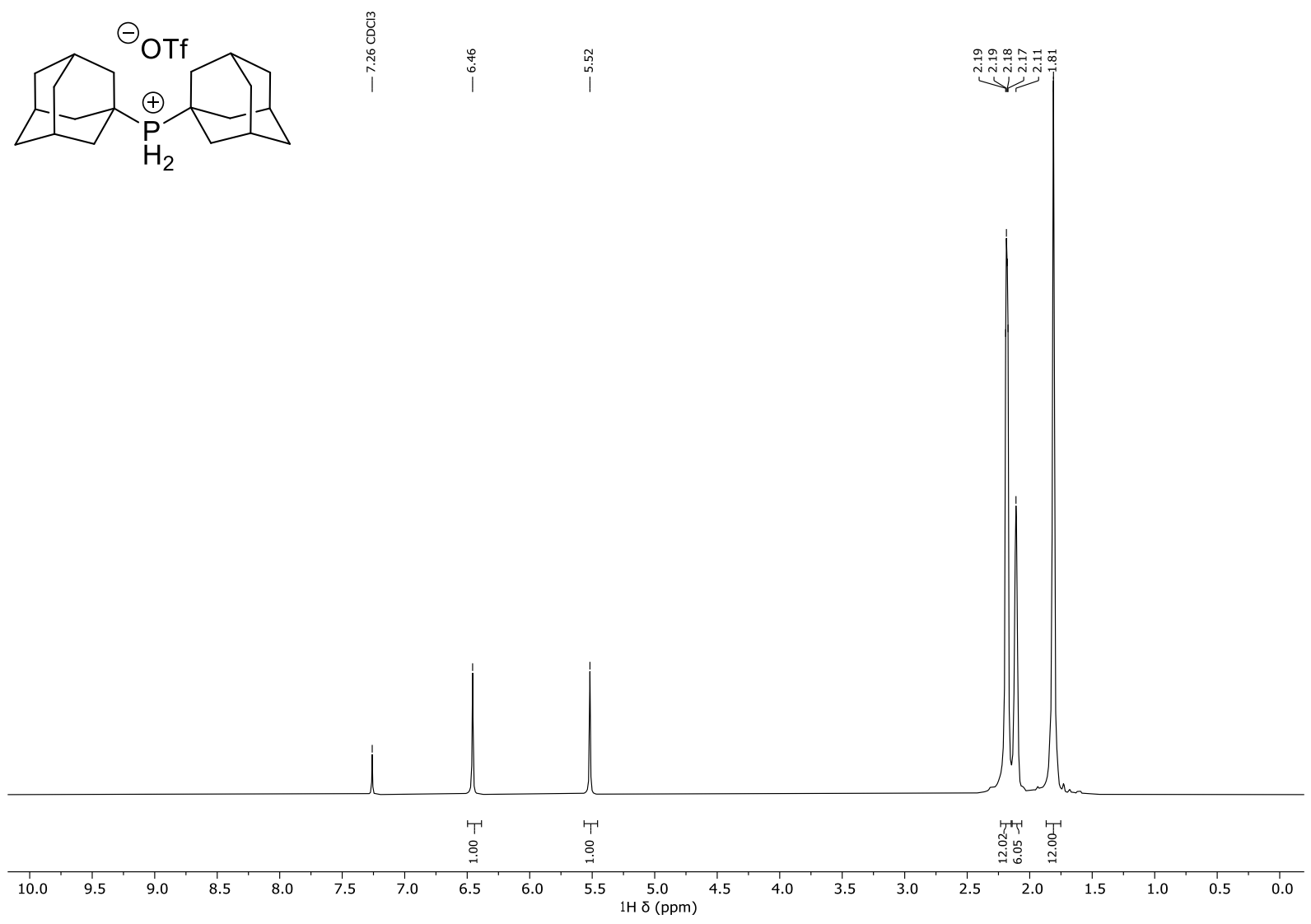

Di-(1-Adamantyl)phosphonium triflate (1k) - ${ }^{13} \mathrm{C}\left\{{ }^{1} \mathrm{H}\right\}$ NMR $\left(126 \mathrm{MHz}, \mathrm{CDCl}_{3}\right)$
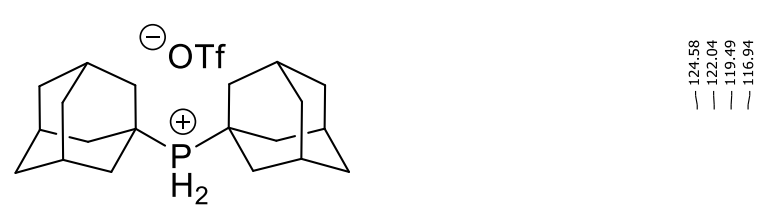
Di-(1-Adamantyl)phosphonium triflate (1k) $\left.{ }^{19} \mathrm{~F} \mathrm{NMR} \mathrm{(470} \mathrm{MHz}, \mathrm{CDCl}_{3}\right)$
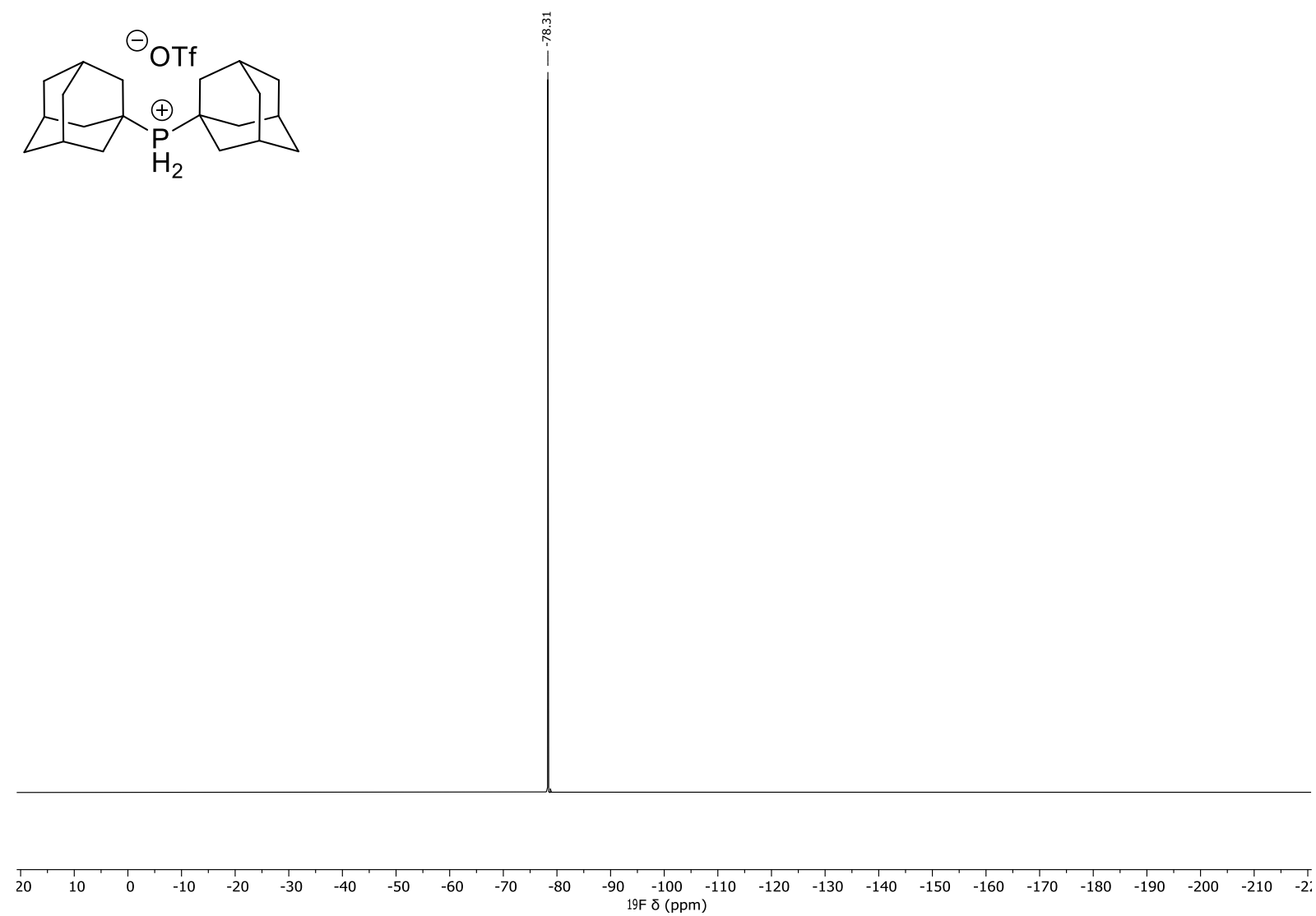

Di-(1-Adamantyl)phosphonium triflate $(1 \mathrm{k}){ }^{31} \mathrm{P}\left\{{ }^{1} \mathrm{H}\right\} \mathrm{NMR}\left(202 \mathrm{MHz}, \mathrm{CDCl}_{3}\right)$<smiles>[O-]C12CC3CC(CC(C3)C1)C2</smiles> 
Di-(1-Adamantyl)phosphonium triflate (1k) ${ }^{31}$ P NMR (202 MHz, $\mathrm{CDCl}_{3}$ )<smiles>[O]C12CC3CC(CC(C3)C1)C2</smiles>

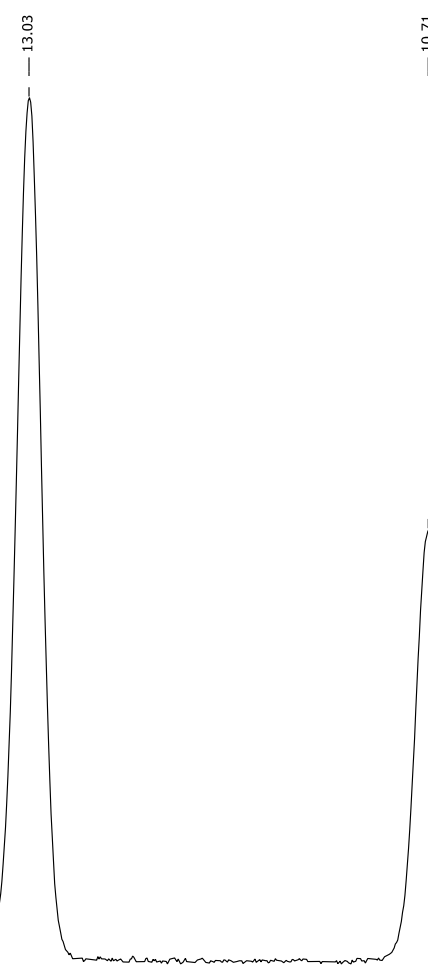

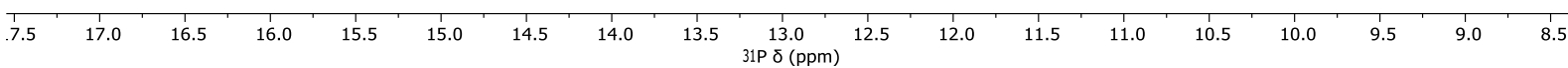


Di-(2-methyl-2-adamantyl)phosphonium triflate (1I) - ${ }^{1} \mathrm{H}$ NMR $\left(500 \mathrm{MHz}, \mathrm{CDCl}_{3}\right.$ )
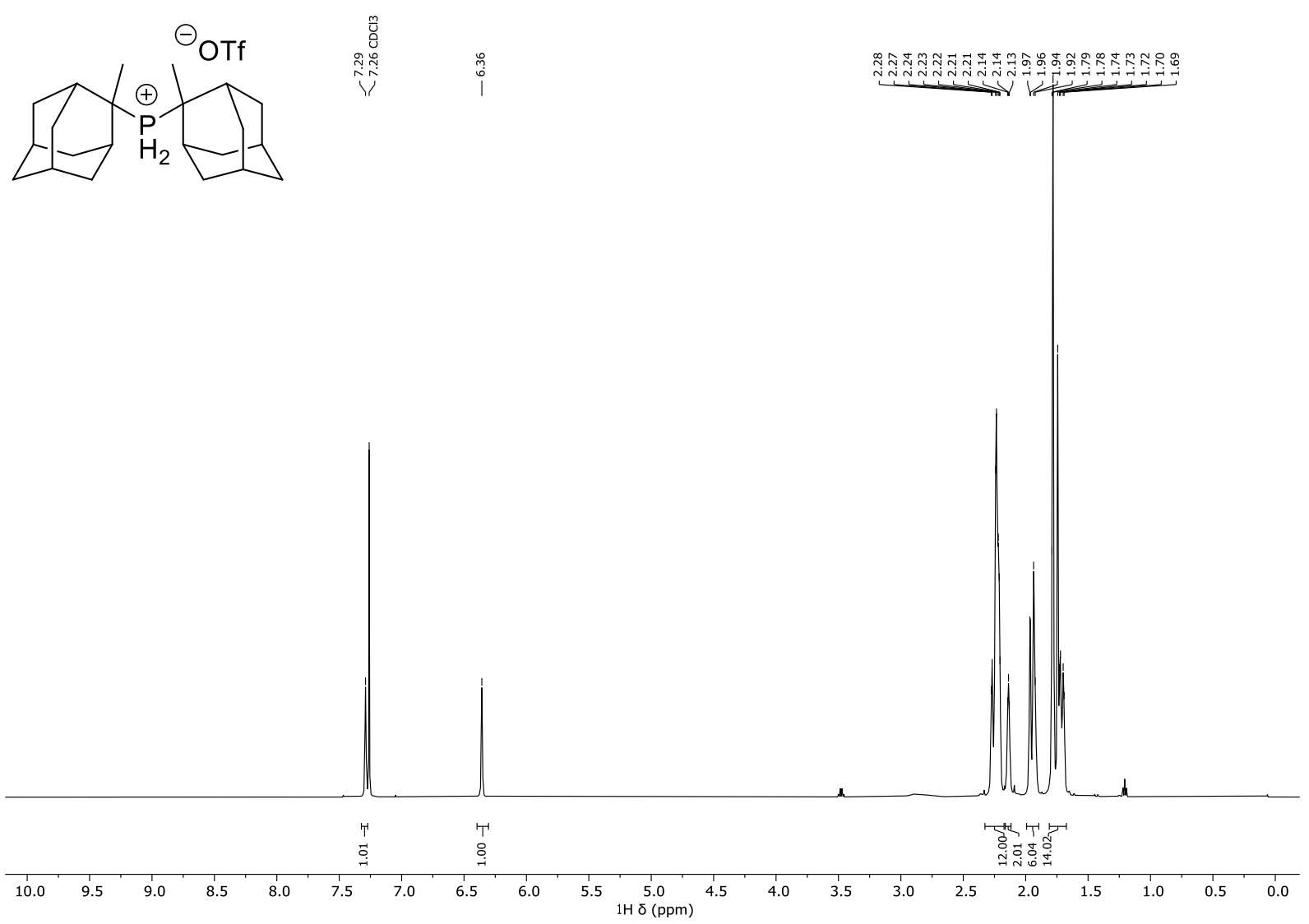

Di-(2-methyl-2-adamantyl)phosphonium triflate (1l) - ${ }^{13} \mathrm{C}\left\{{ }^{1} \mathrm{H}\right\}$ NMR (126 MHz, $\left.\mathrm{CDCl}_{3}\right)$
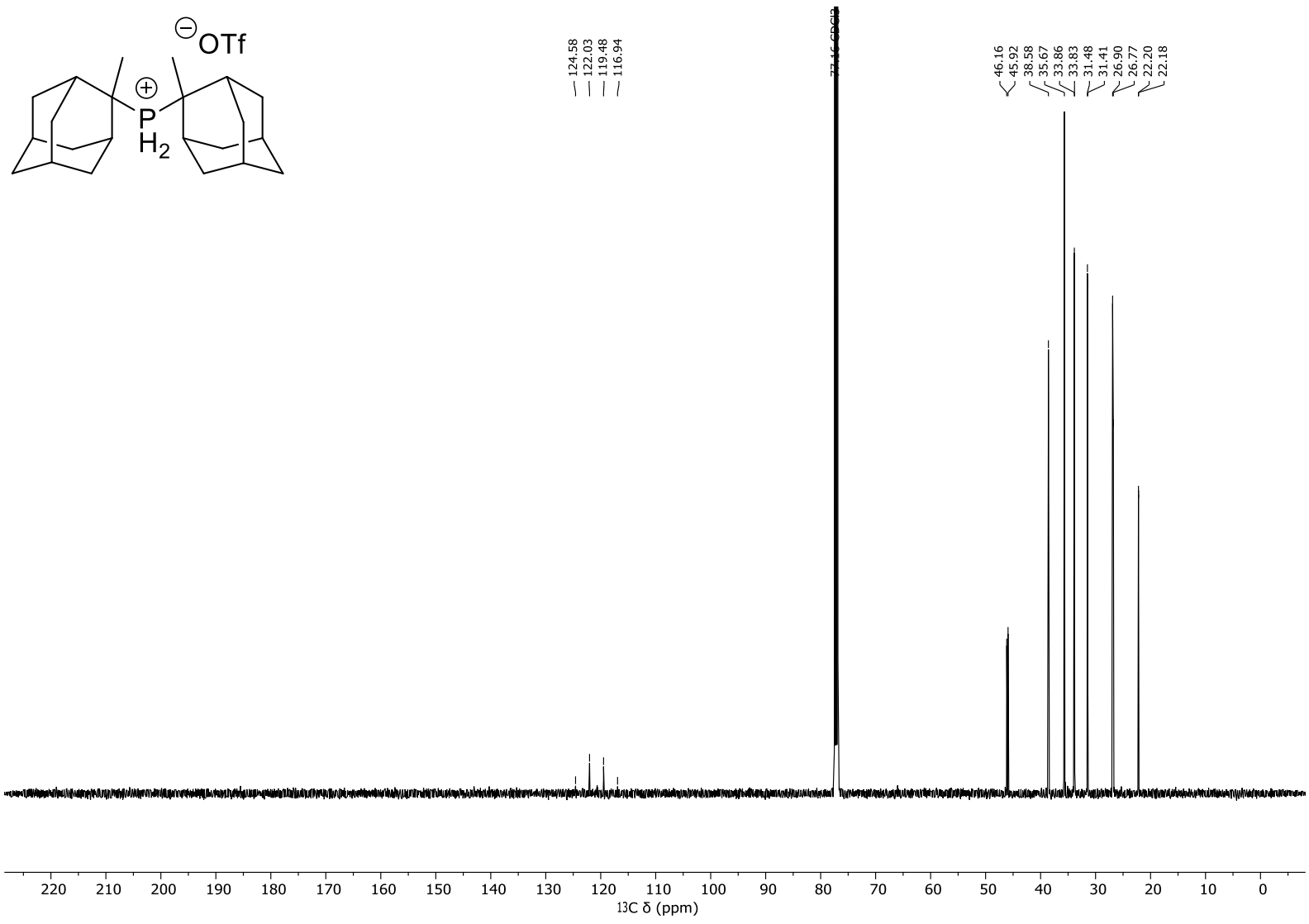
Di-(2-methyl-2-adamantyl)phosphonium triflate (1I) ${ }^{19} \mathrm{~F} \mathrm{NMR}\left(470 \mathrm{MHz}, \mathrm{CDCl}_{3}\right)$
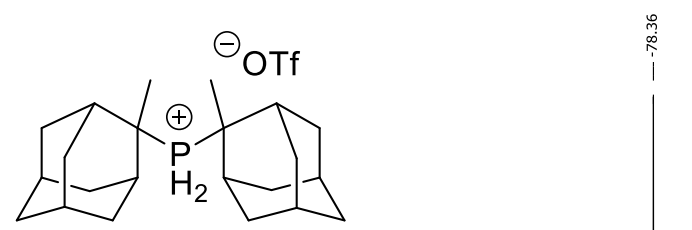

\begin{tabular}{llllllllllllllllllllllllllll}
\hline 20 & 10 & 0 & -10 & -20 & -30 & -40 & -50 & -60 & -70 & -80 & -90 & -100 & -110 & -120 & -130 & -140 & -150 & -160 & -170 & -180 & -190 & -200 & -210 & $-2:$
\end{tabular}

Di-(2-methyl-2-adamantyl)phosphonium triflate (1I) ${ }^{31} \mathrm{P}\left\{{ }^{1} \mathrm{H}\right\} \mathrm{NMR}\left(202 \mathrm{MHz}, \mathrm{CDCl}_{3}\right)$

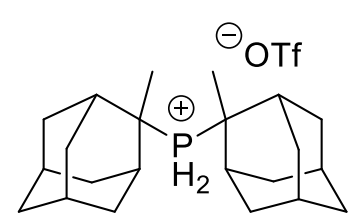


Di-(2-methyl-2-adamantyl)phosphonium triflate (1) ${ }^{31} \mathrm{P} \mathrm{NMR}\left(202 \mathrm{MHz}, \mathrm{CDCl}_{3}\right.$ )<smiles>CC1(PC2(O)C3CC4CC(C3)CC2C4)C2CC3CC(C2)CC1C3</smiles>

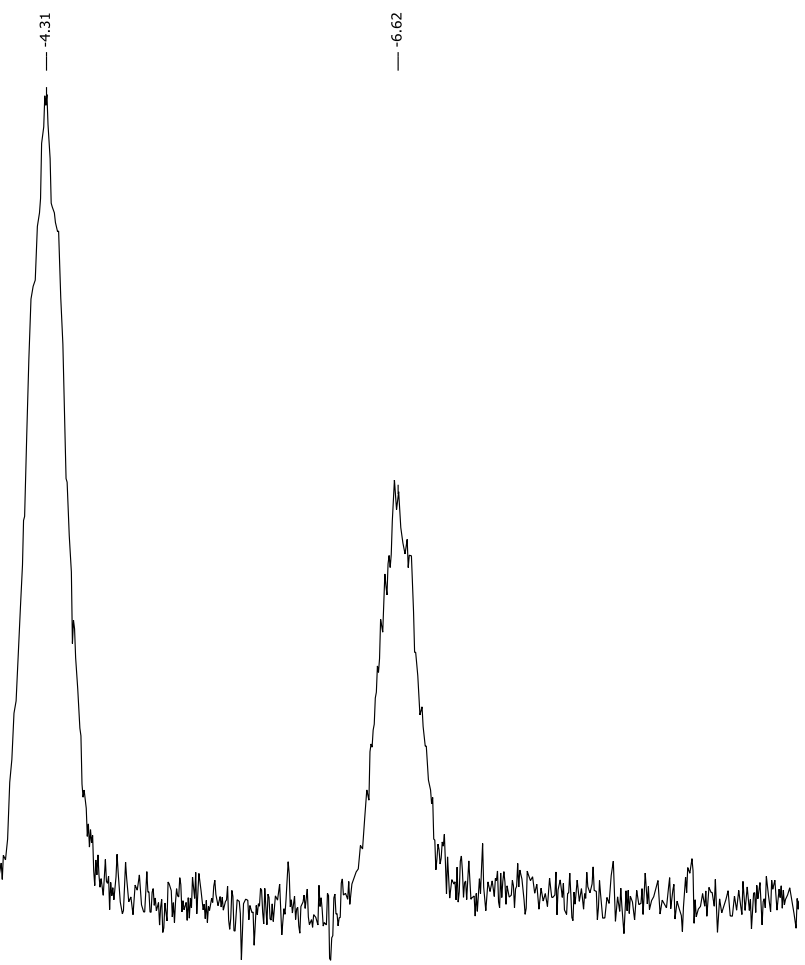

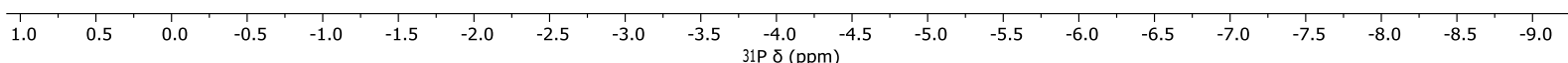


Dicedrylphosphonium triflate $(1 \mathrm{~m})-{ }^{1} \mathrm{H}$ NMR $\left(500 \mathrm{MHz}, \mathrm{CDCl}_{3}\right)$

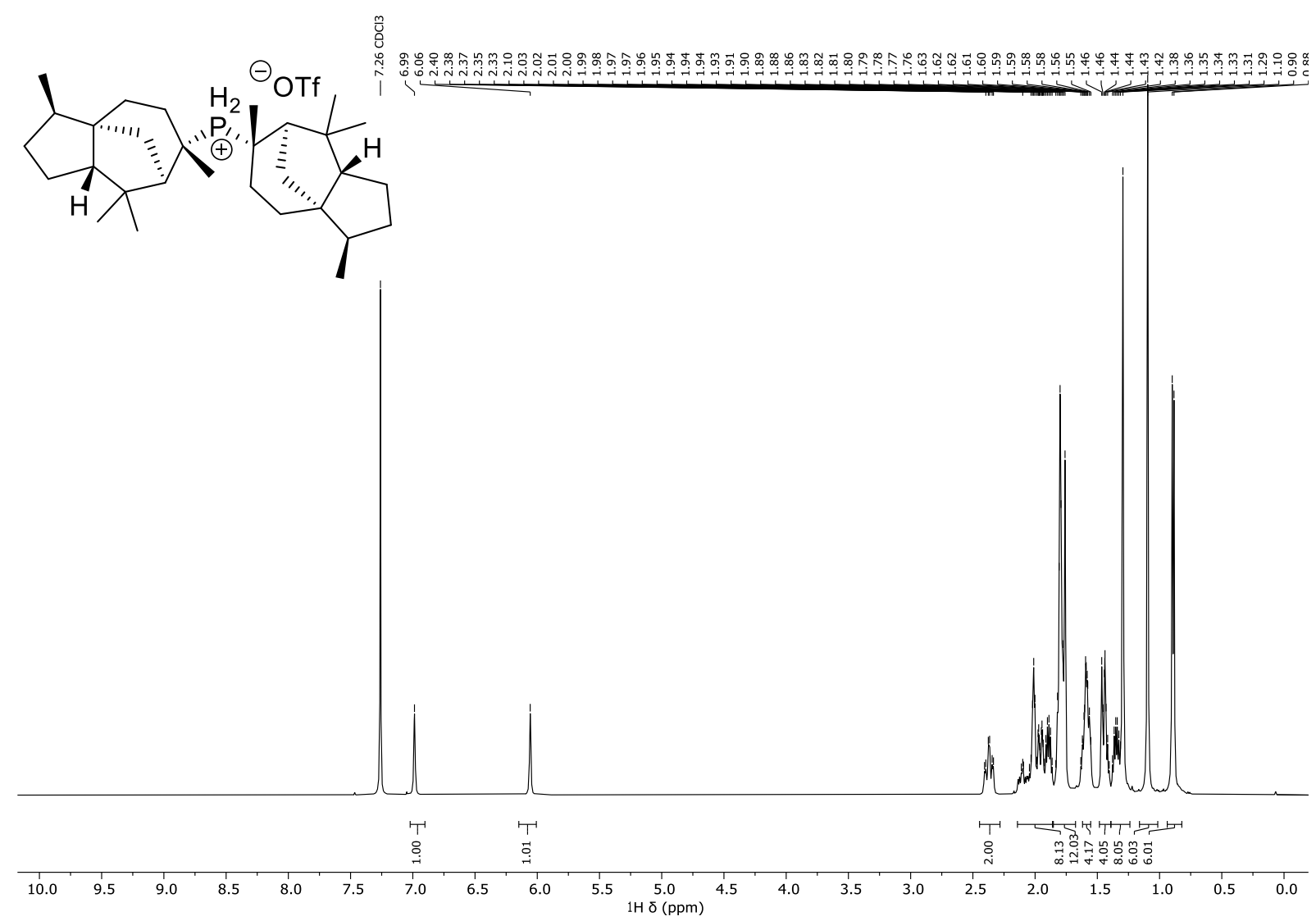

Dicedrylphosphonium triflate $(1 \mathrm{~m})-{ }^{13} \mathrm{C}\left\{{ }^{1} \mathrm{H}\right\} \mathrm{NMR}\left(126 \mathrm{MHz}, \mathrm{CDCl}_{3}\right)$

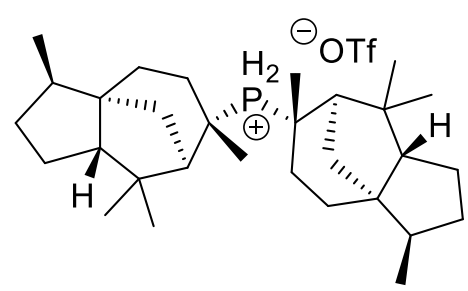

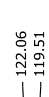

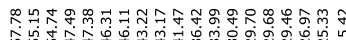

|

$\begin{array}{lllllllllllllllllllllllllll}220 & 210 & 200 & 190 & 180 & 170 & 160 & 150 & 140 & 130 & 120 & 110 & 100 & 90 & 80 & 70 & 60 & 50 & 40 & 30 & 20 & 10 & 0\end{array}$ 
Dicedrylphosphonium triflate $(1 \mathrm{~m}){ }^{19} \mathrm{~F} N \mathrm{NM}\left(470 \mathrm{MHz}, \mathrm{CDCl}_{3}\right)$
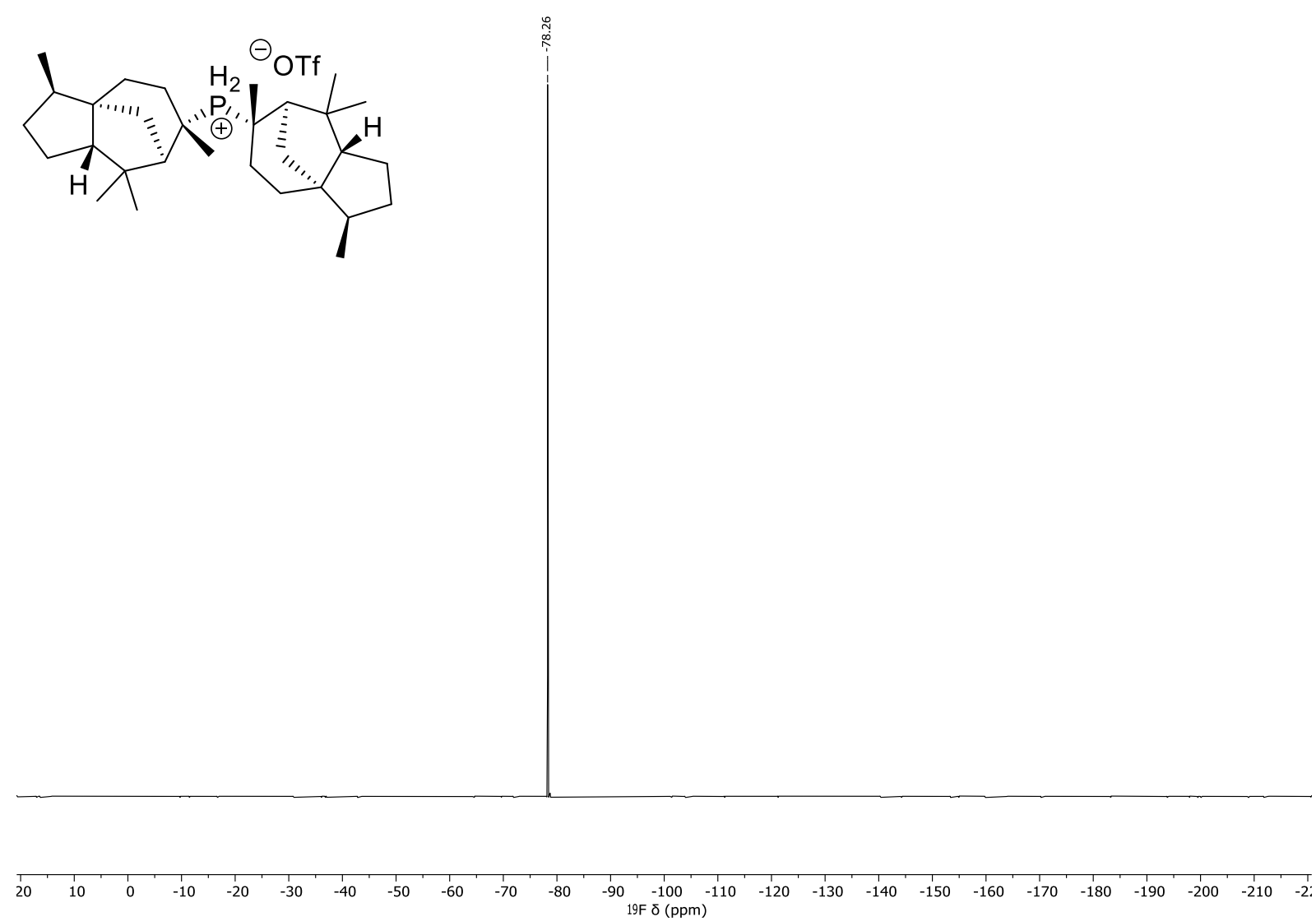

Dicedrylphosphonium triflate $(1 \mathrm{~m}){ }^{31} \mathrm{P}\left\{{ }^{1} \mathrm{H}\right\} \mathrm{NMR}\left(202 \mathrm{MHz}, \mathrm{CDCl}_{3}\right)$

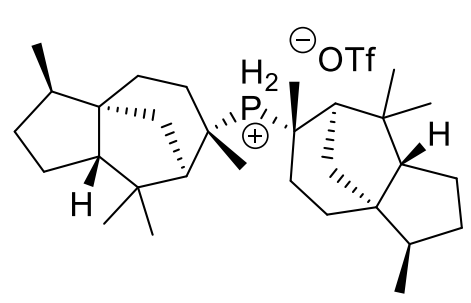


Dicedrylphosphonium triflate (1m) ${ }^{31} \mathrm{P}$ NMR $\left(202 \mathrm{MHz}, \mathrm{CDCl}_{3}\right)$
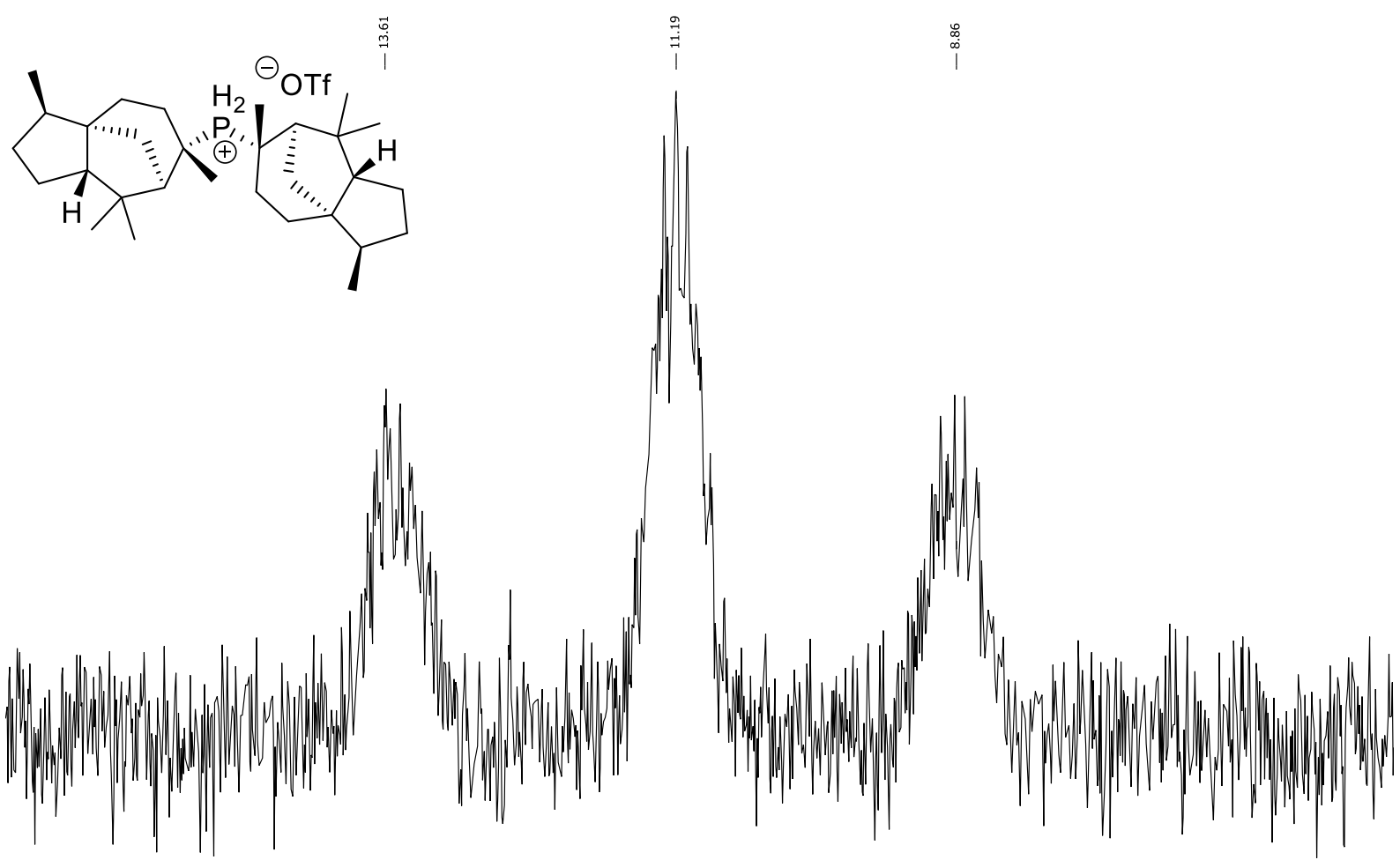

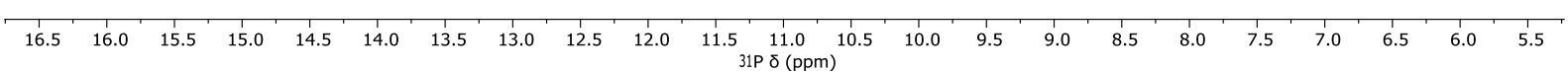


Tribenzhydrylphosphonium triflate (1n) - ${ }^{1} \mathrm{H}$ NMR (500 MHz, $\left.\mathrm{CDCl}_{3}\right)$

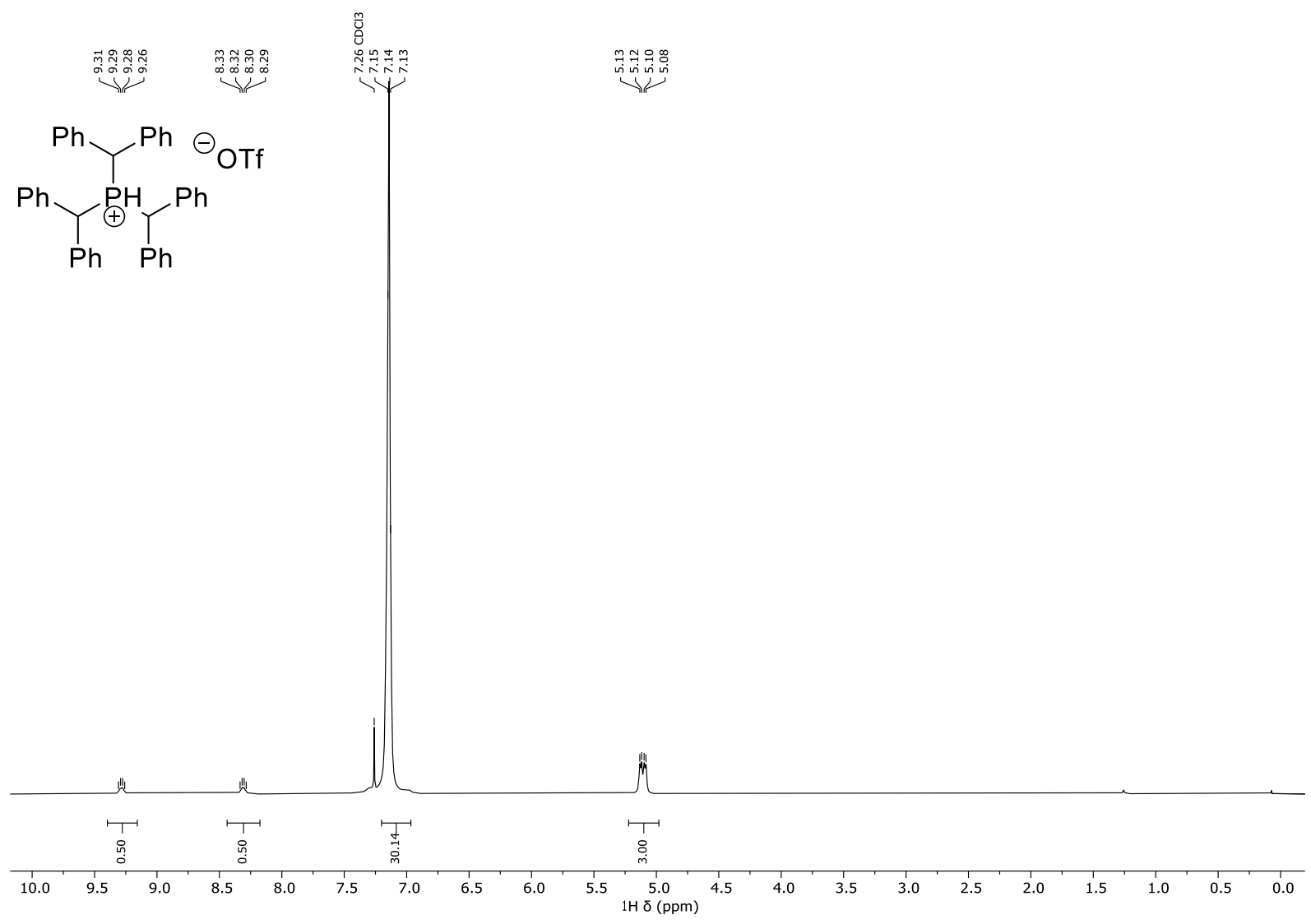

Tribenzhydrylphosphonium triflate $(1 \mathrm{n})-{ }^{13} \mathrm{C}\left\{{ }^{1} \mathrm{H}\right\} \mathrm{NMR}\left(126 \mathrm{MHz}, \mathrm{CDCl}_{3}\right)$
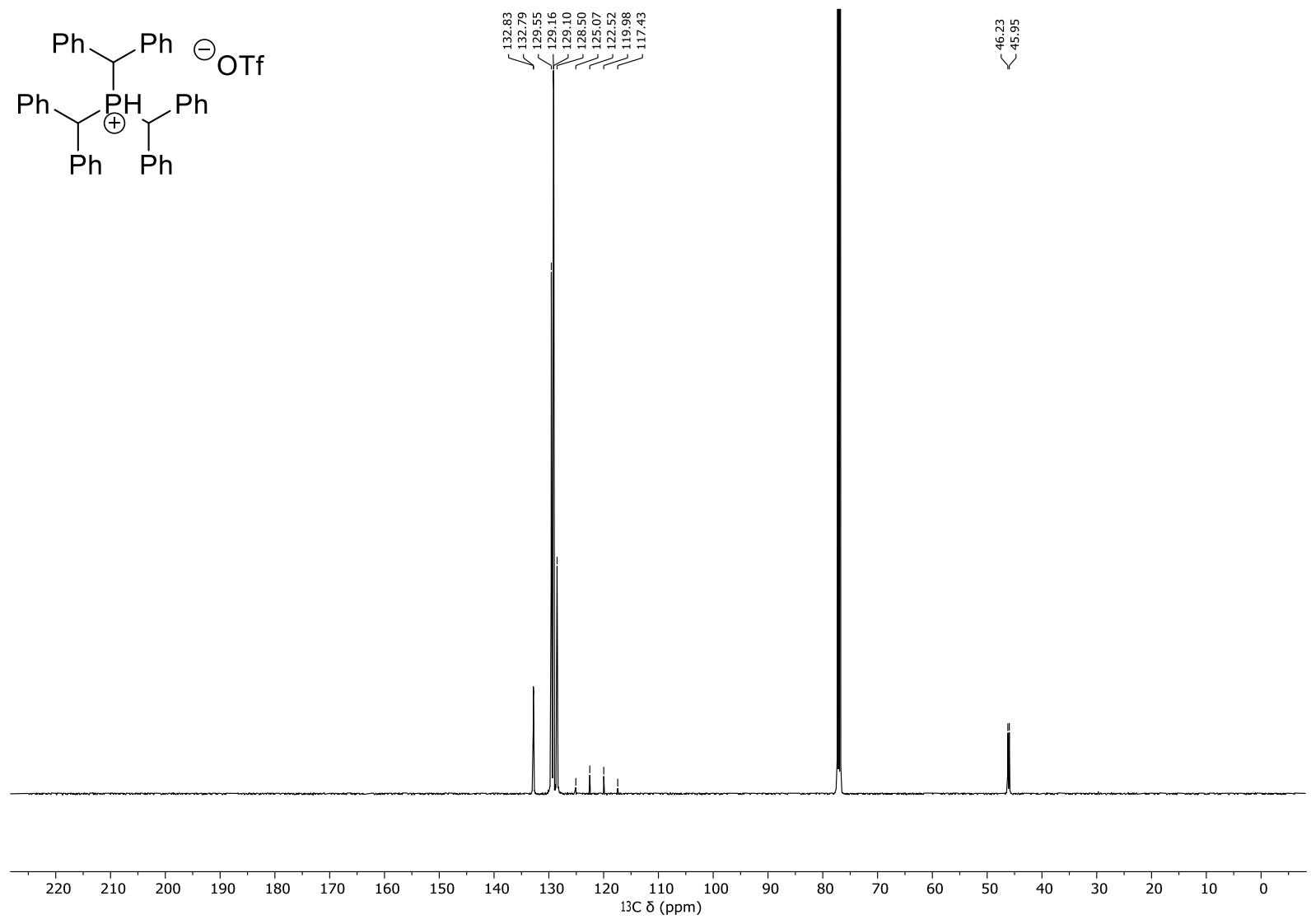

S154 
Tribenzhydrylphosphonium triflate (1n) ${ }^{19} \mathrm{~F} \mathrm{NMR} \mathrm{(376} \mathrm{MHz,} \mathrm{CDCl}_{3}$ )
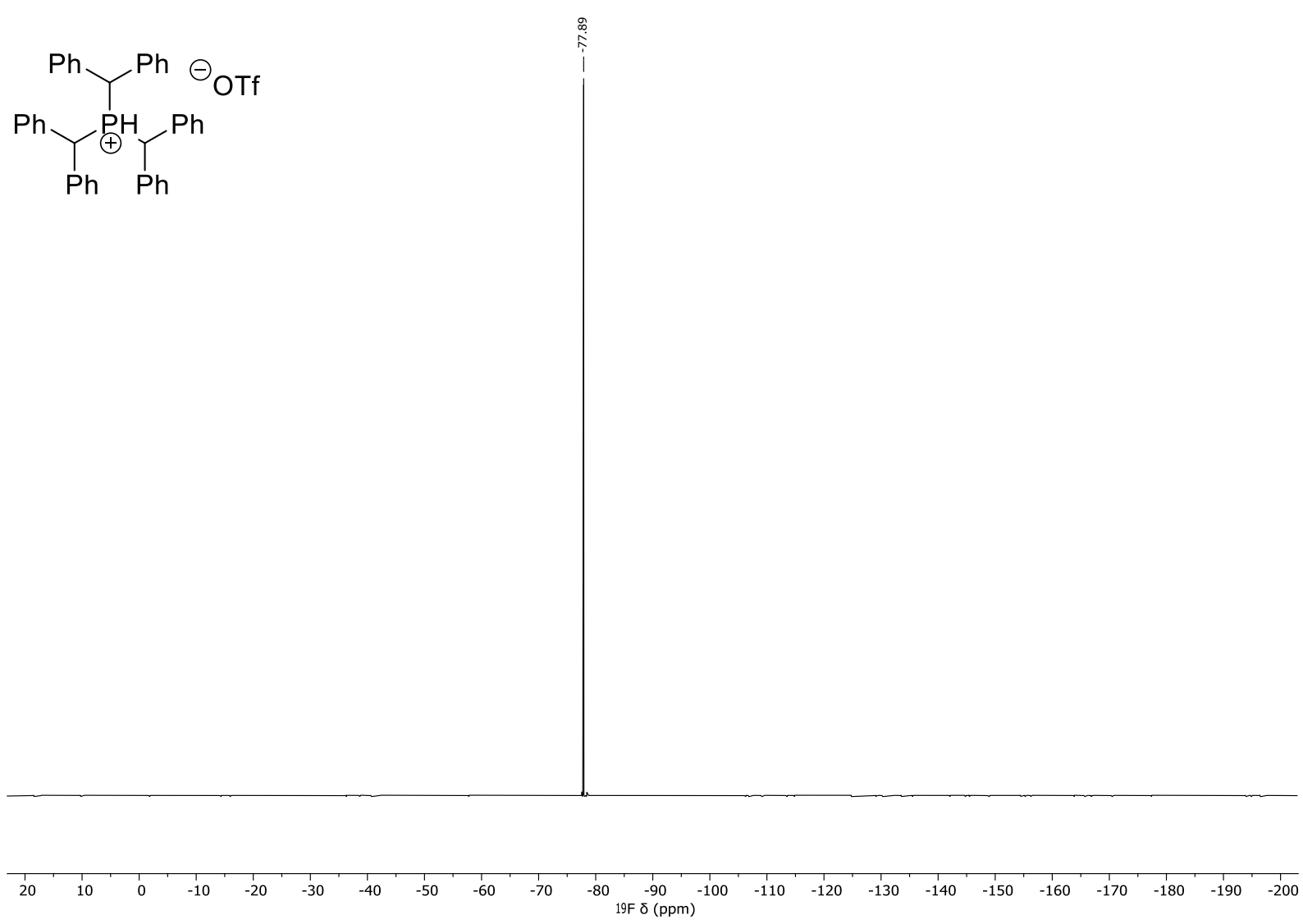

Tribenzhydrylphosphonium triflate $(1 \mathrm{n}){ }^{31} \mathrm{P}\left\{{ }^{1} \mathrm{H}\right\} \mathrm{NMR}\left(162 \mathrm{MHz}, \mathrm{CDCl}_{3}\right)$<smiles>c1ccc(-c2ccccc2[Pb](c2ccccc2)c2ccccc2)cc1</smiles>

\begin{tabular}{llllllll}
\hline & 1 \\
\hline & 150 & 100 & 0 & 1 & 1 & 1 & -1 \\
$31 \mathrm{P} \delta(\mathrm{ppm})$ & -50 & -100 & -150 & -1 \\
\hline
\end{tabular}


Tribenzhydrylphosphonium triflate (1n) $\left.{ }^{31} \mathrm{P} \mathrm{NMR} \mathrm{(162} \mathrm{MHz,} \mathrm{CDCl}_{3}\right)$

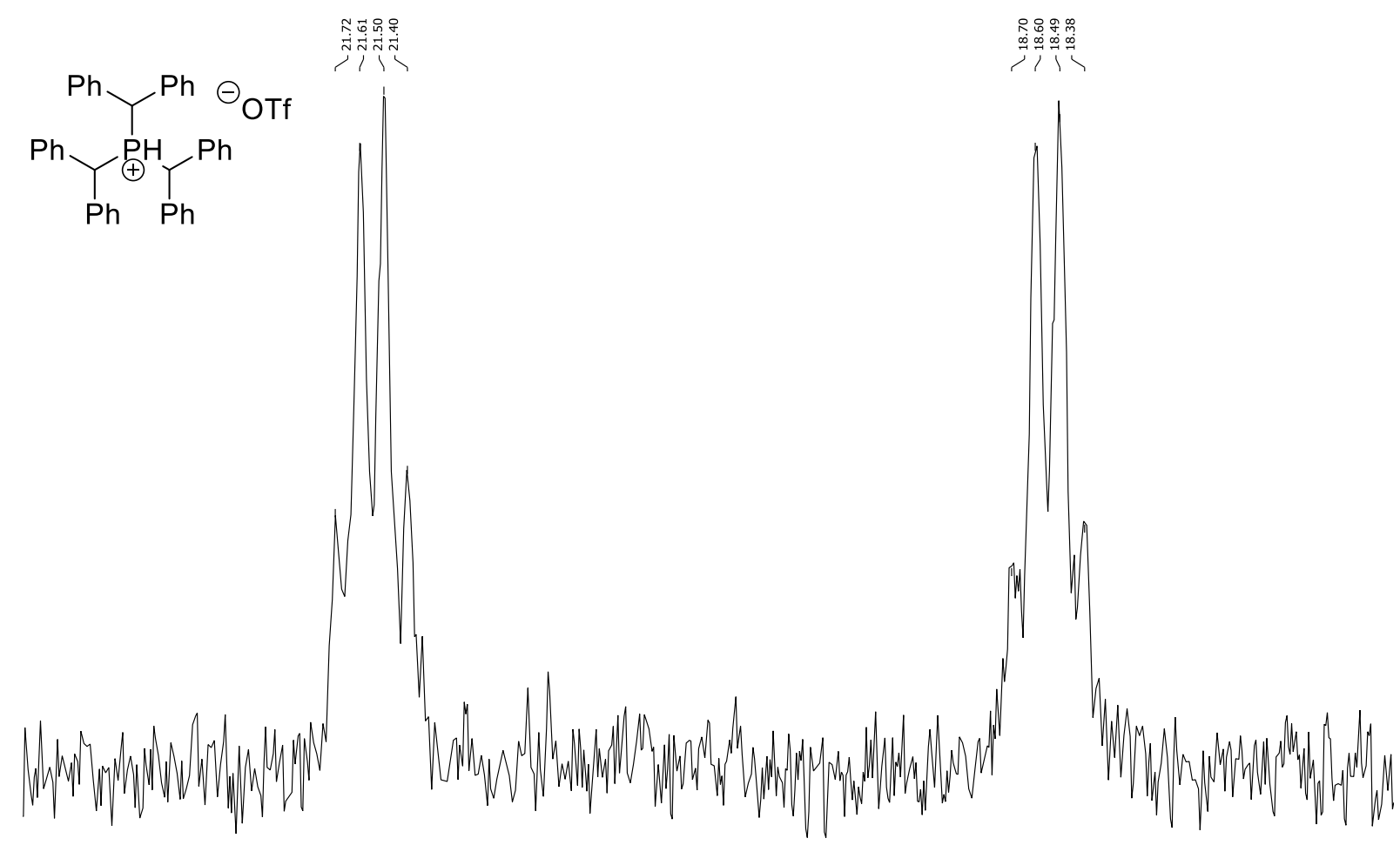

$\begin{array}{llllllllllllllllllllllllllllllllllllllllllll}23.0 & 22.8 & 22.6 & 22.4 & 22.2 & 22.0 & 21.8 & 21.6 & 21.4 & 21.2 & 21.0 & 20.8 & 20.6 & 20.4 & 20.2 & 20.0 & 19.8 & 19.6 & 19.4 & 19.2 & 19.0 & 18.8 & 18.6 & 18.4 & 18.2 & 18.0 & 17.8 & 17.6 & 17.4 & 17.2 & 1\end{array}$ $31 \mathrm{P} \delta(\mathrm{ppm})$ 
Bis-(2,4,4-trimethyl-2-pentyl)phosphonium triflate (1q) - ${ }^{1} \mathrm{H}$ NMR $\left(500 \mathrm{MHz}, \mathrm{CDCl}_{3}\right)$
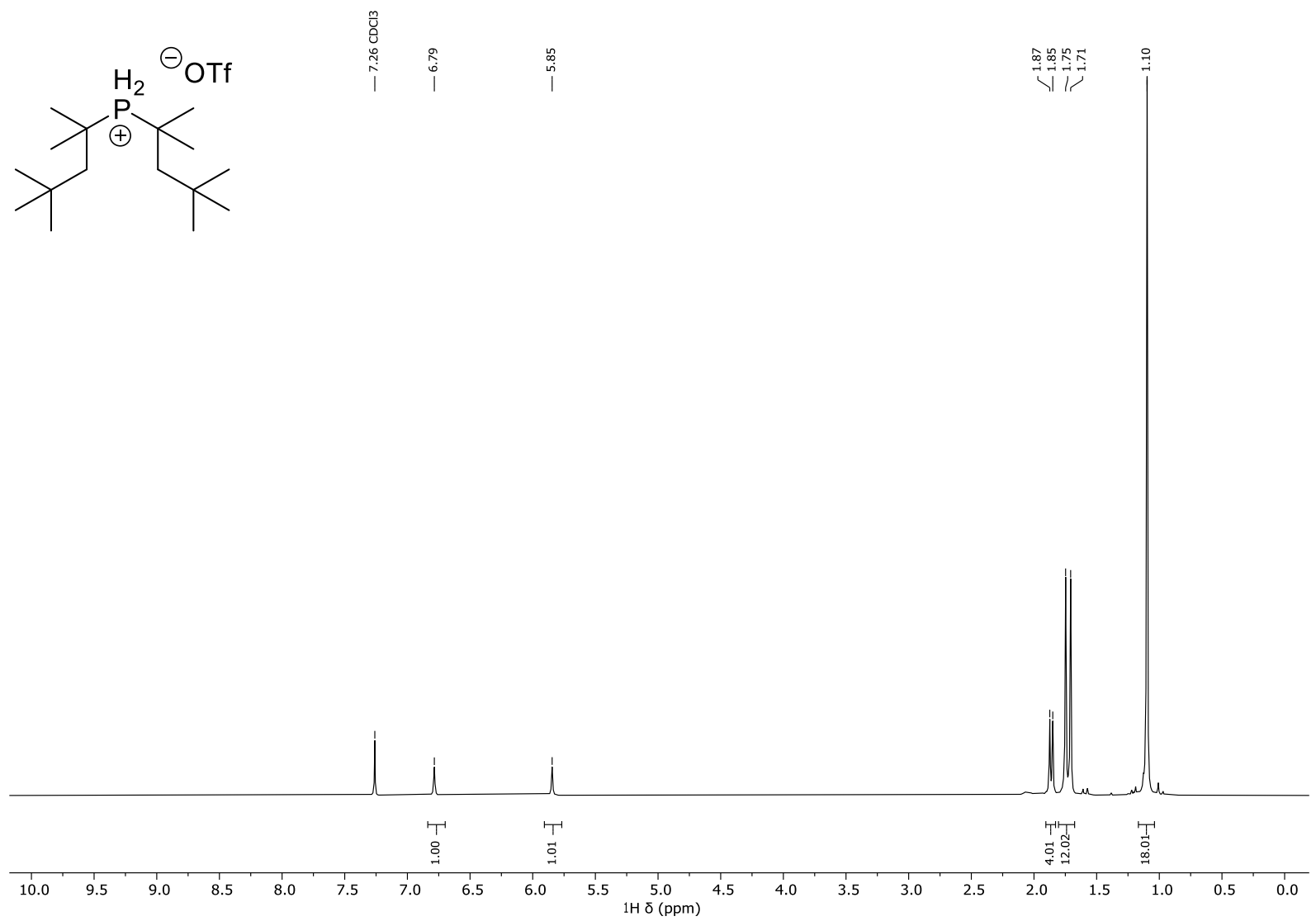

Bis-(2,4,4-trimethyl-2-pentyl)phosphonium triflate (1q) - ${ }^{13} \mathrm{C}\left\{{ }^{1} \mathrm{H}\right\} \mathrm{NMR}\left(126 \mathrm{MHz}, \mathrm{CDCl}_{3}\right)$
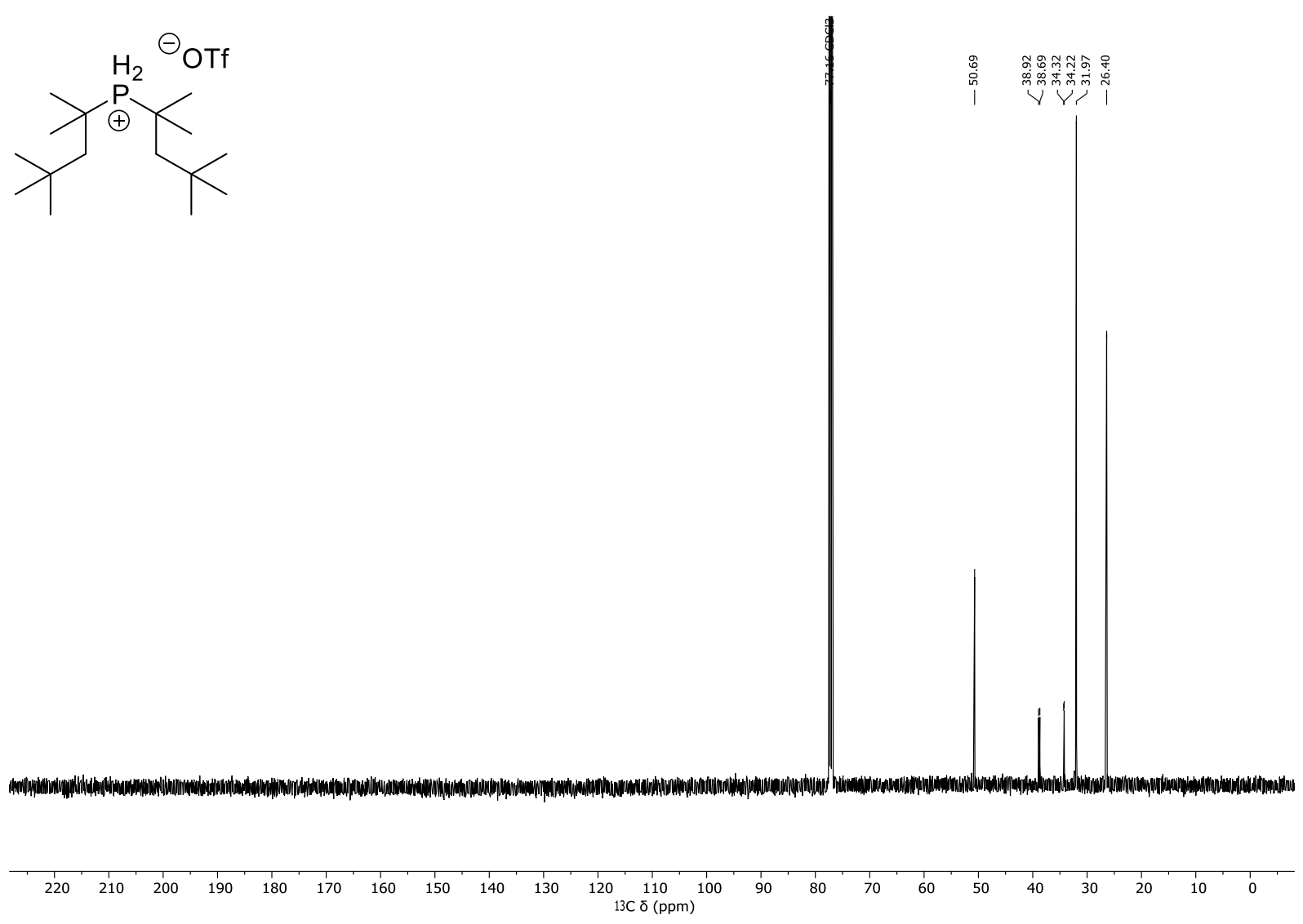

S157 


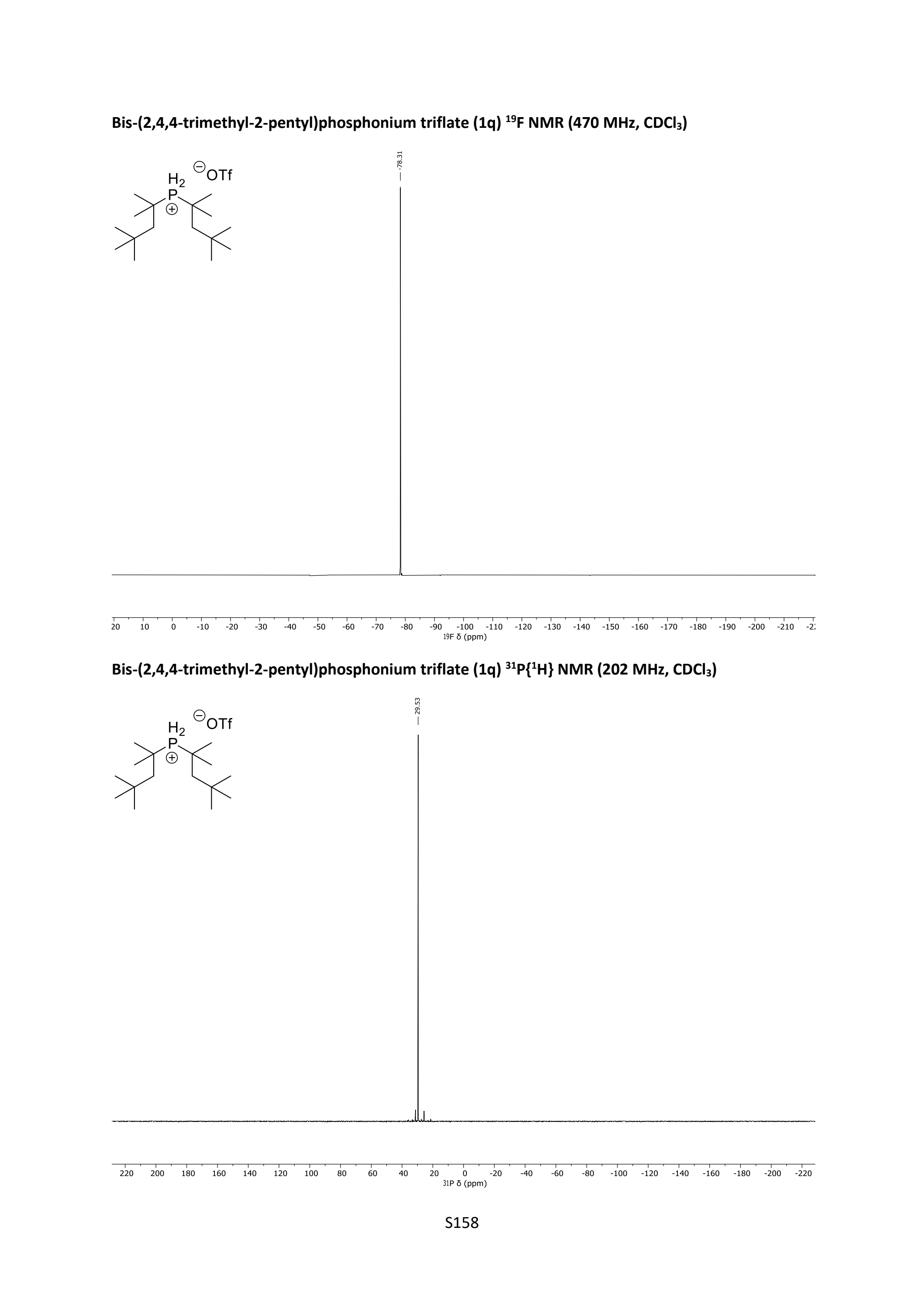


Bis-(2,4,4-trimethyl-2-pentyl)phosphonium triflate (1q) ${ }^{31}$ P NMR (202 MHz, $\left.\mathrm{CDCl}_{3}\right)$
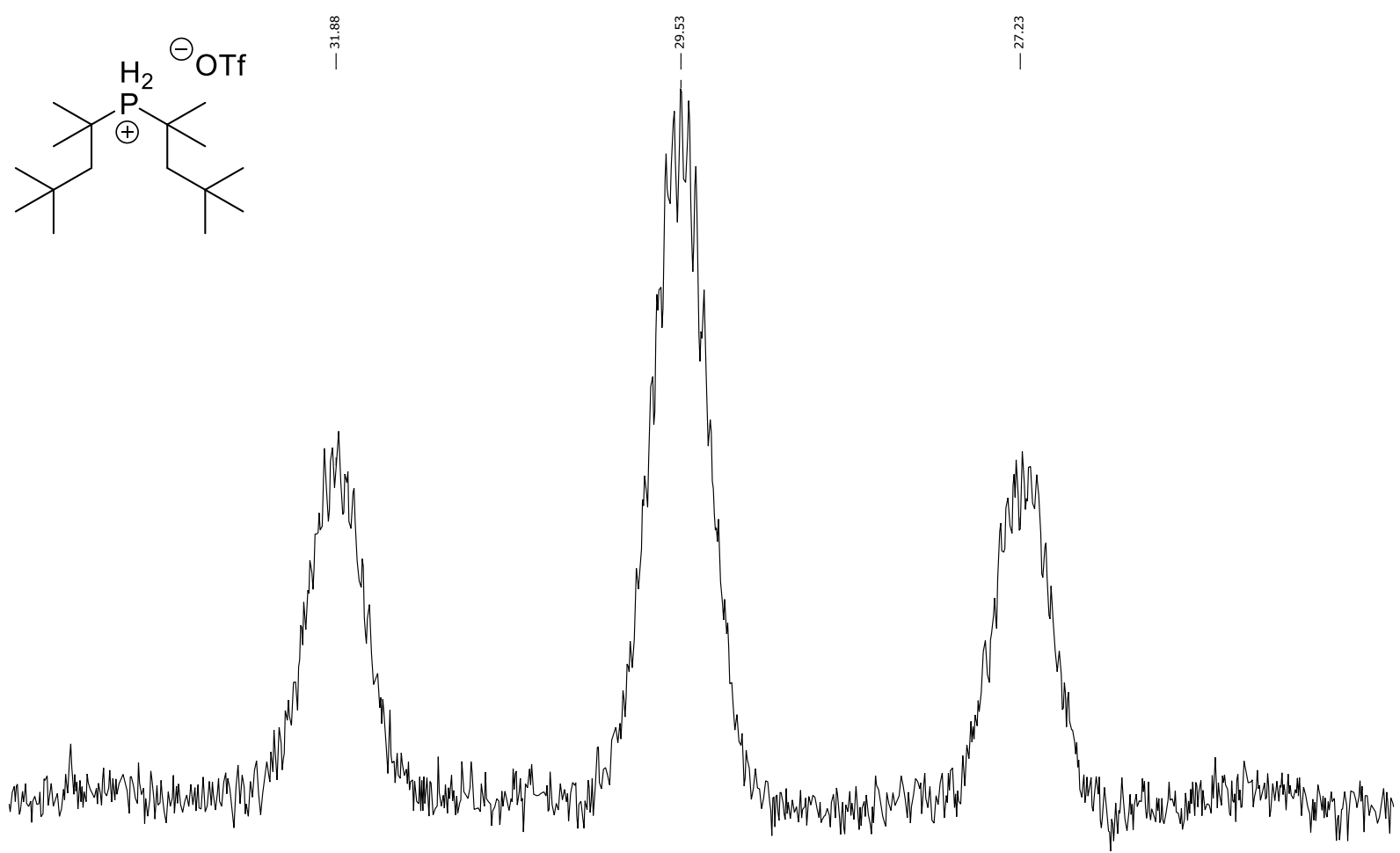

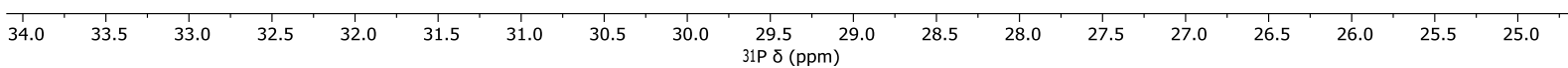


Di-(3-ethyl-3-pentyl)phosphine borane complex - ${ }^{1} \mathrm{H}$ NMR $\left(500 \mathrm{MHz}, \mathrm{CDCl}_{3}\right)$<smiles>[B]P(CC)C(CC)(CC)CC</smiles>

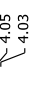

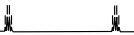
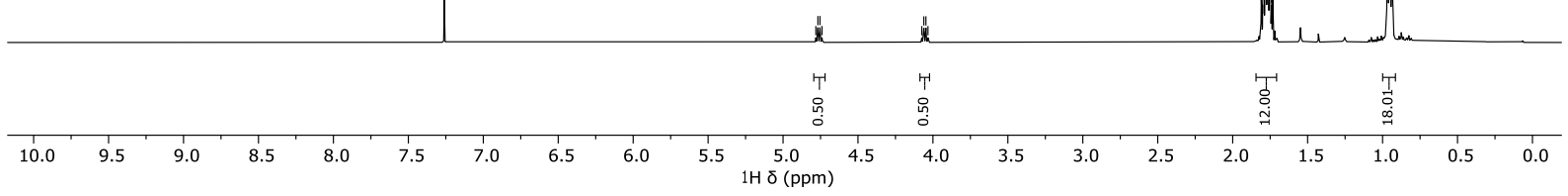

Di-(3-ethyl-3-pentyl)phosphine borane complex ${ }^{11} \mathrm{~B}\left\{{ }^{1} \mathrm{H}\right\}$ NMR $\left(160 \mathrm{MHz}, \mathrm{CDCl}_{3}\right)$<smiles>[B]P(CC)C(CC)(CC)CC</smiles> 
Di-(3-ethyl-3-pentyl)phosphine borane complex $-{ }^{13} \mathrm{C}\left\{{ }^{1} \mathrm{H}\right\}$ NMR $\left(126 \mathrm{MHz}, \mathrm{CDCl}_{3}\right)$
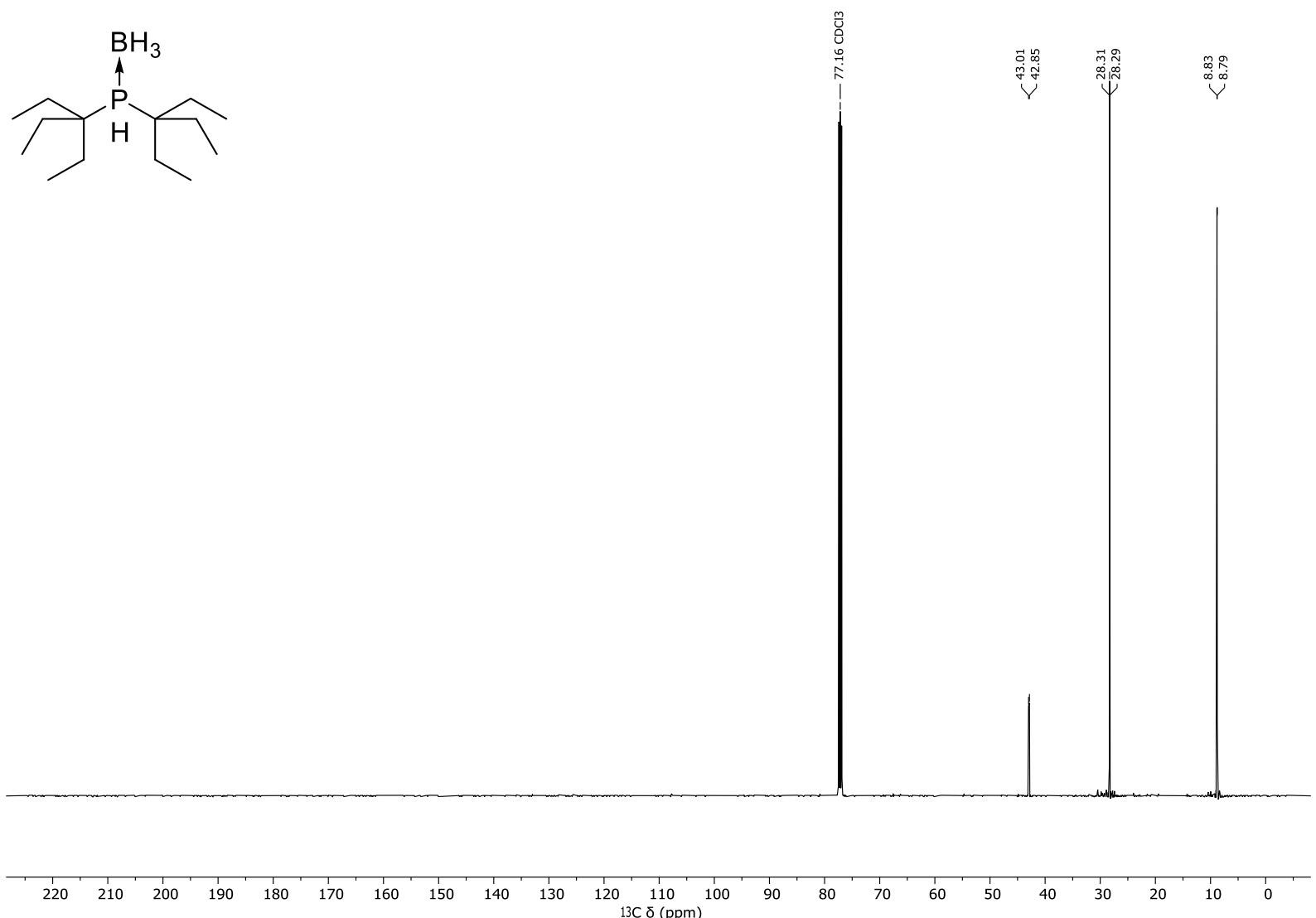

Di-(3-ethyl-3-pentyl)phosphine borane complex ${ }^{31} \mathrm{P}\left\{{ }^{1} \mathrm{H}\right\} \mathrm{NMR}\left(202 \mathrm{MHz}, \mathrm{CDCl}_{3}\right)$<smiles>[B]P(CC)C(CC)(CC)CC</smiles> 
Di-(3-ethyl-3-pentyl)phosphine borane complex ${ }^{31} \mathrm{P}$ NMR (202 MHz, $\mathrm{CDCl}_{3}$ )

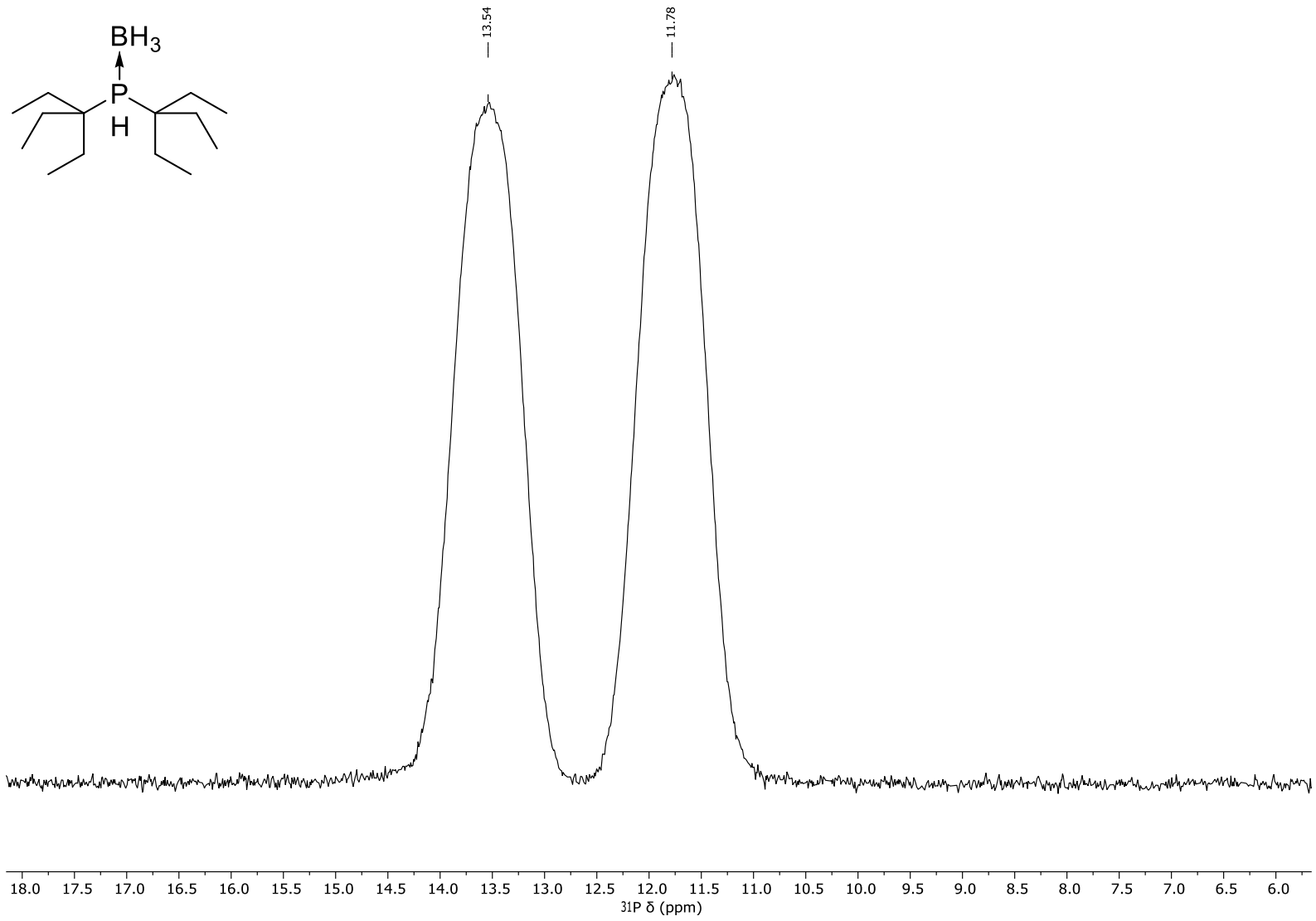


Di-(3-ethyl-3-pentyl)chlorophosphine - ${ }^{1} \mathrm{H}$ NMR $\left(500 \mathrm{MHz}, \mathrm{CDCl}_{3}\right)$<smiles>CCC(CC)(CC)P(Cl)C(CC)(CC)CC</smiles>

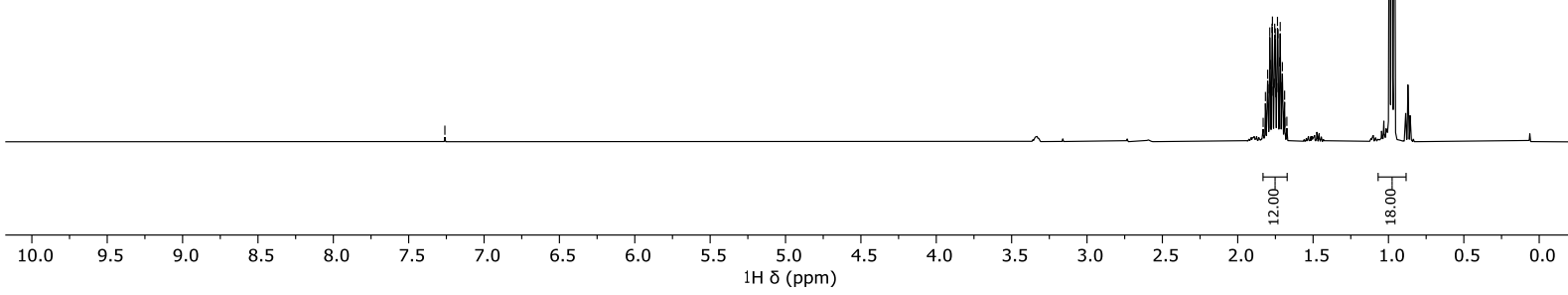

Di-(3-ethyl-3-pentyl)chlorophosphine $-{ }^{13} \mathrm{C}\left\{{ }^{1} \mathrm{H}\right\}$ NMR (126 MHz, $\left.\mathrm{CDCl}_{3}\right)$<smiles>CCC(CC)(CC)P(Cl)C(CC)(CC)CC</smiles>

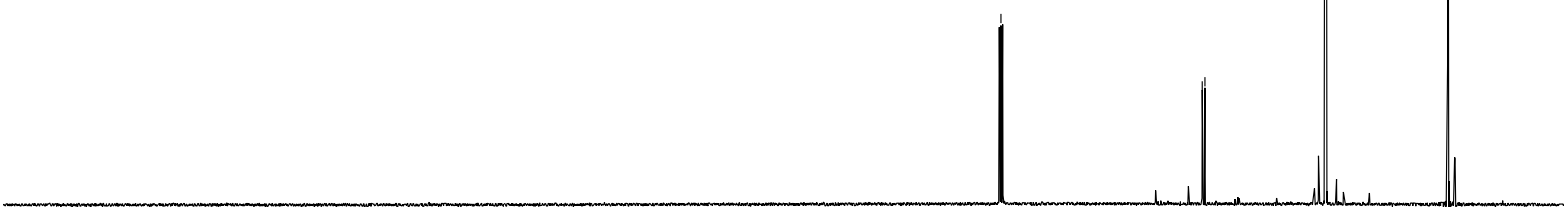

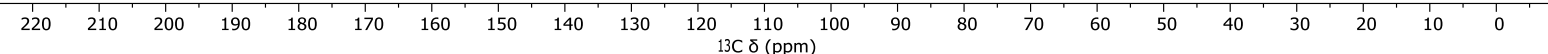


Di-(3-ethyl-3-pentyl)chlorophosphine ${ }^{31} \mathrm{P}\left\{{ }^{1} \mathrm{H}\right\}$ NMR $\left(202 \mathrm{MHz}, \mathrm{CDCl}_{3}\right)$<smiles>CCC(CC)(CC)P(Cl)C(CC)(CC)CC</smiles>

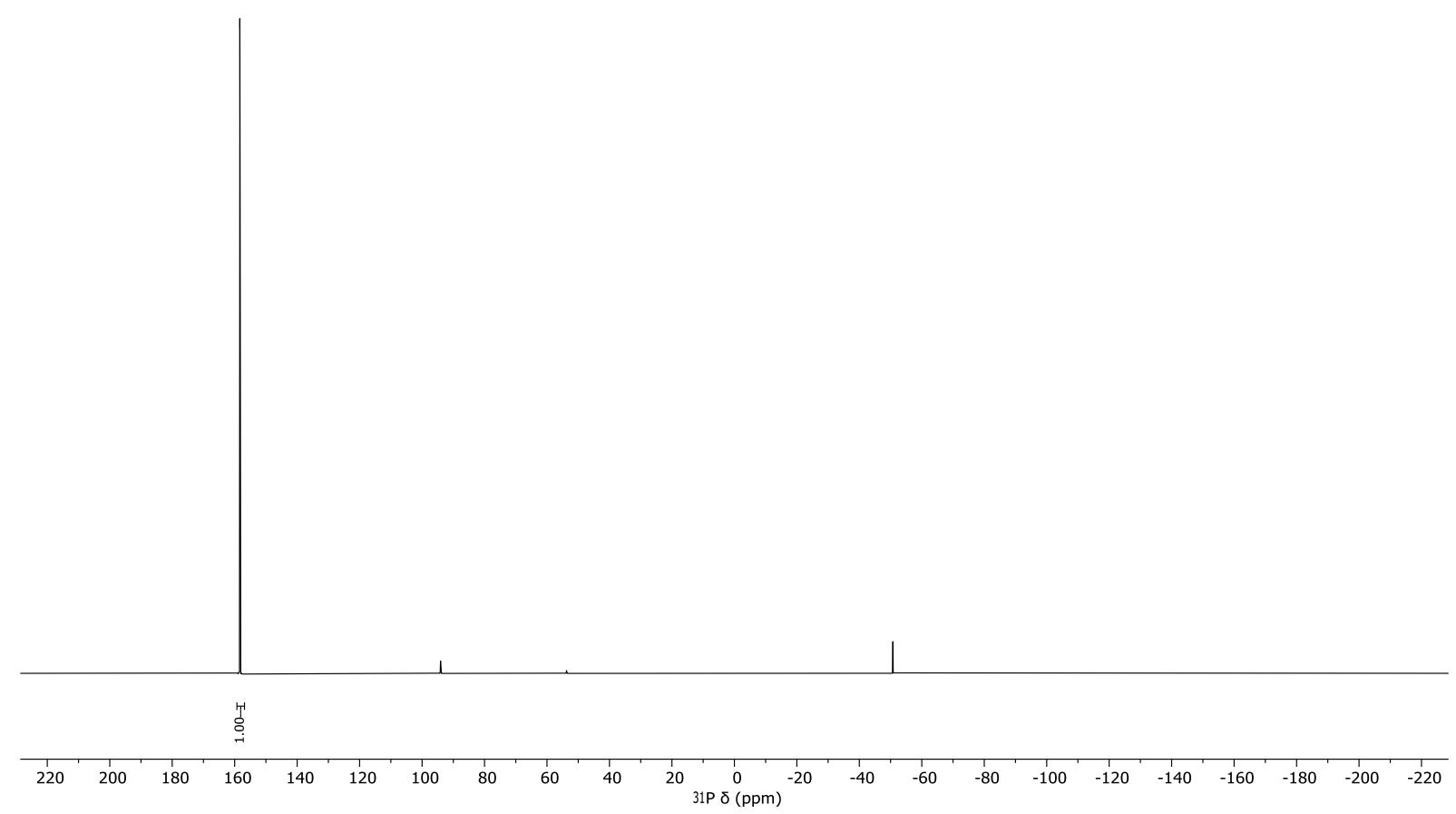

Di-(3-ethyl-3-pentyl)chlorophosphine ${ }^{31} \mathrm{P}$ NMR (202 MHz, $\left.\mathrm{CDCl}_{3}\right)$<smiles>CCC(CC)(CC)P(Cl)C(CC)(CC)CC</smiles>

$\begin{array}{rrrrrrrr}79.00 & 158.90 & 158.80 & 158.70 & 158.60 & 158.50 & 158.40 & 158.30 \\ 31 \mathrm{P} \delta(\mathrm{ppm})\end{array}$ 
Di-(3-ethyl-3-pentyl)phosphine oxide - ${ }^{1} \mathrm{H}$ NMR $\left(500 \mathrm{MHz}, \mathrm{CDCl}_{3}\right)$<smiles>CCC(CC)(CC)[PH](=O)C(CC)(CC)CC</smiles>

范

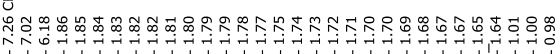

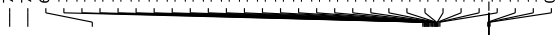

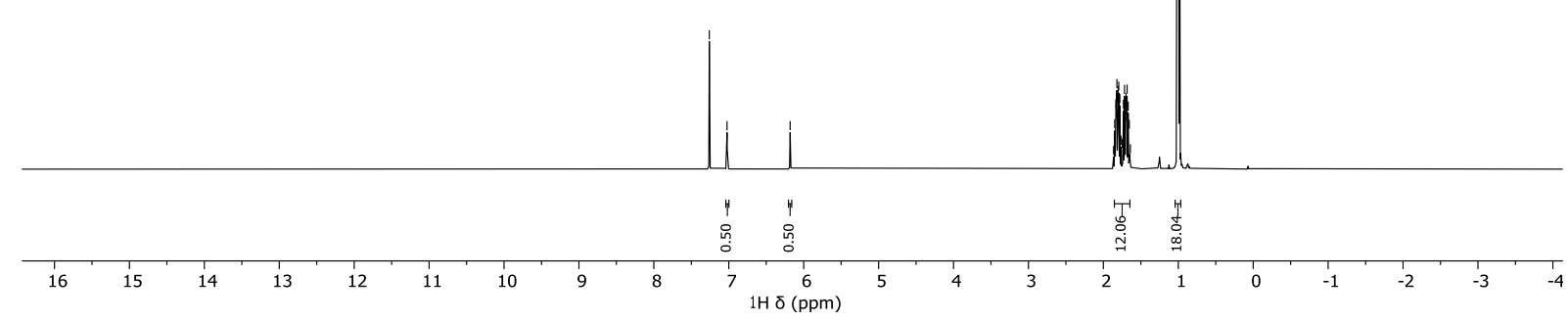

Di-(3-ethyl-3-pentyl)phosphine oxide $-{ }^{13} \mathrm{C}\left\{{ }^{1} \mathrm{H}\right\}$ NMR (126 MHz, $\left.\mathrm{CDCl}_{3}\right)$<smiles>CCC(CC)(CC)C(=O)C(CC)(CC)CC</smiles> 
Di-(3-ethyl-3-pentyl)phosphine oxide ${ }^{31} \mathrm{P}\left\{{ }^{1} \mathrm{H}\right\}$ NMR $\left(202 \mathrm{MHz}, \mathrm{CDCl}_{3}\right)$<smiles>CCC(CC)(CC)C(=O)C(CC)(CC)CC</smiles>

Di-(3-ethyl-3-pentyl)phosphine oxide ${ }^{31}$ P NMR (202 MHz, $\left.\mathrm{CDCl}_{3}\right)$<smiles>CCC(CC)(CC)C(=O)C(CC)(CC)CC</smiles>
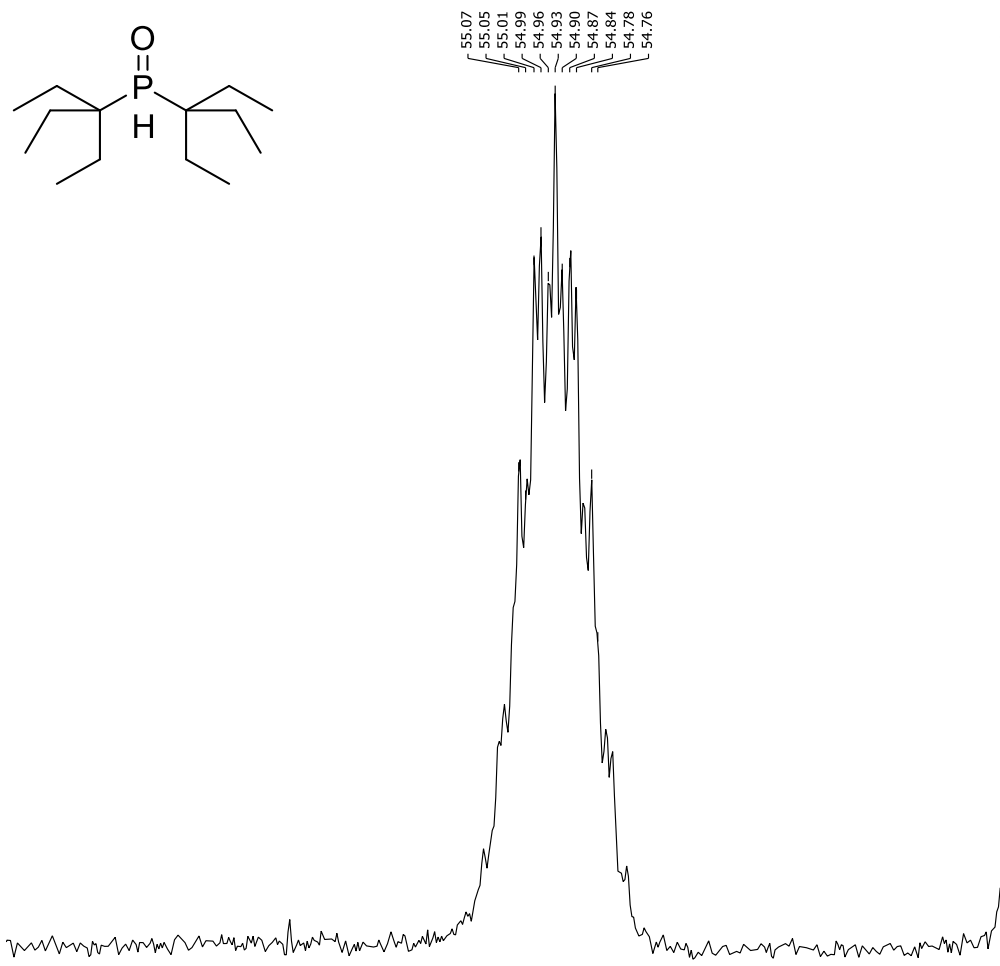

๙

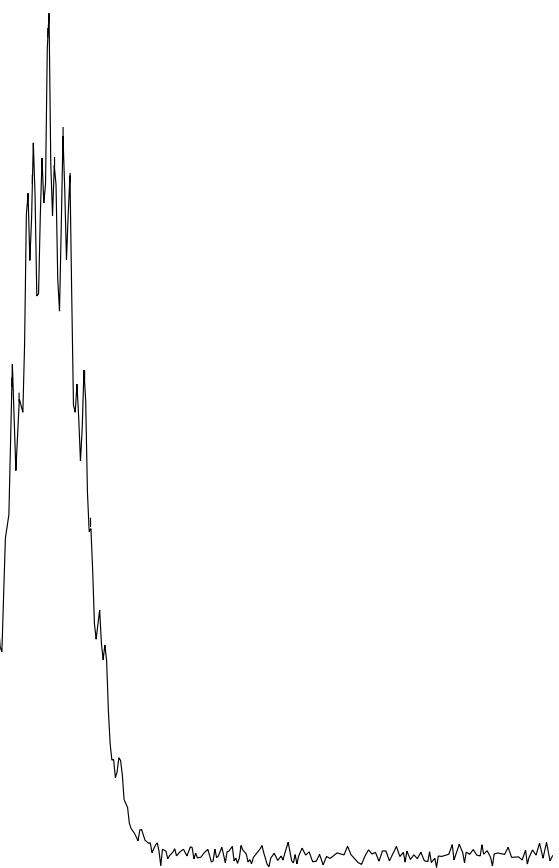


Di-(3-ethyl-3-pentyl)benzylphosphine borane complex - ${ }^{1} \mathrm{H}$ NMR (400 MHz, $\left.\mathrm{CDCl}_{3}\right)$
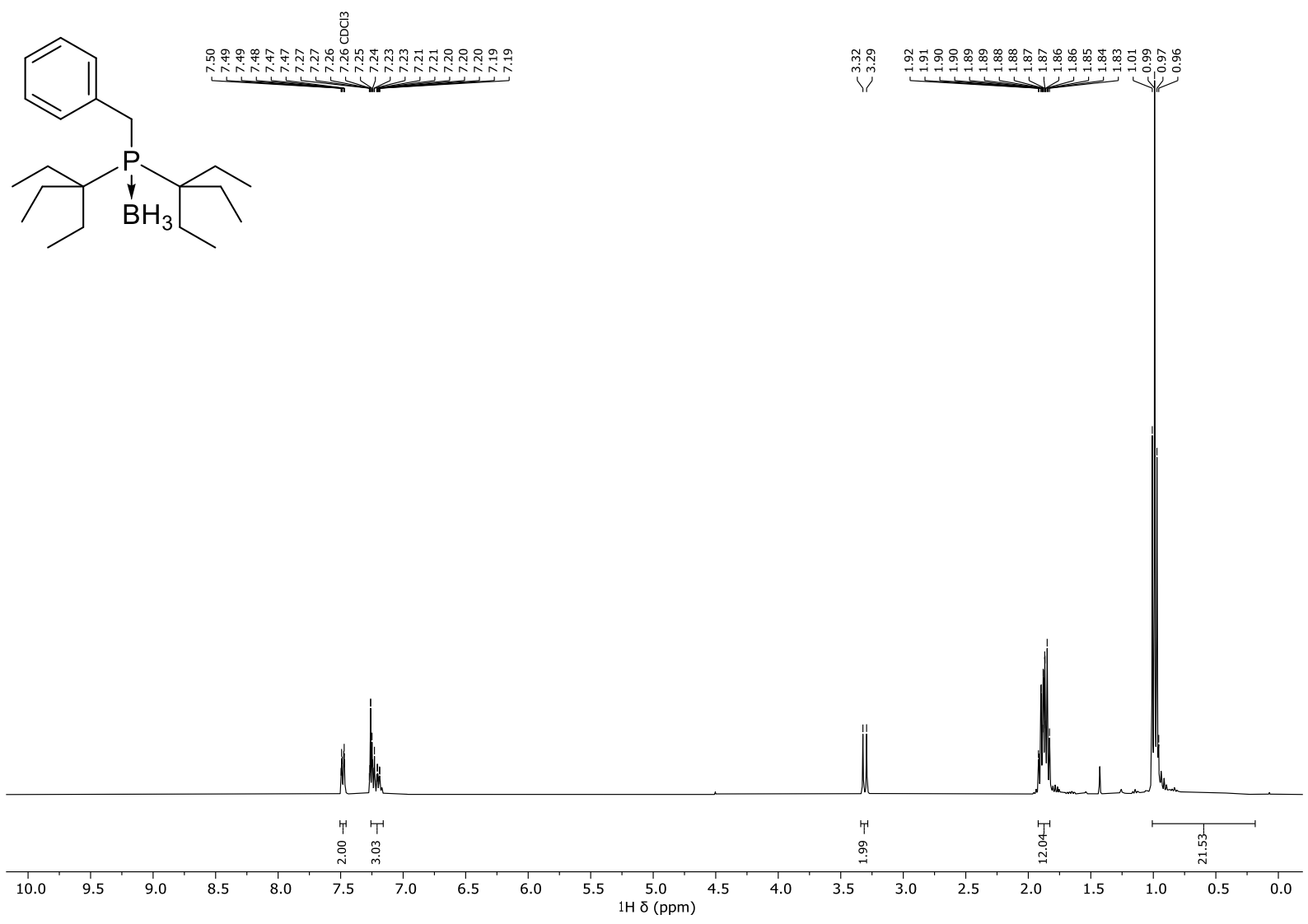

Di-(3-ethyl-3-pentyl)benzylphosphine borane complex ${ }^{11} \mathrm{~B}\left\{{ }^{1} \mathrm{H}\right\} \mathrm{NMR}\left(128 \mathrm{MHz}, \mathrm{CDCl}_{3}\right)$<smiles>[B]P(CC)(CC)(Cc1ccccc1)C(CC)(CC)CC</smiles> 
Di-(3-ethyl-3-pentyl)benzylphosphine borane complex $-{ }^{13} \mathrm{C}\left\{{ }^{1} \mathrm{H}\right\} \mathrm{NMR}\left(101 \mathrm{MHz}, \mathrm{CDCl}_{3}\right)$

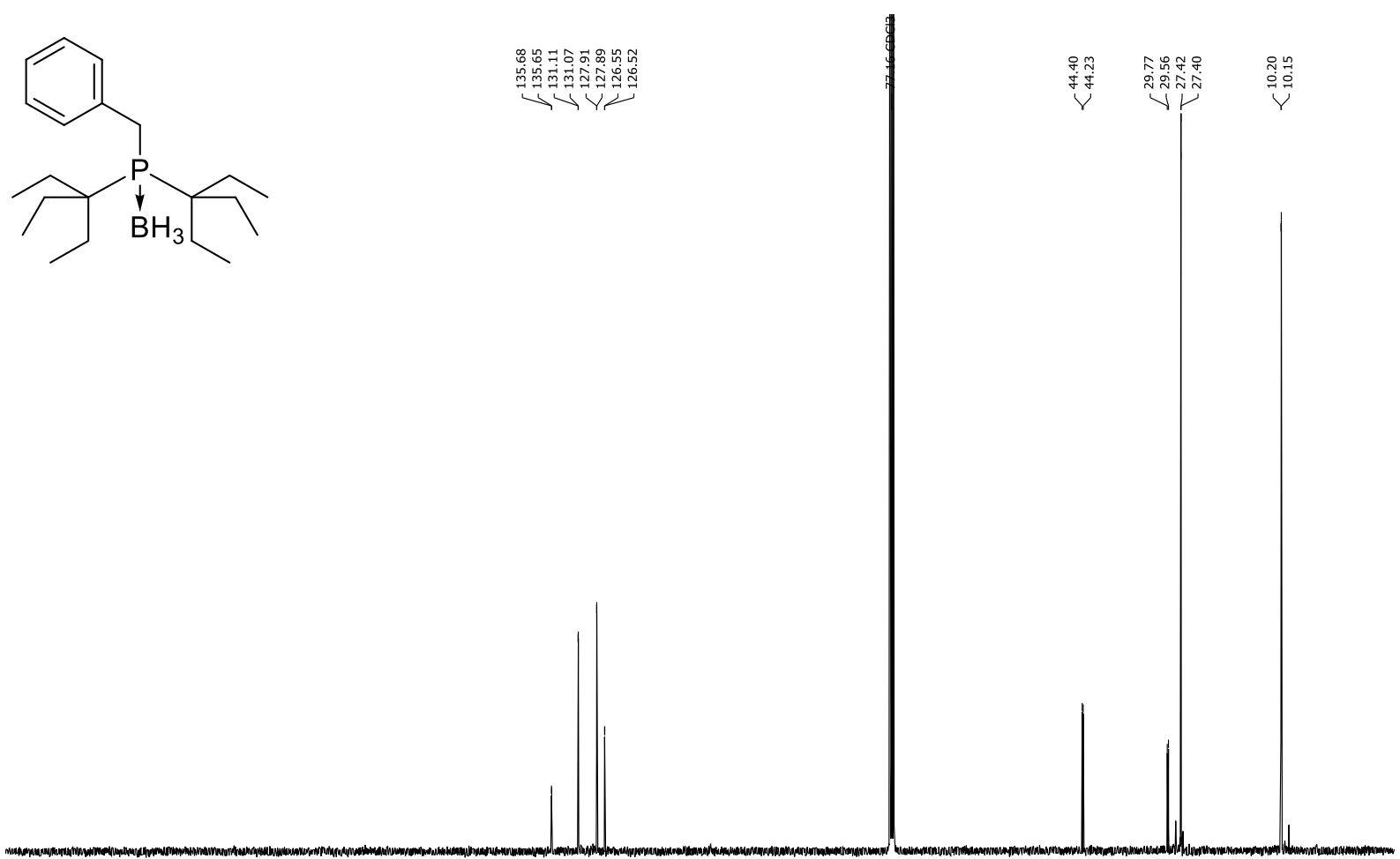

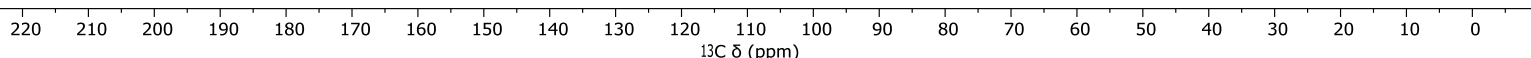

Di-(3-ethyl-3-pentyl)benzylphosphine borane complex ${ }^{31} \mathrm{P}\left\{{ }^{1} \mathrm{H}\right\} \mathrm{NMR}\left(162 \mathrm{MHz}, \mathrm{CDCl}_{3}\right)$<smiles>CCC(CC)[PH](Br)(Cc1ccccc1)C(CC)CC</smiles>

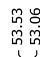

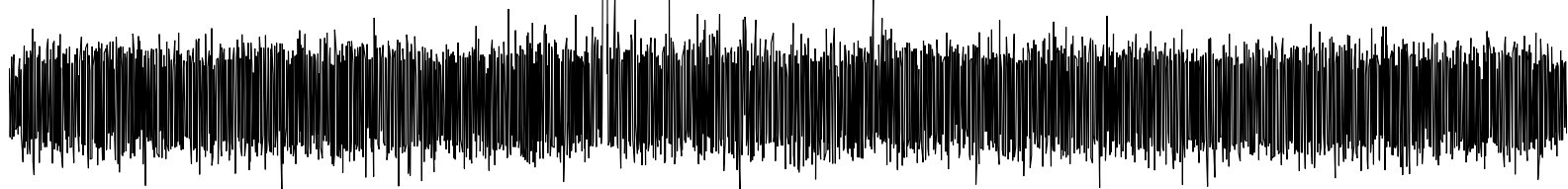

200

100

50 0
$31 \mathrm{P} \delta(\mathrm{ppm})$ 
2-Biphenyl triflate - ${ }^{1} \mathrm{H}$ NMR $\left(500 \mathrm{MHz}, \mathrm{CDCl}_{3}\right)$
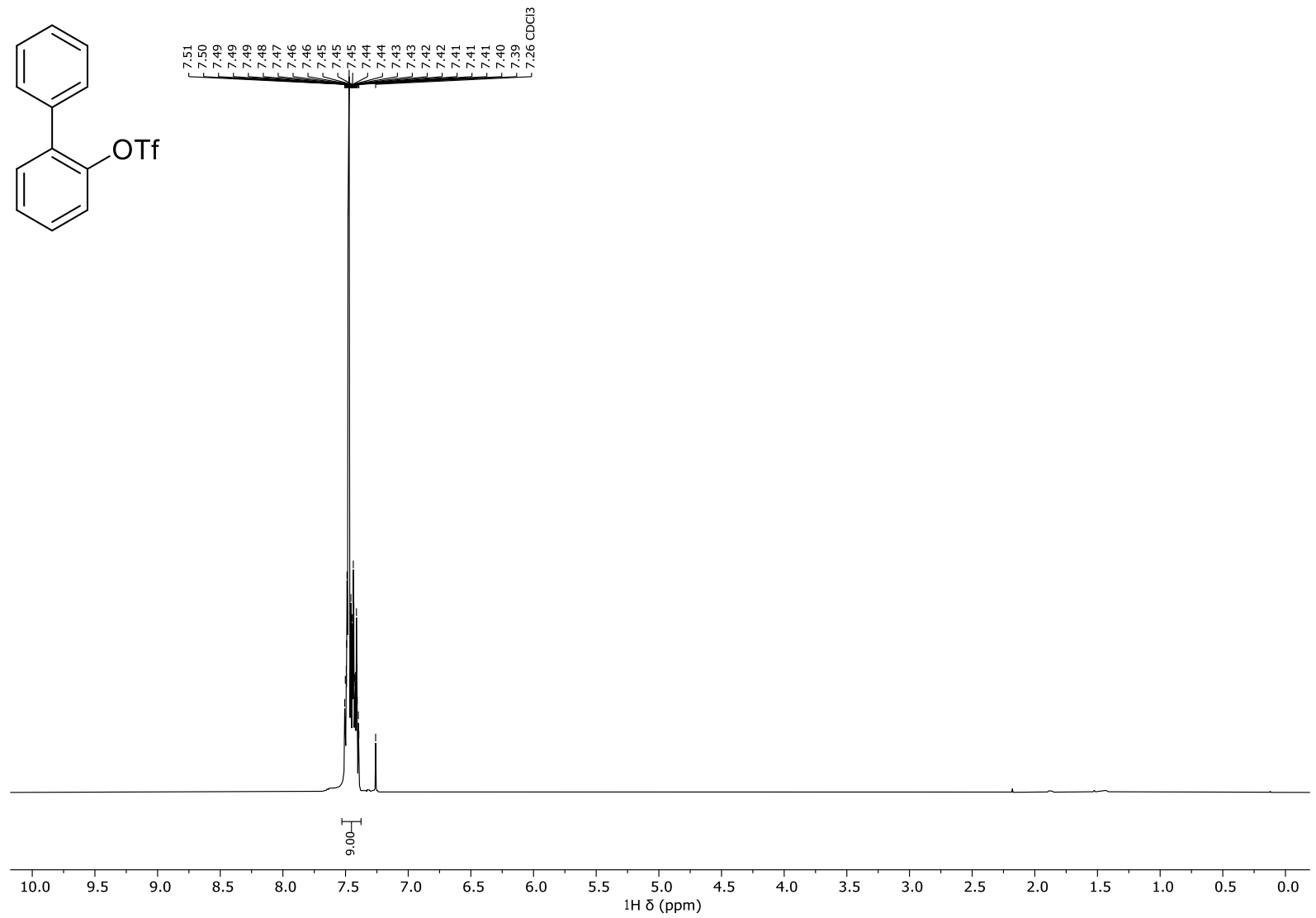

2-Biphenyl triflate $-{ }^{13} \mathrm{C}\left\{{ }^{1} \mathrm{H}\right\} \mathrm{NMR}\left(126 \mathrm{MHz}, \mathrm{CDCl}_{3}\right)$
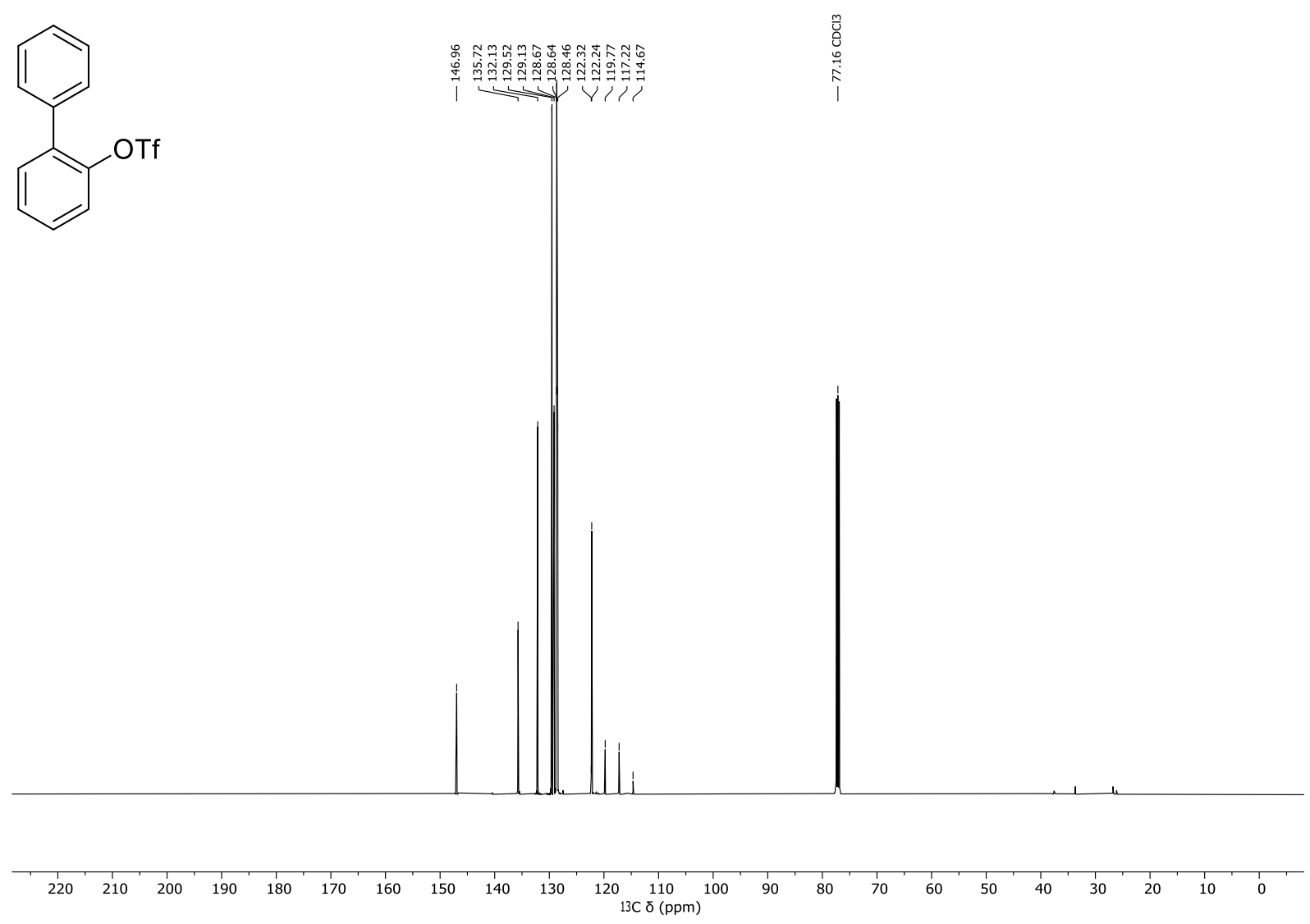

S169 
2-Biphenyl triflate ${ }^{19} \mathrm{~F}$ NMR $\left(470 \mathrm{MHz}, \mathrm{CDCl}_{3}\right)$

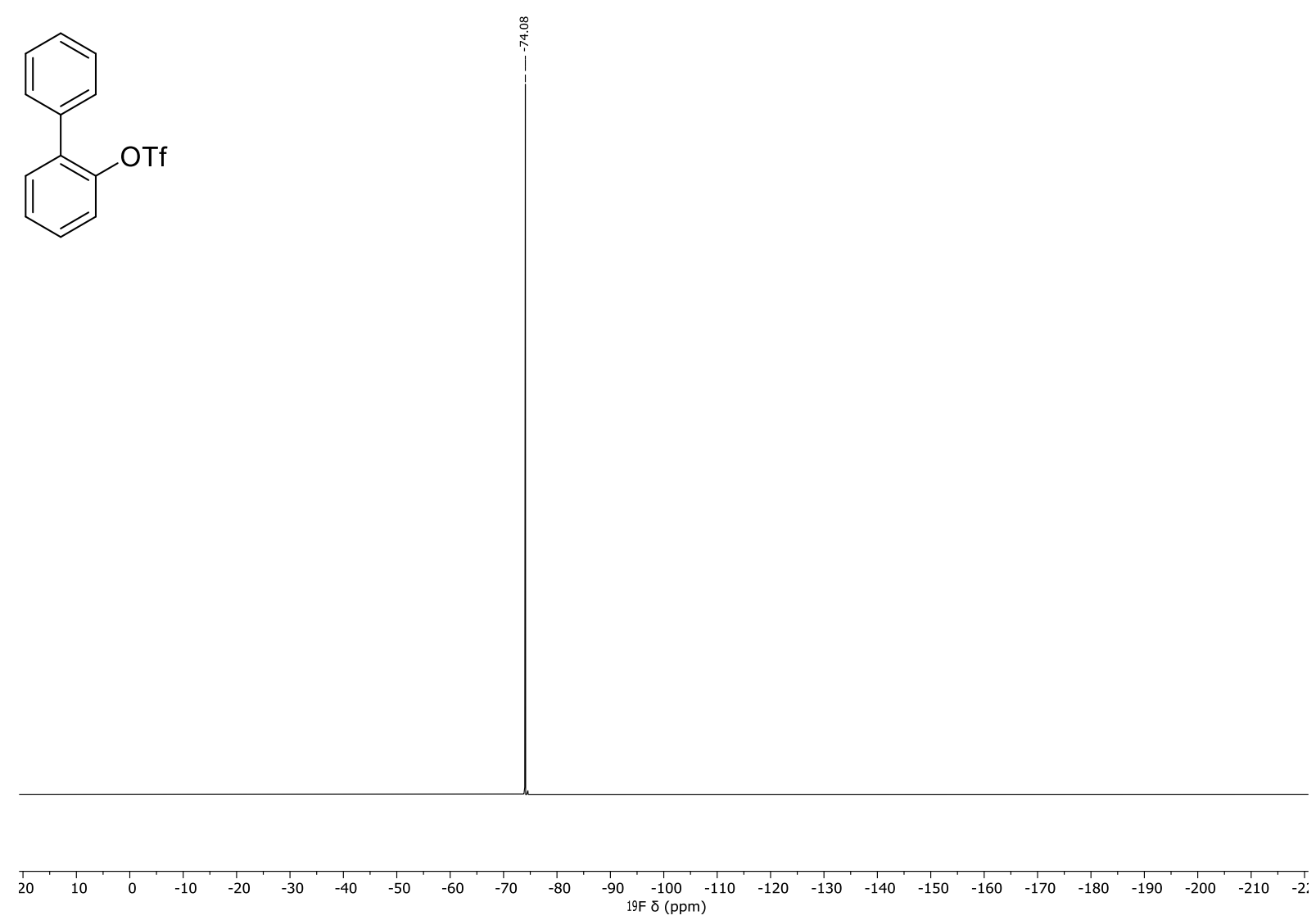


2-(Bis-(2,3-dimethyl-2-butyl)phosphino)biphenyl (3d) - $\left.{ }^{1} \mathrm{H} \mathrm{NMR} \mathrm{(500} \mathrm{MHz,} \mathrm{CDCl}_{3}\right)$

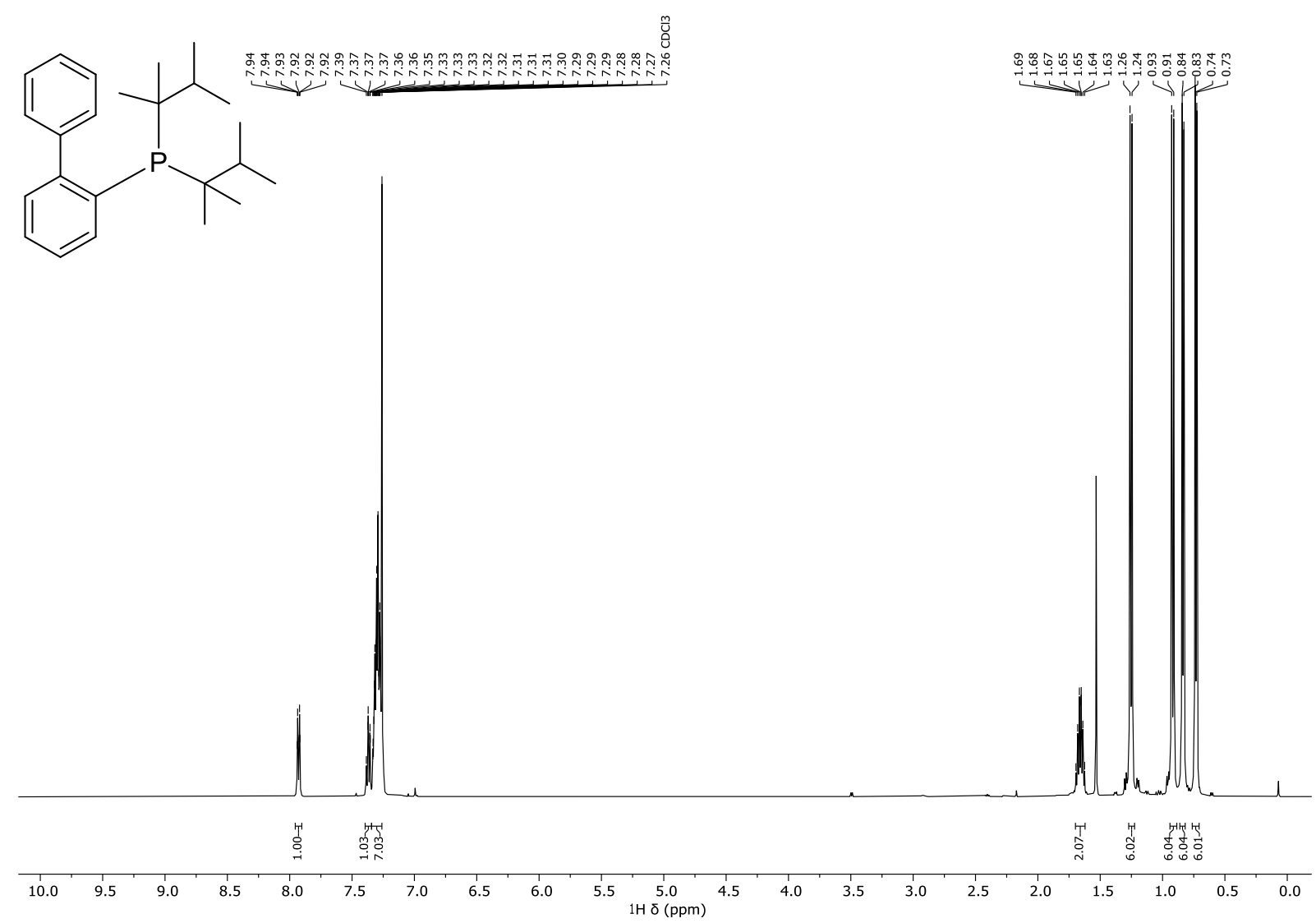

2-(Bis-(2,3-dimethyl-2-butyl)phosphino)biphenyl (3d) - ${ }^{13} \mathrm{C}\left\{{ }^{1} \mathrm{H}\right\} \mathrm{NMR}\left(126 \mathrm{MHz}, \mathrm{CDCl}_{3}\right)$<smiles>CC1CC(C)C(C)(C)P(c2ccccc2-c2ccccc2)C1(C)C</smiles>

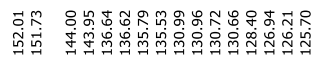

YY $\underset{Y}{ }$

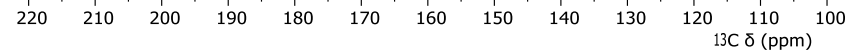


2-(Bis-(2,3-dimethyl-2-butyl)phosphino)biphenyl (3d) ${ }^{31} \mathrm{P}\left\{{ }^{1} \mathrm{H}\right\}$ NMR (202 $\left.\mathrm{MHz}, \mathrm{CDCl}_{3}\right)$

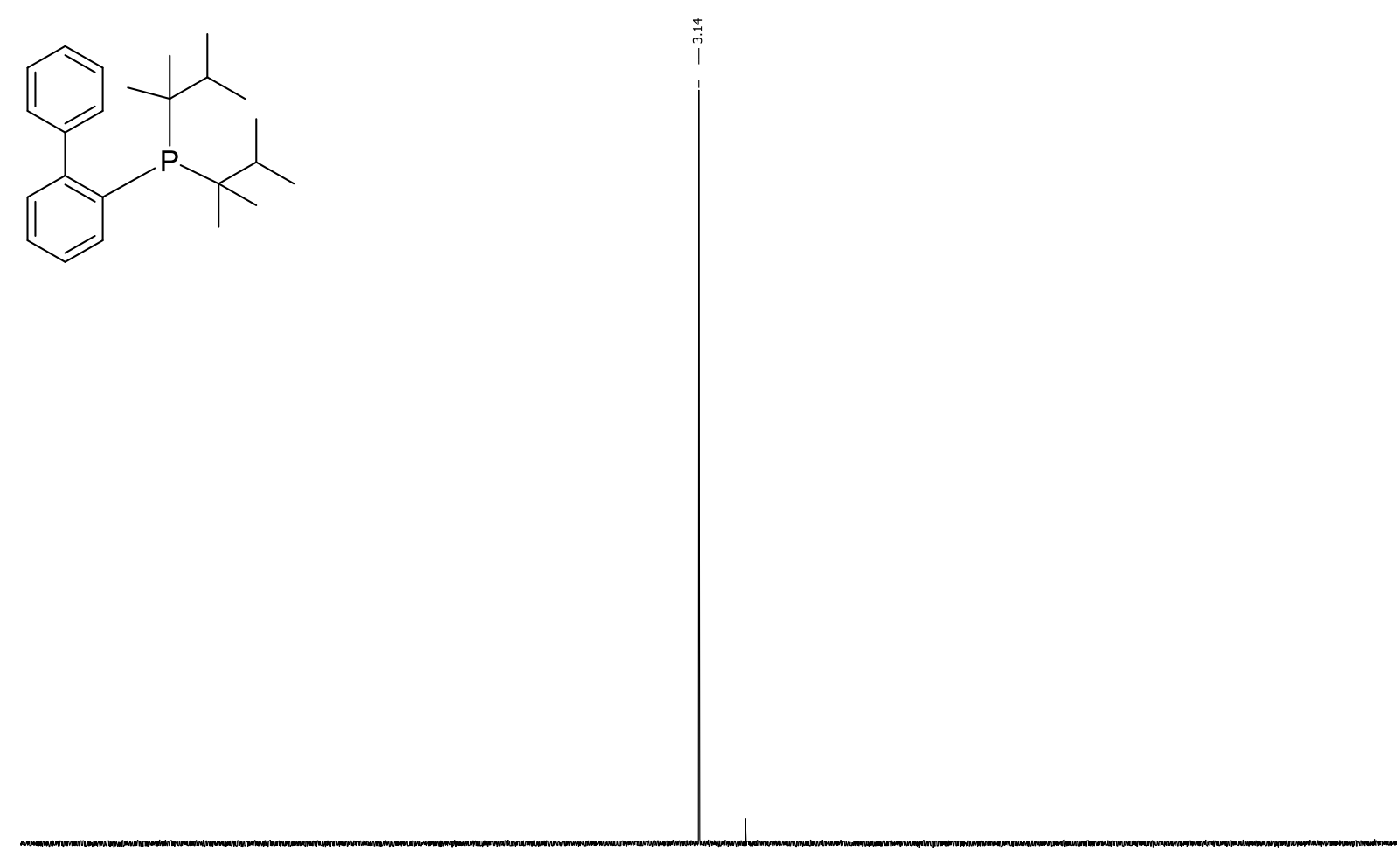

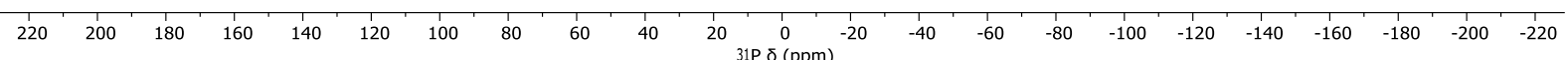




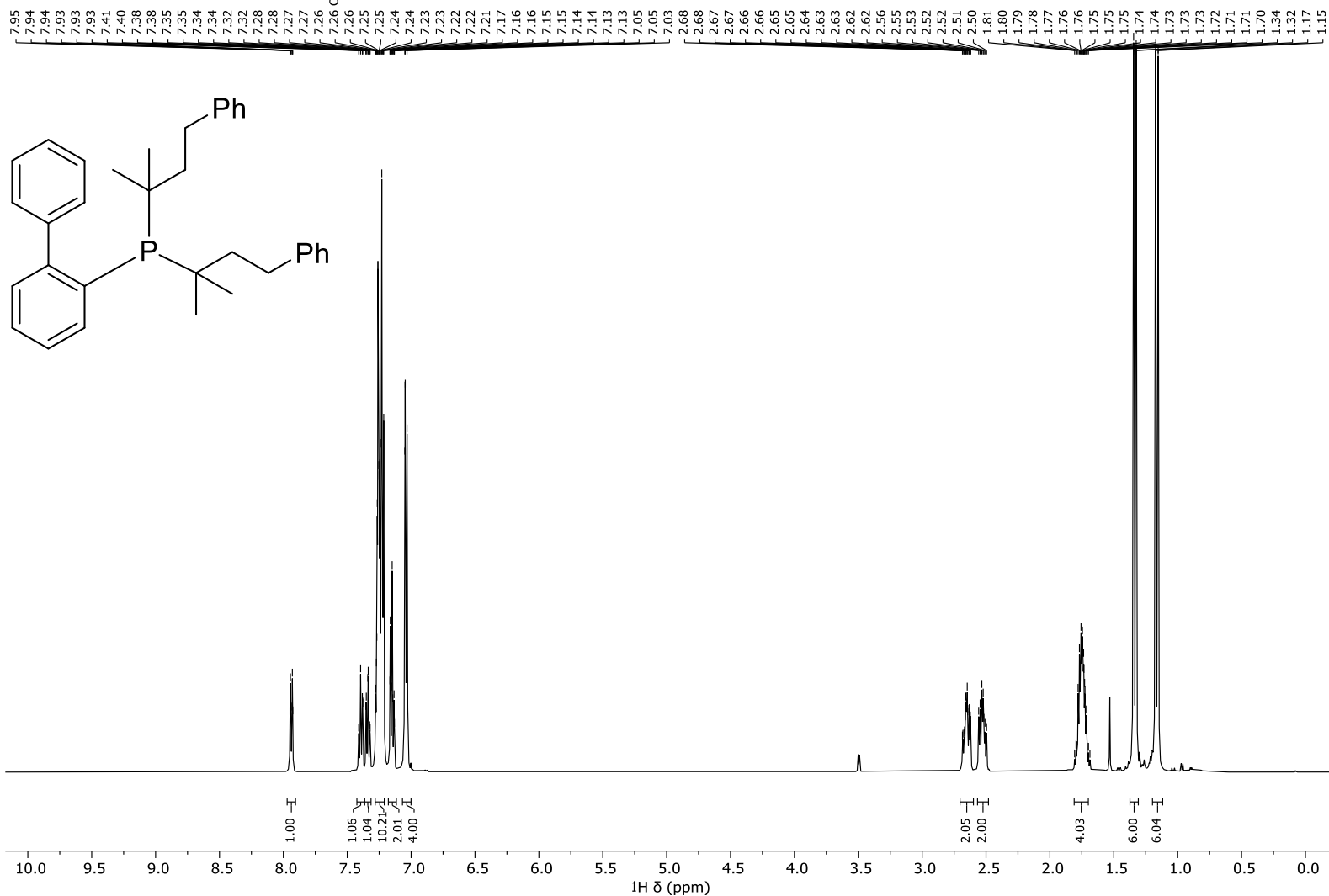

2-(Di-(2-methyl-4-phenyl-2-butyl)phosphino)biphenyl (3f) - ${ }^{13} \mathrm{C}\left\{{ }^{1} \mathrm{H}\right\} \mathrm{NMR}\left(126 \mathrm{MHz}, \mathrm{CDCl}_{3}\right)$<smiles>CC(C)(CCc1ccccc1)P(c1ccccc1)C(C)(C)CCc1ccccc1</smiles>

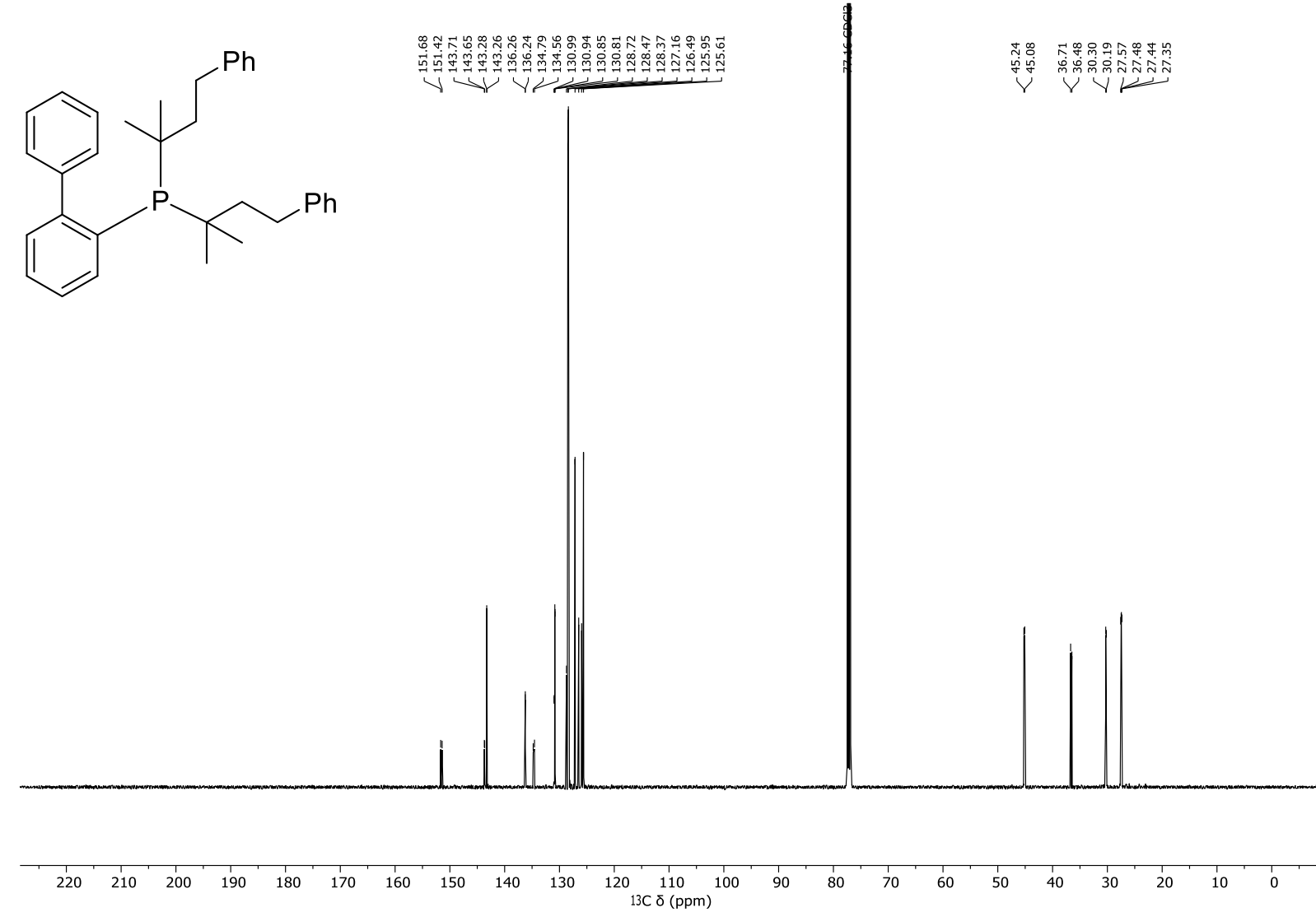

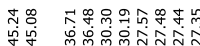

光 
2-(Di-(2-methyl-4-phenyl-2-butyl)phosphino)biphenyl (3f) ${ }^{31} \mathrm{P}\left\{{ }^{1} \mathrm{H}\right\} \mathrm{NMR}\left(202 \mathrm{MHz}, \mathrm{CDCl}_{3}\right)$<smiles>CC(C)(CCc1ccccc1)P(c1ccccc1)C(C)(C)CCc1ccccc1</smiles> 
2-(Di-(1-methylcyclopentyl)phosphino)biphenyl (3h) - ${ }^{1} \mathrm{H}$ NMR (500 MHz, $\mathrm{CDCl}_{3}$ )

8

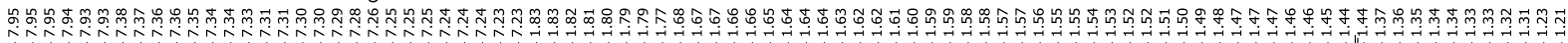<smiles>CC1(P(c2ccccc2-c2ccccc2)C2(C)CCCC2)CCCC1</smiles>

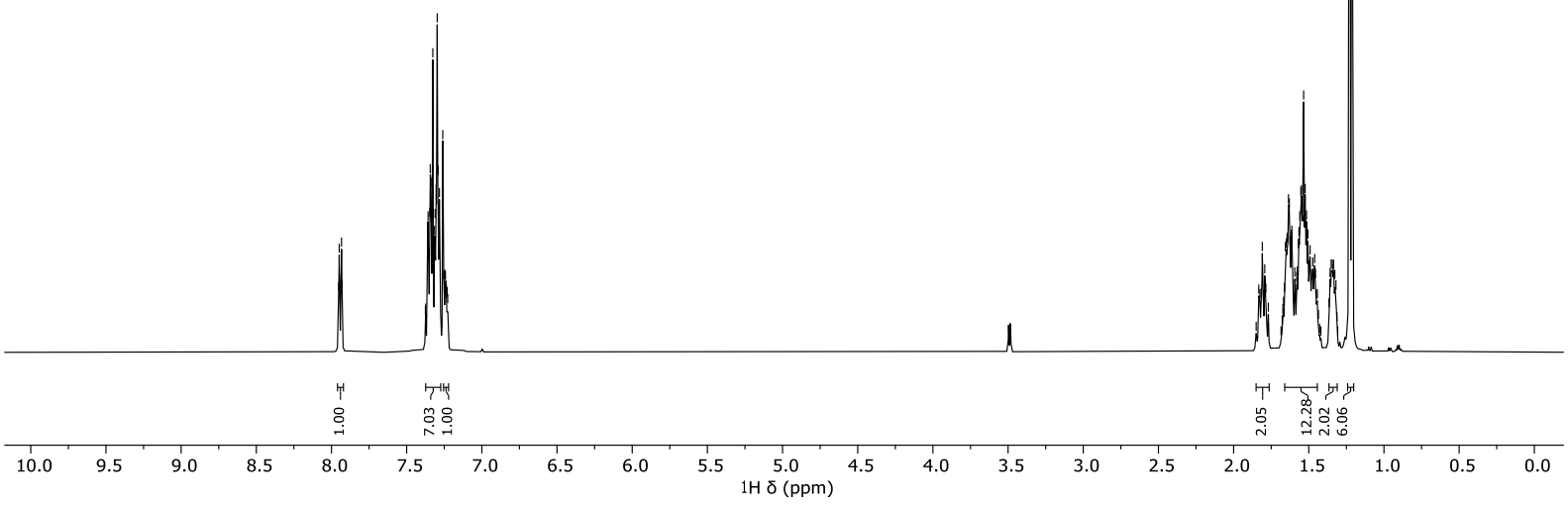

2-(Di-(1-methylcyclopentyl)phosphino)biphenyl (3h) - ${ }^{13} \mathrm{C}\left\{{ }^{1} \mathrm{H}\right\} \mathrm{NMR}\left(126 \mathrm{MHz}, \mathrm{CDCl}_{3}\right)$<smiles>CC1(P(c2ccccc2)c2ccccc2)CCCC1</smiles>

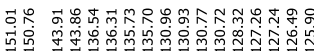

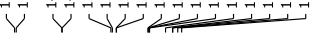

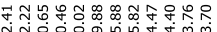

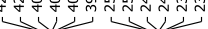

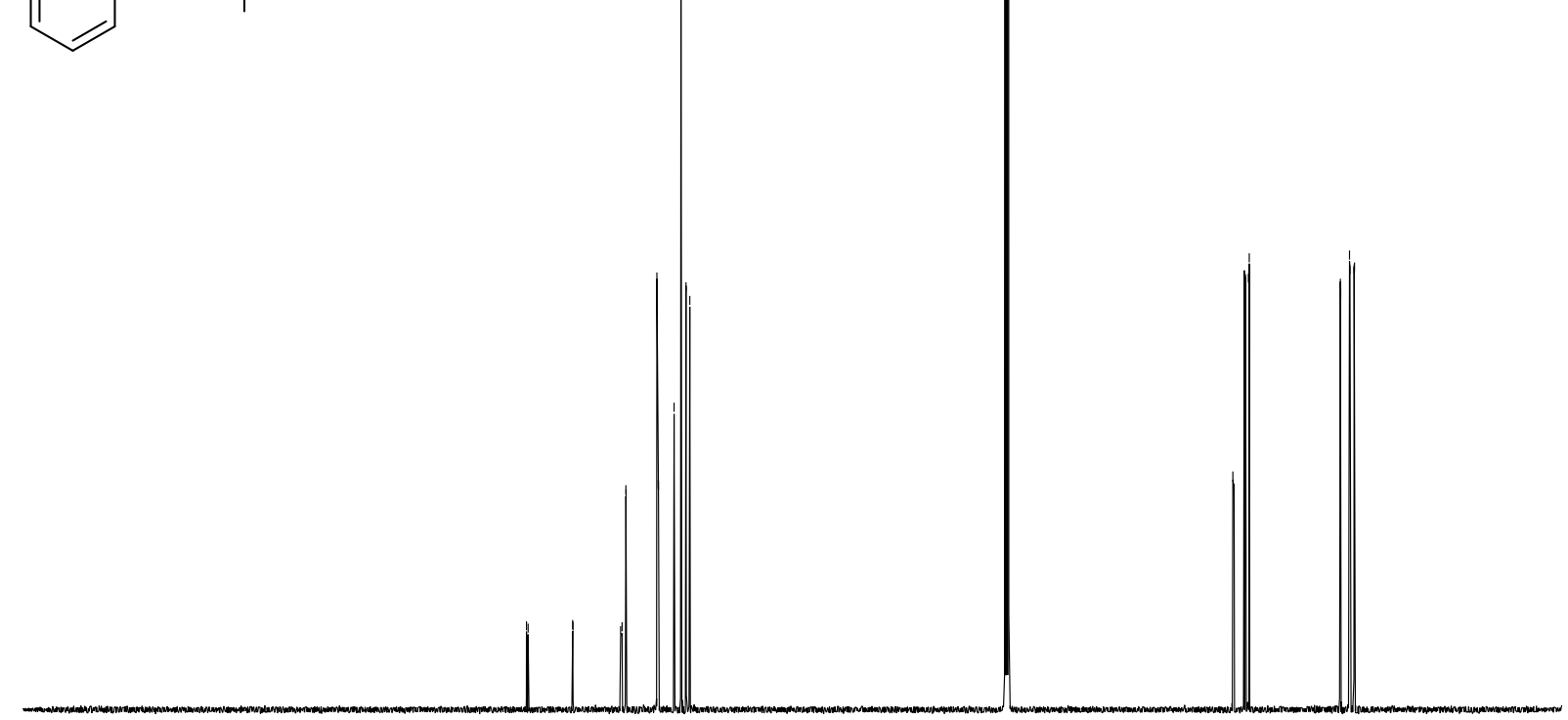

$\begin{array}{lllllllllll}220 & 210 & 200 & 190 & 180 & 170 & 160 & 150 & 140 & 130 & 120 \\ 13 \mathrm{C} \delta(\mathrm{ppm}) & 100\end{array}$ 
2-(Di-(1-methylcyclopentyl)phosphino)biphenyl (3h) ${ }^{31} \mathrm{P}\left\{{ }^{1} \mathrm{H}\right\}$ NMR (202 MHz, $\left.\mathrm{CDCl}_{3}\right)$<smiles>CC1(c2ccccc2P(c2ccccc2)C2(C)CCCC2)CCCC1</smiles>

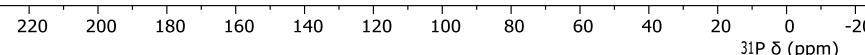


2-(Di-(1-methylcyclohexyl)phosphino)biphenyl (3i) - ${ }^{1} \mathrm{H} \mathrm{NMR}\left(500 \mathrm{MHz}, \mathrm{CDCl}_{3}\right)$
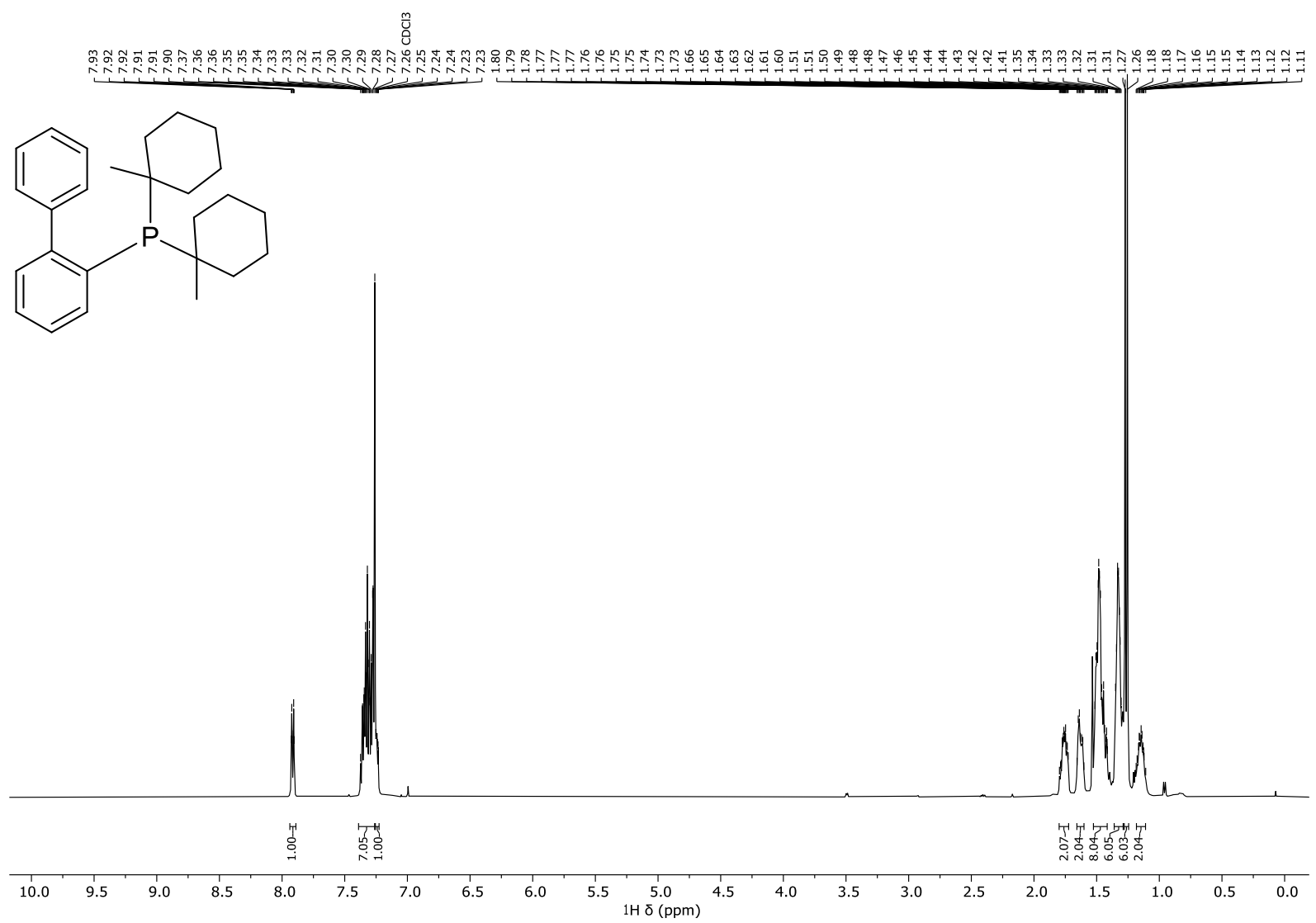

2-(Di-(1-methylcyclohexyl)phosphino)biphenyl (3i) - ${ }^{13} \mathrm{C}\left\{{ }^{1} \mathrm{H}\right\}$ NMR (126 MHz, CDCl 3 )
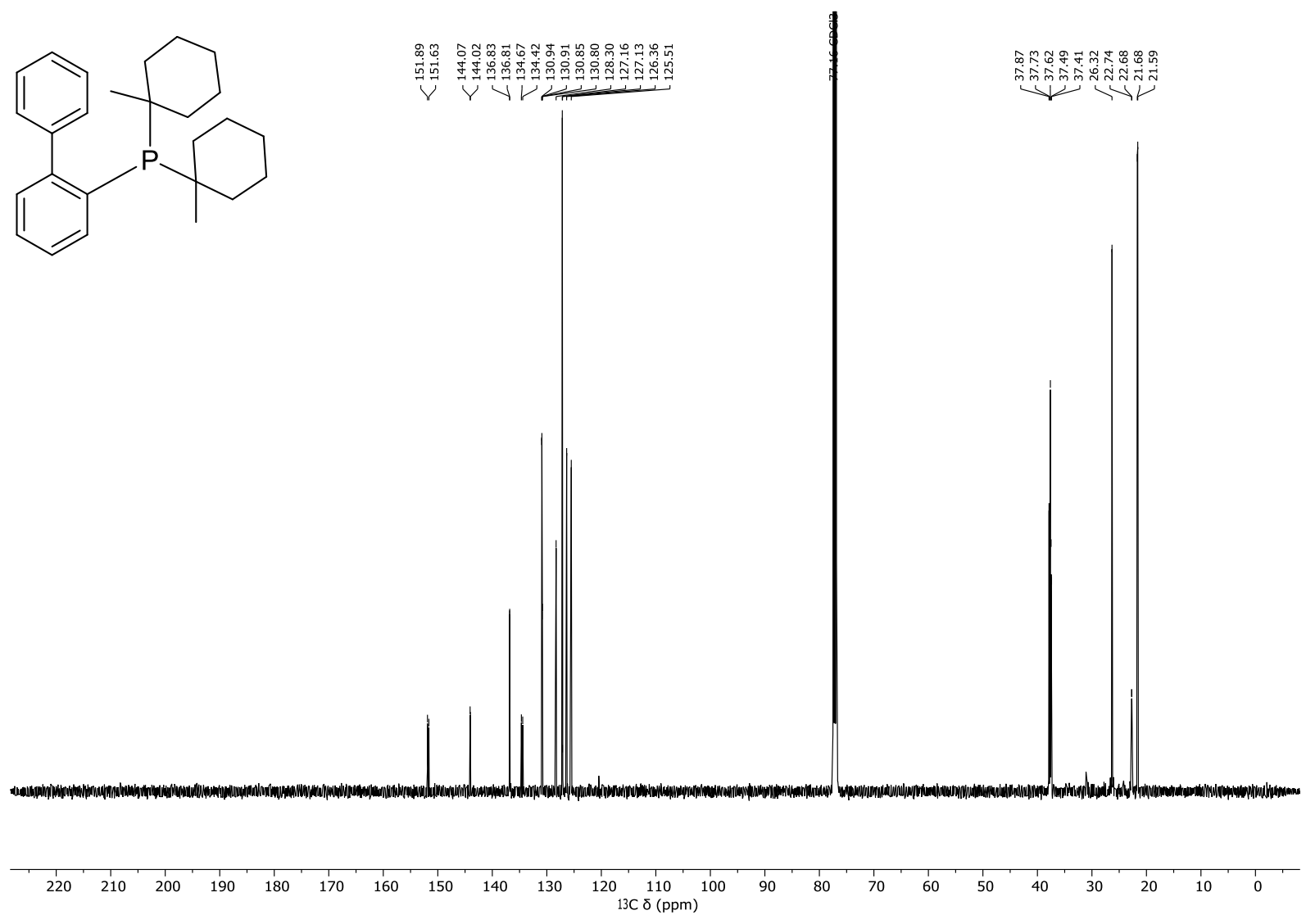

S177 
2-(Di-(1-methylcyclohexyl)phosphino)biphenyl (3i) + 2\% XPhos $\left(\delta_{\mathrm{p}}-12.31 \mathrm{ppm}\right){ }^{31} \mathrm{P}\left\{{ }^{1} \mathrm{H}\right\}$ NMR (202 $\mathrm{MHz}, \mathrm{CDCl}_{3}$ )

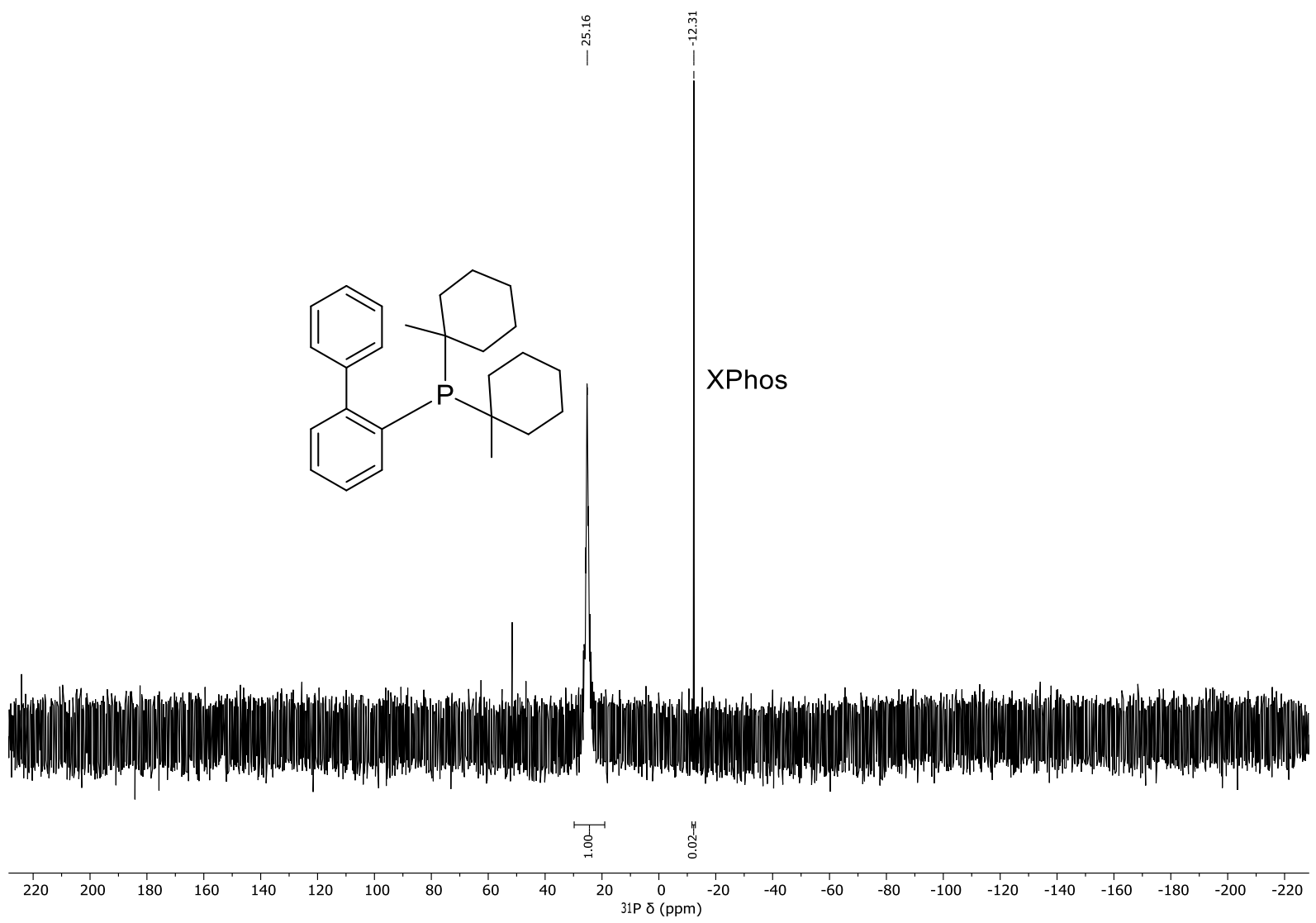


2-(Di-(1-adamantyl)phosphino)biphenyl (3k) - $\left.{ }^{1} \mathrm{H} \mathrm{NMR} \mathrm{(500} \mathrm{MHz,} \mathrm{CDCl}_{3}\right)$
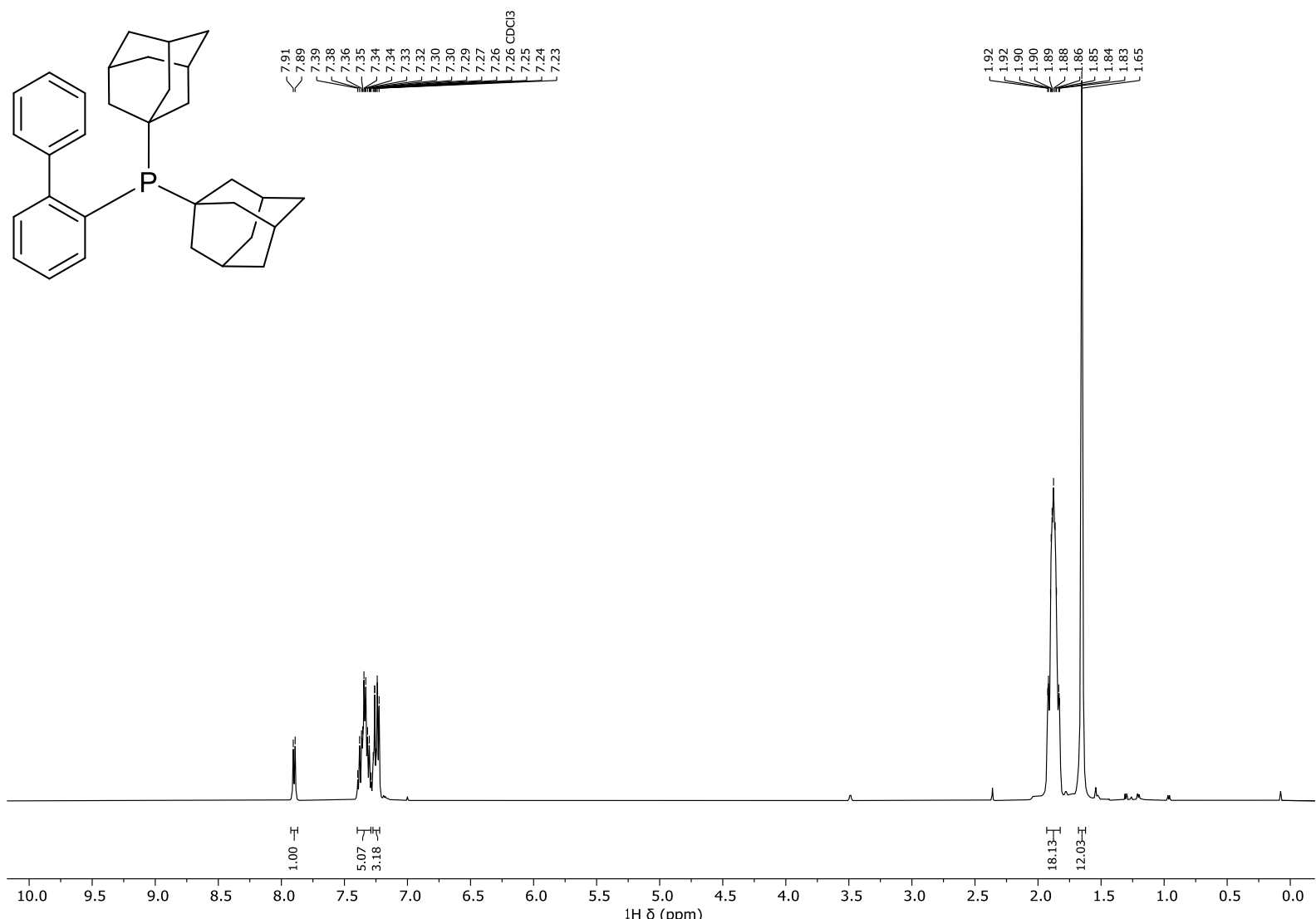

2-(Di-(1-adamantyl)phosphino)biphenyl (3k) - ${ }^{13} \mathrm{C}\left\{{ }^{1} \mathrm{H}\right\}$ NMR (126 MHz, $\mathrm{CDCl}_{3}$ )<smiles>c1ccc(-c2ccccc2P(C23CC4CC(CC(C4)C2)C3)C23CC4CC(CC(C4)C2)C3)cc1</smiles>

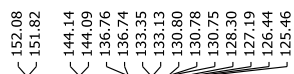

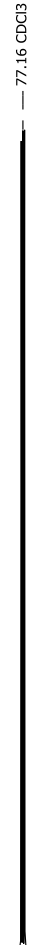

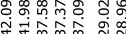

y

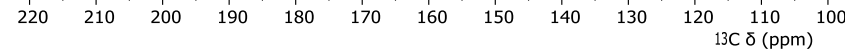


2-(Di-(1-adamantyl)phosphino)biphenyl (3k) ${ }^{31} \mathrm{P}\left\{{ }^{1} \mathrm{H}\right\} \mathrm{NMR}\left(202 \mathrm{MHz}, \mathrm{CDCl}_{3}\right)$<smiles>c1ccc(-c2ccccc2P(C23CC4CC(CC(C4)C2)C3)C23CC4CC(CC(C4)C2)C3)cc1</smiles> 
2-(Di-(2-methyl-2-adamantyl)phosphino)biphenyl (3l) - ${ }^{1} \mathrm{H}$ NMR $\left(500 \mathrm{MHz}, \mathrm{CDCl}_{3}\right)$

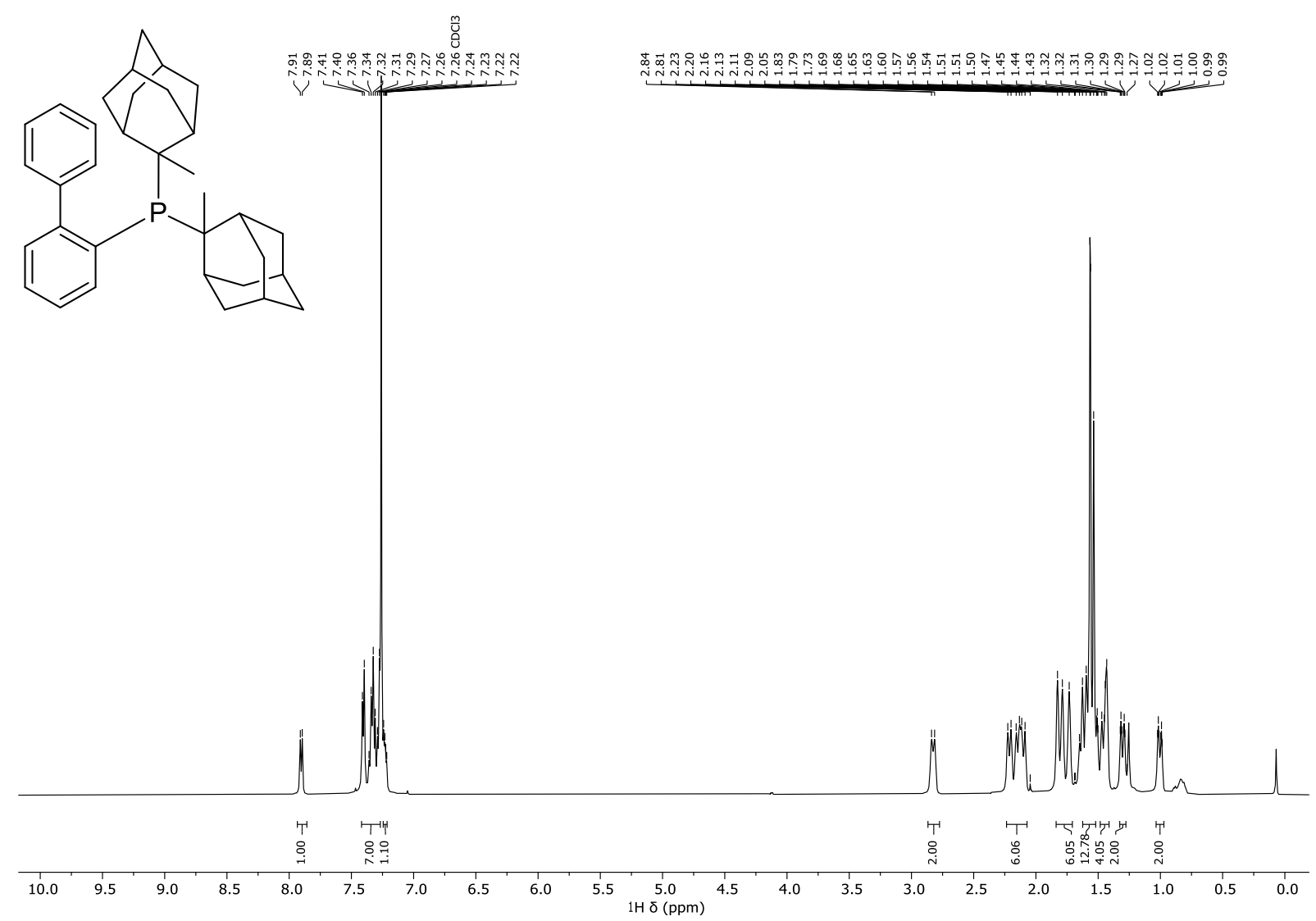

2-(Di-(2-methyl-2-adamantyl)phosphino)biphenyl (3I) - ${ }^{13} \mathrm{C}\left\{{ }^{1} \mathrm{H}\right\}$ NMR $\left(126 \mathrm{MHz}, \mathrm{CDCl}_{3}\right)$

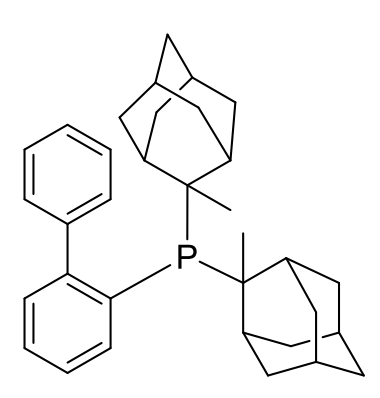

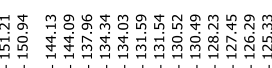

P Y iv

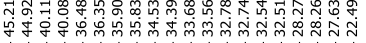

$\begin{array}{lllllllllllll}220 & 210 & 200 & 190 & 180 & 170 & 160 & 150 & 140 & 130 & 120 & 110 & 100 \\ 13 \mathrm{C} \delta(\mathrm{ppm})\end{array}$ 
2-(Di-(2-methyl-2-adamantyl)phosphino)biphenyl (3l) ${ }^{31} \mathrm{P}\left\{{ }^{1} \mathrm{H}\right\} \mathrm{NMR}\left(202 \mathrm{MHz}, \mathrm{CDCl}_{3}\right)$<smiles>CC(C)(P(c1ccccc1-c1ccccc1)C12CC3CC(CC(C3)C1)C2)C12CC3CC(CC(C3)C1)C2</smiles>

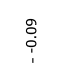

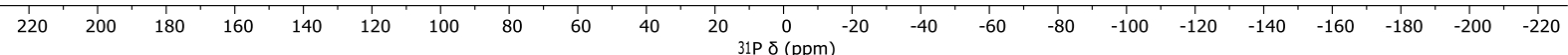


Crude 2-(di-(tert-amyl)phosphino)biphenyl (3a) ${ }^{31} \mathrm{P}\left\{{ }^{1} \mathrm{H}\right\}$ NMR (202 MHz, $\left.\mathrm{CDCl}_{3}\right)$
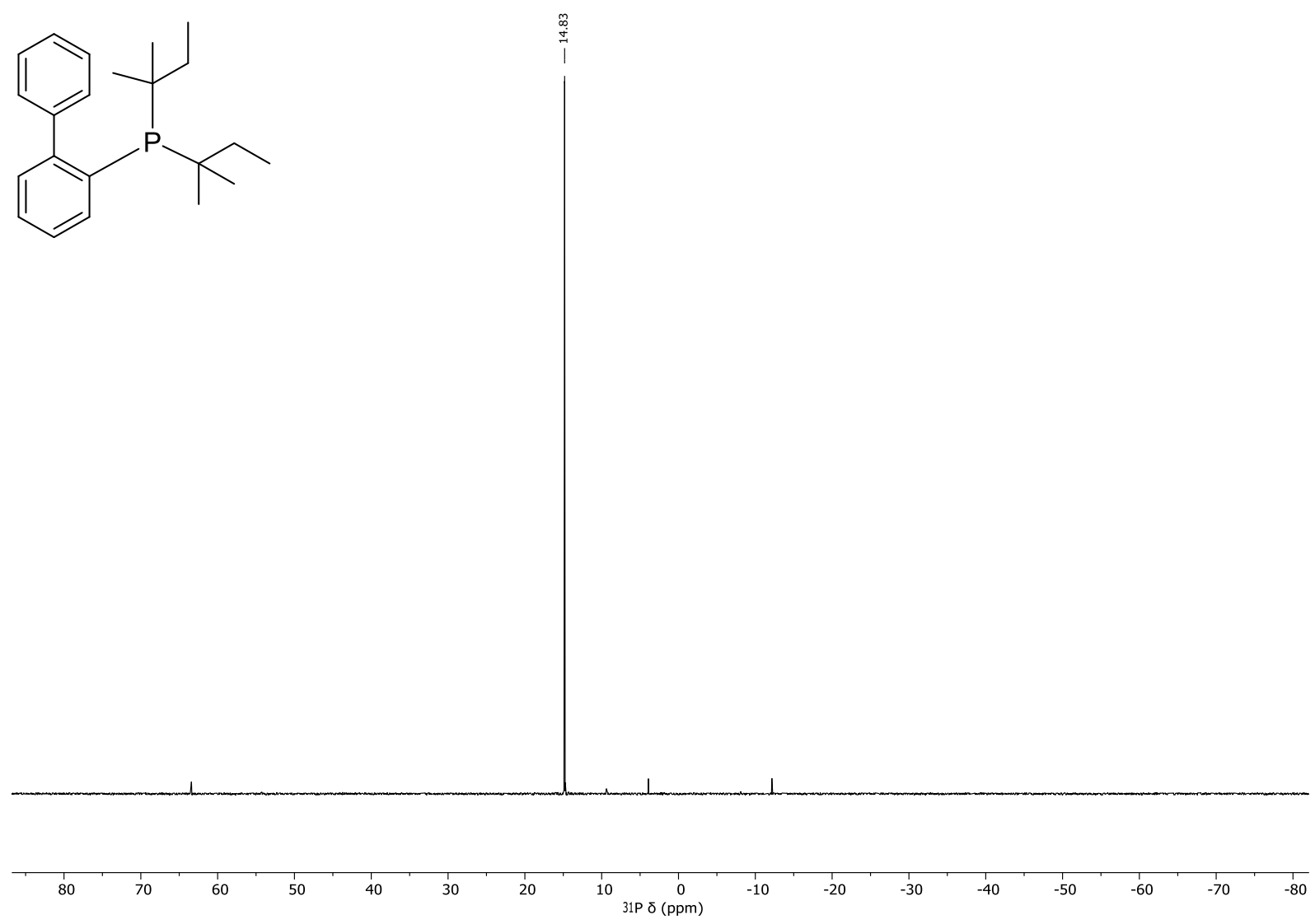

(2-Biphenyl)di-tert-amylphosphine selenide (4a) reaction mixture ${ }^{31} \mathrm{P}\left\{{ }^{1} \mathrm{H}\right\} \mathrm{NMR}\left(202 \mathrm{MHz}, \mathrm{CDCl}_{3}\right)$<smiles>[R6]c1ccccc1-c1ccccc1</smiles>
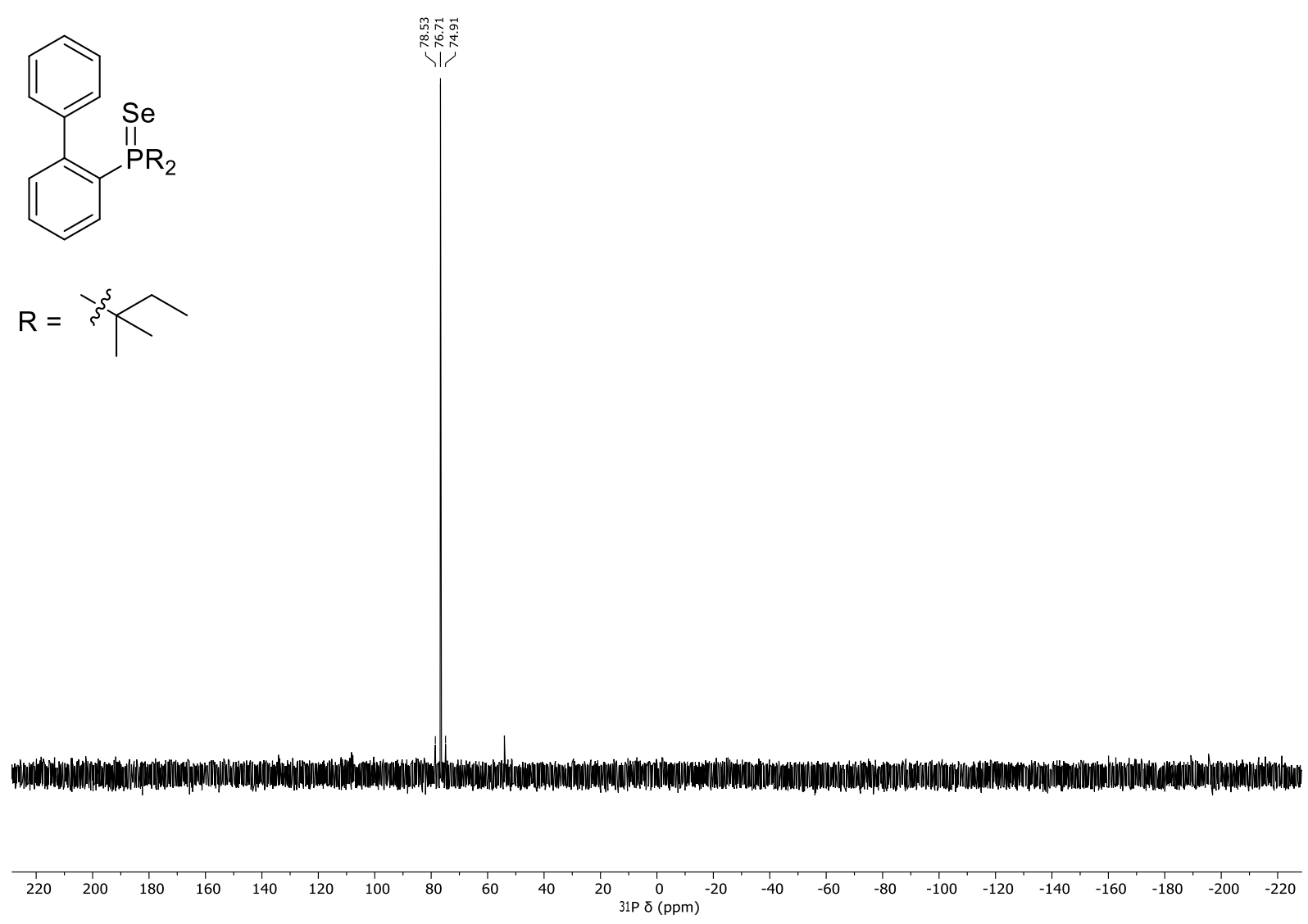

S183 
Crude 2-(Di-(3-methyl-3-pentyl)phosphino)biphenyl (3b) ${ }^{31} \mathrm{P}\left\{{ }^{1} \mathrm{H}\right\} \mathrm{NMR}\left(202 \mathrm{MHz}, \mathrm{CDCl}_{3}\right)$<smiles>CCC(CC)(CC)P(c1ccccc1-c1ccccc1)C(CC)(CC)CC</smiles>

(2-Biphenyl)di-(3-methyl-3-pentyl)phosphine selenide (4b) reaction mixture ${ }^{31} \mathrm{P}\left\{{ }^{1} \mathrm{H}\right\} \mathrm{NMR}\left(202 \mathrm{MHz}, \mathrm{CDCl}_{3}\right)$<smiles>[R][Y5](=[R5])c1ccccc1-c1ccccc1</smiles>

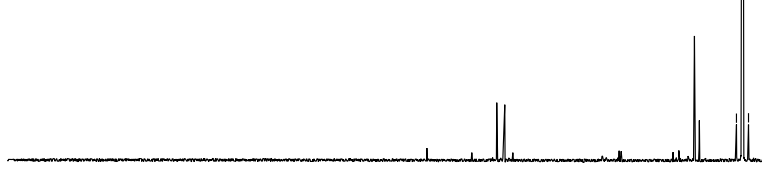


Crude 2-(Di-(3-ethyl-3-pentyl)phosphino)biphenyl (3c) ${ }^{31} \mathrm{P}\left\{{ }^{1} \mathrm{H}\right\}$ NMR (202 $\left.\mathrm{MHz}, \mathrm{CDCl}_{3}\right)$<smiles>CCC(CC)(CC)P(c1ccccc1-c1ccccc1)C(CC)(CC)CC</smiles>

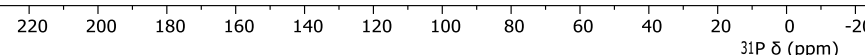

(2-Biphenyl)di-(3-ethyl-3-pentyl)phosphine selenide (4c) reaction mixture $\left.{ }^{31} \mathrm{P}^{1}{ }^{1} \mathrm{H}\right\} \mathrm{NMR}\left(202 \mathrm{MHz}, \mathrm{CDCl}_{3}\right)$<smiles>[R][R15](=[Se])c1ccccc1-c1ccccc1</smiles> 
(2-Biphenyl)bis-(2,3-dimethyl-2-butyl)phosphine selenide (4d) reaction mixture ${ }^{31} \mathrm{P}\left\{{ }^{1} \mathrm{H}\right\}$ NMR (202 $\left.\mathrm{MHz}, \mathrm{CDCl}_{3}\right)$<smiles>[R][Y](=[R16])(=S)c1ccccc1-c1ccccc1</smiles>
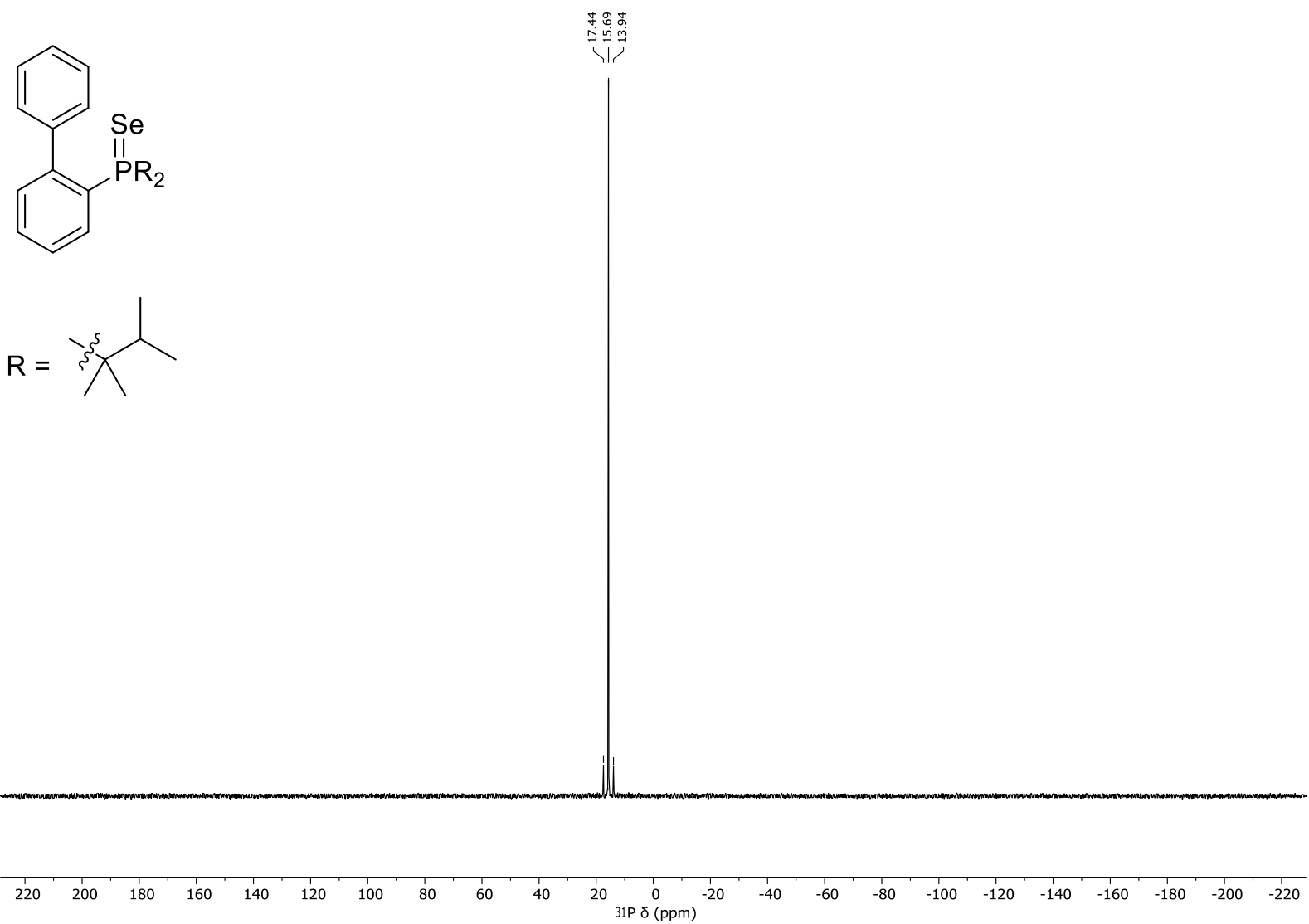
(2-Biphenyl)di-(2-methyl-4-phenyl-2-butyl)phosphine selenide (4f) reaction mixture ${ }^{31} \mathrm{P}\left\{{ }^{1} \mathrm{H}\right\} \mathrm{NMR}$ (202 $\mathrm{MHz}, \mathrm{CDCl}_{3}$ )<smiles>[R][Y]([H])(C)C(C)(C)CCc1ccccc1</smiles>

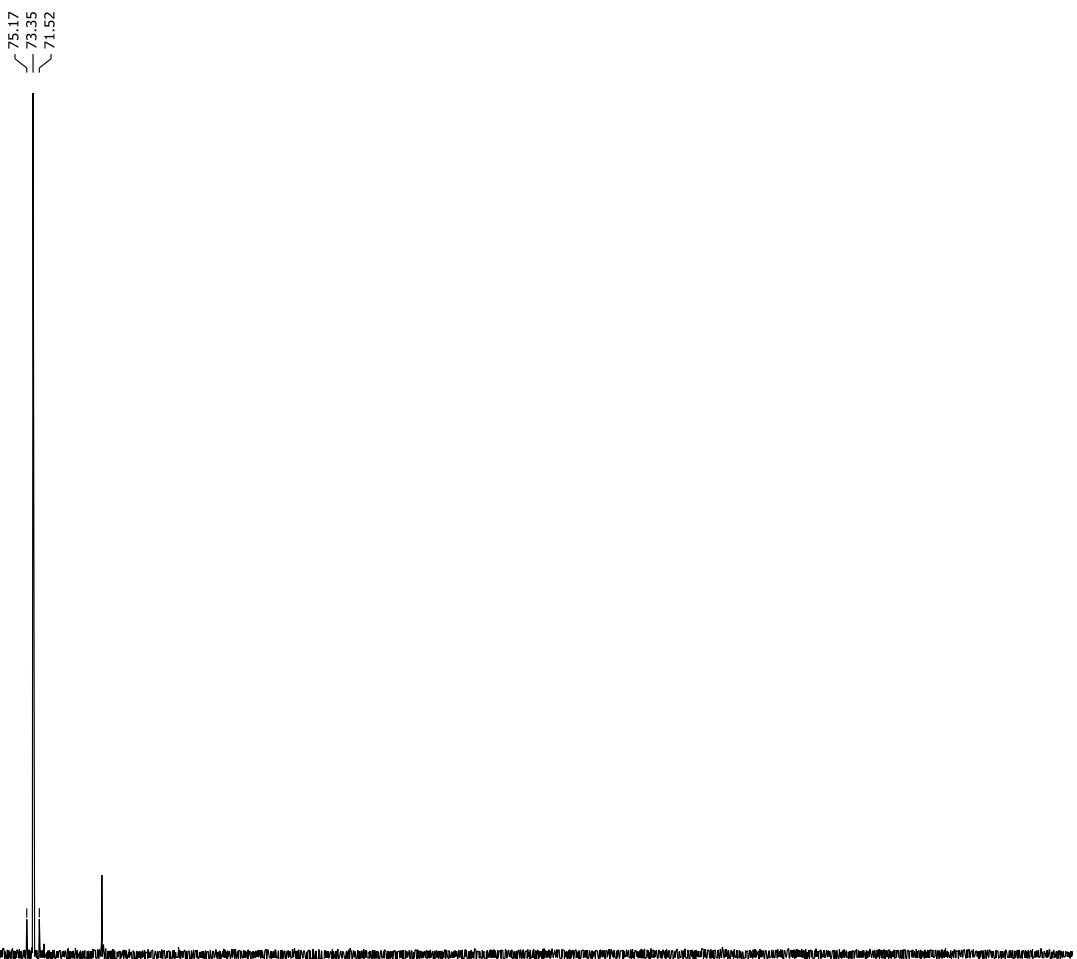

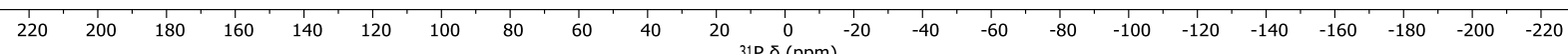


(2-Biphenyl)di-(1-methylcyclopentyl)phosphine selenide $(4 \mathrm{~h})$ reaction mixture ${ }^{31} \mathrm{P}\left\{{ }^{1} \mathrm{H}\right\}$ NMR $(202$ $\mathrm{MHz}, \mathrm{CDCl}_{3}$ )
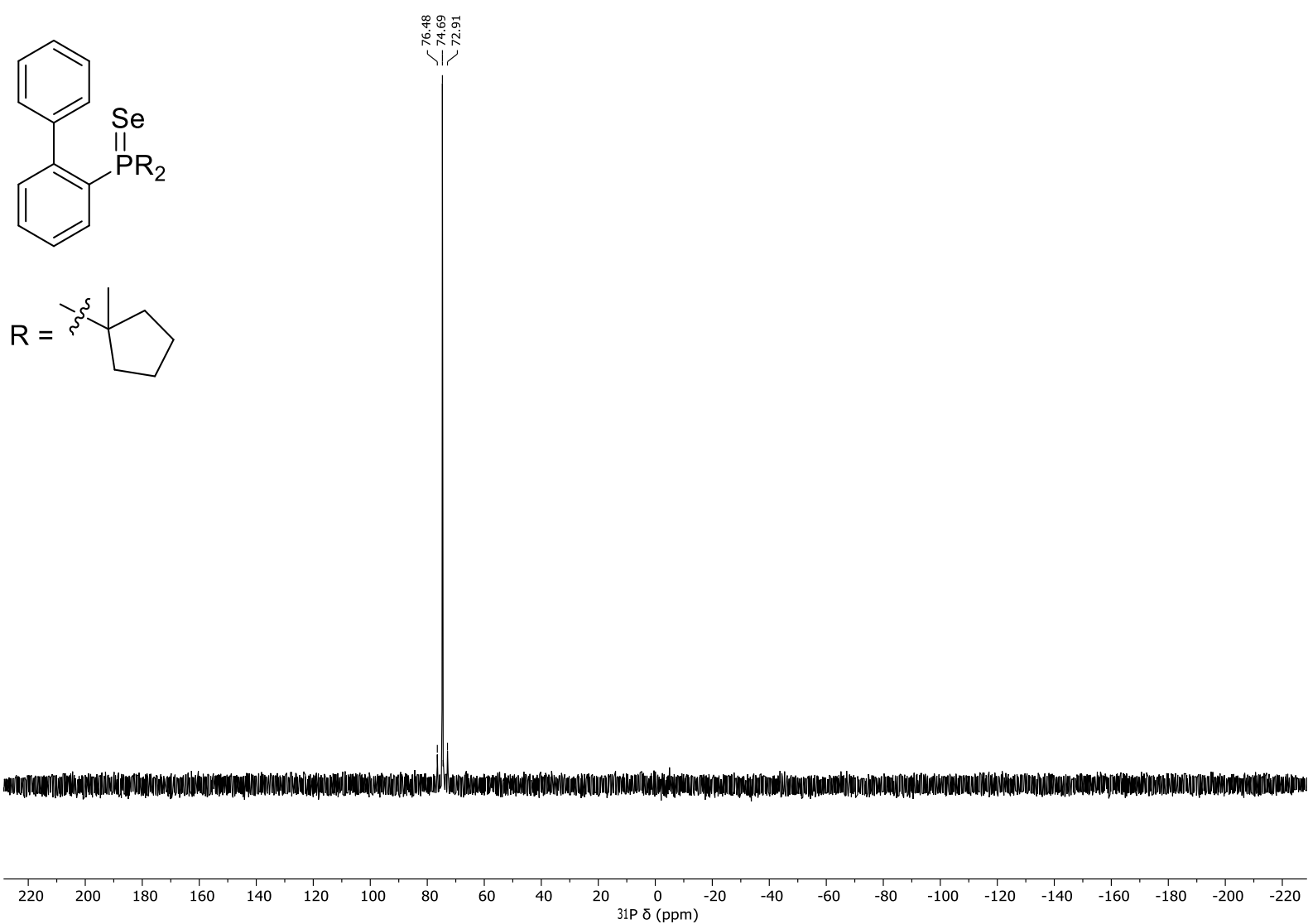
(2-Biphenyl)di-(1-methylcyclohexyl)phosphine selenide (4i) reaction mixture ${ }^{31} \mathrm{P}\left\{{ }^{1} \mathrm{H}\right\} \mathrm{NMR}(202 \mathrm{MHz}$, $\left.\mathrm{CDCl}_{3}\right)$

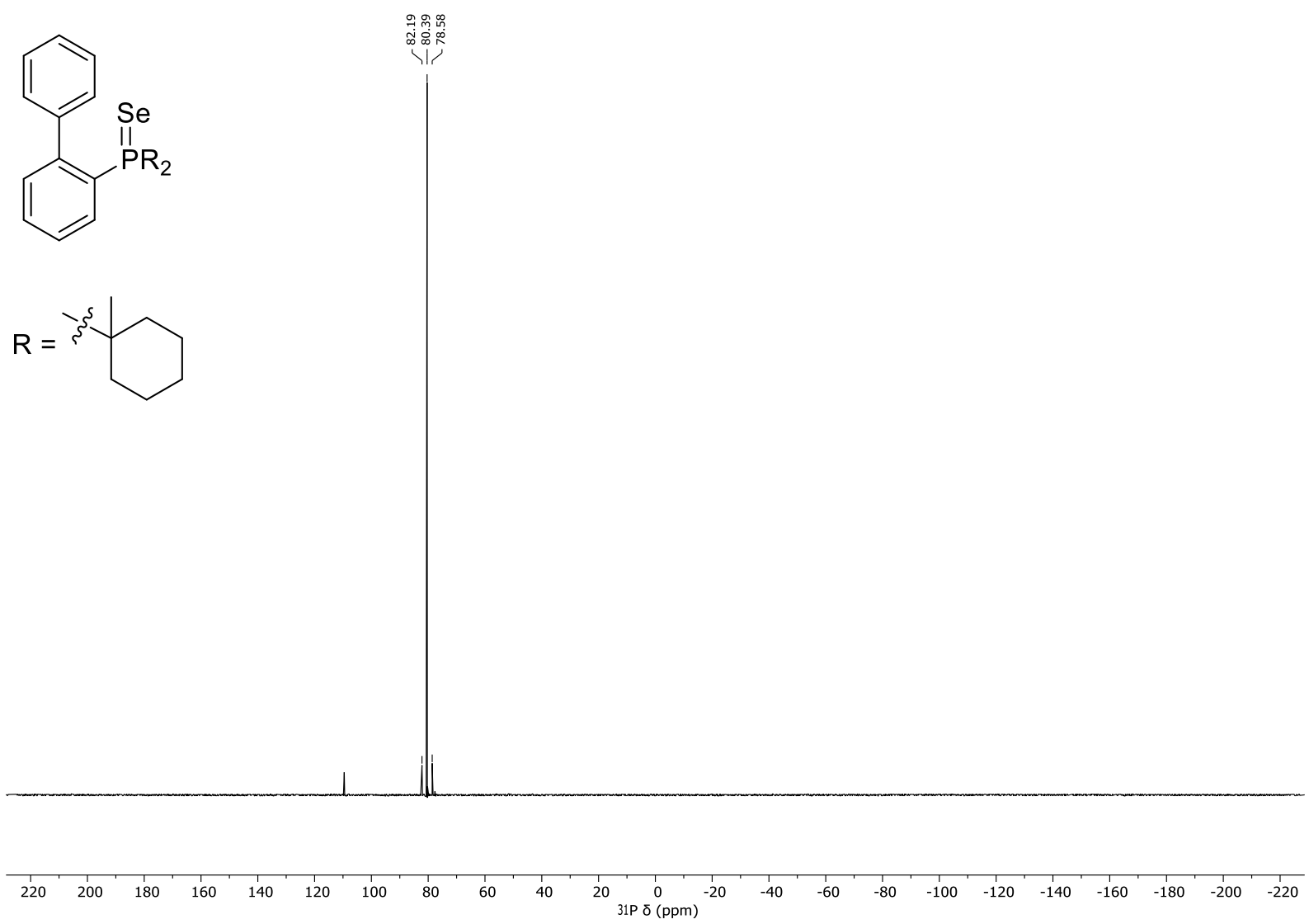


(2-Biphenyl)di-(1-adamantyl)phosphine selenide (4k) reaction mixture ${ }^{31} \mathrm{P}\left\{{ }^{1} \mathrm{H}\right\} \mathrm{NMR}(202 \mathrm{MHz}$, $\left.\mathrm{CDCl}_{3}\right)$

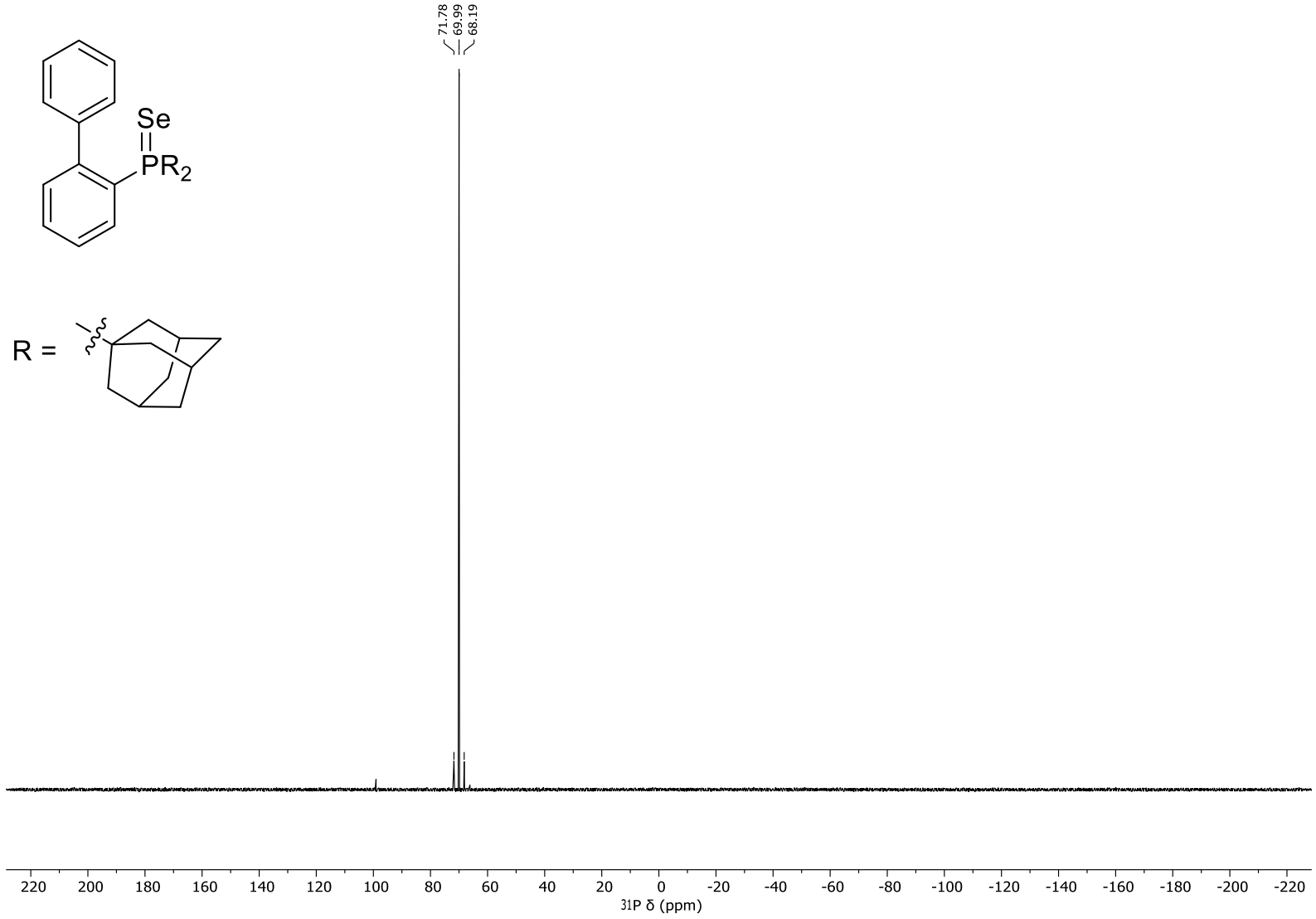


(2-Biphenyl)di-(2-methyl-2-adamantyl)phosphine selenide (4I) reaction mixture ${ }^{31} \mathrm{P}\left\{{ }^{1} \mathrm{H}\right\} \mathrm{NMR}$ (202 $\mathrm{MHz}, \mathrm{CDCl}_{3}$ )
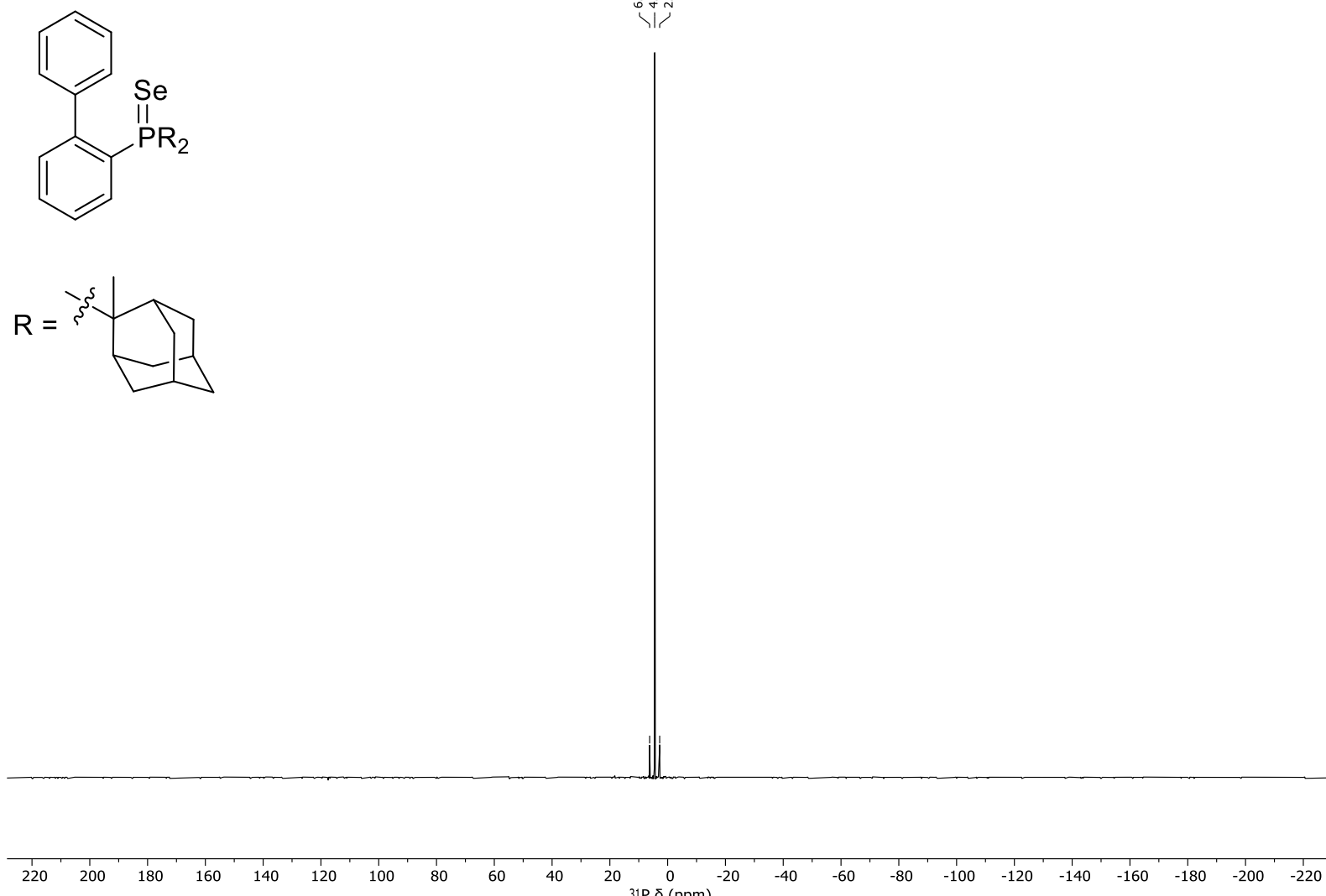
[(2-Biphenyl)di-tert-amylphosphine]gold(I) chloride (5a) - ${ }^{1} \mathrm{H}$ NMR $\left(500 \mathrm{MHz}, \mathrm{CDCl}_{3}\right)$

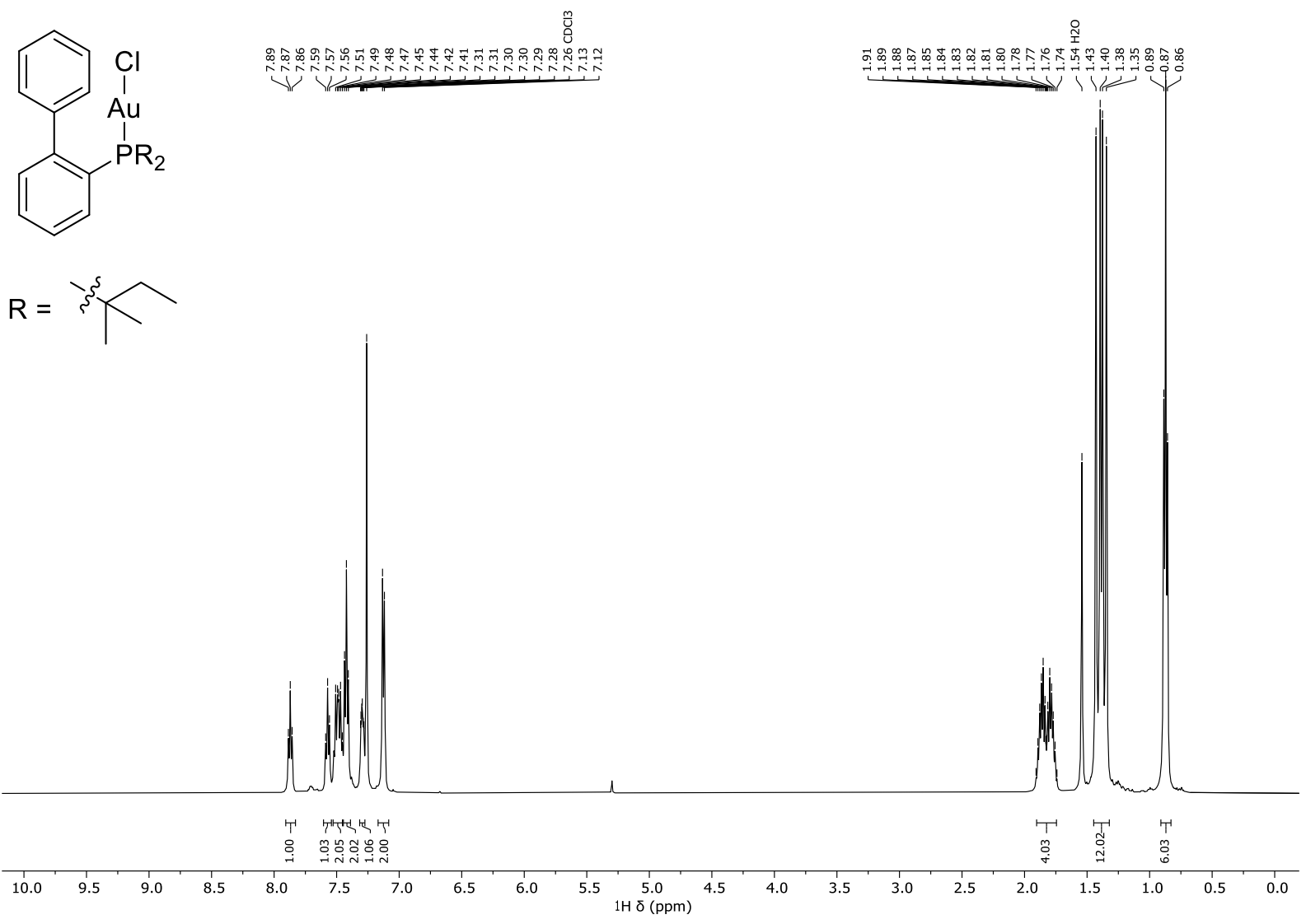

[(2-Biphenyl)di-tert-amylphosphine]gold(I) chloride (5a) - ${ }^{13} \mathrm{C}\left\{{ }^{1} \mathrm{H}\right\}$ NMR (126 MHz, $\left.\mathrm{CDCl}_{3}\right)$
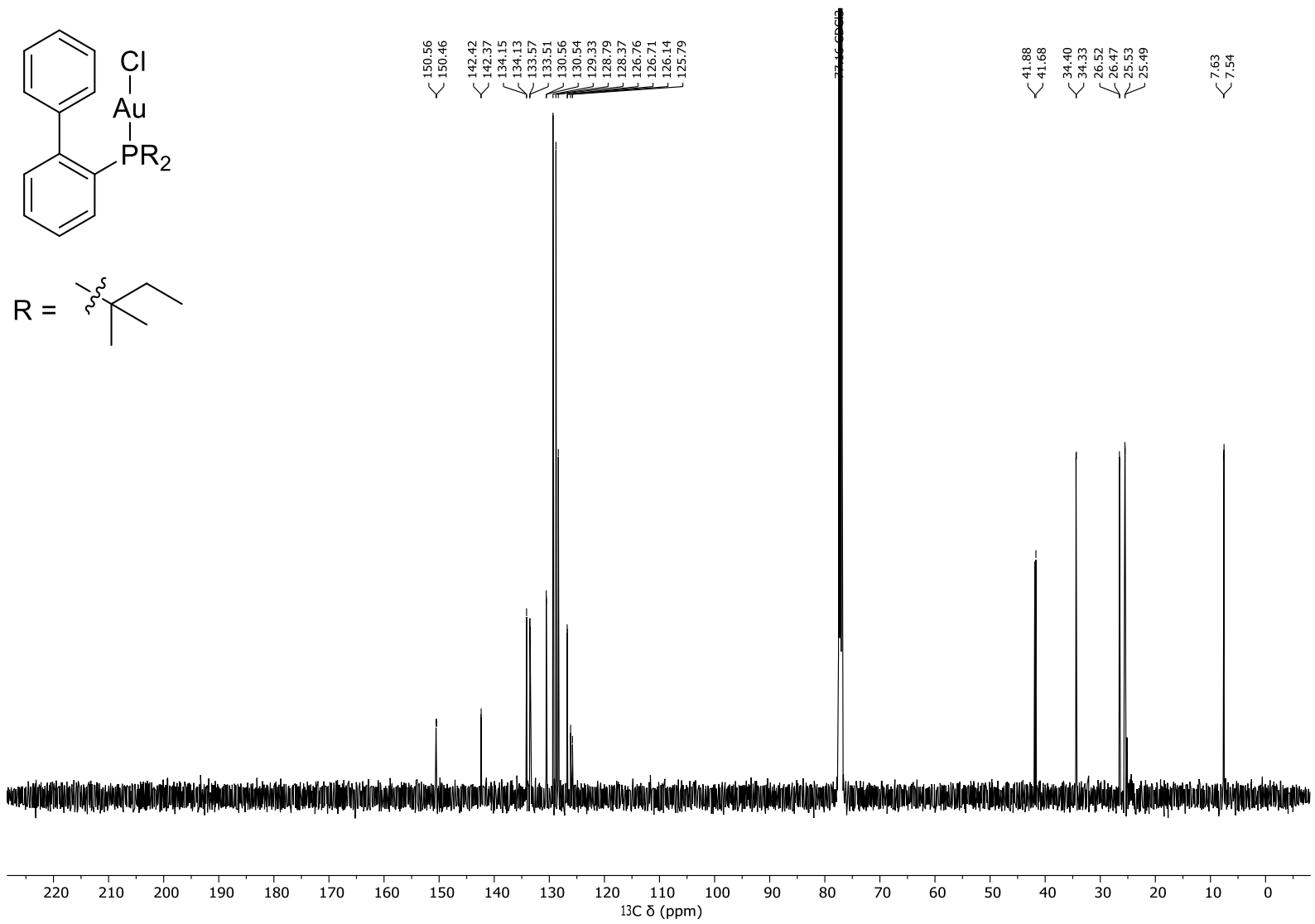
[(2-Biphenyl)di-tert-amylphosphine]gold(I) chloride (5a) ${ }^{31} \mathrm{P}\left\{{ }^{1} \mathrm{H}\right\} \mathrm{NMR}\left(202 \mathrm{MHz}, \mathrm{CDCl}_{3}\right)$

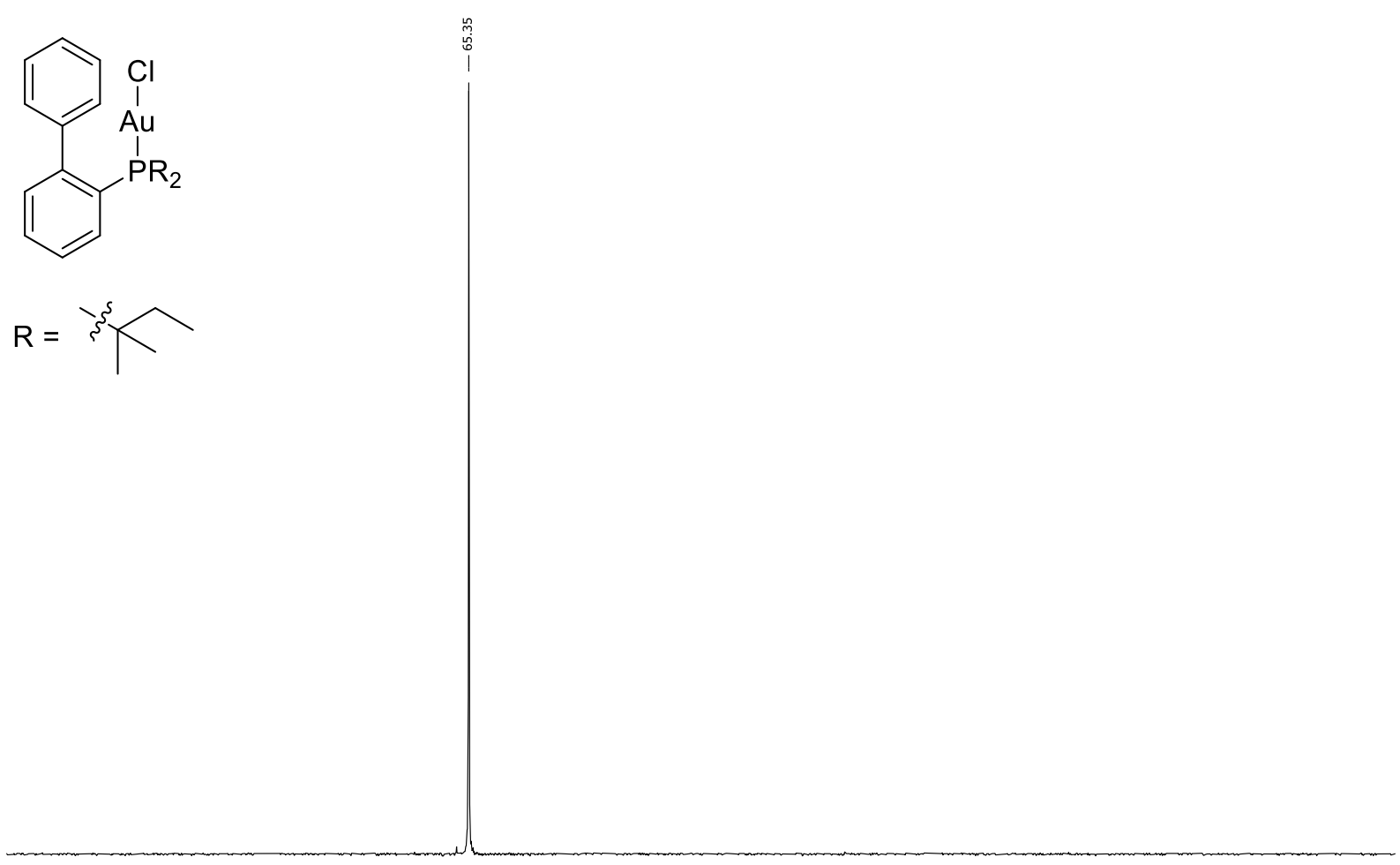

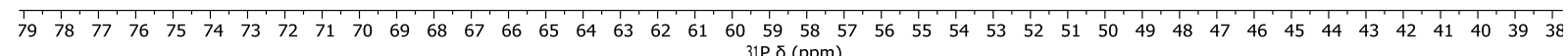


[(2-Biphenyl)di-(3-methyl-3-pentyl)phosphine]gold(I) chloride (5b) - ${ }^{1} \mathrm{H}$ NMR (500 MHz, $\left.\mathrm{CDCl}_{3}\right)$

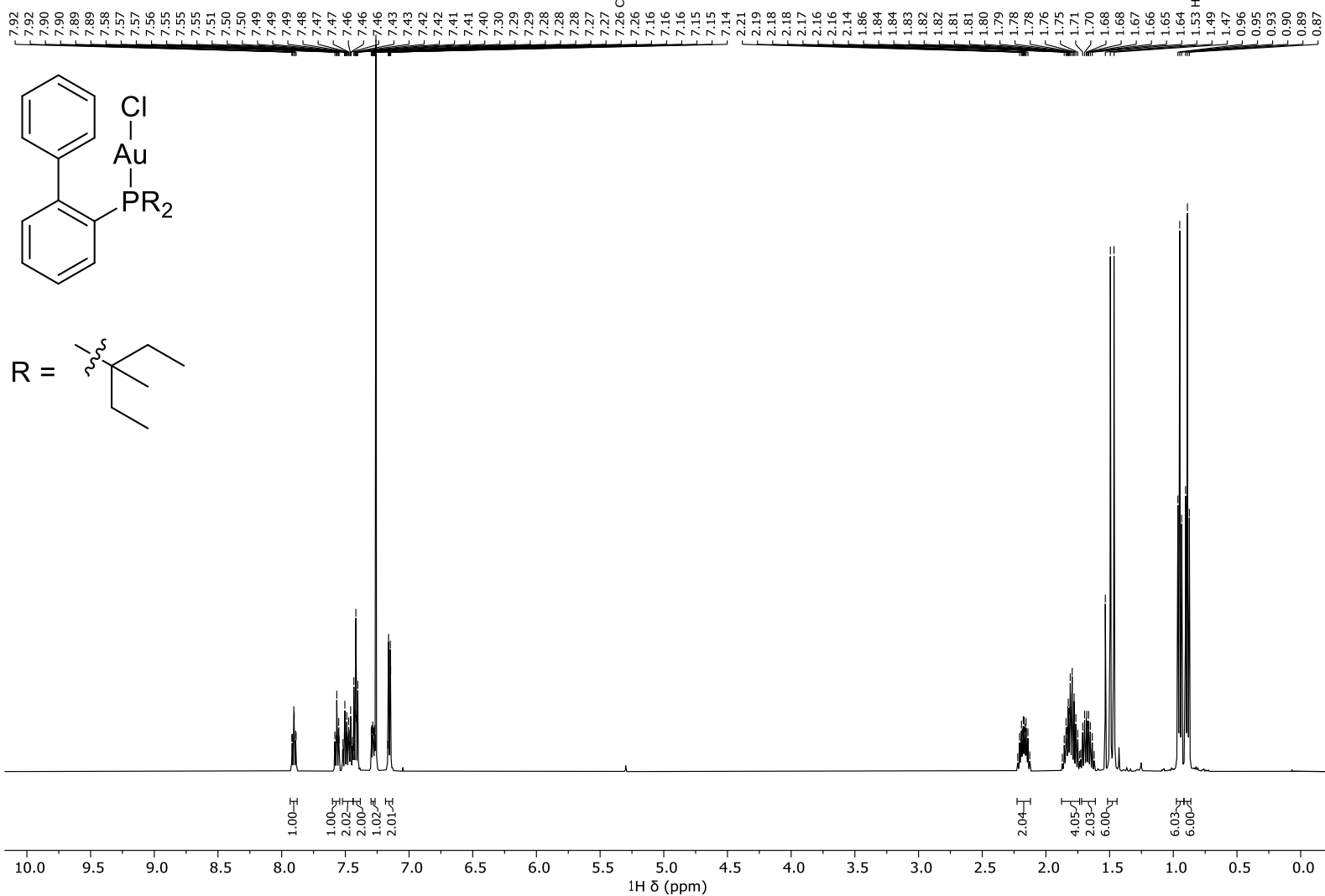

[(2-Biphenyl)di-(3-methyl-3-pentyl)phosphine]gold(I) chloride (5b) - ${ }^{13} \mathrm{C}\left\{{ }^{1} \mathrm{H}\right\} \mathrm{NMR}\left(126 \mathrm{MHz}, \mathrm{CDCl}_{3}\right)$
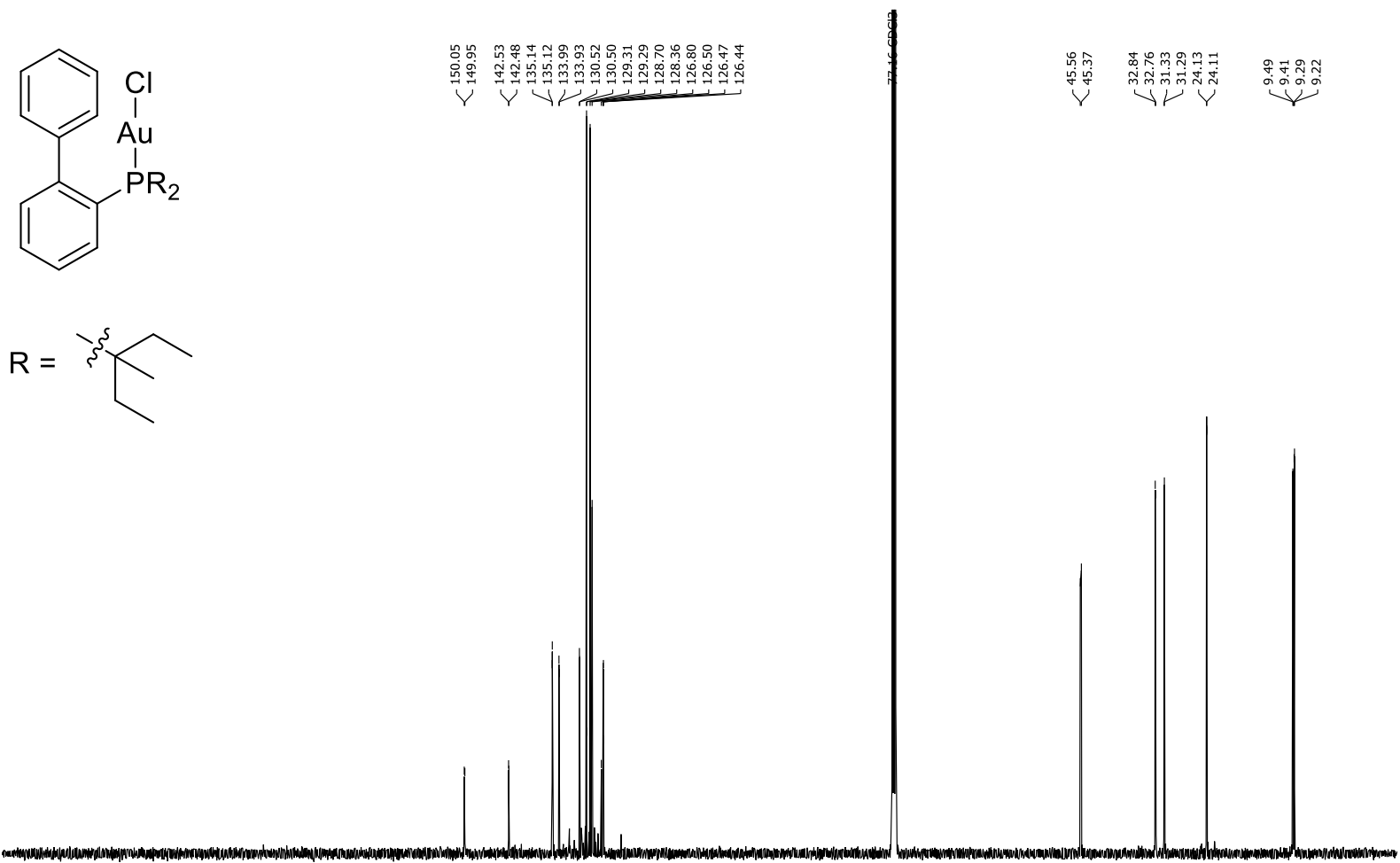

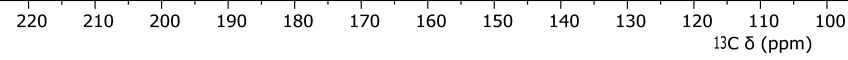


[(2-Biphenyl)di-(3-methyl-3-pentyl)phosphine]gold(I) chloride (5b) ${ }^{31} \mathrm{P}\left\{{ }^{1} \mathrm{H}\right\}$ NMR (202 $\left.\mathrm{MHz}, \mathrm{CDCl}_{3}\right)$

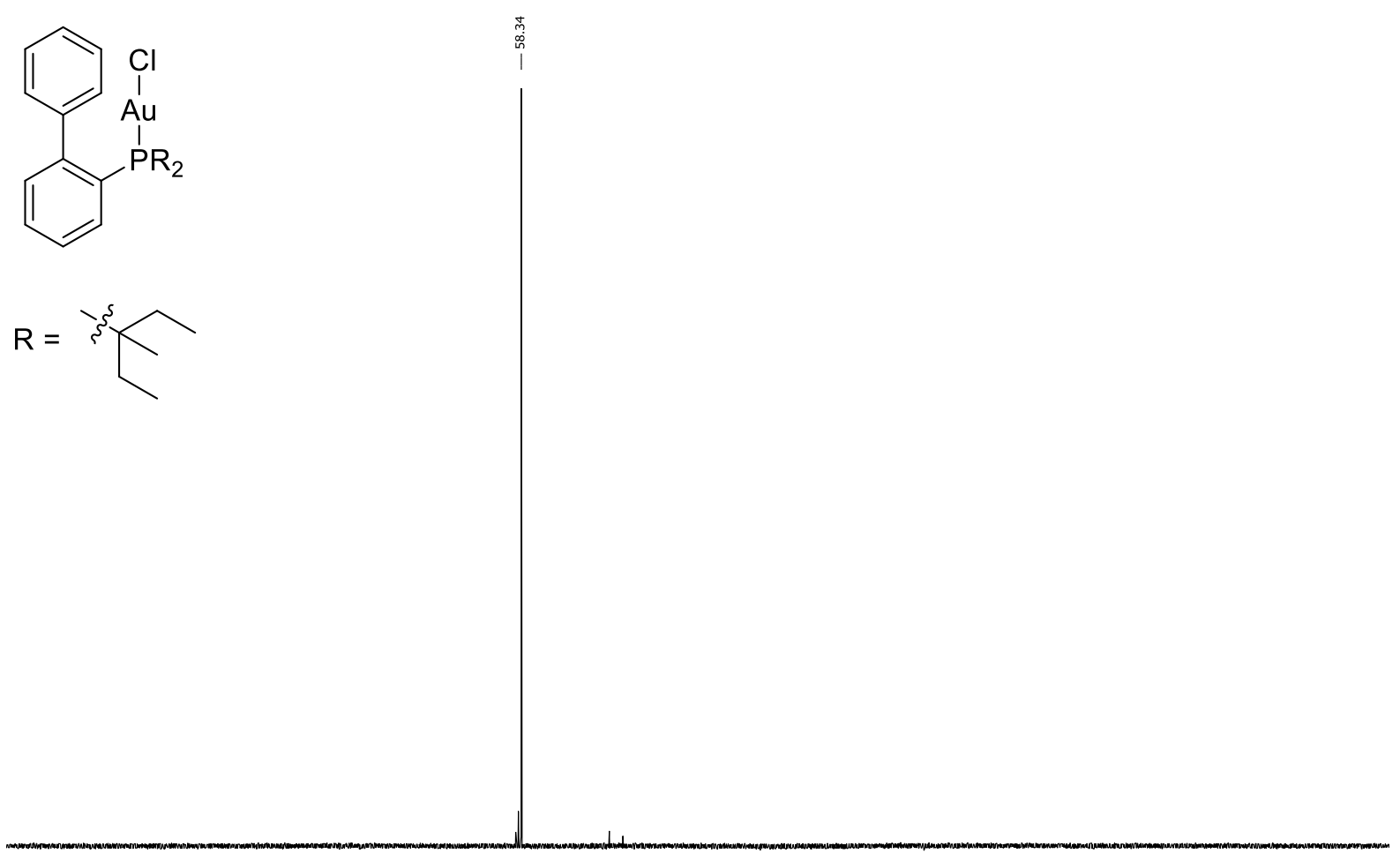

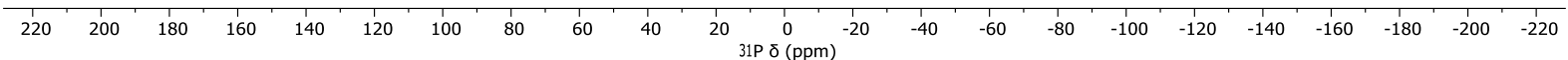


[(2-Biphenyl)di-(3-ethyl-3-pentyl)phosphine]gold(I) chloride (5c) - ${ }^{1} \mathrm{H}$ NMR (500 MHz, $\left.\mathrm{CDCl}_{3}\right)$

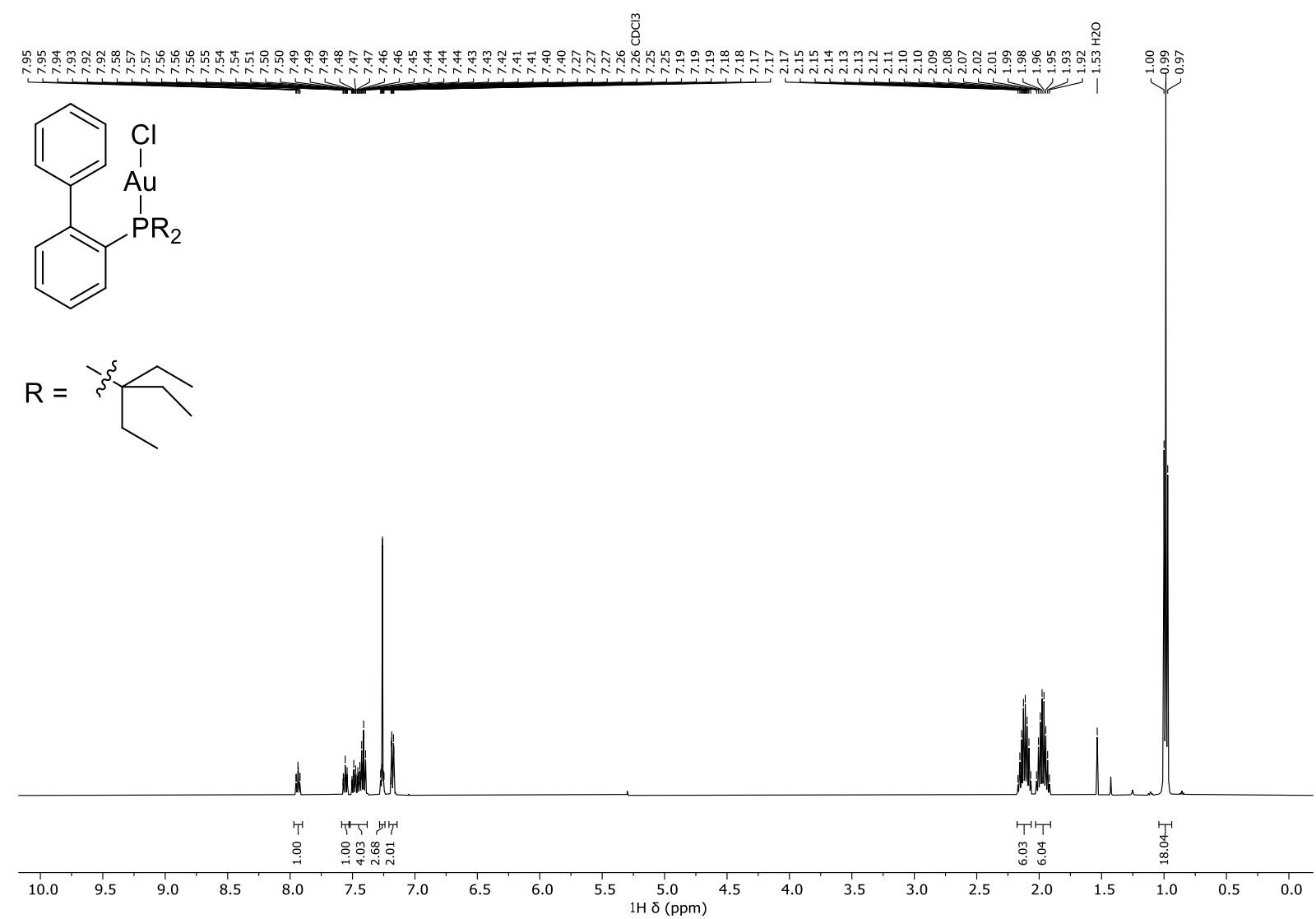

[(2-Biphenyl)di-(3-ethyl-3-pentyl)phosphine]gold(I) chloride (5c) - ${ }^{13} \mathrm{C}\left\{{ }^{1} \mathrm{H}\right\} \mathrm{NMR}\left(126 \mathrm{MHz}, \mathrm{CDCl}_{3}\right)$
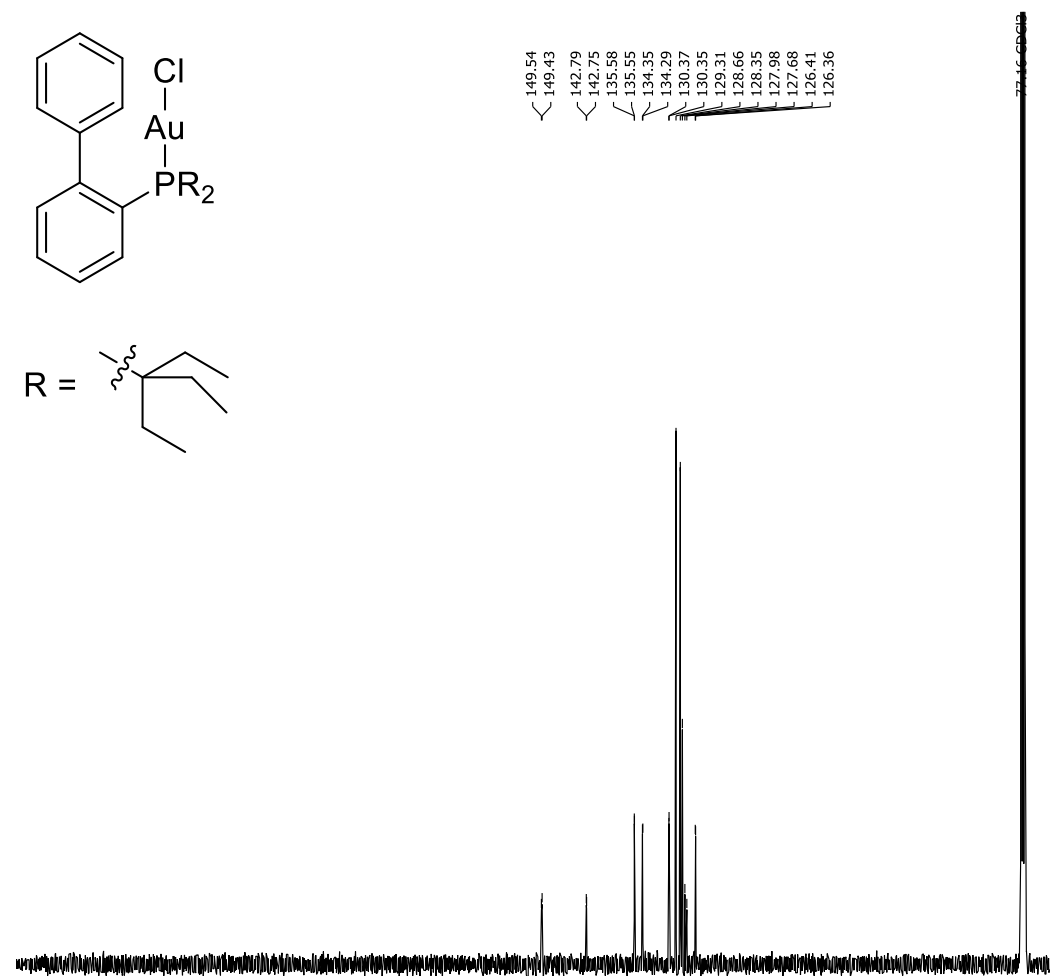
[(2-Biphenyl)di-(3-ethyl-3-pentyl)phosphine]gold(I) chloride (5c) ${ }^{31} \mathrm{P}\left\{{ }^{1} \mathrm{H}\right\}$ NMR (202 $\left.\mathrm{MHz}, \mathrm{CDCl}_{3}\right)$

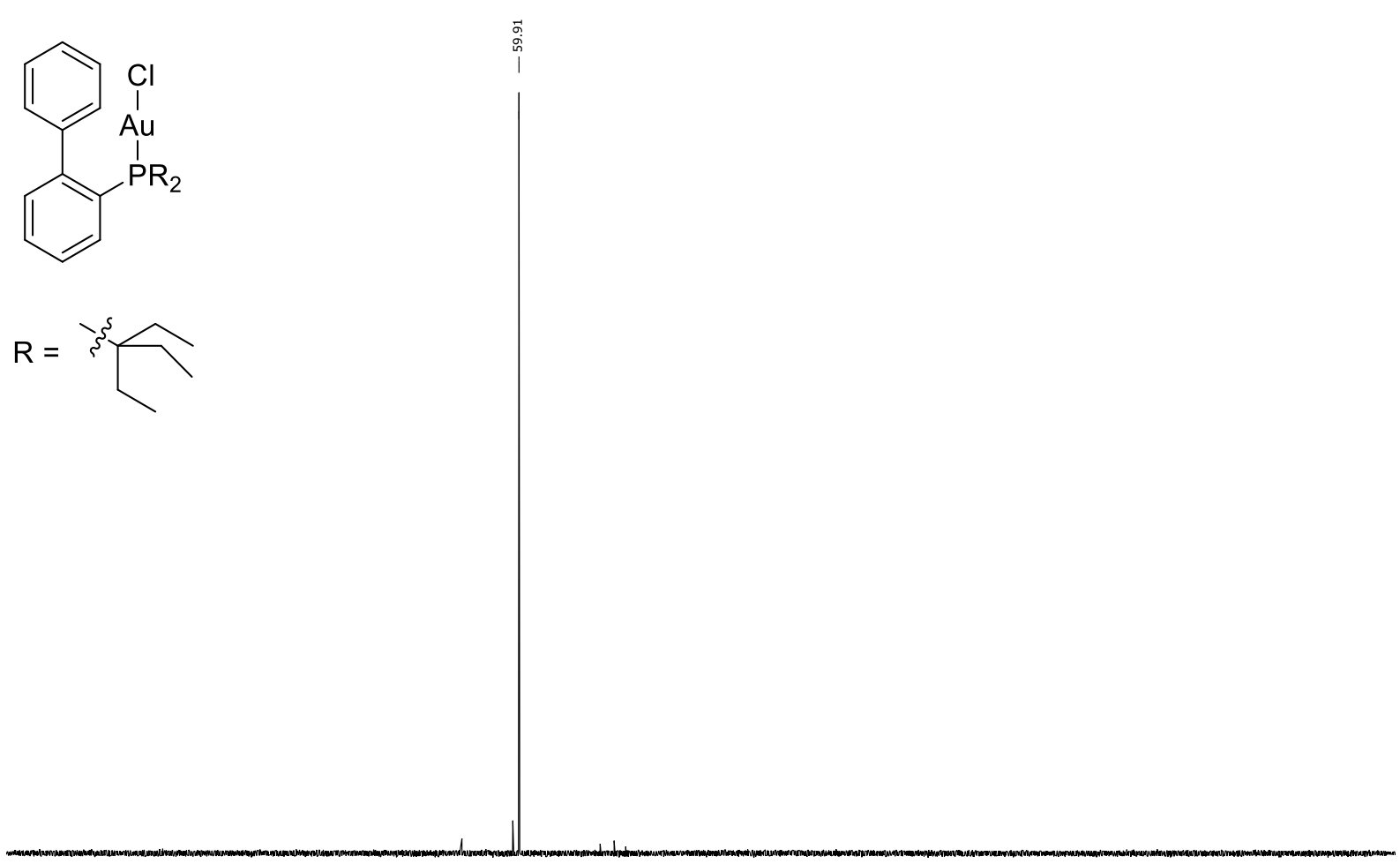

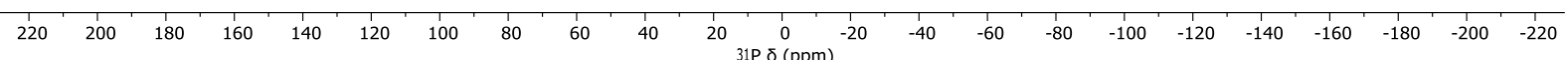


[(2-Biphenyl)bis-(2,3-dimethyl-2-butyl)phosphine]gold(I) chloride (5d) - ${ }^{1} \mathrm{H}$ NMR (500 MHz, $\left.\mathrm{CDCl}_{3}\right)$

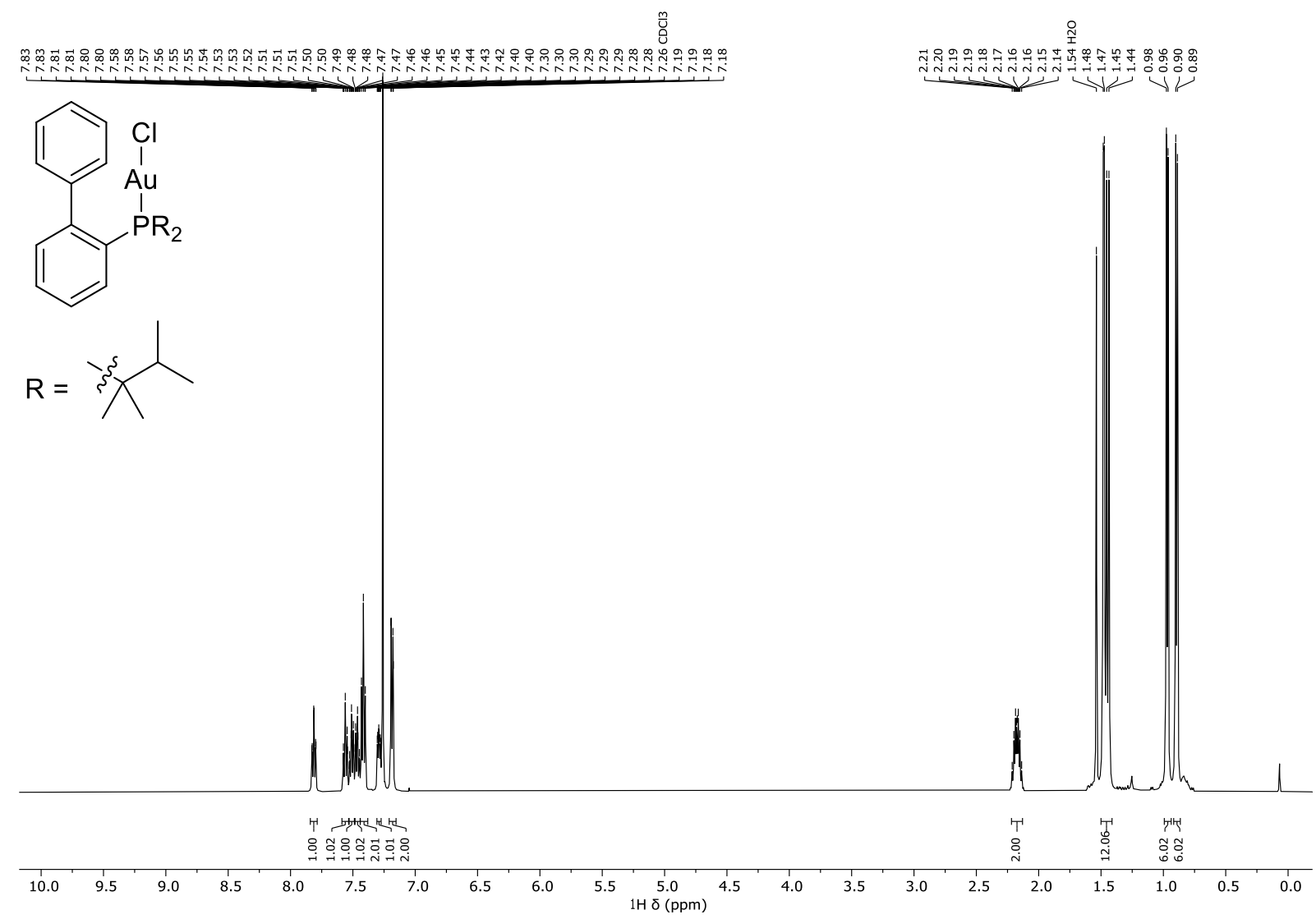

[(2-Biphenyl)bis-(2,3-dimethyl-2-butyl)phosphine]gold(I) chloride (5d) - ${ }^{13} \mathrm{C}\left\{{ }^{1} \mathrm{H}\right\} \mathrm{NMR}\left(126 \mathrm{MHz}, \mathrm{CDCl}_{3}\right)$

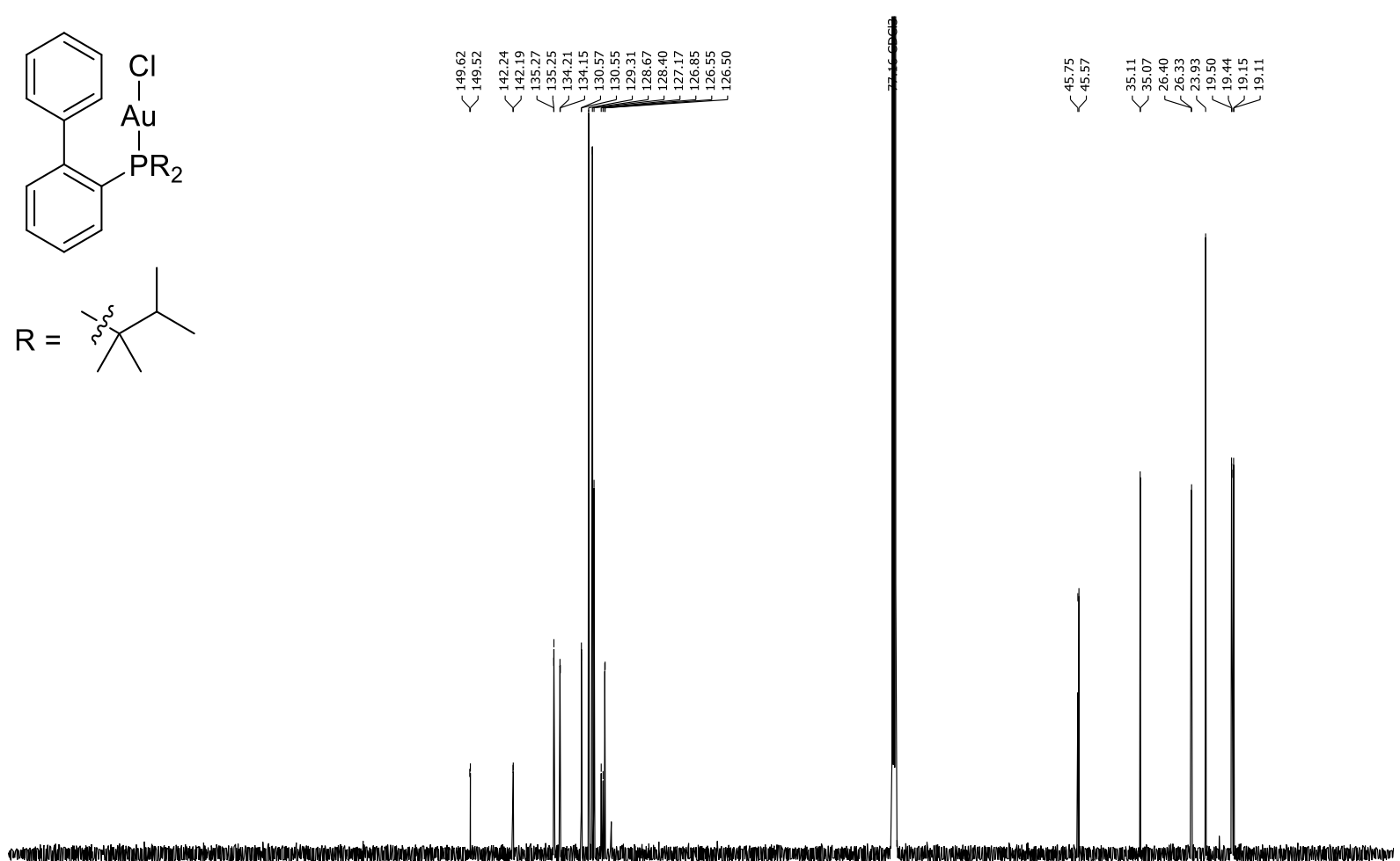

$\begin{array}{lllllllllllllllllllllll}220 & 210 & 200 & 190 & 180 & 170 & 160 & 150 & 140 & 130 & 120 & 110 & 100 & 90 & 80 & 70 & 60 & 50 & 40 & 30 & 20 & 10 & 0\end{array}$ 
[(2-Biphenyl)bis-(2,3-dimethyl-2-butyl)phosphine]gold(I) chloride (5d) ${ }^{31} \mathrm{P}\left\{{ }^{1} \mathrm{H}\right\} \mathrm{NMR}$ (202 MHz, $\left.\mathrm{CDCl}_{3}\right)$

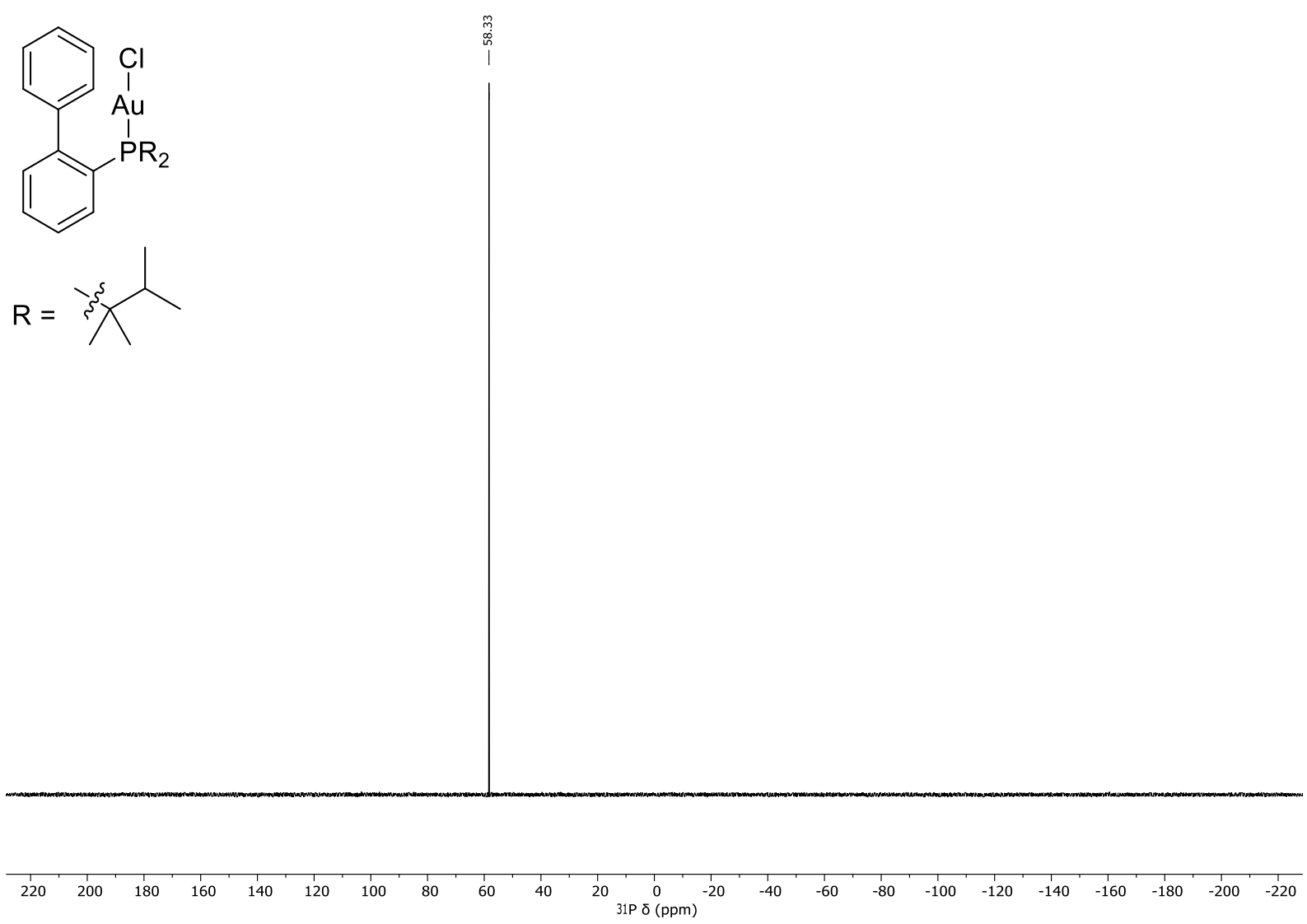


[(2-Biphenyl)di-(2-methyl-4-phenyl-2-butyl)phosphine]gold(I) chloride (5f) - ${ }^{1} \mathrm{H} N M R\left(500 \mathrm{MHz}, \mathrm{CDCl}_{3}\right)$

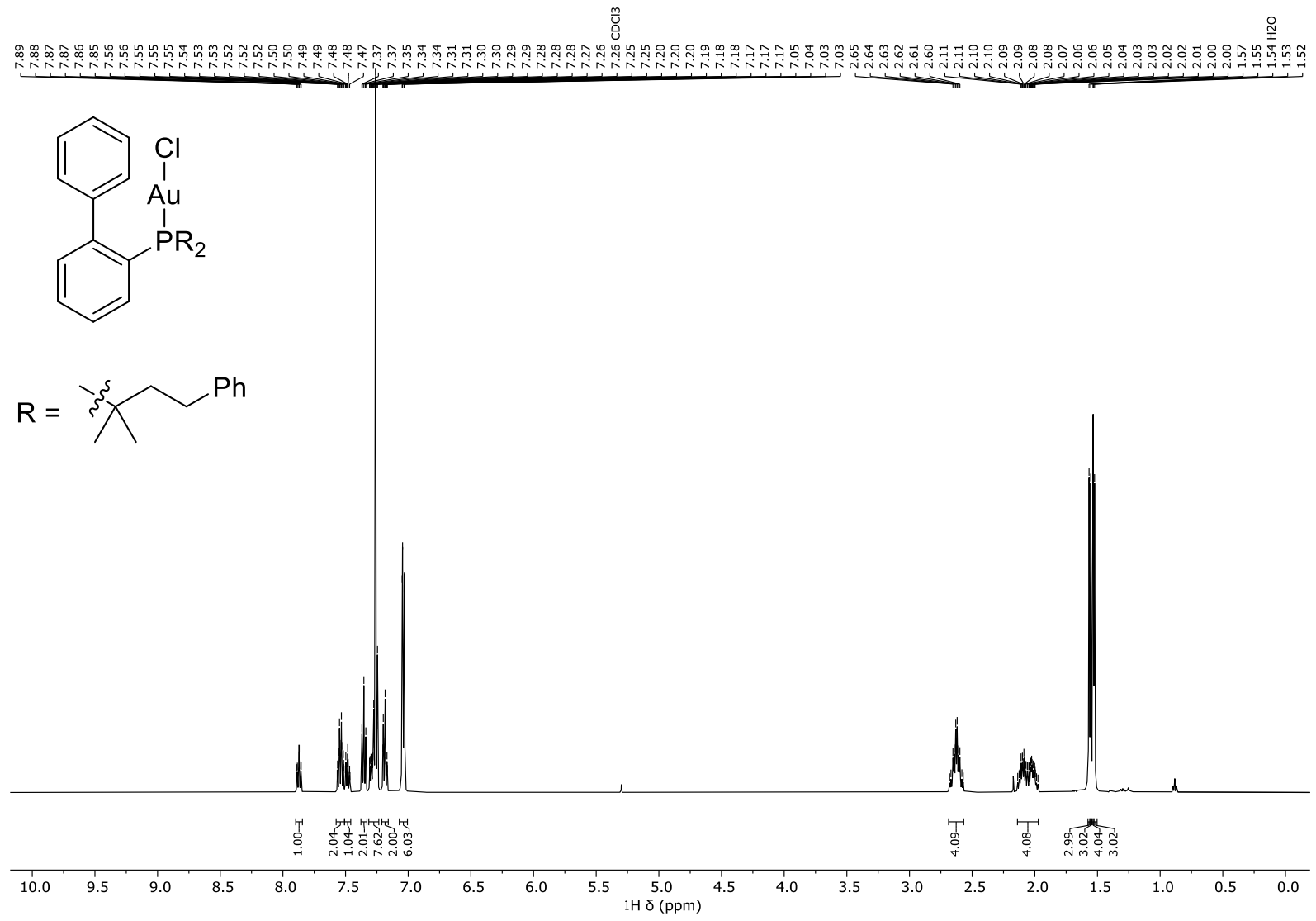

[(2-Biphenyl)di-(2-methyl-4-phenyl-2-butyl)phosphine]gold(I) chloride (5f) - ${ }^{13} \mathrm{C}\left\{{ }^{1} \mathrm{H}\right\} \mathrm{NMR}\left(126 \mathrm{MHz}, \mathrm{CDCl}_{3}\right)$<smiles>[R2]c1ccccc1-c1ccccc1</smiles>
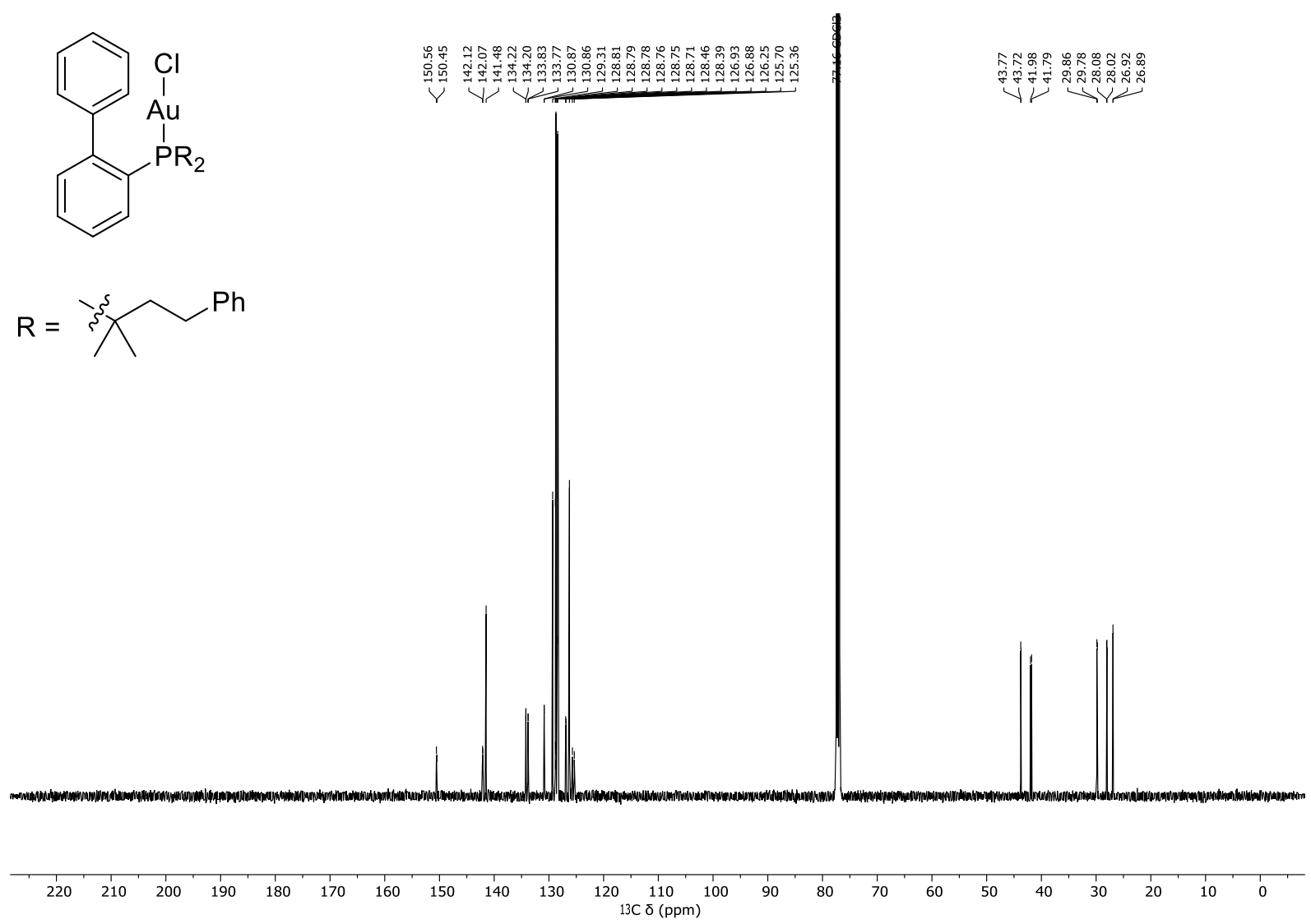

S200 
[(2-Biphenyl)di-(2-methyl-4-phenyl-2-butyl)phosphine]gold(I) chloride (5f) ${ }^{31} \mathrm{P}\left\{{ }^{1} \mathrm{H}\right\} \mathrm{NMR}(202 \mathrm{MHz}$, $\mathrm{CDCl}_{3}$

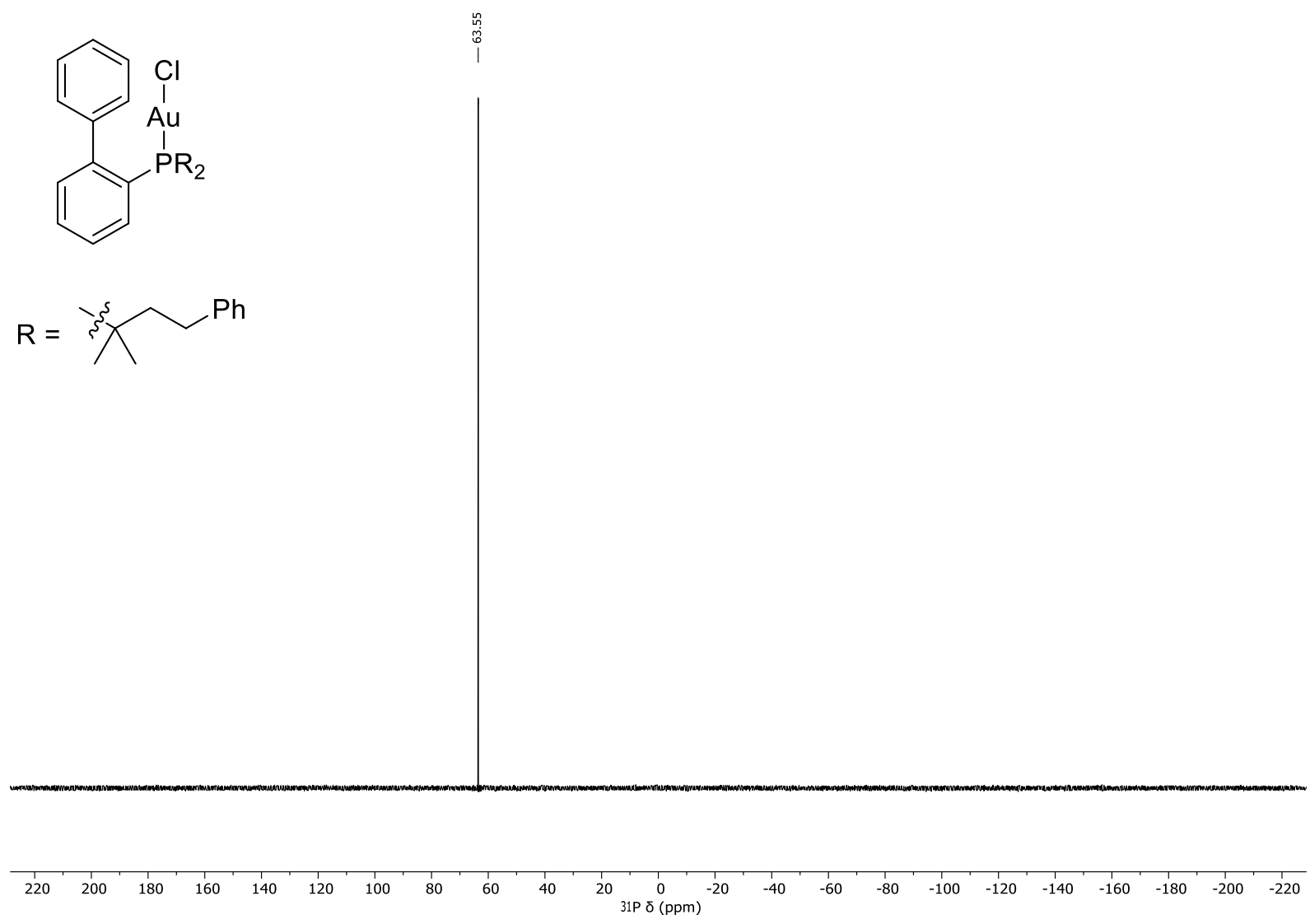


[(2-Biphenyl)di-(1-methylcyclopentyl)phosphine]gold(I) chloride (5h) - ${ }^{1} \mathrm{H} \mathrm{NMR}\left(500 \mathrm{MHz}, \mathrm{CDCl}_{3}\right)$

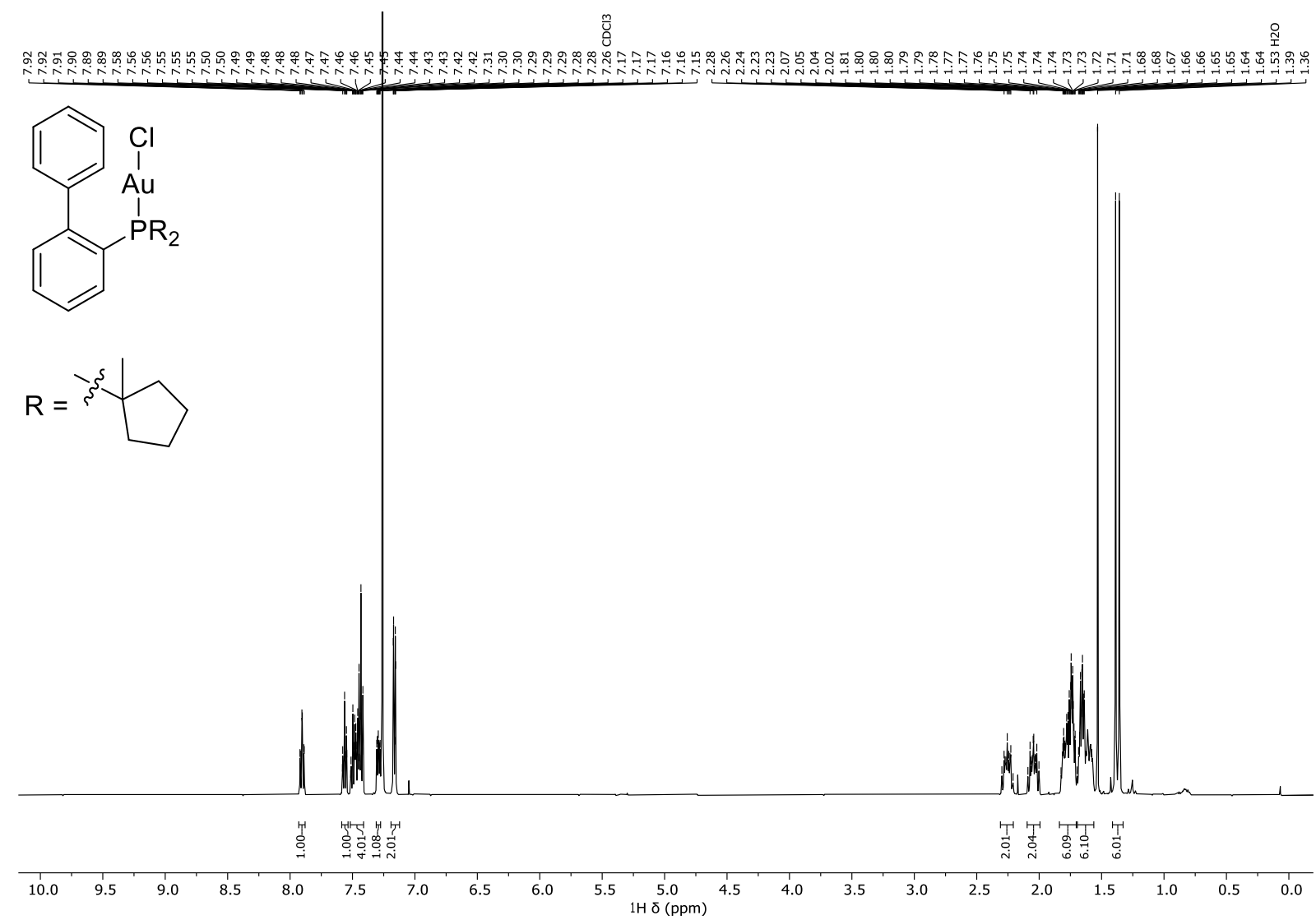

[(2-Biphenyl)di-(1-methylcyclopentyl)phosphine]gold(I) chloride (5h) - ${ }^{13} \mathrm{C}\left\{{ }^{1} \mathrm{H}\right\} \mathrm{NMR}\left(126 \mathrm{MHz}, \mathrm{CDCl}_{3}\right)$

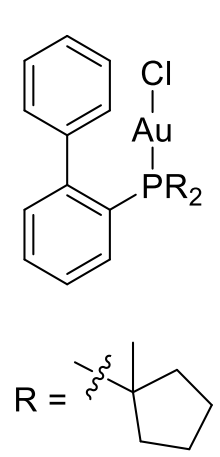

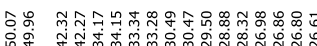

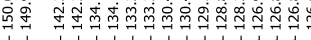

$\checkmark$ Y

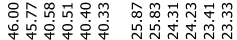

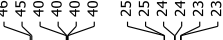

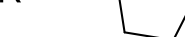

$\begin{array}{llllllllllll}220 & 210 & 200 & 190 & 180 & 170 & 160 & 150 & 140 & 130 & 120 \underset{13 \mathrm{C} \delta(\mathrm{ppm})}{110}\end{array}$ 
[(2-Biphenyl)di-(1-methylcyclopentyl)phosphine]gold(I) chloride $(5 \mathrm{~h}){ }^{31} \mathrm{P}\left\{{ }^{1} \mathrm{H}\right\} \mathrm{NMR}\left(202 \mathrm{MHz}, \mathrm{CDCl}_{3}\right)$

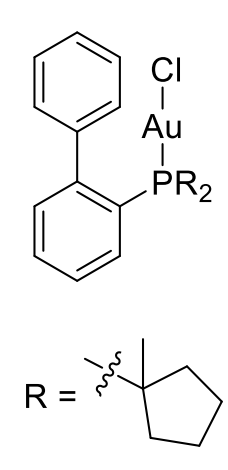

$\begin{array}{lllllllllll}1 & 1 & 1 & 1 & 1 & 1 & 1 & 1 & 1 & 1 & 1 \\ 200 & 200 & 180 & 160 & 140 & 120 & 100 & 80 & 60 & 40 & 20 \\ 31 \mathrm{P} \delta(\mathrm{ppm}) & -20\end{array}$ 
[(2-Biphenyl)di-(1-methylcyclohexyl)phosphine]gold(I) chloride (5i) - ${ }^{1} \mathrm{H}$ NMR $\left(500 \mathrm{MHz}, \mathrm{CDCl}_{3}\right)$

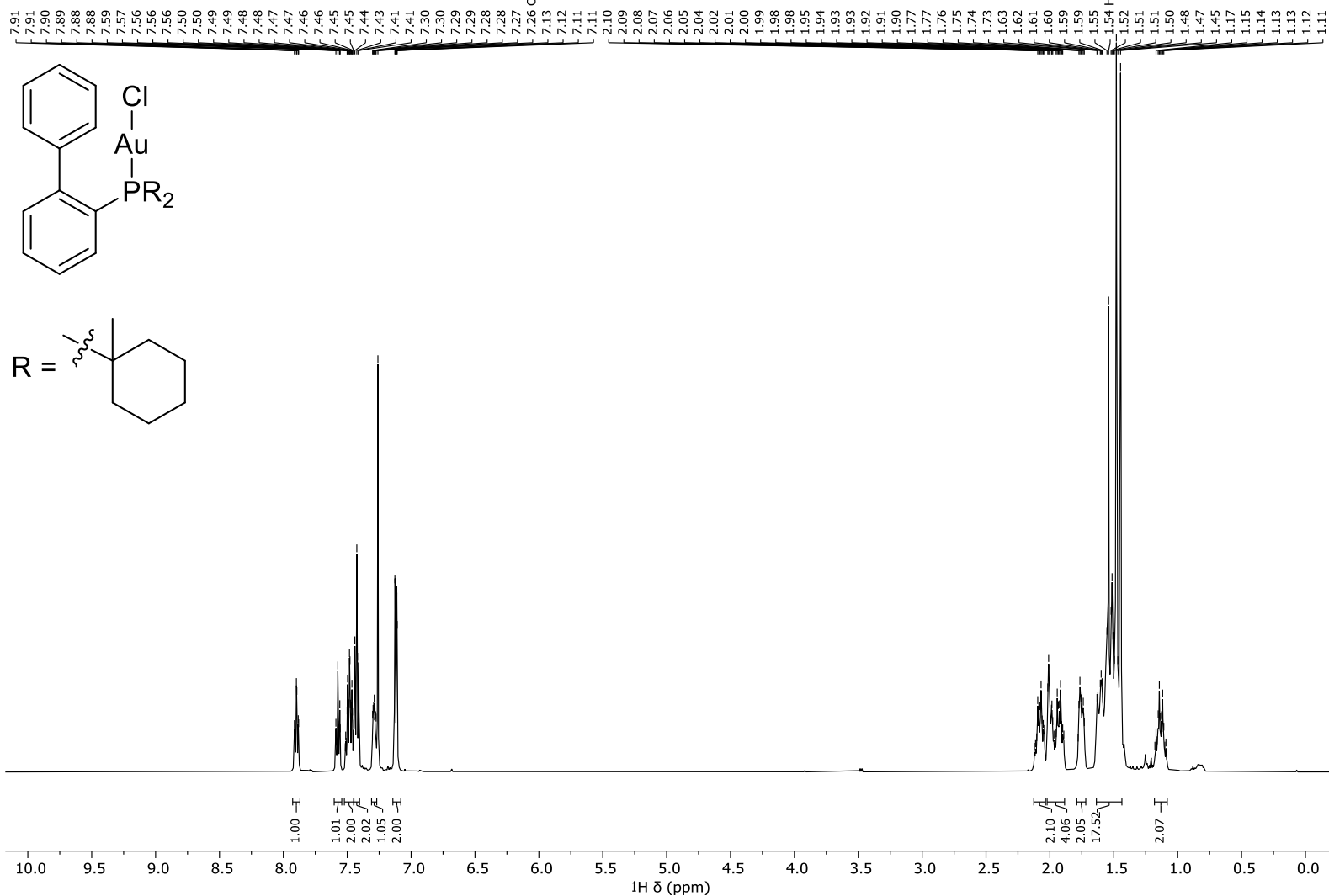

[(2-Biphenyl)di-(1-methylcyclohexyl)phosphine]gold(I) chloride (5i) - ${ }^{13} \mathrm{C}\left\{{ }^{1} \mathrm{H}\right\} \mathrm{NMR}\left(126 \mathrm{MHz}, \mathrm{CDCl}_{3}\right)$

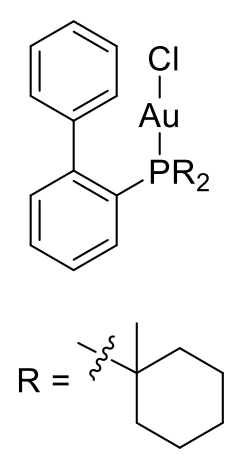

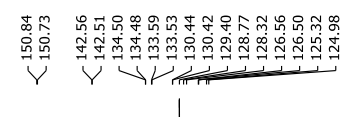

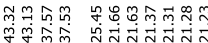

$\checkmark Y$ N

L

$\begin{array}{lllllllllll}220 & 210 & 200 & 190 & 180 & 170 & 160 & 150 & 140 & 130 & 120 \\ 13 \mathrm{C} \delta(\mathrm{ppm}) & 100\end{array}$ 
[(2-Biphenyl)di-(1-methylcyclohexyl)phosphine]gold(I) chloride (5i) ${ }^{31} \mathrm{P}\left\{{ }^{1} \mathrm{H}\right\}$ NMR $\left(202 \mathrm{MHz}, \mathrm{CDCl}_{3}\right)$

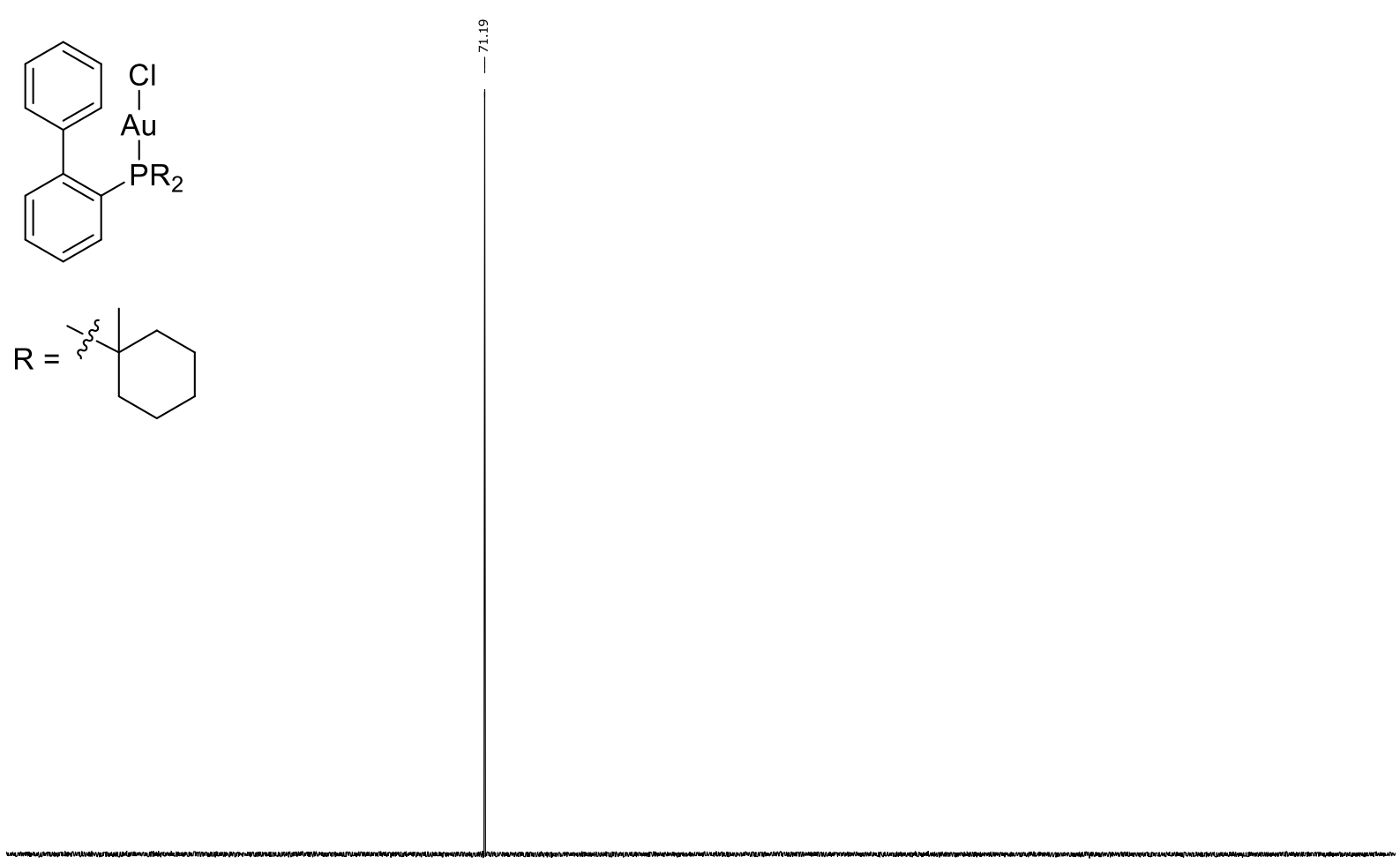

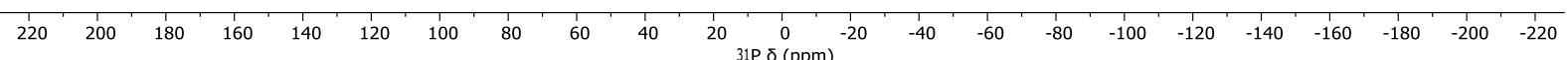


[(2-Biphenyl)di-(1-adamantyl)phosphine]gold(I) chloride (5k) - ${ }^{1} \mathrm{H} \mathrm{NMR}\left(500 \mathrm{MHz}, \mathrm{CDCl}_{3}\right)$

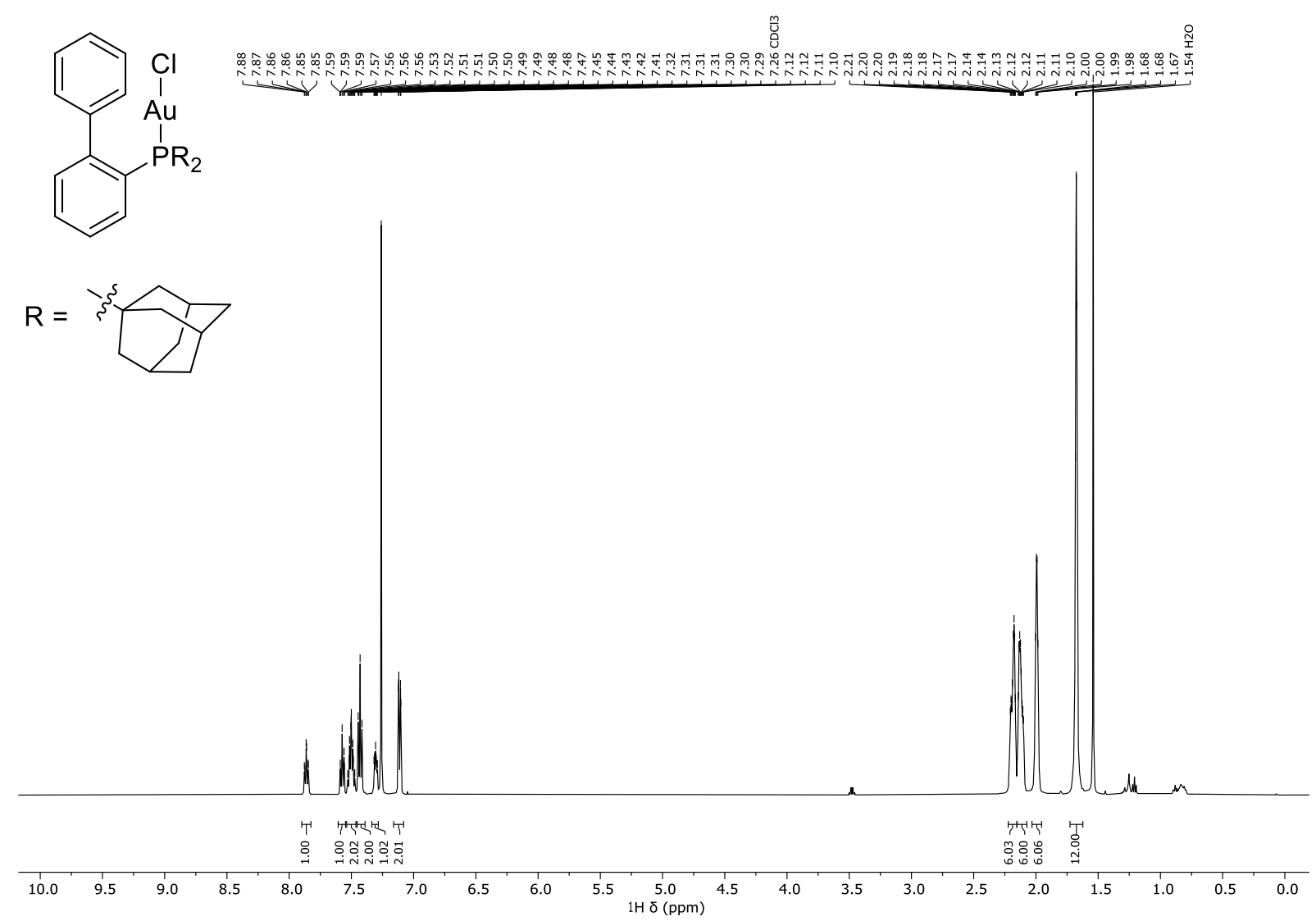

[(2-Biphenyl)di-(1-adamantyl)phosphine]gold(I) chloride (5k) - ${ }^{13} \mathrm{C}\left\{{ }^{1} \mathrm{H}\right\}$ NMR (126 MHz, $\left.\mathrm{CDCl}_{3}\right)$
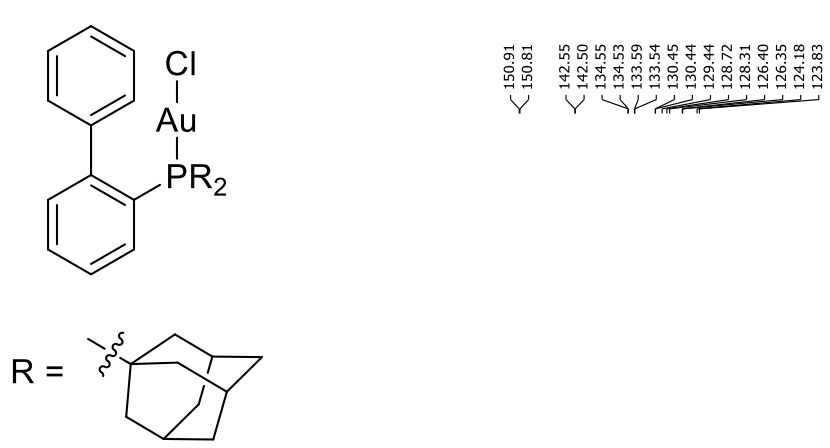

Y

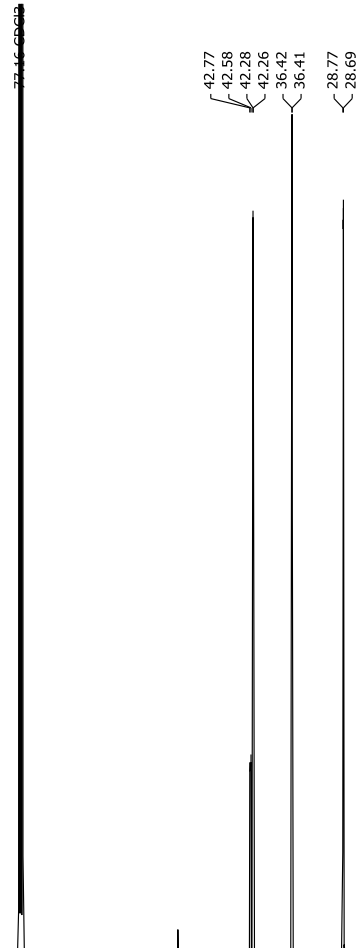

$\begin{array}{lllllllllll}220 & 210 & 200 & 190 & 180 & 170 & 160 & 150 & 140 & 130 & 120 \\ 13 \mathrm{C} \delta(\mathrm{ppm}) & 100\end{array}$ 
[(2-Biphenyl)di-(1-adamantyl)phosphine]gold(I) chloride (5k) ${ }^{31} \mathrm{P}\left\{{ }^{1} \mathrm{H}\right\} \mathrm{NMR}\left(202 \mathrm{MHz}, \mathrm{CDCl}_{3}\right)$

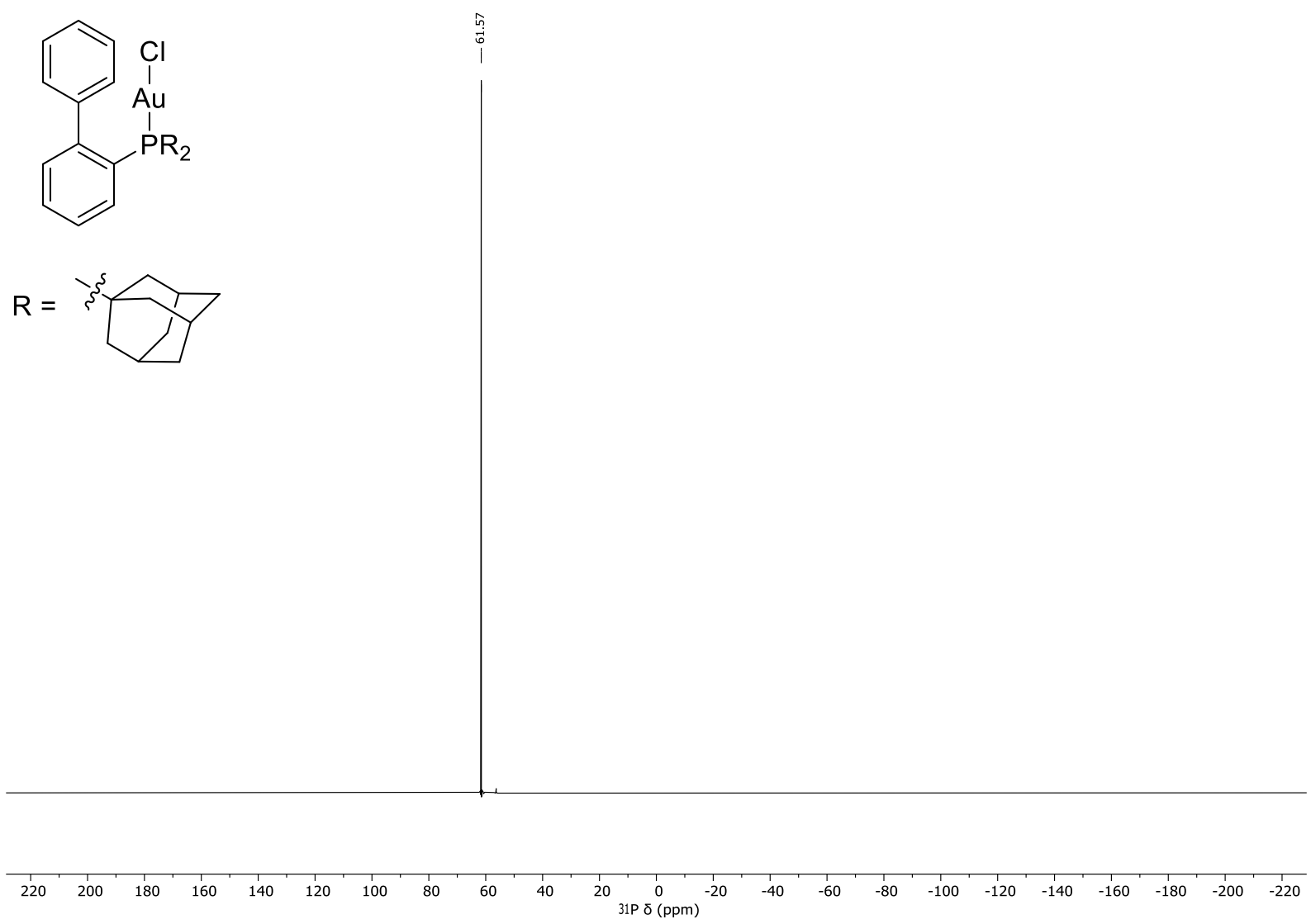




\section{References}

(1) Jin, M.; Adak, L.; Nakamura, M. Iron-Catalyzed Enantioselective Cross-Coupling Reactions of $\alpha$-Chloroesters with Aryl Grignard Reagents. J. Am. Chem. Soc. 2015, 137, 7128-7134.

(2) Saito, T.; Nishimoto, Y.; Yasuda, M.; Baba, A. InCl ${ }_{3}$-Catalyzed Cross-Coupling of Alkyl Trimethylsilyl Ethers and Allylsilanes via an in Situ Derived Combined Lewis Acid of $\mathrm{InCl}_{3}$ and $\mathrm{Me}_{3} \mathrm{Sil}$. J. Org. Chem. 2007, 72, 8588-8590.

(3) Vuluga, D.; Legros, J.; Crousse, B.; Bonnet-Delpon, D. Fluorous 4-N,N -Dimethylaminopyridine (DMAP) Salts as Simple Recyclable Acylation Catalysts. Chem. Eur. J. 2010, 16, 1776-1779.

(4) Ishihara, K.; Kubota, M.; Kurihara, H.; Yamamoto, H. Scandium Trifluoromethanesulfonate as an Extremely Active Lewis Acid Catalyst in Acylation of Alcohols with Acid Anhydrides and Mixed Anhydrides . J. Org. Chem. 1996, 61, 4560-4567.

(5) Strazzolini, P.; Giumanini, A. G.; Verardo, G. The Reaction between Acyl Halides and Alcohols: Alkyl Halide vs. Ester Formation. Tetrahedron 1994, 50, 217-254.

(6) Fujita, S.; Hayashi, Y.; Nômi, T.; Nozaki, H. Photochemical Addition of Protic Solvents to 1Phenylcycloalkenes. Tetrahedron 1971, 27, 1607-1613.

(7) Liu, Z.; Ma, Q.; Liu, Y.; Wang, Q. 4-(N,N -Dimethylamino)Pyridine Hydrochloride as a Recyclable Catalyst for Acylation of Inert Alcohols: Substrate Scope and Reaction Mechanism. Org. Lett. 2014, 16, 236-239.

(8) Baldwin, S. W.; Haut, S. A. Reductive Deoxygenation of Esters with Trichlorosilane. J. Org. Chem. 1975, 40, 3885-3887.

(9) Xin, Z.; Gøgsig, T. M.; Lindhardt, A. T.; Skrydstrup, T. An Efficient Method for the Preparation of Tertiary Esters by Palladium-Catalyzed Alkoxycarbonylation of Aryl Bromides. Org. Lett. 2012, 14, 284-287.

(10) Mukaiyama, T.; Shintou, T.; Fukumoto, K. A Convenient Method for the Preparation of Inverted Tert -Alkyl Carboxylates from Chiral Tert -Alcohols by a New Type of Oxidation-Reduction Condensation Using 2,6-Dimethyl-1,4-Benzoquinone. J. Am. Chem. Soc. 2003, 125, 10538-10539.

(11) Brown, L.; Koreeda, M. Benzoyl Trifluoromethanesulfonate. A Mild Reagent for the Benzoylation of Sterically Hindered Hydroxyls. J. Org. Chem. 1984, 49, 3875-3880. 
(12) Gopalan, B.; Ponpandian, T.; Kachhadia, V.; Bharathimohan, K.; Vignesh, R.; Sivasudar, V.; Narayanan, S.; Mandar, B.; Praveen, R.; Saranya, N.; Rajagopal, S.; Rajagopal, S. Discovery of Adamantane Based Highly Potent HDAC Inhibitors. Bioorganic Med. Chem. Lett. 2013, 23, 2532-2537.

(13) Alder, R. W.; Carta, F.; Reed, C. A.; Stoyanova, I.; Willis, C. L. Searching for Intermediates in Prins Cyclisations: The 2-Oxa-5-Adamantyl Carbocation. Org. Biomol. Chem. 2010, 8, 1551-1559.

(14) Kolocouris, A.; Koch, A.; Kleinpeter, E.; Stylianakis, I. 2-Substituted and 2,2-Disubstituted Adamantane Derivatives as Models for Studying Substituent Chemical Shifts and $\mathrm{C}-\mathrm{H}_{\mathrm{ax}} \cdots \mathrm{Y}_{\mathrm{ax}}$ Cyclohexane Contacts - Results from Experimental and Theoretical NMR Spectroscopic Chemical Shifts and DFT Structures. Tetrahedron 2015, 71, 2463-2481.

(15) Park, T.-S.; Kim, H.-W. USES OF SESQUITERPENE DERIVATIVES. EP2331089 (A2), 2011.

(16) Murray, J. I.; Spivey, A. C. Amines vs. N -Oxides as Organocatalysts for Acylation, Sulfonylation and Silylation of Alcohols: 1-Methylimidazole N-Oxide as an Efficient Catalyst for Silylation of Tertiary Alcohols. Adv. Synth. Catal. 2015, 357, 3825-3830.

(17) Nishimoto, Y.; Okita, A.; Yasuda, M.; Baba, A. Synthesis of a Wide Range of Thioethers by Indium Triiodide Catalyzed Direct Coupling between Alkyl Acetates and Thiosilanes. Org. Lett. 2012, $14,1846-1849$.

(18) Álvarez-Calero, J. M.; Jorge, Z. D.; Massanet, G. M. $\mathrm{TiCl}_{4} / \mathrm{Et}_{3} \mathrm{~N}-\mathrm{Mediated}$ Condensation of Acetate and Formate Esters: Direct Access to $\beta$-Alkoxy- and $\beta$-Aryloxyacrylates. Org. Lett. 2016, 18, $6344-6347$.

(19) Dornhaus, F.; Scholz, S.; Sänger, I.; Bolte, M.; Wagner, M.; Lerner, H.-W. A Comparative Study on the Structural and Chemical Properties of Group 13-15 Element Addition Compounds t-Bu $2 \mathrm{PH} \cdot \mathrm{EX}_{3}$ $(\mathrm{E}=\mathrm{B}, \mathrm{Al}, \mathrm{Ga}, \mathrm{In} ; \mathrm{X}=\mathrm{Cl}, \mathrm{Br})$. Z. Anorg. Allg. Chem. 2009, 635, 2263-2272.

(20) Montgomery, T. P.; Grandner, J. M.; Houk, K. N.; Grubbs, R. H. Synthesis and Evaluation of Sterically Demanding Ruthenium Dithiolate Catalysts for Stereoretentive Olefin Metathesis. Organometallics 2017, 36, 3940-3953.

(21) Bruno, N. C.; Tudge, M. T.; Buchwald, S. L. Design and Preparation of New Palladium Precatalysts for C-C and C-N Cross-Coupling Reactions. Chem. Sci. 2013, 4, 916-920.

(22) Aranyos, A.; Old, D. W.; Kiyomori, A.; Wolfe, J. P.; Sadighi, J. P.; Buchwald, S. L. Novel ElectronRich Bulky Phosphine Ligands Facilitate the Palladium- Catalyzed Preparation of Diaryl Ethers. J. Am. Chem. Soc. 1999, 121, 4369-4378. 
(23) Grim, S. O.; McFarlane, W.; Davidoff, E. F. Group Contributions to Phosphorus-31 Chemical Shifts of Tertiary Phosphines. J. Org. Chem. 1967, 32, 781-784.

(24) Hesp, K. D.; Stradiotto, M. Stereo- and Regioselective Gold-Catalyzed Hydroamination of Internal Alkynes with Dialkylamines. J. Am. Chem. Soc. 2010, 132, 18026-18029.

(25) Rotta-Loria, N. L.; Chisholm, A. J.; MacQueen, P. M.; McDonald, R.; Ferguson, M. J.; Stradiotto, M. Exploring the Influence of Phosphine Ligation on the Gold-Catalyzed Hydrohydrazination of Terminal Alkynes at Room Temperature. Organometallics 2017, 36, 2470-2475. 